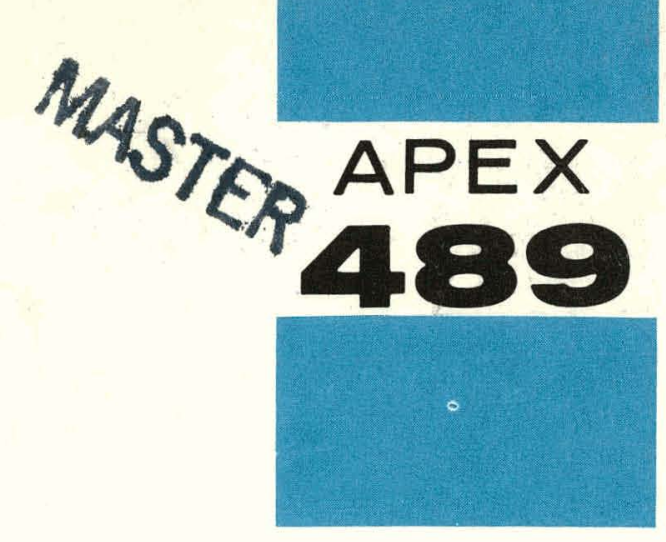

\title{
Space-Energy Cell Homogenization
}

\author{
B.H. Duane \\ M.J.Stanley
}

GENERAL G ATOMIC PRODUCTS DIVISION

REPRODUCIULE COPY 


\section{DISCLAIMER}

This report was prepared as an account of work sponsored by an agency of the United States Government. Neither the United States Government nor any agency Thereof, nor any of their employees, makes any warranty, express or implied, or assumes any legal liability or responsibility for the accuracy, completeness, or usefulness of any information, apparatus, product, or process disclosed, or represents that its use would not infringe privately owned rights. Reference herein to any specific commercial product, process, or service by trade name, trademark, manufacturer, or otherwise does not necessarily constitute or imply its endorsement, recommendation, or favoring by the United States Government or any agency thereof. The views and opinions of authors expressed herein do not necessarily state or reflect those of the United States Government or any agency thereof. 


\section{DISCLAIMER}

Portions of this document may be illegible in electronic image products. Images are produced from the best available original document. 


\section{LEGAL NOTICE}

This report was prepared as an account of Government sponsored work. Neither the United States, nor the Commission, nor any person acting on behalf of the Commission:

A. Makes any warranty or representation, expressed or implied, with respect to the accuracy, completeness, or usefulness of the information contained in this repnrt, or that the use of any information, apparatus, method, or process disclosed in this report may not infringe privately owned rights; or

B. Assumes any liabilities with respect to the use of, or for damages resulting from the use of any information, apparatus, method, or process disclosed in this report.

As used in the above, "person acting on behalf of the Commission" includes any employee or contractor of the Commission, or employee of such contractor, to the extent that such employee or contractor of the Commission, or employee of such contractor prepares, disseminates, or provides access to, any information pursuant to his employment or contract with the Commission, or his employment with such contractor.

Printed in USA. Price $\$ 3.00$. Available from the

Office of Technical Services

U. B. Departmont of Commerce

Washington 25, D. C. 


\title{
Space-Energy Cell Homogenization
}

\author{
B.H. Duane \\ M. J.Stanley
}

Nuclear Development Laboratories Sub-Section

August 1, 1958

United States Air Force

United States Atomic Energy Commission
Contract No. AF 33(600)-38062

Contract No. AT (11.1)-171

\author{
GENERAL ESTECTRIC \\ ATOMIC PKUUUCTS DIVISION \\ AIRCRAFT NUCLEAR PROPULSION DEPARTMENT \\ CINCINNATI 15, OHIO
}

Published by

Technicol Fublicutlons Sub-Section

July 1959 


\section{Abstract}

Fine-structure nuclear analysis program S (GE-ANPD APEX-394, "Neutron and Photon Transport: Plane, Cylinder, Sphere") has been extended to provide properly averaged nuclear data for grossstructure analysis work.

The input extension consists of the gross-structure space-energy lattice. Cell homogenization is performed by direct space-energy integration of each of the reaction-rate terms in the transport equation, with preservation of the detailed balance of leakage, collision, scatter-transfer, fission production, and fixed-source production. On a flexible flux-or-adjoint weighted space-energy cell basis, the extended output includes: cell-homogenized cross sections for collision, absorption, scatter, scatter-transfer, and fission multiplication; field depression or accentuation; leakage; surface albedo; and field diffusion coefficient, with inclusion of lumped-void leakage enhancement and other fine-structure heterogeneity effects.

Specific areas of immediate engineering applicability in GE-ANPD fuel loading and gross power shaping work on epithermal reactors include: (1) two-energy albedo input to program $K$ with inclusion of the effects of complex control rod cell structure and of energy degradation within a control rod cell, (2) complete three-energy input to program CURE and to program $F_{3}$ with inclusion of the effects of fine-structure void channels and strong absorbers and of three-energy control-rod cell albedos, and (3) nineteen-energy flux depression factors and control-rod cell albedos for input to program $G_{2}$. 


\section{Contents}

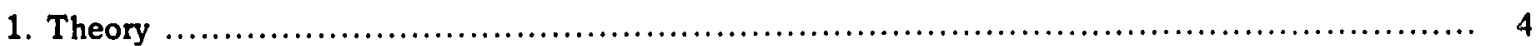

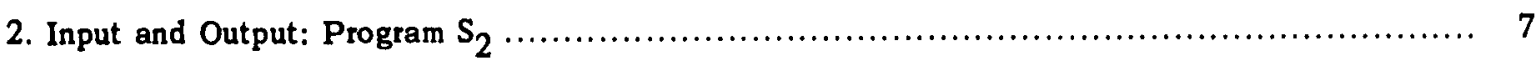

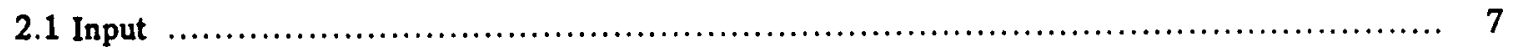

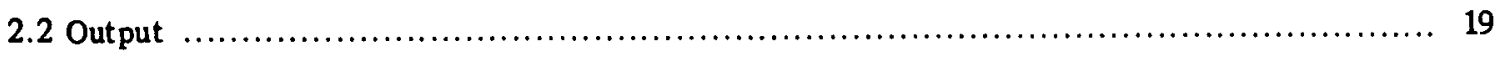

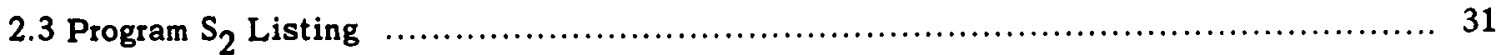

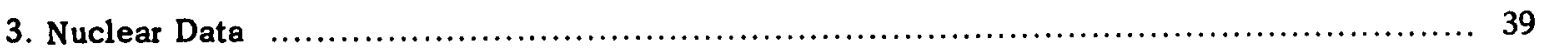

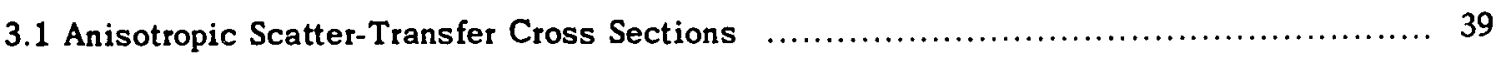

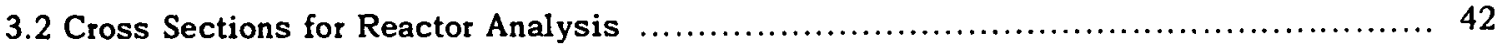




\section{Theory}

The neutron transport equation solved by the interim version of digital computer program $S$ has the canonical form

$$
\begin{aligned}
& \nabla \cdot \underline{\Omega} \phi(\underline{r}, p)+\Sigma(\underline{r}, p) \phi(\underline{r}, \underline{p}) \\
& =\int_{0}^{\infty} \phi\left(\underline{r}, p^{\prime}\right) \cdot(4 \Pi)^{-1} \Sigma_{s}\left(\underline{r}, p^{\prime} \mid p\right) p^{\prime 2} d p^{\prime} \\
& +(4 \pi k)^{-1} S(p) \int_{0}^{\infty} \dot{\phi}\left(\underline{r}, p^{\prime}\right) \nu \Sigma_{f}\left(\underline{r}, p^{\prime}\right) p^{\prime 2} d p^{\prime},
\end{aligned}
$$

where $\phi(\underline{r}, \underline{p}) d V(\underline{r}, \underline{p})$ is the neutron flux in position $(\underline{r})$ and momentum $(\underline{p})$ volume element $d V(\underline{r}, p)$, $\underline{\Omega}$ is the unit vector $\phi / p, \Sigma$ is the collision probability per path length, $\Sigma_{s}$ is the scatter-transfer probability per path length, $k$ is the reactivity eigenvalue, $S(p)$ is the fission production spectrum, and $\nu \Sigma_{f}$ is the fission multiplication probability per path length.*

The space-energy cell homogenization performed by the program $S_{2}$ extension proceeds by direct integration of each of the reaction rate terms in this balance equation, with preservation of the detailed balance of leakage, collision, scatter-transfer, and fission production within each spaceenergy cell. Multiplying equation (1.1) by a phase-space volume element $d_{I} V(r) p^{2} d_{N} p d \Omega$ and integrating over a space-energy cell $(I, N)$, with preservation of the detailed balance of reaction rates, leads to the homogenized representation for flux and reaction probabilities, ${ }^{\dagger}$

$$
\begin{aligned}
& V(I)=\int d_{I} V(\underline{r}), \\
& \phi(I, N)=\int \phi(\underline{r}, p) d_{I} V(\underline{r}) p^{2} d_{N} p / V(I), \\
& \Sigma(I, N)=\int \phi(\underline{r}, p) \Sigma(\underline{r}, p) d_{I} V(\underline{r}) p^{2} d_{N} p / \phi(I, N) V(I), \\
& \Sigma_{s}\left(I, N^{\prime} \mid N\right)=\int \phi\left(\underline{r}, p^{\prime}\right) \Sigma_{s}\left(\underline{r}, p^{\prime} \mid p\right) d_{I} V(\underline{r}) p^{\prime 2} d_{N^{\prime}} p^{\prime} p^{2} d_{N} p / \phi\left(I, N^{\prime}\right) V(I), \\
& S(N)=\int S(p) p^{2} d, p, \\
& \nu \Sigma_{f}(I, N)=\int \phi(\underline{r}, p) \nu \Sigma_{f}(\underline{r}, p) d_{I} V(\underline{r}) p^{2} d_{N} p / \phi(I, N) V(I) .
\end{aligned}
$$

*GE-ANPD APEX-394, "Neutron and Photon Transport: Flane, Cylinder, Sphere," by B. H. Duane, discusses validity limitations. Anisotropic scatter-transfer is neglected.

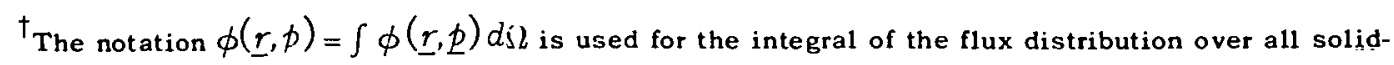
angle elements $d ! 2$. 
Similarly, the requirement that cell leakage be preserved leads uniquely to definition of the flux diffusion coefficient $D(I, N)$ for an homogenized cell by the relation*

$$
\int \nabla \cdot \underline{J}(\underline{r}, p) d_{I} V(\underline{r}) p^{2} d_{W} p=-\int \nabla \cdot D(I, N) \nabla_{\phi}(\underline{r}, p) d_{I} V(\underline{r}) p^{2} d_{N} p
$$

Performing the indicated integration by conversion to surface integrals yieids

$$
D(I, N)=-\int \underline{J}(\underline{r}, N) \cdot d_{I} \underline{A}(\underline{r}) / \int \nabla_{\phi}(\underline{r}, N) \cdot d_{I} \underline{A}(\underline{r})
$$

where $D(I, N)$ is the leakage-preserving flux diffusion coefficient for the homogenized cell, $I(r, N)=$ $\int \underline{J}(\underline{r}, p) p^{2} d_{N} p$ is energy-band integrated current, $\phi(r, N)=\int \phi(r, p) p^{2} d_{N} p$ is energy-band integrated flux, and $d_{I} A(\underline{r})$ is an element of the surface area enclosing the cell. It is to be noted that this argument has both necessary and sufficient character. There is no other diffusion coefficient that preserves cell leakage.

For a reactor having slab, cylindrical, or spherical symmetry ('vith arbitrarily complex heterogeneity, which may include void gaps, annuli, or shells), equation (1.8) simplifies to

$$
D(I, N)=-\frac{A\left(I_{+}\right) J\left(I_{+}, N\right)-A(I-.) J(I-, N)}{A\left(I_{+}\right) \nabla_{\phi}\left(I_{+}, N\right)-A(I-) \nabla_{\phi}(I-, N)},
$$

where $I \pm$ refers to inner and outer surfaces bound ing cell $I, J$ is current, and $\nabla \phi$ is flux gradient evaluated on the interior side of the cell surface area $A$.

Expressed in terms of these cell-homogenized quantities, the isotropic component of the neutron transport equation (1.1) becomes

$$
\begin{gathered}
-D(I, N) \int \nabla_{\phi}(\underline{r}, N) \cdot d_{I^{-}} \underline{\underline{-}}(\underline{r})+\Sigma(I, N) \phi(I, N) V(I) \\
=\sum_{N^{\prime}} \phi\left(I, N^{\prime}\right) \Sigma_{S}\left(I, N^{\prime} \mid N\right) V(I) \\
+k^{-1} S_{N} \sum_{N^{\prime}} \phi\left(I, N^{\prime}\right){ }_{\nu \Sigma_{f}}\left(I, N^{\prime}\right) V(I) .
\end{gathered}
$$

A transition to the gross-structure multienergy neutron diffusion equation now can be formulated on a secure logical basis by summing the last equation over an array of space cells, dividing by the volume of the array, replacing the cell label $I$ by a gross position vector $\underline{R}$, and making use of the surface integral definition for the gross divergence operator ${ }^{\dagger}$ to obtain

$$
\begin{aligned}
-\nabla & \cdot D(\underline{R}, N) \nabla_{\phi}(\underline{R}, N)+\Sigma(\underline{R}, N) \phi(\underline{R}, N) \\
& =\sum_{N^{\prime}} \phi\left(\underline{R}, N^{\prime}\right) \Sigma_{s}\left(\underline{R}, N^{\prime} \mid N\right) \\
& +k^{-1} S_{N} \sum_{N^{\prime}} \phi\left(\underline{R}, N^{\prime}\right) \nu \Sigma_{f}\left(\underline{R}, N^{\prime}\right) .
\end{aligned}
$$

*The notation $\underline{J}(\underline{r}, p)=\int \underline{\Omega} \psi(\underline{r}, \underline{p}) d \Omega$ is used for the integral of the current distribution over all solid angle elements $d \Omega$.

$$
{ }^{\dagger} \nabla \cdot \underline{F}=\underset{V \rightarrow 0}{\operatorname{Limu}}\left[\int \underline{F} \cdot d \underline{A} / \int d V\right]
$$


Constructing the diffusion coefficient on a cell-leakage-preserving basis, in accordance with equation (1.8), provides maximum assurance of retaining a useful level of reliability, on at least a cellaverage basis, even when engineering practicalities necessitate pushing diffusion theory considerably beyond its normal domain of applicability. 


\section{Input and Output: Program $S_{2}$}

\subsection{INPUT}

An initial checkout study selected to delineate the limitations of the numerical analysis method in both voids and strong absorbers is shown in Figure 1. The input data following Figure 1 have been normalized to a void outer radius of unity to provide dimensional invariance in interpretation of the output. The input deck assembly is given in Table 1.

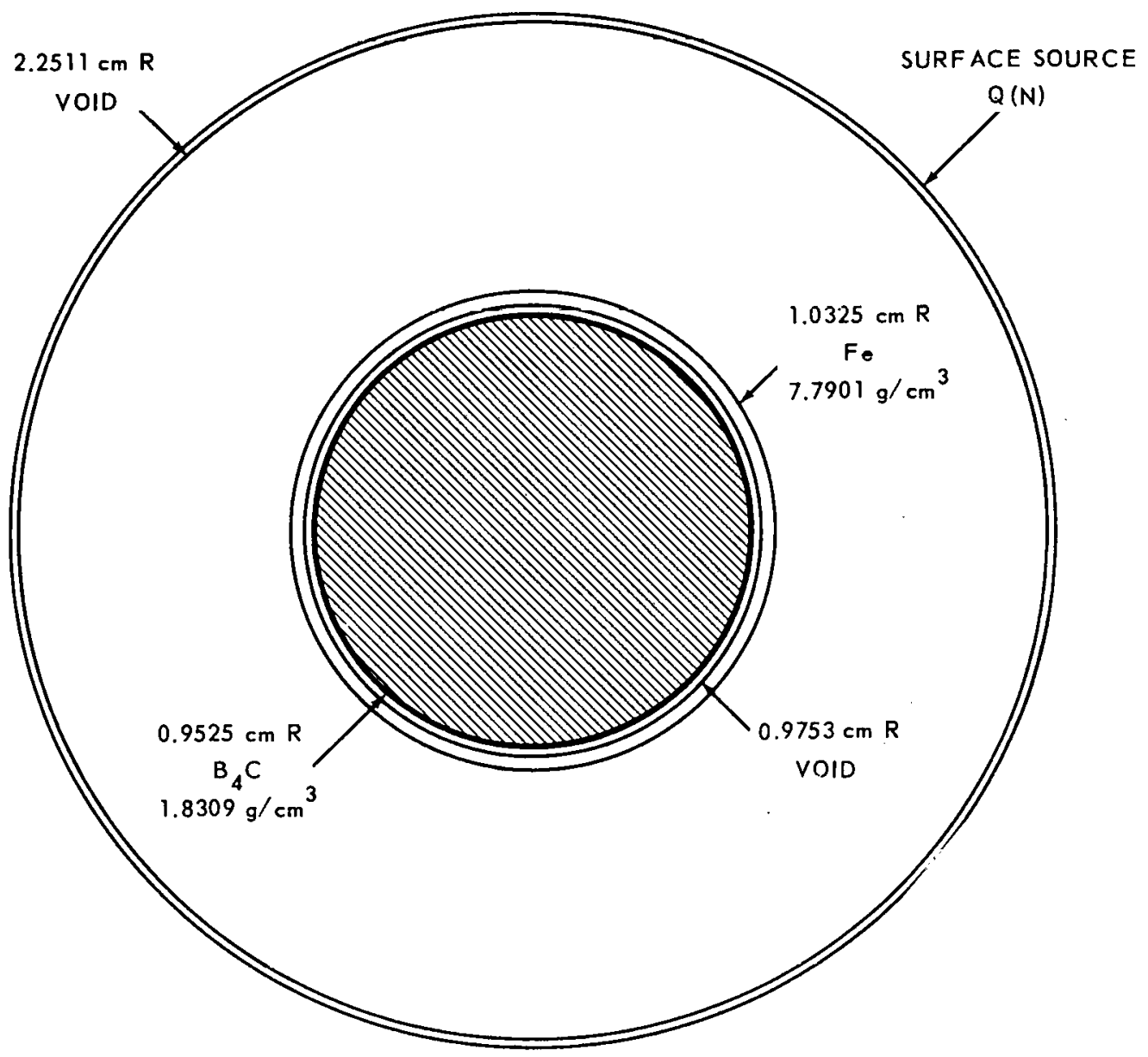

Fig. 1-Control rod in void annulus 
$5 I 500000$ d +0000100 d d $1+0000000$ d 00000000 d d 00000000 d 100000000 d 00000000 dd 000000000 d p 800

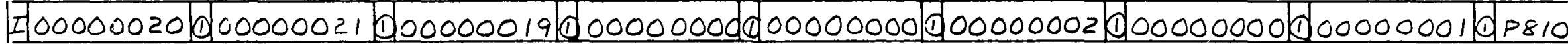

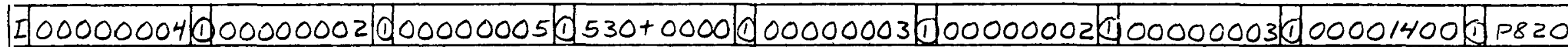
d 4040076764 0048271401 (44) 11
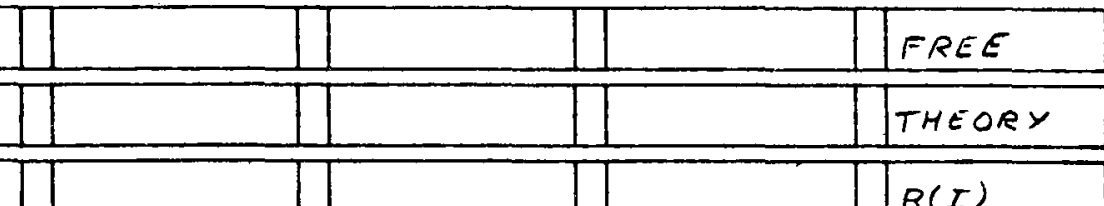

0014811050164

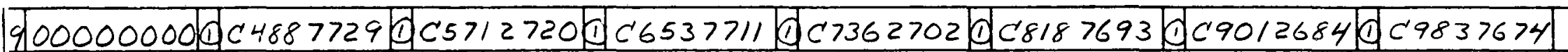

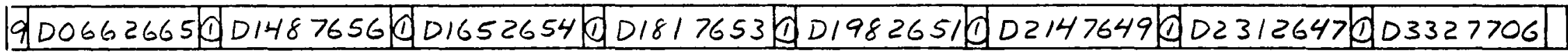

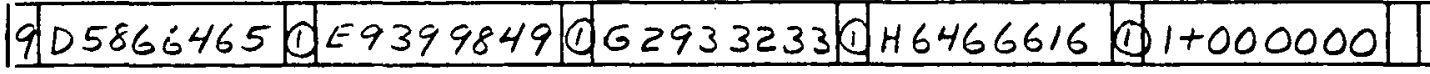

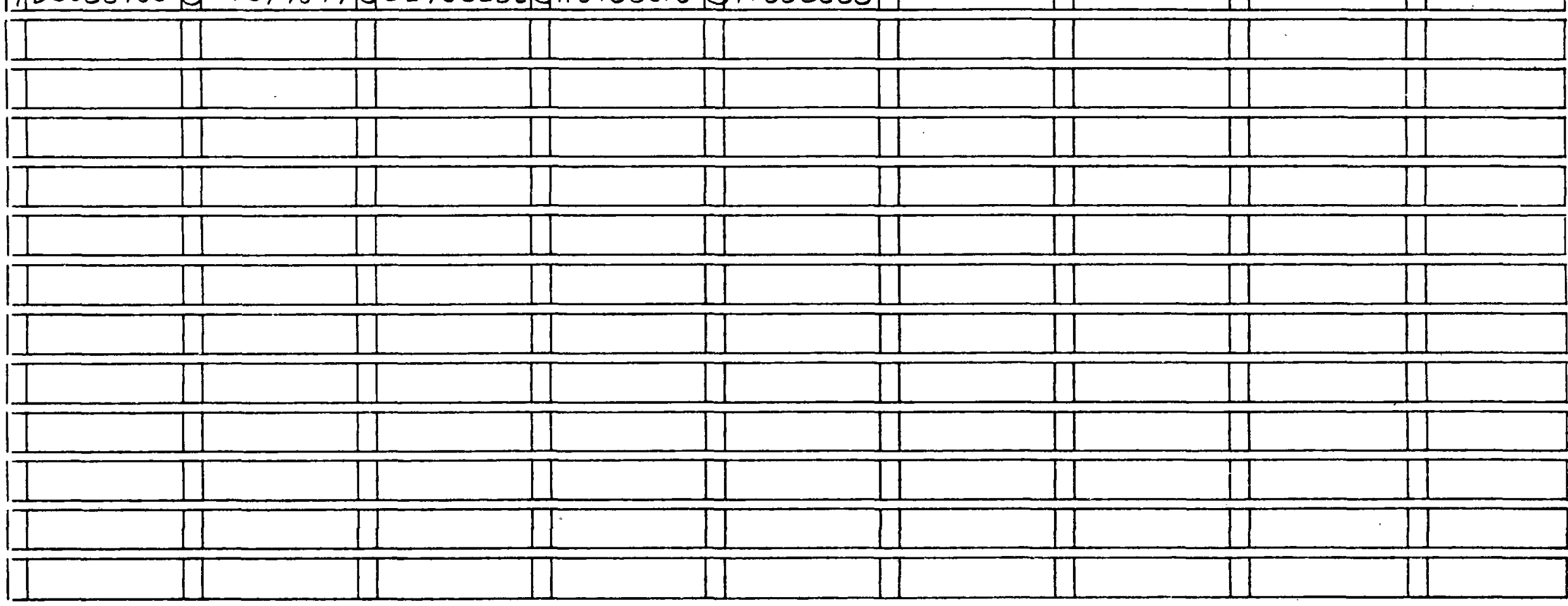


Q1-ANP Reactor Analrolo Program S leutros or Pboton Transport

Slab, Cylinder, or Sphere

PROBABILITY/PATH

$(/ C M)$

$\mathrm{B}_{4} \mathrm{C} \quad 2.519 \mathrm{G} / \mathrm{CC}$

Phys1ca, Inpur, Outout: GE-ANPD APEX-394

Watheat 1C8: IA-1891

Program: T-1-140, T-1-130

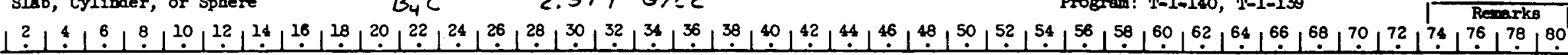
48.2053004010053055

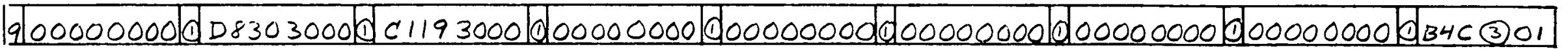

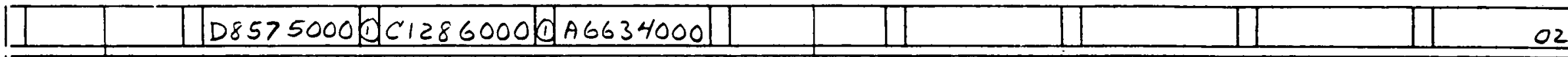

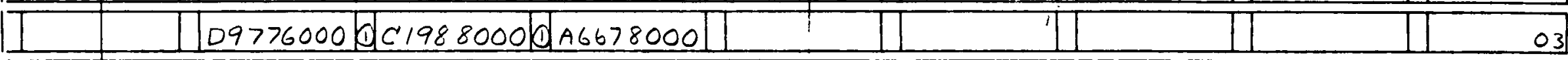

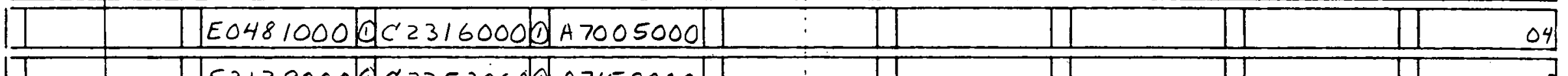

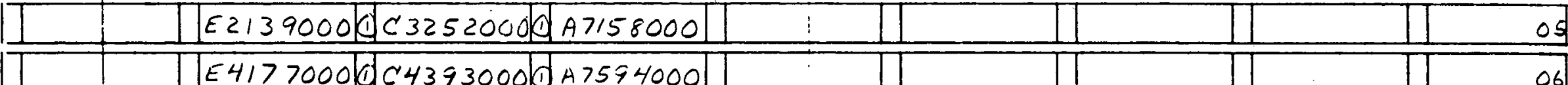

$c$
$z$
5
5
5
in
on
-1
4
0
0

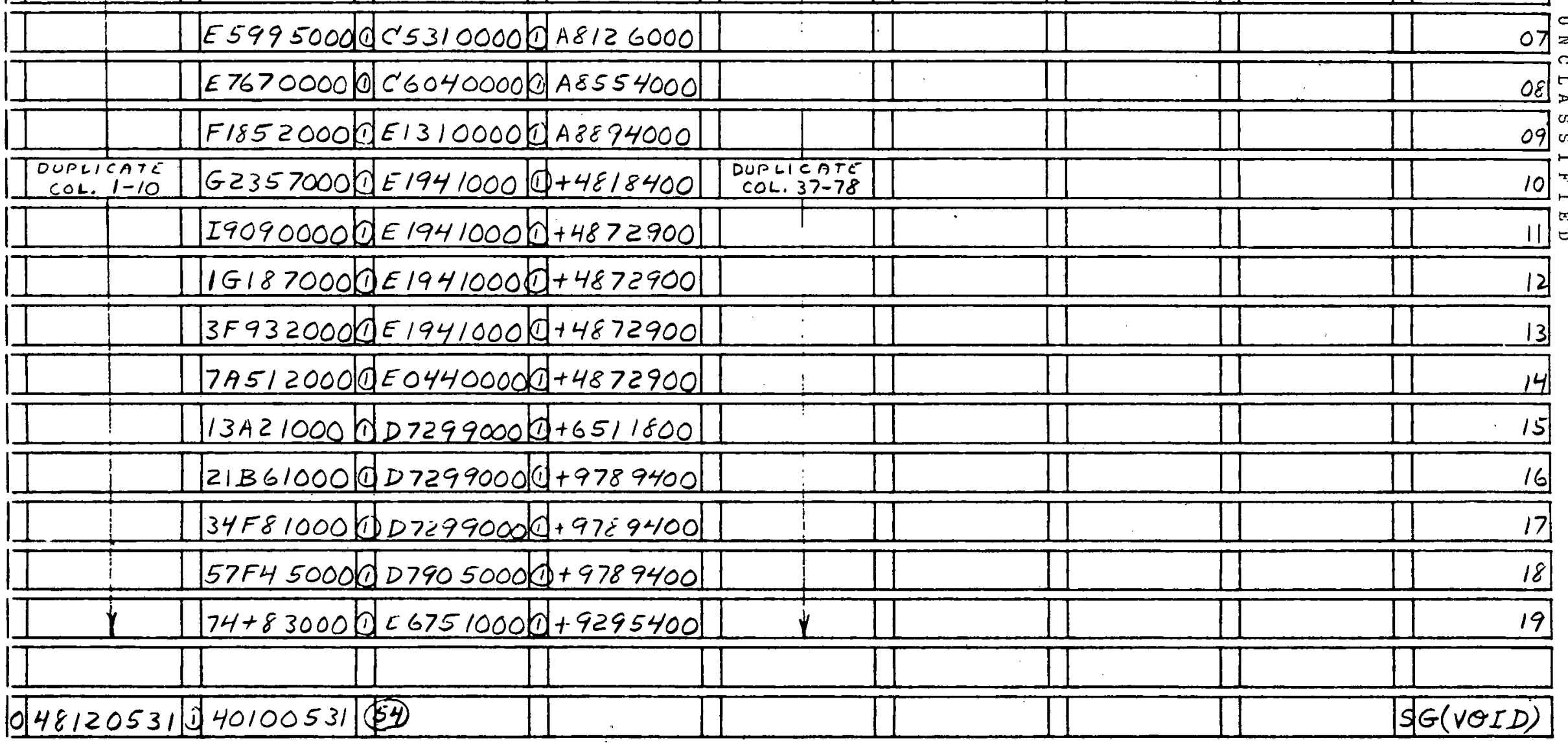


GS-AIP Resct lalyois Program S Beutron or Photon Transport Slab, Cylinder, or Sphere

PROBABILITY/PATH $(/ C M)$

Physic8, Inpur, vutput: GE-ANPD APEX-394

Mthematics: IA-1891

Program: T-1-140, T-1-139

FE $\quad 7.87 \mathrm{G} / \mathrm{CC}$

b48120532040100532 (55)

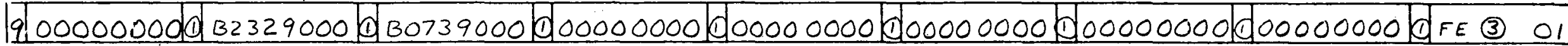

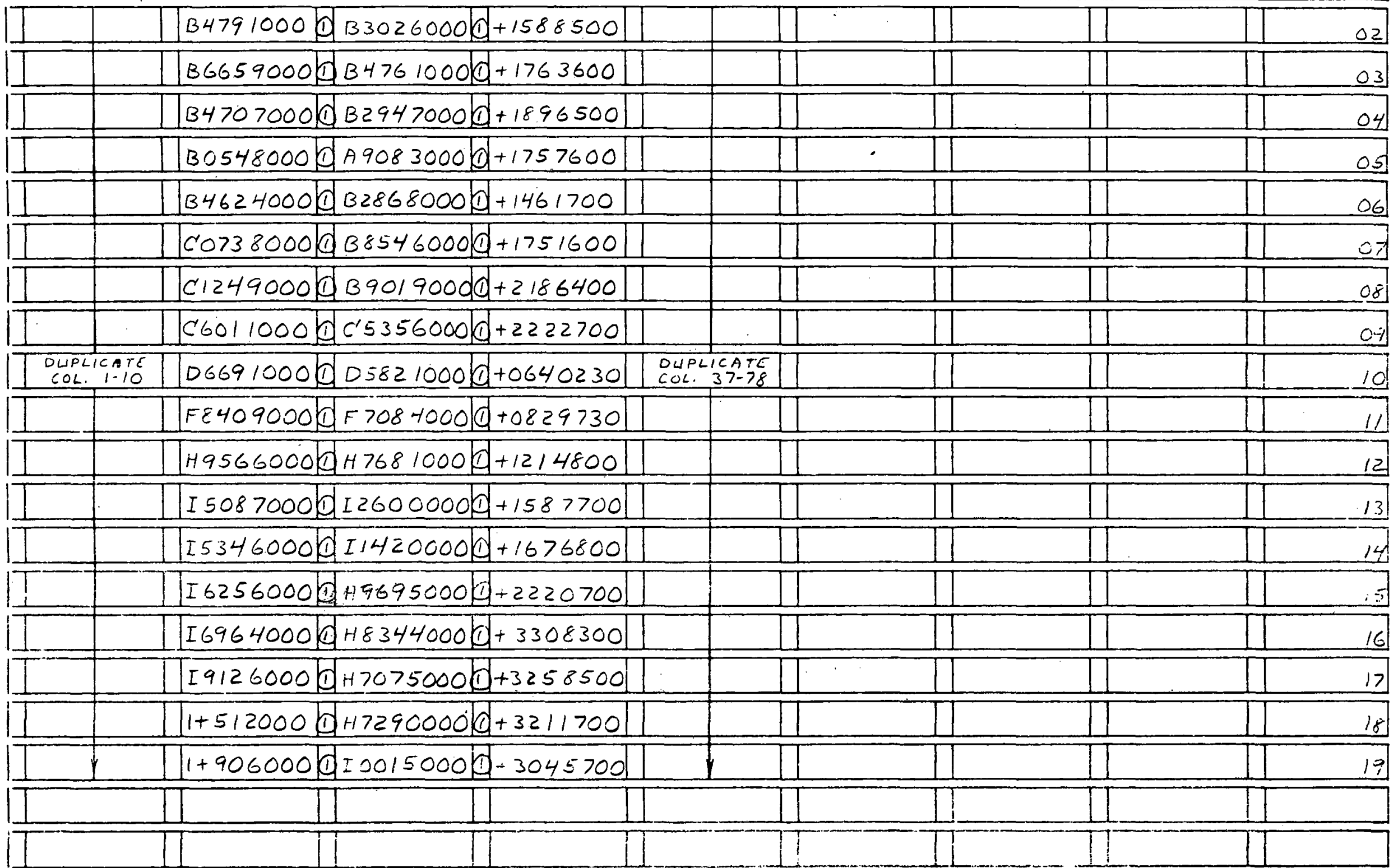


GR-AlP Reactor Apalysis Program S

Reutron or Photon Transport

Slab, Cylinder, or Sphere

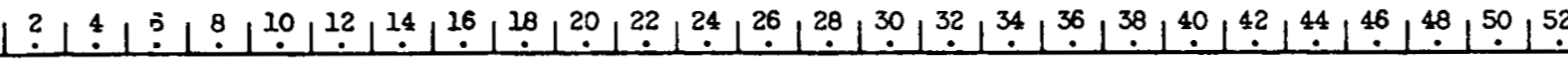

Phys1cs, Input, Output: GE-ANPD APEX-394

Mathemat1cs: IA-1891

Program: T-1-140, T-1-130 008100502 (64) II 1 $\operatorname{MAT}(z)$

000000530000000530000000530 d0 000005300000005300000005300000005300000005300

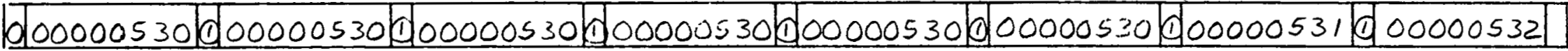
000000531000000531000000531000000531] $0.48100504(64)$ 11 $\prod$ II

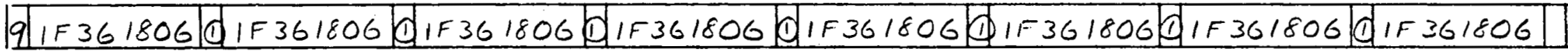

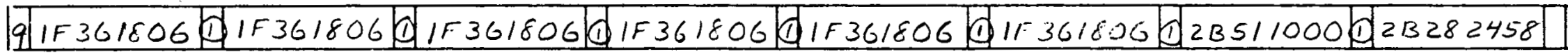
g]2B51100002B511000 23511000023511000

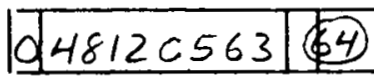

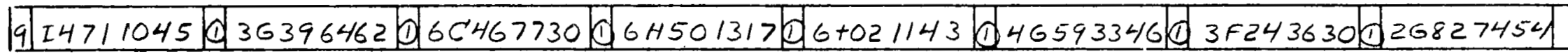

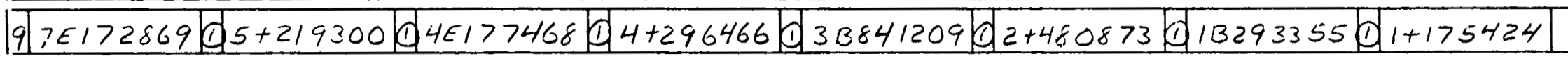

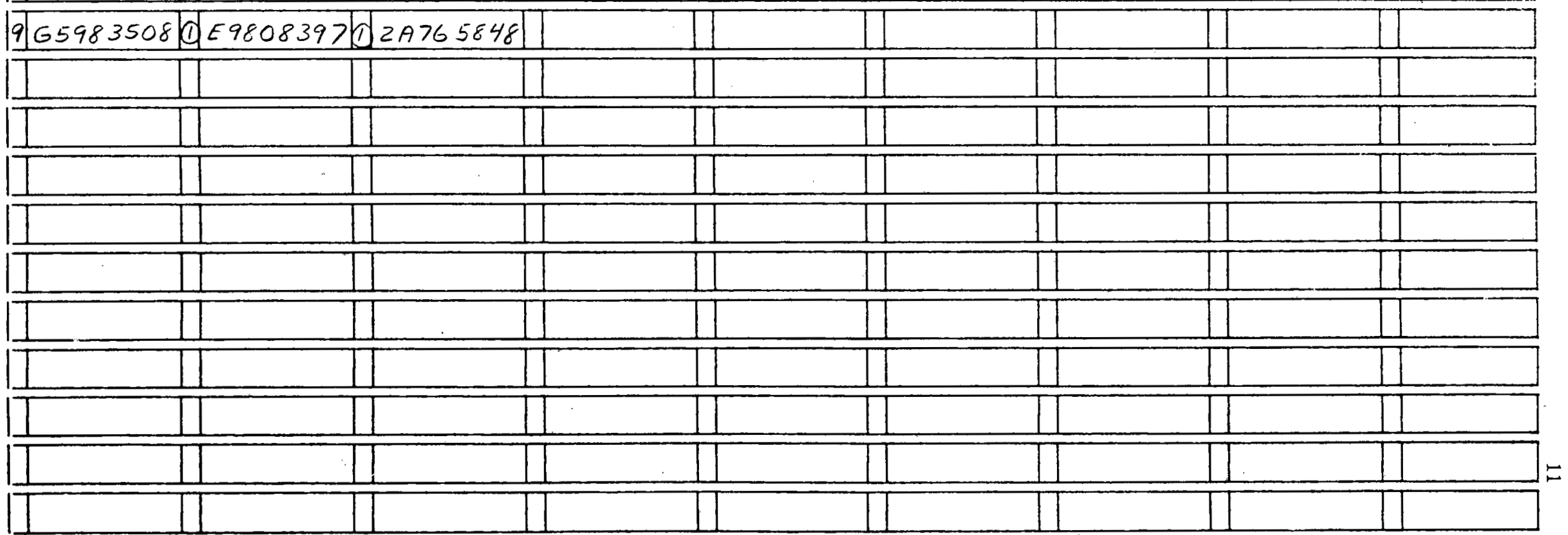


Ge-AIP Pe : Amalyisis Program S Feutron or Fhoton Tranoport Slab, Cylinder, or Sphere

SENSORS IN $7.31 \mathrm{G} / \mathrm{CC}$

CU 8.94 G/CC
Physice, Input, Output: GE-ANFO AFEX-39

Watheratics: IA-1891

Program: T-1-140, T-1-139

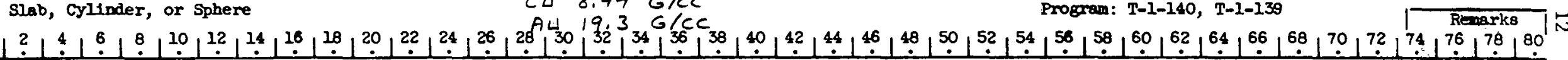
|01482405700148120570(⿻5

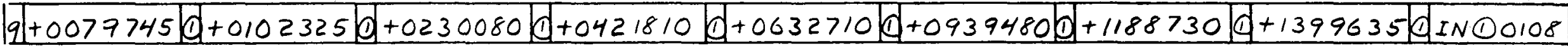

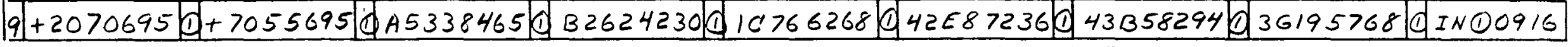
\begin{tabular}{|l|l|l|l|l|l|l|}
\hline 93278460 d $4 E 757214$ d $6 E 978389$ (46) & & & & & & INO1719 \\
\hline
\end{tabular} $9+0001795 d+0002305 d+0002955 d+0003805 d+0004880 d+0006255 d+00080400+001032000400108$

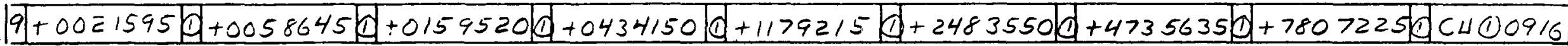

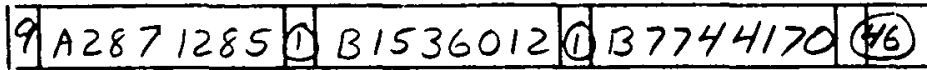

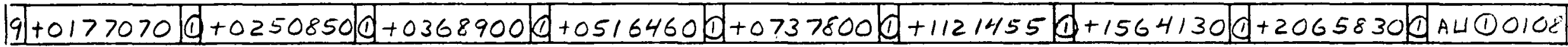

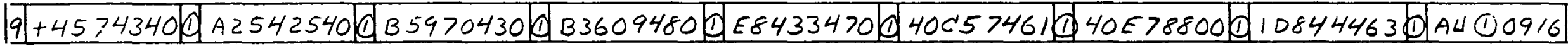
19]2B8126630 3632772600625736960

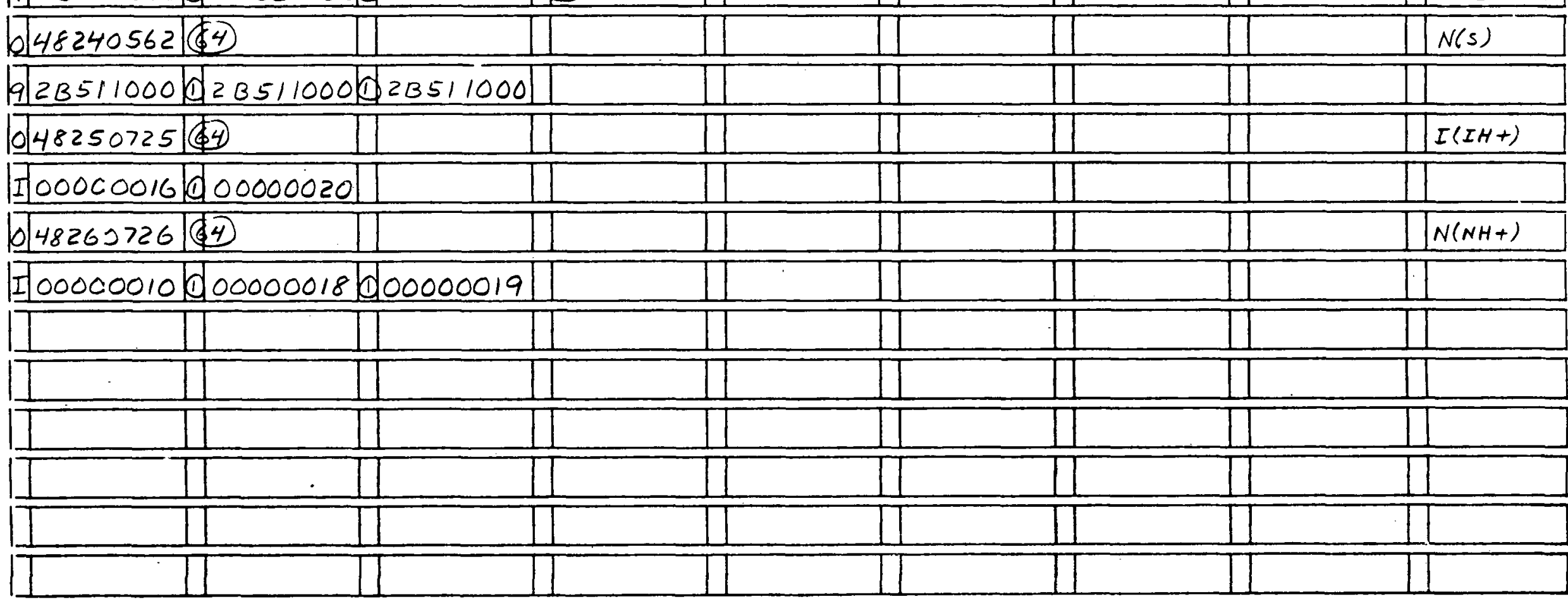


CB-AIP Reactor ARalsBis Program S

Neutron or Photon Transport

CYLINDER SH CONSTANTS

PhysicB, Input, Output: GE-AMFD AFEX-394

slab, Cylinder, or sphere

thematicB: LA-1891

Progran: T-1-140, T-1-130

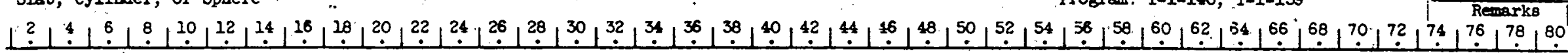

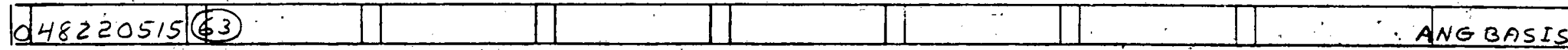

95.1870515056787443000000000 da 6787443 d A 1870515 |0.48220516

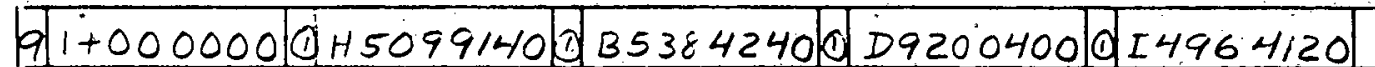
| 48220517 !

900000000 i 14964120009200400 d B 5384240 d H 5099140 b. $4822052 d$.

$900000000010 I 496412012392636>012 B 92636>0010196412$ d48210521

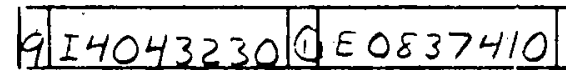
bا48210522 A $F .52145 .20$ (a) $C 478548 d$ II

b] $48100503(64$ 14810051464 b. 48120523 (48 $400523(55)$ II.

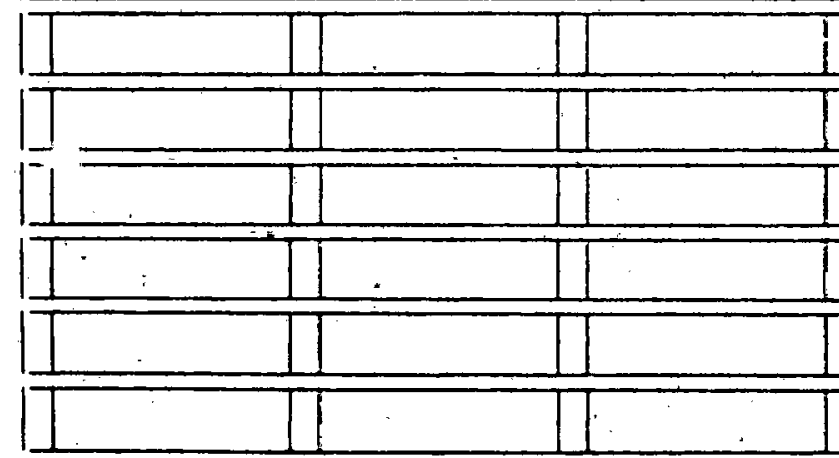
11 11 11 : !:

I!

$1 ! \div \quad !$

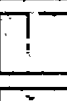

11

11

\begin{tabular}{|l|}
11 \\
$\| 1$ \\
\hline 1
\end{tabular}




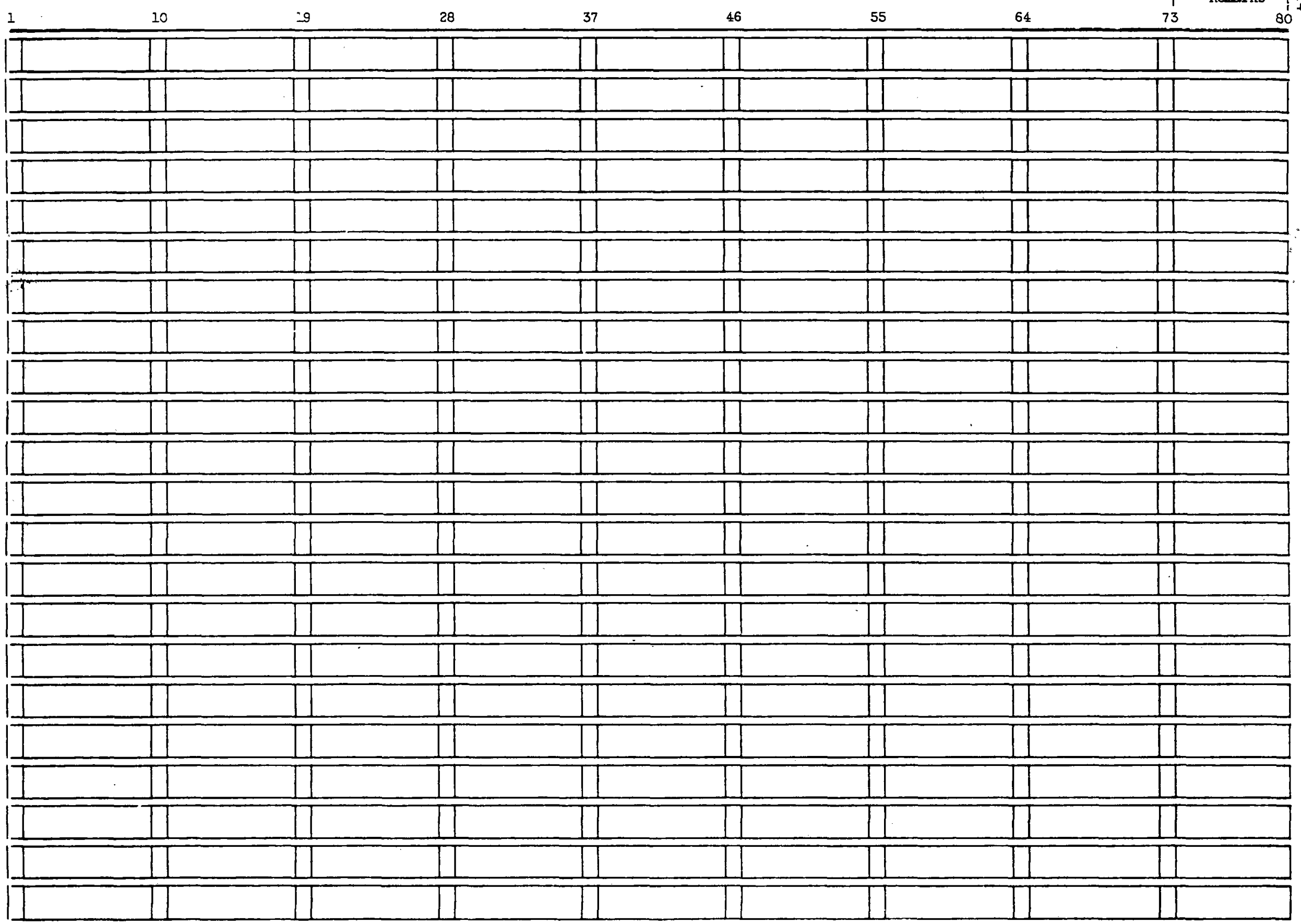


TABLE 1

INPUT DECK ASSEMBLY: PROGRAM S 2

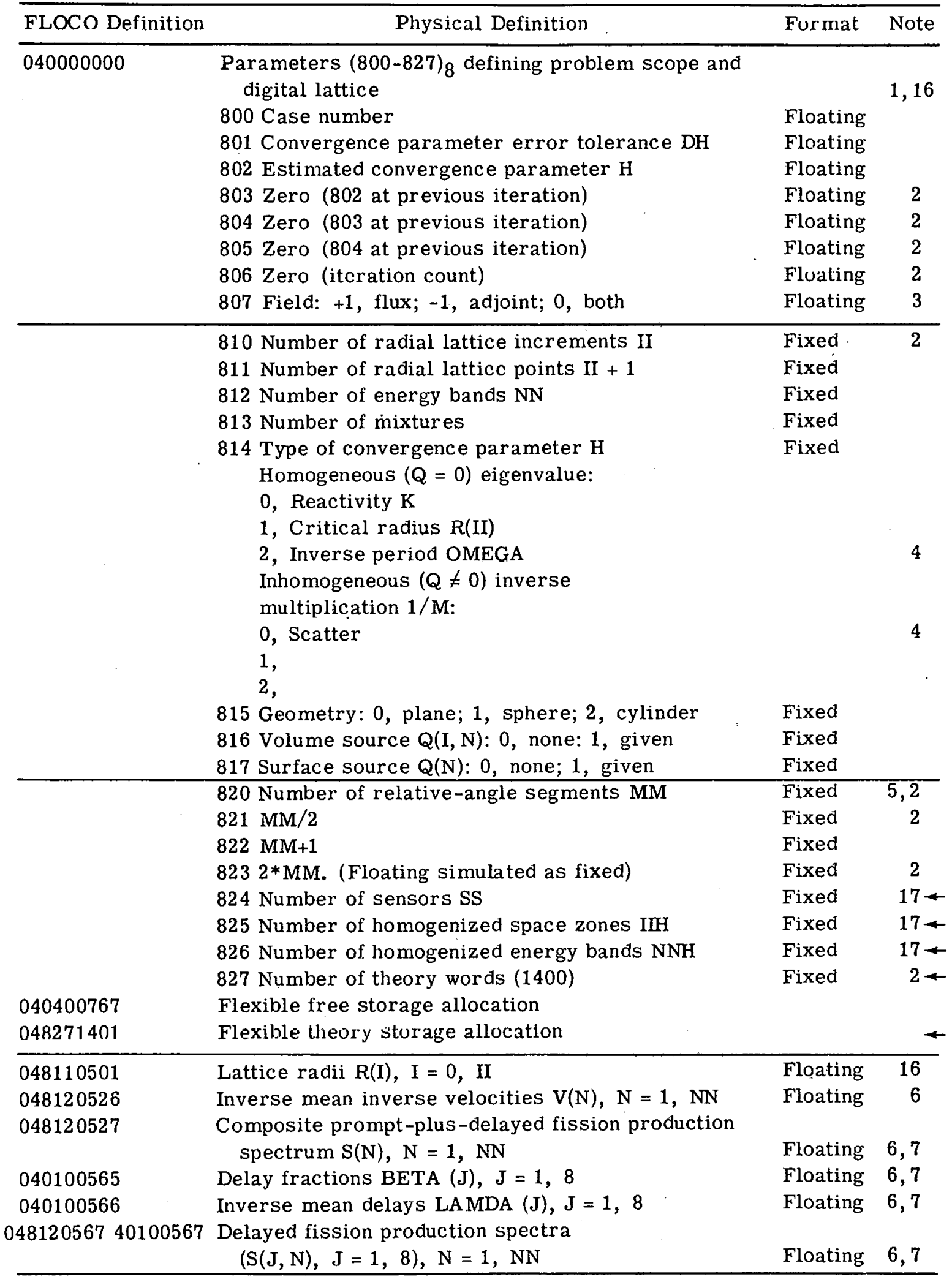


TABLE 1 (Cont。)

INPUT DECK ASSEMBLY: PROGRAM $\mathrm{S}_{2}$

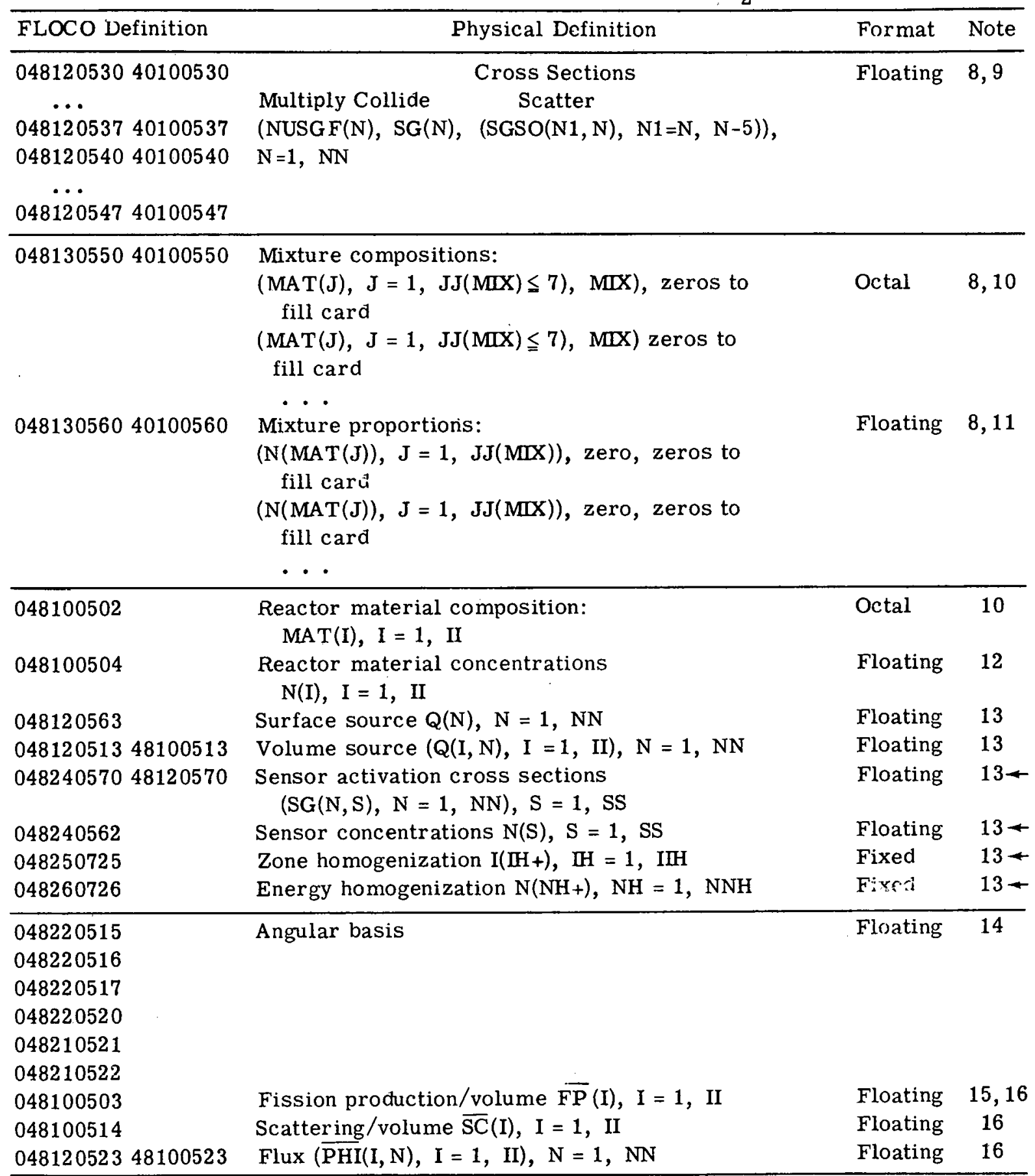




\section{TABLE 1}

\section{INPUT DECK ASSEMBLY: PROGRAM $\mathrm{S}_{2}$}

\section{NOTES}

1. Parameter block is to be recoded so as to eliminate forward reference in physical definitions.

2. Entry is to be stored in program or computed by program rather than fed in as input, at next recoding.

3. Entry is to be shifted to fixed point card at next recoding.

4. Operating in a useful form, but incomplete.

5. Number of cylindrical-geometry polar-angle lattice points $L L$ is equal to number of relativeangle segments $M M$.

6. Control card may be omitted in steady-state system.

7. Control card may be omitted if system contains no fissile material. Normalization:

$$
\sum_{N} S(N)=1=\sum_{N} S(J, N) .
$$

8. Flexibility:

$$
N(I) \sum_{M A T(I)} N(M A T) S G(M A T)=\text { PROBABILITY } / \text { PATH }
$$

Materials may be defined either directly, by entry of cross section storage allocation and data, or indirectly, by entry of storage allocation and specifications for mixing up to 7 previously defined materials or previously defined mixtures. Control cards not needed may be omitted.

9. For endothermic scatter, $N=1$ labels high energy flux or low energy adjoint. For exothermic scatter, $N=1$ labels low energy flux or high energy adjoint. If adjoint of stated problem is requested, machine transposes all transfer cross sections and inverts energy scale; user of program provides adjoint angle reversal by negative labelling of output current and leakage.

10. A material or mixture is entered as the last 3 octal integers of its FLOCO definition.

11. Machine computes $S G(M I X)=\sum_{J=1}^{J J(M I X)} N(\operatorname{MAT}(J)) S G(M A T)$,

for each successive mixture, and places these cross sections in the storage allocation reserved by previous entry of a FLOCO definition for the mixture.

12. Void regions, with $N(I)=0$, are admissible. Strong absorbers cause no trouble. The mirrorreflection boundary condition commonly used in infinite-lattice idealization is obtainable by enveloping a cell with a synthetic scattering film ten or so mean free paths thick $(\operatorname{SGSO}(N, N)=1$ $=S G(N)$, all other $S G=0)$. However, a more realistic cell-fine-structure analysis, with inclusion of leakage effects, is obtainable by working with a typical cell centered in an homogenized core.

13. Control card not relevant to the problem may be omitted.

14. Given in Table 2 of APEX-394. 
15. Initial estimate may be entered as 1 in fuel, 0 elsewhere.

16. Binary restart cards containing $\mathrm{P} 800-807, R(I), \overline{F P}(I), \overline{S C}(I), \overline{P H I}(I, N)$ from same problem, or from problem having the same $(I, N)$ lattice, may be inserted after these control cards to provide initial guess. Control card followed by no data sets a block to zero initial values.

17. Zero entry bypasses relevant subroutine. 
Changes from the previous formulation of the program have been marked by margin arrows. In Table 1, the parameters $824-827$ have been rearranged to make room for specification of the homogenized space-energy lattice dimensionality $(I I H, N N H)$. The number of theory words has been extended to 1400 to make room for equations (1.2-1.9). Control cards 401 (theory storage), 570 (sensor cross sections), and 562 (sensor concentrations) have been altered in correspondence with parameter block changes. Two new control cards $(725,726)$ have been inserted near the end of the input deck for entry of the space-homogenization lattice $I(I H+), I H=1, I I H$ and the energy-homogenization lattice $N(N H+), N H=1, N N H$.

In the illustration shown, the $(2,3)$ entries in 825 and 826 , respectively, call for a 2-zone, 3-energy homogenization. The entries $(16,20)$ and $(10,18,19)$ shown following the 725 and 726 control words, respectively, specify that homogenized zone $I H=1$ spans fine zones $I=1$ through $I=16$, that homogenized zone $I H=2$ spans fine zones $I=17$ through $I=20$, that homogenized energy band $N H=1$ spans fine energy bands $N=1$ through $N=10$, that homogenized energy band $N H=2$ spans fine energy bands $N=11$ through $N=18$, and that homogenized energy band $N H=3$ spans fine energy bands $N=19$ through $N=19$.

\subsection{OUTPUT}

Machine outout follows for the study shown in Figure 1. The flux-weighted space-energy homogenization blocks in the output list fixed source $Q(I H, N H)$, fission multiplication probability/path $N U S G F(I H, N H)$, collision probability/path $S G(I H, N H)$, scatter-transfer probabilities/path $S G S O(I H, N H-\wedge N H \mid N H)$, zoneaverage flux $P H I(I H, N H)$, leakage probability/path $L(I H, N H) /[V(I H) \cdot P H I(I H, N H)]$, flux diffusion coefficient $D(I H, N H)$, outer surface albedo $A(I H, N H)$, scatter probability/path $S G S O(I H, N H)$, and absorption probability/path $S G A(I H, N H)$.

A block of such information appears for each successive space-zone, followed by a similar block for the composite array of space-zones. (The study shown illustrates 2-zone, 3-energy output, with zone outer boundaries taken at the outer surface of the iron guide tube in Figure 1 and at the cell outer boundary, and with the energy divisions selected to span the fission production band, epithermal band, and thermal band.)

The output blocks of reaction probability/path and volume-averaged flux for the composite array of space-zones provide a compact summary of the nuclear performance of the cell. The leakage probability/ path for the composite array of zones can be employed directly as an effective transverse leakage "cross section" in matched radial-and-axial studies. Multienergy flux depression and accentuation factors, properly normalized to unity cell-average, are obtainable by dividing zone-average flux by cell-average flux for each energy band.

Control rod cells of arbitrary structural complexity can be entered into a gross-structure study either by use of the cell-albedo output or by use of the cell-homogenized reaction probability/path output. The latter method has the intrinsic advantage of portraying energy-degradation effects in greater detail.

The reaction probability/path blocks also are provided as binary-card output. This punched-card output can be fed back into the program as cross section input for a gross-structure study, on a selective basis, simply by replacing corresponding decimal cards in the input deck with these binary cards.

If adjoint output has been specified, an analogous adjoint-weighted space-energy homogenization is provided. It is to be noted that the labelling of the adjoint energy scale is inverted and that a negative sign is attached to adjoint current and leakage labels. 
PARAMETERS

$0.64000 \quad B B$ 5.95000000 0.000024 ud 0.00000400

$0.64000 \mathrm{BB}$ 0.00000000 0.00264100 $0.004740 \quad 00$ 0.00352100 0.00000000 0.00450200 0.60000 STZ 0.00000000 0.00736400 0.00635100 0.00515000 0.60000 STZ 0.000000 00 0.00000000 0.00622400 0.00766100 0.01141700 0.00000000 0.01152400 0.60000 STZ 0.01163600 0.60000 STZ 0.000000 0 . 00000 ST 0.60000 STZ 0.00000000 0.00100000

$0.64000 \quad B B$ 0.00000000 4.066266-01

4.586646-01
0.02250 $1.000000-06$ 0.00002500 0.00000200

0.01000

0.00231000 0.00266000 $0.0033150 \mathrm{C}$ 0.00365300 0.00000000 0.00462500 0.00002 0.00512500 0.00741000 0.00635300 0.00542300 0.00002 0.00000000 0.00000000 0.01052300 0.00000000 $0.0000000 C$ 0.00000000 0.00004

0.01250000 0.00001 0.00000000 0.00003

0.00000000

0.00201700

0.05125501 $3.488773-01$ 4.148765-01 5. $939985-0.1$
1.00000000 0.00002300 0.00000500

0.00235300 0.00274000 0.00000000 0.00366600 0.00000000 0.00000000

0.00615200 0.00743500 0.00635500 0.00567600

0.00632000 0.00000000 0.01136700 0.00000000 0.00000000 0.00000000

0.01334400

0.00000000

0.00000000 0.00150400

$3 \cdot 571272-01$ 4.165265-01

7.293323-01
0.00000000 0.00000000 8.00000000 0.00000000 $0.00000300<-0.00000200<-$

INDEX

0.00240200

0.00000000

0.003725

272500

0.00000000

$0.0064 \mathrm{Cl} \mathrm{CO}$ 0.00000000 0.00645000 0.00000000

0.00622100 0.00000000 0.01141400 0.01152100 0.01155700 0.00000000

$\mathrm{CORE}$
0.01337100

UTILIZATION

0.00000000

$0.001330 \quad 00$ 0.00243100 0.00337200 0.00412400 0.00440000 $0.003000 \quad 00$

0.00617600 0.00642500 0.00744200 0.00000000

0.00000000 0.00000000 0.00000000 0.00000000 0.00000000 0.00000000

0.00000000 0.00000000 0.00000000 0.00207200 0.00321000 0.00344500 0.00420300 0.00444200 0.00000000

0.00727000 0.00633200 0.00763500 0.00000000

0.00000300 0.00000000 $0.0000 \mathrm{cos} 00$ 0.00000 .00 0.01156200 0.01156300

0.00000000

0.00632200

0.00000000 $0.017400 \quad 00$

LATTICE RADII $R(I), I=0$, II $3.653771-01 \quad 3.736270-01$ $4.181765-01 \quad 4.198265-01$

$3.818769-01$ $4.214765-01$
Arrows

$0.00000000 \quad 0.00000000$ Changes

$0.00000300<-0.00257000<$

0.00252100

0.003174 OC

0.0000000

0.000000 00

0.00000000

0.00000000

0.00633700

$0.000000 \mathrm{CO}$

$0.000000 \quad 00$

$0.000000 \quad 00$ $0.000000 \quad 00$ 0.00000000 0.00000000 3.00000000 0.01161000

0.00000000

0.00632500

0.00000000 0.00000000

0.007340 .00 0.00634400 0.00000000 0.00000000

0.00000000 $0.00000 \mathrm{C} \quad 00$ 0.00000000 0.000000 .00 $0.00000 C$ OO 0.01101300

$0.00000 \mathrm{C} 00$ 0.00000000 0.00231000 $0.000000 \quad 00$

3.901268-01 3.983767-01 $4.231264-01 \quad 4.33277 \mathrm{C}-01$

$4.830300-01$

$4 \cdot 857500-01$

$40977600-01$

5.048100-01

5.213900-01

$5.417700-01$

5.599500-01

$5.767000-01$

$7.235700-0$

0.00000000

9.909000-01

$\operatorname{SGSO}(x, i n)$

$3 \cdot 11 y+00-0$ : $3 \cdot 1<8=00-C_{2}$ $3.198800-01$ $3.231600-\mathrm{C}$ $3 .: 13250=2$ $3.439300-0$ $3.531600-C$ $3.6,4600-0$ 131000-0

$5.1+50-0$

$5.194100-01$
CROSS SECTIONS

SGSO $(\mathrm{IN}-1, N)$
0.00000000
$1.663400-01$
$1.667800-01$
$1.700500-01$
$1.715800-01$
$1.759400-01$
$1.812600-01$
$1.855400-01$
$1.889400-01$
$4.818400-02$
$4.872900-02$

SCATTER

0.00000000 0.00000000 0.00000000 0.00000000 0.00000000 0.00000000 0.00000000 0.00000000 0.00000000
$\operatorname{SGSO}(N-4, N)$

0.00000000 $0.000000 \quad 00$ 0.00000000 0.00000000 0.00000000 0.00000000 0.00000000 $0.000000 \quad 00$ $c .00000000$ 0.00000000 0.00000000 
$0.000000 \quad 00$ $0.000000 \quad 00$ 0.00000000 0.00000000 0.00000000 0.00000000

0.64000 $0.60000 \mathrm{STZ}$ NUSGF(N) 0.00000000 0.00000000 C.000000 00 C. .00000000 C. 00000000 C. .00000000 .00000000 .00000000 C. 00000000 c. .00000000 c. .00000000 i. 00000000 0.00000000 0.00000000 . 0.00000000 0.00000000 0.00000000 0.00000000

1.71870000 3.69320000 7.15120000 1.3121000 : 2.1261000 3.4681000 5.76450001 7.40830001

5.194100-01

0.05423531 VOID 0.00023 $0.05676532 \mathrm{FE}$ 2.232900-01 2.479100-0 2.665900-0 2.470700-01 2.054800-01 $2.462400-01$ $3.073800-0$ 3.124900-0 3.601100-01 4.669100-0 $6.840900-01$ $8.956600-01$ 0.508700-0 . $534600-0$ $.534600-1$ $9.696400-01$ $9.912600-0$ 1.05120000 $1.090600 \quad 00$

$0.64000 \mathrm{BB}$ 0.00515000 0.00515000 0.00542300

0.06152502 0.00515000 0.00515000 0.00542300

1). 64000.88 1.63618000 1.63618000 2.25110000

$0.64000 \quad B B$ $9.471104-01$ 7.51728700 $7.598351-01$

.06176504 1.63618000 1.63618000 2.25110000

0.06221563 3.73964600 5.02193000 5.980839-01

$0.64000 \quad B B$ $7.974500-04$ 2.070695-0 0.06224570 $1.023250-03$ 7.055695-02 4.57572100

4.872900-02

3.52784 6.59783900 2. 286800-0 5.194100-01 5.044000-01 4. 729900-01 $4.729900-01$ $4.729900-01$ $4.790500-01$ $5.675100-01$ $4.872900-02$ $4.872900-02$ $6.511800-02$ $9.789400-02$ $9.789400-02$ $0.789400-02$ $9.295400-02$

$\operatorname{SGSO}(\mathrm{N}, \mathrm{N})$

2.073900-01 $2.302600-01$ $2.476100-01$ $2.294700-01$ $1.908300-01$ 2.854600-0 2.901900-01 3.535600-01 $4.582100-01$ $6.708400-01$ $8.768100-01$ $9.7601000-01$ 9.142000-01 8.969500-01 $8.834400-01$ $8.707500-01$ $8.729000-0$ 9.001500-01

0.00000000 0.00000000 0.00000000 0.00000000 0.00000000 0.00000000 0.000000 0.00000000

$\operatorname{SGSO}(N-1, N)$

$\operatorname{SGSO}(\mathrm{N}-2, \mathrm{~N})$

$\operatorname{SGSO}(\mathrm{N}-3, \mathrm{~N})$

0.00000000 $1.588500-02$ $1.763600-02$ $1.757600-02$ 1.4617 $1.461700-02$ $1.751600-02$ $2.222700-02$ $6.402300-03$ $8.297300-03$ $1.214800-02$ $1.587700-02$ $1.676800-02$ 3. 308300-0 $3.258500-02$ 3.211700-0 3.045700-02

REACTOR MATERIALS MAT(I), I $=1$, II

0.00515000 0.00515000 0.00542300

0.00515000 0.00515000

$0.005150 \quad 00$ 0.00542300

0.00515000

$0.005150 \quad 00$ 0.00515000

MATERIAL CONCENTRATIONS N(I), I $=1$, II

1.63618000 $1.636180 \quad 00$ .63618000

1.63618000

2.25110000

1.63618000
2.25110000

1.63618000

SURFACE SOURCE $Q(N), N=1$, NN

6.34677300

4.51774600

$\begin{array}{lll}6.850132 & 00 & 6.00211400 \\ 4.029646 & 00 & 3.28412100\end{array}$

75933400

2.04808700

SENSOR ACTIVATION CROSS SECTIONS ( $S G(N, S), N=1, N N$ ), $S=1$, SS $2.300800-03 \quad 4.218100-03 \quad 6.327100-03 \quad 9.394800-03$ $1.533846-01$ 2. $218100-03$ 4.25872301
0.00000000 3.000000 00 3.00000000 0.00000000 0.00000000 0.00000000 0.000000 0 0.00000000

$\mathrm{SGSO}(\mathrm{N}-4, \mathrm{~N})$

0.00000000 0.00000000 0.00000000 0.00000000 0.00000000 .00000000 0.00000000 0.00000000 0.00000000 0.00000000 0.00000000 0.00000000 0.00000000 . 00000 0.00000000 0.00000000 0.00000000 0.00000000 0.00000000 0.00000000

0.00000000 0.00000000 0.00000000 0.00000000 0.00000000 0.00000000 $0.000000 \quad 00$ 0.00000000

$\operatorname{SGSO}(N-5, N)$

0.00000000 0.00000000 0.00000000 0.00000000 0.00000000 0.00000000 0.00000000 0.00000000 0.00000000 0.00000000 0.00000000 0.00000000 0.00000600 $0.000000 \quad 00$ 0.00000000 0.00000000 0.00000000 0.00000000 0.00000000

0.00515000 0.00542300

0.00515000 0.00567600

1.63618000

1.63618000 $2.251100 \quad 00$

3.62436300 1.22933500

$2.782745 \quad 00$ 1.017542 .00

$1.188730-02$

$1.399635-02$
4.32 .582901

3.719577 


$\mathrm{CU}$

\begin{tabular}{|c|c|}
\hline $\begin{array}{l}1.795000-05 \\
2.159500-04 \\
1.287128-01 \\
1.770700-03 \\
4.574340-02 \\
2.28126600\end{array}$ & $\begin{array}{l}2.305000-05 \\
5.864500-04 \\
2.153601-01 \\
2.508500-03 \\
1.254254-01 \\
3.73277200\end{array}$ \\
\hline $\begin{array}{ll}0.64000 & 8 B \\
2.251100 & 00\end{array}$ & $\begin{array}{l}0.06320562 \\
2.251100 \quad 00\end{array}$ \\
\hline $\begin{array}{ll}0.64000 & 88 \\
0.000020 & 00\end{array}$ & $\begin{array}{l}0.06322725 \\
0.00002400\end{array}$ \\
\hline $\begin{array}{ll}0.64000 & 8 B \\
0.000012 & 00\end{array}$ & $\begin{array}{l}0.06325726 \\
0.00002200\end{array}$ \\
\hline $\begin{array}{l}0.64000 \quad B B \\
1.187051-01\end{array}$ & $\begin{array}{r}0.06332515 \\
-1.678744-01\end{array}$ \\
\hline $\begin{array}{ll}0.64000 & 8 B \\
1.000000 & 00\end{array}$ & $\begin{array}{l}0.06337516 \\
8.509914-01\end{array}$ \\
\hline $\begin{array}{ll}0.64000 & B B \\
0.000000 & 00\end{array}$ & $\begin{array}{l}0.06344517 \\
9.496+12-01\end{array}$ \\
\hline $\begin{array}{ll}0.64000 & 8 B \\
0.000000 & 00\end{array}$ & $\begin{array}{l}0.06351520 \\
9.496412-01\end{array}$ \\
\hline $\begin{array}{l}0.64000 \quad B \bar{~} \\
9.404323-01\end{array}$ & $\begin{array}{l}0.06353521 \\
5.083741-01\end{array}$ \\
\hline $\begin{array}{l}0.64000 \quad B E \\
6.521452-01\end{array}$ & $\begin{array}{l}0.06355522 \\
3.478548-01\end{array}$ \\
\hline $\begin{array}{ll}0.64000 & 8 B \\
0.60000 & \text { STZ }\end{array}$ & $\begin{array}{l}0.064 C 1 \quad 503 \\
0.00003\end{array}$ \\
\hline $\begin{array}{l}0.64000 \text { BB } \\
0.60000 \text { ST }\end{array}$ & $\begin{array}{l}0.06425514 \\
0.00003\end{array}$ \\
\hline $\begin{array}{l}\text { U.64000 BB } \\
0.60000 \text { STZ }\end{array}$ & $\begin{array}{l}0.06450 \quad 515 \\
0.00071\end{array}$ \\
\hline
\end{tabular}

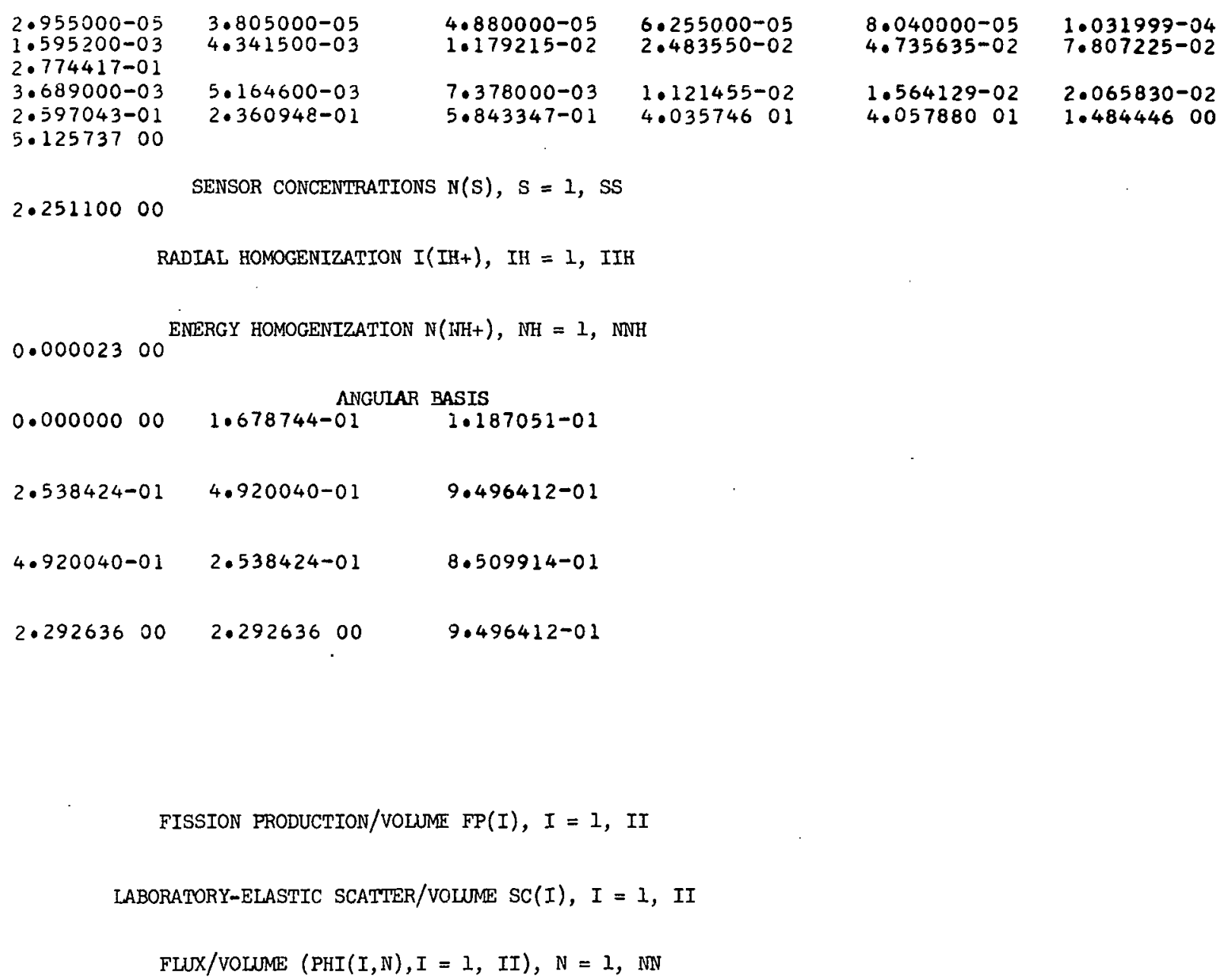


8.000000 00

1.20000001

1.60000001 $R(I)$

CONVERGE

0.00000000

3.488773-01

3.571272-01

$3.653771-01$

3.736270-01

$3.901268-01$

3. $983767-01$

$4.06626 t-01$

4.148765-01

$4 \cdot 165265-01$
$4 \cdot 181765-01$

4. $198265-01$

4. $214765-01$

4. $231264-02$

$4 \cdot 332770-01$

4. 58664t-01

5.939985-01

7.293323-01

$8.646661-01$
1.00000000 $R(I)$

9.960193-01

0.00000000

1.00000000

9.999673-01

9.999997-01 DV (I- $) / 2$

0.00000000

3.042884-02

$1.456118-03$

$1.490148-03$

$1.524179-03$

$1.558209-03$

$1.592240-03$

$1.626267-03$

$1.660301-03$

$1.594331-03$

$3.429437-04$

$3.456711-04$

$3.470323-04$

$3.483936-04$

$2.173250-03$

5.661061-03

.561523-02

$4.477286-0$

$5.393047-02$
$6.308810-02$

$\mathrm{DV}(\mathrm{I}-) / 2$

0.00000000

$3.042884-02$

$3.488773-01$

$3.571272-01$

$3.571272-01$

$3.653771-01$

$3.736270-01$

$3.818760-01$

$3.901268-01$

$3.983767-01$

$4.066266-01$

$4 \cdot 148765-01$

$4.16526 \equiv-01$

$40181765-01$

$4.198265-01$

$4.214765-01$

4.2147

4.231254-01

$4 \cdot 332770-01$

$4.586646-0$

$5.939985-01$

7.293323-01

5.646661-vi

1. 00000000

PHI $(I, 7)$

$3.716 € 3400$

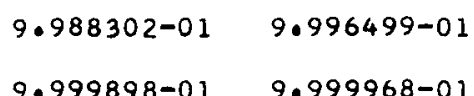

$9.999999-01 \quad 1.00000000$ FP(I-)

0.00000000

0.00000000

0.00000000

0.00000000

0.00000000

0.00000000

0.00000000

0.00000000

0.00000000

0.00000000

0.00000000

0.00000000

0.00000000

0.00000000

0.00000000

0.00000000

0.00000000

0.00000000

0.00000000

0.00000000

$\operatorname{PHI}(I, 1)$

7.798649-01

$8.289417-01$

8.312213-01

$8.336944-01$

$8.363932-01$

$8.393565-01$

$8.426310-01$

$3.462725-01$

$8.503474-0$ ]

$8.549346-01$

$8.559192-01$

8.569287-01

8.579638-01

8. 590254-01

$8.601145-01$

$8.628481-01$

8.725665-01

$8.952271-01$

9.129242-01

9.177059-01

$\operatorname{PHI}(I, 9)$

$7 \cdot 32141600$ $\mathrm{FP}(\mathrm{I}+)$

0.00000000

0.00000000

0.00000000

0.00000000

0.00000000

0.00000000

0.00000000

0.00000000

0.00000000

0.00000000

0.00000000

0.100000000

0.00000000

0.00000000

0.00000000

0.00000000

0.00000000

0.00000000

0.00000000

0.00000000

$\mathrm{PHI}(I, 2)$

3.21092000

3.36687400

3.37411500

3.38195300

3.39048500

3.39983200

3.41013500

3.42156500

3.43432400

3.44865300

3.45172500

3.45487200

3.45809800

3.46140500

3.46140500

3.47324400

3.50477900

.3 .5 .7621600

3.61050600

3.63197200

3.64703400

$\operatorname{PHI}(I, 10)$

4.53162100
0.00000000
LEAKAGE

9.861240-01 -7.744675-01

$9.998936-01-7.523721-01$

9.999990-01 -7.522018-01

$1.00000000 \quad-7.522002-01$ $A$ (I, IN)

3.68735300

3.44108901

3.88367001

4.41913201

5.08469601

5.91223301

$6.963748 \quad 01$

8.35034501

1.04618502

1.41718402

1.525431 .02

1.65251302

1.80339302

1.99685202

$2.289616 \quad 02$

2.35711102

2.66650202

3.19100902

3.33609002

3.42326402

3.48328902

$\operatorname{PHI}(I, 3)$

5.75004000

5.92875600

5.93706500

5.94602500

5.95574300

5.96634700

5.97799100

5.99086100

6.00517200

6.02118400

6.02460900

6.02811600

6.03170900

6.03538800

6.03915900

6.04850400

6.08439100

6.16413800

6.20244300

6.22643000

6.24326200

PHI $(I, 11)$

3.33859400
$A(I, C U)$

3.542635-02

8.968609-02

9.566074-02

$1.031736-02$

$1.129365-01$

$1.260968-01$

$1.450948-01$

$1.761645-01$

$2.3875 .91-01$

4.196949-01

4.848881-01

5.726139-01

$6.913081-01$

$9.054817-01$

1.43599800

1.48131400

1.68683600

2.03048300

2.12413100

2.18021400

.21876900

$\operatorname{PHI}(I, 4)$

6.59472300

6.67503700

6.67880800

6.68284300

6.68718700

6.69189100

6.69701600

$6.702636 \quad 00$

6.70883600

6.71718200

6.72021200

6.72177800

6.72338100

6.727567 .00

6.727567 .00

6.74159100

6.77420900

6.79004100

6.79999800

6.80699900

PHI $(I, 12)$

2.03652500

SCATTER

SC

1.80725200

1.81743000

1.81751500

1.81751600 $A(I, A U)$

5.35271000

3.32489301

3.733889 ol

4.22831001 
3.71473100 $3.71267 \in$ 00 3.70801200 3.70533500 3.70237200 3.69907100 3.69537100 $\begin{array}{lll}3.695371 & 00 \\ 3.694578 & 00\end{array}$ 3.694578 00 3.69376600 3.69253400 3.69208200 3.69123700 3.68906000 3.68113700 3.66393200 3.65564600 3.65045100 3.64680400 PHI $(I, 15)$ 0.0 Covuo 00 4.999923-02 $6.241685-02$ $9.875624-02$ $1.252372-01$ $1 \cdot 25<372-01$ $2.052153-0$ $2 \cdot 787652-0$ 4. $135265-0$ $4.561753-01$ 5.066343-01 $5.668853-01$ ó.395018-01 7.278330-01 $7.511700-01$ 3.503871-01 1.01918500 1.06573900 1.09379400 1.11307700 $J(I, 4)$

4.569429-09 -6.057509-02 -0.189722-02 $-6.321169-02$ $-6.451766-02$ $-6.581411-02$ $-6.709969-02$ $-6.837364-02$ -ó.963379-02 $-7.112531-02$ $7.112531-02$

$-7.161690-02$
2.84608800 2.84316800 2.84152700 2.83973700 2.83777300 2.83560200 2.83318000 2.83318900 2.83267400 2.83214800 2.83161000 2.83105500 2.83049500 2.82923800 2.82322800 2.81080100 2.80488700 2.80119700 2.79861300 PHI $(I, 16)$ 0.00500000 $1.150045-02$ $1.625267-02$ $3.318470-02$ $40802152-02$ $7.023572-02$ $1.040708-01$ $1.040708-01$ 1.566814-01 2.584757-01 2.934837-01 $3.387305-01$ $4.021204-01$ 4.861282-01 $5 \cdot 99940 t-01$ $6.200<23-01$ $7.024836-01$ $8.432477-01$ 3.819347-01 9.051657-01 9.211589-0 I $J(I, 5)$

0.00000000 9.342014-04 1.051109-03 1.174663-03 1. 305456-03 $1.444239-03$ $1.591893-03$ F. 749427-03 $1.918056-03$ $2.099180-03$ 2.137020-03 $2.175424-03$ 2. $214430-03$
7.32551300 7.33479900 7.34011700 7.34599600 7.35254000 7.35987400 7.36815000 7.36992900 7.37175400 7.37362600 7.37362600 7.37554800 7.37752100 7.38229300 $7.399276 \quad 00$ $7.434946 \quad 00$ 7.45216800 7.45298400 7.46297400 $\operatorname{PHI}(I, 17)$

0.00000000 $1.096413-03$ $1.903460-03$ $3.339403-03$ 5.902343-03 $1.055454-02$ 1. $914320-0$ 1. 544707-0 $6.575714=02$ $1.359101-01$ $1.562243-01$ $1.910117-01$ $2.377752-01$

3.165532-01 $4.453560-01$ $40604996-01$ $5.229682-01$ $6.292225-01$ 6.582589-0 0.756757-01 0.876584-0 $J(I, 6)$

5.886992-09 $2.739084-02$ 2.808872-02 $2.879024-0$ ? $2 \cdot 949569-0$. 3.020553-02 $3.092022-02$ 3.164033-02 $3.236654-02$ 3.309963-02 3.324712-02 3.354309-02
4.54202100 4.56551700 $+.57893200$ 4.59373600 4.61019200 4.62862300 4.64941600 4.65388800 4.65847500 4.66318300 4.66801600 4.67297900 4.068388300 4.73101900 4.31970900 4.86218800 4.88875800 4.90739300 PHI (I,18)
$0.557060-08$ Q.557060-08
$2.201791-05$ 5.509941-05 1.400551-04 3.669622-04 $9.703538-04$ 2.628707-0 $7.215296-03$ 2.054821-02 $6.747030-02$ $8.428275-02$ 1.057918-0 $1.363307-0$ $1.983086-01$ 3.465837-01 $3.582537-01$ 4.092458-01 4.945544-01 5.176406-0 5.314573-0

5.409530-01$$
\mathrm{J}(\mathrm{I}, 7)
$$

$3.602278-09$ 3.079061-02 $3.153287-02$ 3.227611-02 3.302041-02 3. 376585-02 3.451253-02 3.526055-02 3.601003-02 $3.576114-02$ 3.691156-02
3.36379100 3.42120500 3.45427500 3.49101800 3.53219400 3.57875000 3.63186700 3.63186700 3.64336800 3.65519800 3.66737200 3.67990400 3.69280800 3.71614000 3.84736000 4.05473500 4.15273300 4.21368600 4.25632800 PHI $(I, 19)$

0.00000000

4.693457-06 1.511203-05 4.978763-05 $1.645917-04$ $6.136038-04$ $2.340593-03$ $9.211497-03$ 3.692318-02 $1.779842-01$ 2.355150-01 3.138779-01 4.217219-01 6.344077-01 1.25135400 1.29292900 1.48302500 1.79792500 1.88254700 1.93311100

1.96783500 $J(I, 8)$

$6.587817-10$ 2.123692-02 2. $172910-02$ 2.222052-02 2.271109-02 2.320067-02 2.368913-02 $2.417626-02$ $2.466190-02$ $2.514577-02$ 2.524231-02

2.543514-02

2.07938300 2.18024300 2.24015700 2.30828300 2.38667100 2.47806200 2.58611400 2.61002000 2.63481600 
$-7.186154-02$ $-7.210541-02$

$-7.041619-02$

-6.670993-02

$-5.151107-02$

$-4 \cdot 195277-02$

$-3.538651-0$

$-3.059754-02$ $J(I, 12)$

$1.646954-10$

-5.118382-01

$-50317480-0$

0

$-5.522699-01$
$-5.734953-01$

$-5.955345-01$

$-6.185243-01$

$-6.426371-01$

-6.680932-0

$-6.787020-01$

$-6.841094-01$

$-6.895692-01$

$-6.951445-01$

$-6.788591-01$

$-6.417321-01$

$-4.561089-0$

-3.714740-0

$-2.709280-01$

$0.64000 \mathrm{BB}$

0.60000 STZ

$\operatorname{NUSGF}(\mathrm{NH})$

0.64000 BB

0.00000000

0.00000000

PHI (NH)

4.60001501

7.05022900

$2.216915-01$

$0.64000 \mathrm{BB}$

0.60000 STZ

0.64000 BB
0.60000 STZ

PHI (NH)

4.70602101

1.52824201

1.85789200

$0.64000 \mathrm{BB}$

0.60000 STZ
$2.254063-03$ $2.294297-03$ 2.240537-03 $3.784115-03$ $2.921944-03$ $2.379724-03$ $2.007242-03$

i. $735571-03$ $J(I, 13)$

0.00000000

$-3.644528-01$

$-3.929212-01$

$-4 \cdot 231383-01$

$40554322-01$

$4.554322-01$

$50279603-01$

(1)

$5.665123-01$

$0.058013-0$

$.475830-01$

$0.562425-01$

$6.050035-01$

$6.738656-01$

$-6.828282-01$

$0.828282-01$

$6.756813-01$

$0.756813-01$

$0.229473-01$

$3.869857-01$

-151772-01

$2.658470-01$
$-2.298689-01$

0.11521613

0.00001

SG(NH)

0.11524630

$8.444276-01$
3.08819500

3.08819500

$\mathrm{L} / \mathrm{V} / \mathrm{PHI}$

$-5.424340-02$

$-1.70627000$

$-8.38744200$

0.11521613 0.00001

0.11524630 0.00003

$\mathrm{L} / \mathrm{V} / \mathrm{PHI}$

$2.950821-02$

$5.583716-02$

0.11521613

0.00002
$3 \cdot 369155-02$ $3.384037-02$ $3.304756-0$ $3.140076-02$ $2.424653-02$ $2.424653-02$ $1.665657-02$ $.440234-02$ $J(I, 14)$

0.00000000

$-1.413925-01$

$-1.601624-01$

$-1.815390-01$

$-2.055038-01$

$-2.055038-01$

$-2.642969-01$

$2.042969-01$

$-3.424455-01$

$-3.900538-01$

$-4.003657-01$

$-4 \cdot 109596-01$

$-4 \cdot 218408-01$

$-4 \cdot 330147-01$

$-4.444860-01$

$-4 \cdot 239297-0$

$-3.892707-0$

$-2.415327-0$

$-1.967143-01$

$-1.659254-01$

$-1.434701-01$

$623344-02$

$148013-02$

VOLUME SOURCE $\mathrm{Q}(\mathrm{NH}), \mathrm{NH}=1, \mathrm{NNH}$

$\mathrm{SGSO}(\mathrm{NH}, \mathrm{NH})$

7.901842-01

1.04705300

1.43431000

DIFFUSOR $\mathrm{D}(\mathrm{NH})$

$6.442347-02$

$5.694999-02$

$-1, N$ )

$0.000000 \quad 00$

$6.805480-03$

$5.469278-04$

ALBEDO A(NH)

$4.047042-01$

$2.697798-01$

$\mathrm{SGSO}(\mathrm{NH}-2, \mathrm{NH})$

0.00000000

0.00000000

0.00000000 $\mathrm{SGSO}(\mathrm{NH})$

$7.969896-01$

1.04760000

1.43431000

VOLUME SOURCE $\mathrm{Q}(\mathrm{NH}), \mathrm{NH}=1, \mathrm{NNH}$

PROBABILITY/PATH

( 3 rows of zero probabilities for void)

DIFFUSOR D(NH)

4.074492-02

$5.052405-02$

$9.780125-01$

7.608522-01

SGSO(NH)

0.00000000

$7.283748-01$

SGA (NH)

0.00000000 Note effect of using

$0.00000000\}$ too coarse a lattice

in a vold region

SGSO (NH-5, NH)

(empty)

0.0000

0.00000000

0.00000000

$4.743794-0$

2.04059500

1.09881002

$0.00000000<$

0.000000

0.00000000

VOLUME SOURCE $Q(\mathrm{NH}), \mathrm{NH}=1, \mathrm{NNH}$

$<$

$<$ 


\begin{tabular}{|c|c|c|c|c|}
\hline & \multicolumn{2}{|l|}{$F(N$} & $\mathrm{SG}(\mathrm{NH})$ \\
\hline BAND & $\mathrm{NH}$ & 0.64000 & $B B$ & 0.11524630 \\
\hline & 1 & 0.000000 & 00 & $1.744701-01$ \\
\hline & 2 & 0.000000 & 00 & $3.380183=0$ \\
\hline & 3 & 0.000000 & 00 & 82747 \\
\hline IND & NH & PHI (NH) & & $\mathrm{L} / \mathrm{V} / \mathrm{PHI}$ \\
\hline & 1 & 4.683721 & 01 & -1.120744 \\
\hline & 2 & 1.355059 & 01 & $-1.604813-01$ \\
\hline & 3 & 1.513679 & 00 & $-2.043587-0$ \\
\hline
\end{tabular}

\begin{tabular}{|c|c|c|c|}
\hline \multirow{2}{*}{$\begin{array}{l}\mathrm{SGSO}(\mathrm{NH}, \mathrm{NH}) \\
1.632627-01 \\
1.146051-01 \\
4.419255-02\end{array}$} & \multicolumn{2}{|c|}{$\begin{array}{l}\text { PROBABILITY/PATH } \\
\mathrm{SGSO}(\mathrm{NH}-1, \mathrm{NH})\end{array}$} & \multirow{2}{*}{$\begin{array}{l}\text { SGSC (NH-3, NH) } \\
\text { (empty) } \\
0.00000600 \\
0.00000000 \\
0.00000000\end{array}$} \\
\hline & $\begin{array}{l}00.00 \\
04-03 \\
96-05\end{array}$ & $\begin{array}{ll}0.000000 & 00 \\
0.000000 & 00 \\
0.000000 & 00\end{array}$ & \\
\hline $\begin{array}{l}\text { IFFUSOR D(NE) } \\
5.924767-01 \\
6.054866-01 \\
6.026511-01\end{array}$ & $\begin{array}{l}A(N H) \\
25-01 \\
22-01 \\
48-01\end{array}$ & $\begin{array}{c}\text { SGSO }(\mathrm{NH}) \\
1.646688-01 \\
1.146650-01 \\
4.419255-02\end{array}$ & $\begin{array}{l}1321-03 \\
3532-01 \\
5544-01\end{array}$ \\
\hline
\end{tabular}

$\mathrm{SGSO}(\mathrm{NH}-4, \mathrm{NH})$ 0.000000 (empty)

0.00000000 0.00000000

$\mathrm{SGSO}(\mathrm{NH}-5, \mathrm{NH})$ (empty) $0.00000000<$ $0.00000000<$ $0.00000000<$ 
ADJOINI

ITERATION 4.00000000

8.00000000

$1.200000 \quad 01$

1.30000001 $R(I)$

CONVERGE

LAST FOUR EIGENVALUES ORDERED CHRONOLOGICALLY

HPPP

$0.000000 \quad 00$

$1.000000 \quad 00$

$1.00002300 \quad 1.00000800$

1.00006000

1.00000100

1.00000000 $\mathrm{DV}(\mathrm{I}-) / 2$

0.00000000

3.042884-02

1.456118-03

$1.490148-03$

$1.524179-03$

$1.558209-03$

$1.592240-03$

$1.626267-03$

$1.660301-03$

$1.694331-03$

3.429487-04

3.443130-04

$3.456711-04$

$3.470323-04$

3.483936-04

$2.173250-03$

2.173250-03

$5.661061-03$
$3.561523-02$

$3.561523-02$
$4.477286-02$

$4.477286-02$
$5.393047-02$

$6.308810-02$

DV(I-)/2

$3.042884-02$

$3.042834-02$

$18-03$

$1.490148-03$

$1.524179-03$

$1.558209-03$

$1.592240-03$

$1.626267-03$

$1.660301-03$

$1.694331-03$

3.429487-04

$3.443130-04$

3.443130-04

$3.456711-04$

$3.470323-04$
$3.433936-04$

$2.173250-03$

$5.661051-03$

3.561523-02

$4.477286-02$

$5.393047-02$

$6.308810-02$

PHI* $(1,12)$

1.98442100

$1.00000000 \quad 1.00000000$

1.00000000 FP* (I-)

0.00000000

0.00000000

0.00000000

0.00000000

0.00000000

0.000000

0.00000000

0.00000000

0.00000000

0.00000000

0.00000000

0.00000000

0.00000000

$0.000000 \quad 00$

0.00000000

0.00000000

0.0000000

0.00000000

0.00000000

$0.000000 \quad 00$

0.00000000

0.00000000

0.00000000

PHI*(I, 19)

$4.640685-06$

$4.640685-06$

$4.945626-05$

$1.638514-04$

$6.113493-04$

2.334879-03

$9.192662-03$

$3.6880 / 43-02$

$1.778295-01$

$2.353212-01$

3.136332-01

4. $214100-0$

$6.338380-0$

1.25003800

1.29149200

1.48185700

1.79759900

1.88232500

1.93293700

1.96769000

PHI* (I, 11)

3.25684300
1.00000000 FP* (I+)

0.00000000

0.00000000

0.00000000

0.00000000

0.00000000

0.00000000

0.00000000

0.00000000

0.00000000

0.00000000

0.00000000

0.00000000

0.00000000

0.00000000

0.00000000

0.00000000

0.00000000

0.00000000

0.00000000

0.00000000

0.00000000

PHI* (I, 18)
4.364233-0。

1.988176-05

$5.145385-05$

1.346166-04

3.575022-04

9.579780-04

2.616968-03

7.209237-03

$6.779660-02$

8.470503-02

$1.063345-01$

$1.372654-01$

$1.997002-01$

3.495397-01

3.615113-01

$4.118960-01$

$4.952956-01$

$5 \cdot 181445-01$

$5.41207-01$

PHI* $(I, 10)$

4.38462800
$1.00013300 \quad-6.807335-01$

$1.00000200 \quad-6.809587-01$

$1.000000 \quad 00 \quad-6.809638-01$

$1.00000000 \quad-6.809638-01$

$A *(I, I N)$

$A^{*}(I, C U)$

$\begin{array}{lll} & \\ 3.3689465 & 01 & 3.373326-02 \\ 3.3685165 & 8.765205-02\end{array}$

$3.80959101 \quad 9.364270-02$

$4.34881201 \quad 1.011976-01$

5.01386901 1.109769-01

5.841151 01 $1.241539-01$

$6.89329801 \quad 1.432024-01$

$.27716601 \quad 1.743507-01$

$1.03930102 \quad 2.370103-01$

$1.41078502 \quad 4.180403-01$

$1.51917602 \quad 4.832399-01$

$1.64641702 \quad 5.709955-01$

$1.79748502 \quad 6.898407-01$

$1.99108902 \quad 9.040364-01$

$2.28399902 \quad 1.43477600$

$2.35304502 \quad 1.48030800$

$2.66296402 \quad 1.68606300$

$\begin{array}{lll}.18998102 & 2.03018700\end{array}$

$3.33536402 \quad 2.12390600$

$\begin{array}{llll}3.422688 & 02 & 2.180029 & 00 \\ 3.482804 & 02 & 2.218612 & 00\end{array}$

PHI* (I 17)

PHI*(I, 17$)$

$1.054506-03$

$1.848267-03$

$3.261916-03$

$5.798937-03$

$1.041210-02$

$1.894571-02$

$3.517756-02$

$6.739545-02$

$1.354325-01$

$1.556123-01$

1. $903740-01$

2.370765-01

.156682-01

$4.442275-01$

4.593674-01

$5.220447-01$

6.289638-01

6.580831-01

$6.755385-01$

6.875440-01

PHI* $(I, 9)$

7.06037500

PHT* $(I, 16)$

0.000000

$1.124847-02$

$2.278629-02$

.277062-02

4.753259-02

$6.965977-02$

$1.034006-01$

$1.559114-01$

2.574684-01

2. $924475-01$

$3.375874-01$

4.009234-0

4.848543-01

$5.985338-01$

$0.013561-01$

$8.429478-01$

8.817308-01

$9.050076-01$

$9.210262-01$

3.51 PH (I,8)

3.30097600

$8.575896-01$

\begin{tabular}{cccc}
$\begin{array}{c}\text { SCATTER } \\
\text { SC* }\end{array}$ & \multicolumn{2}{c}{ FISS PROD } \\
1.827431 & 00 & 0.000000 & 00 \\
1.827258 & 00 & 0.000000 & 00 \\
1.827255 & 00 & 0.000000 & 00 \\
1.827255 & 00 & 0.000000 & 00
\end{tabular}

$A^{*}(I, A U)$

5.13420900

3.25436601

3.66233201

4.16039101

4.774175 ol

5.53661401

6.50419101

7.77146401

9.70529601

1.30761902

1.40540302

1.51951702

1.65406902

1.82396402

$2.073762 \quad 02$

2.13601602

2.42430102

2.88763602

3.01845202

3.09704102

3.15115102

PHI* (I, 15)

4.900651-02

6.135441-02

$7.717481-02$

9.755219-02

1.239667-01

$2.038592-01$

2.772821-01

4.120577-01

4.547435-01

5.052428-01

$5.655331-01$

$6.381766-01$

7.265056-01

$8.501897-01$

1.01896000

1.06563600

1.09367400

1.11297700

PHI* (I,7)

3.53253800

5. PHI* 07 (I, 14$)$

2.347378-01

2.658082-01

3.030261-01

$3.480117-01$

.707634-01

$5.585297-01$

6.857938-01

9.397648-01

9.980039-01 
9.068009-01 $9.635600-01$ 1.02931700 1.10633700 1.19927500 1.32286100 1.382265 o0 1.69335600 1.74333100 $\begin{array}{ll}1.743331 & 00 \\ 1.796567 & 00\end{array}$ 1.85335400 1.91400800 1.97887700 2.0132050 2.27562900 2.72368000 2.84787100 2.9225 2.97397000 5. PHI* 652683 (I, $)_{0}$ 5.75910200 5.76407400 5.76944000 5.77526600 5.78163100 $5.78862 \% 00$ 5.79637300 5.80499900 5.81456500 5.81673500 5.81895500 5.82102700 5.82325300 5.82553400 5.83129100 5.85039300 5.89630500 5.91844800
5.93233600 5.94209000

$0.000000 \%$ $-8.248909-03$ $-1.161 \in 68-02$ $-1.640885-02$ $-2.327304-02$ $-3.317950-02$ $-4.761610-02$ $-6.889662-02$ $-1.007105-01$ $-1.015642-01$ $-1.895533-01$
2.02859300 2.13247100 2.19412100 2.26416600 2.34467500 2.43841200 2.54905200 2.57350500 2.59885700 2.62515400 2.65244700 2.68078900 2.71687400 2.96202300 3.37028200 3.51401000 3.60132300 3.66187000 $6.577^{*}(I, 4)$ 6.066166800 6.66553300 6.66969100 6.67419100 6.67909100 6.68446000 6.69038200 6.69695300 6.70429000 6.70535800 6.70746200 6.70910500 6.71078700 6.71251000 6.71686800 6.73241500 6.76803700 6.78521700 6.79599300 6.80356000 $-\mathrm{J} *(I, 15)$ $-3.384990-02$ - $30384990-02$ $-5.235240-02$ $-6.534907-02$ $-8.182925-02$ $-1.028373-01$ $-1.297757-01$ $-2.103336-01$ $-2.208297-01$ $-2.208297-01$ $-2.435290-01$
3.28416600 3.31382200 3.34620300 3.36181000 3.42127300 3.46538200 3.46538200 3.57170400 3.58393600 3.59651100 3.60344300 3.62274800 3.63644000 3.66126000 3.80125800 4.02277900 4.02277900 4.12749500 4.19264800 4. 23823500 6. $344{ }^{*}{ }^{2}\left(I_{6} 3\right.$ 6o 6.34775400 6.34790600 6.34804500 6.34816900 6.34827400 6.34841100 6.34842900 6.34840200 6.34839100 6.34837700 6.34836100 6.34834200 6.34832100 6.34837000 6.34798600 6.34743400 6.34725000 6.34715600 6.34709700 $-J *(I, 14)$ 0.000000 $-1.413661-01$ $-1.601963-01$ $-1.814331-01$ $-2 \cdot 054645-01$ $-2.329538-01$ $-2.644555-01$ $-3.006463-01$ $-3.427880-01$ $-3.905910-01$ $-4 \cdot 009448-01$ $-4 \cdot 115805-01$
4.39832700 4.41309400 4.42911100 4.44659700 4.46582100 4.48711300 4.48711300 4.51086900 4.53756700 4.54329500 4.549168 00 4.55519100 4. 56136800 4.56770700 4.58156300 4.64318100 4.643181
4.758686 00 4.81399300 4.84858200
4.87284000

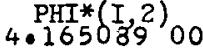
4.04077500 4.03501200 4.02876100 4.02194300 4.01445900 4.01445900 4.00619300 3.9700400 3.98672600 3.97516000 3.97267800 3.97013300 3.96752400 3.96484900 3.96210400 3.95532000 .92968800 3.87179000 3.84404100 3.82668000 3.81450200 $-J *(I, 13)$

0.00000000 $-3.665390-01$ $-3.952665-01$ $-4.257684-01$ $-4.583725-01$ $-4 \cdot 934783-01$ $-5.313290-01$ $-6.593135-01$ $-6.681282-01$ $-6.770422-01$
7.07000800 7.08038300 7.09162400 7.10388300 7.11734100 7.13221700 7.14877500 7.16732800 7.17130100 7.17537100 7.17954200 7.18381700 $7.188200 \quad 00$ 7.19848800 7.23947300 7.32384900 7.364405 00 7.38980800 7.40763600

PHI* (I, I)

1.32259800 1.31514200 1.30709300 1.29835300 1.28880600 1.27830900 1.26669800 1.25377200 1.23929700 1.23619900 1.23302700 1.22977700 1.22644700 1.22303500 1.22303500 1.18365300 1.11190300 1.07737500 1.05573800 1.04054900 $-J *(I, 12)$ $1.307052-09$ $-5.061865-01$ $-5.260266-01$ $-5.464029-01$ $-5.673914-01$ $-5.890810-01$ $-6.115784-01$ $-6.350111-01$ $-6.595370-01$

$-6.853561-01$ $-6.906918-01$ $6.960909-01$

3.29128900 3.28071500 3.26910400 3.24200600 3.24200600 3.22602600 3.20801200 3.18757100 3.18316300 3.17863600 3.17398700 3.16921000 3.16430000 3.15322400 3.10590600 3.10590600 3.00657100 2.959353 00 2.92991300 0.00000000 $-4.090867-06$ $-1.336934-05$ 


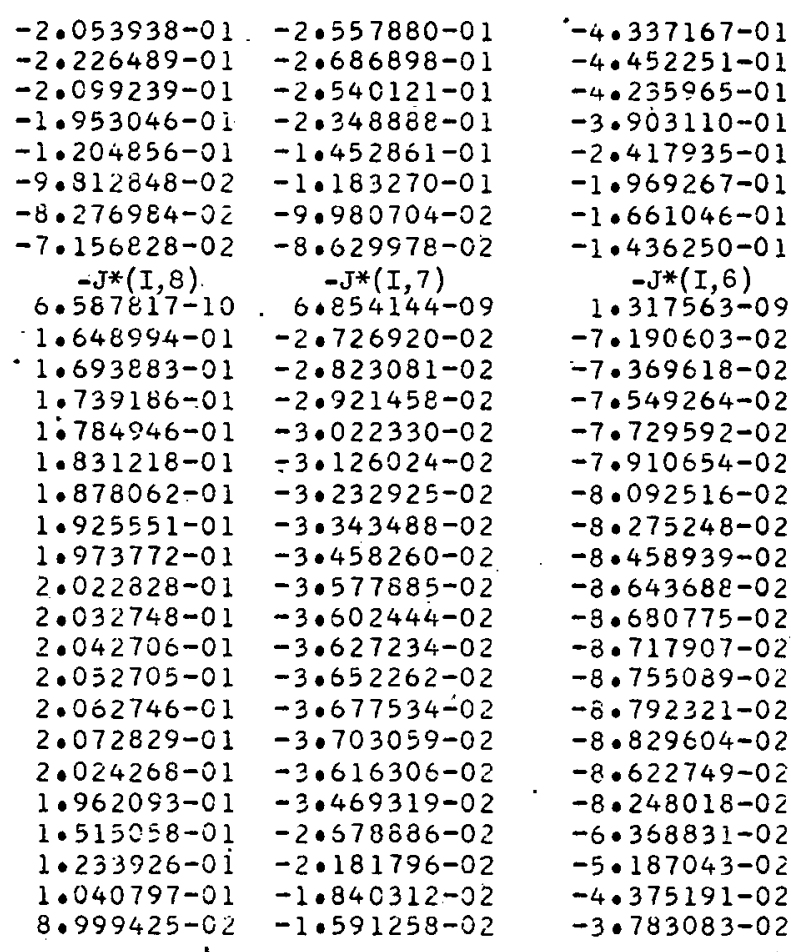

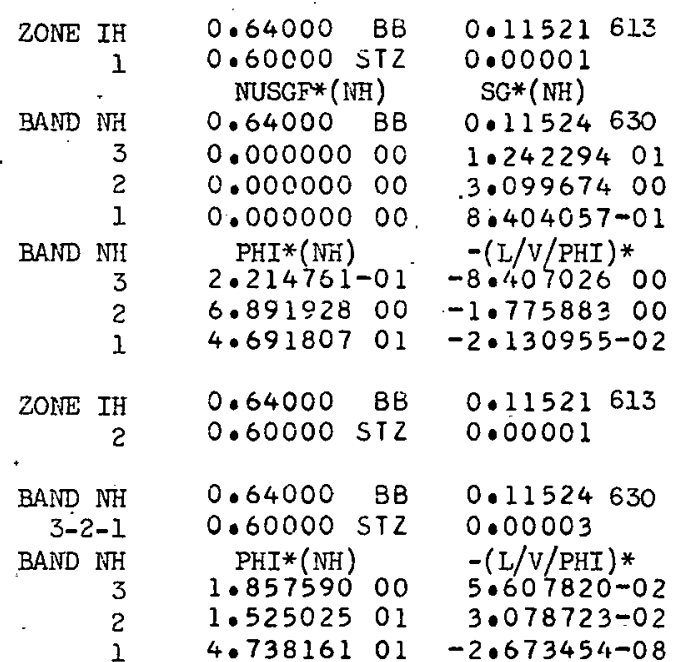

CELL IIH 0.64000 BB 0.11521613 0.60000 STZ 0.00001 .
$-6.860544-01$

$-6.951637-0$

$-6.786439-0$

$-6.243891-01$

$-3.873472-01$

$-3.154716-01$

$-2.660954-01$

$-2.300837-01$

$-\mathrm{J} *(1,5)$
$1.317563-09$

$1.317563-09$
$-8.182415-02$

$-8.182415=02$

$-8.378478-02$

$-3.574723-02$

$-8.77 .1166-02$

$-8.967824-02$

$-9.164716-02$

$-9.361866-02$

$-9.559301-02$

$-9.757052-02$

$-9.796642-02$

$-9.836247-02$

$-9.875866-02$

$-9.915500-02$

$-9.955149-02$

$-9.721925-02$

$-9.283394-02$

$-7.168309-02$

' $-5.838172-02$

$-5.838172-02$

$-4.924407-02$

VOLUME SOURCE $Q(\mathrm{NH}), \mathrm{NH}=\mathrm{NNH}, 1$

$\mathrm{SGSO}^{*}(\mathrm{NH}, \mathrm{NH})$ PROBABILITY/PATH

$\mathrm{SGSO} *(\mathrm{NH}+\mathrm{l}, \mathrm{NH})$

$\mathrm{SGSO}^{*}(\mathrm{NH}+2, \mathrm{NH})$

1.43435900

.01525900

8.144417-01

DIFFUSOR $D^{*}(\mathrm{NH})$

$6.435593-02$

$9 \cdot 788826-02$

$0.000000 \quad 00$

$5.926925-02$

3.168603-02

ALBEDO $A^{*}(\mathrm{NH})$
$2.687839-01$

3.949045-0

9.807655-01

0.00000000 0.00000000 0.00000000 $\mathrm{SGSO} *(\mathrm{NH})$

1.49362800

$8.144417-01$

VOLUME SOURCE $Q(\mathrm{NH}), \mathrm{NH}=\mathrm{NNH}, 1$

PROBABILITY/PATH

( 3 rows of zero probabilities for void)

DIFFUSOR D*(NH)
$5.056935-02$ $5.056935-02$

$4 \cdot 133445-02$

ALBEDO A* $(\mathrm{NH})$

$7.577335-0$

9.911729-01

.000000

0.00000000

0.00000000

VOLUME SOURCE $Q(\mathrm{NH}), \mathrm{NH}=\mathrm{NNH}, I$
$-2.511426-01,-1.831149-01$ $-2.521976-01-1.838493-01$ $-2.462892-01 \quad-1.795422-01$ $-2.331611-01-1.705809-01$ $-1.800388-01-1.317166-01$

$-1.466311-01-1.072755-01$

$-1.236810-01 \quad-9.048523-02$

$-1.069428-01-70823954-02$ $0 *(T, 2)$ $6.854(1,2)$

- $0.963266-02$

$9.963266-02$

$1.021420-01$

$1.046622-01$

$1.071943-01$

$1.097395-01$

1.122993-01

$1.148756-01$

$1.174705-01$

$1.200863-0$

1. 206121-01

$1.211390-01$
$1.216669-01$

$1.221957-01$

$1.227257-01$

$1.198505-01$

1. 155472-01

$8.922152-02$

$7.266570-02$

$6.129236-02$

5.299743-02

$823954-0$
$-J *(I, 1)$

$1.471748-09$

$1.262907-01$

1.293241-01

$1.323608-01$

$1.354010-01$

$1.384452-01$

$1.414937-01$

$1.445468-01$

$1.476052-01$

$1.506693-01$

$1.512828-01$

1.518967-01

$1.525107-01$

1.531251-01

.1.537398-01

1.501380-01

1.442238-01

1. $113645-01$

9.069991-02

$7.650395-02$
$6.615037 \div-02$

$\mathrm{SGSO} *(\mathrm{NH}+4, \mathrm{NH})$

$$
\text { (empty) }
$$

$(\mathrm{NH}+3, \mathrm{NH})$

$$
\text { (empty) }
$$

0.00000000

0.00000000

0.00000000

$\mathrm{SGA} *(\mathrm{NH})$

1.09293101
2.05272900

$2.596404-02$

$\mathrm{SGSO} *(\mathrm{NH}+5, \mathrm{NH})$

$0.00000000 \quad 0.00000000<-$

0.00000000

$0.000000 \quad 00$ 
PROBABILITY/PATH

\begin{tabular}{|c|c|c|}
\hline NUSGF* (N & & $S G *$ (NH) \\
\hline $\begin{array}{l}0.64000 \\
0.000000 \\
0.000000\end{array}$ & $\begin{array}{l}B B \\
00 \\
00\end{array}$ & $\begin{array}{l}0.11524630 \\
3.824631-01 \\
3.331001-01\end{array}$ \\
\hline $\begin{array}{r}0.000000 \\
\text { PHI*(NH) } \\
1.513395 \\
1.349188 \\
4.728410\end{array}$ & $\begin{array}{l}00 \\
01 \\
01\end{array}$ & $\begin{array}{l}1.754302-01 \\
-(\mathrm{L} / \mathrm{V} / \mathrm{PHI})^{*} \\
-2.044739-01 \\
-1.633628-01 \\
-4.448278-03\end{array}$ \\
\hline
\end{tabular}

$\begin{array}{cc}\text { SGSO* }(\mathrm{NH}, \mathrm{NH}) & \text { SGSO*(NH+1, NH) } \\ 4.415937-02 & 0.00000000 \\ 1.091027-01 & 1.824712-03 \\ 1.700104-01 & 3.405073-03 \\ \text { DIFFUSOR D*(NH) } & \text { ALBEDOA*(NH) } \\ 6.025249-01 & 7.282118-01 \\ 6.052011-01 & 7.577335-01 \\ 6.010203-01 & 9.911729-01\end{array}$

$\mathrm{SGSO} *(\mathrm{NH}+2, \mathrm{NH})$

$\mathrm{SGSO}^{*}(\mathrm{NH}+3, \mathrm{NH})$

(empty)

0.00000000

0.00000000

0.00000000

0.00000000

0.00000000

0.00000000

$\mathrm{SGA} *(\mathrm{NH})$

(empty)

0.00000000

0.00000000

$\mathrm{SGSO} *(\mathrm{NH}+5, \mathrm{NH})$

$\mathrm{SGSO}^{*}(\mathrm{NH})$

0.00000000

(empty)

$0.00000000<$

4.598408-02 3.364790-01

$\begin{array}{llll}6.052011-01 & 7.577335-01 & 1.125077-01 & 2.205923-01 \\ 6.010203-01 & 9.911729-01 & 1.700104-01 & 5.419858-03\end{array}$

$0.00000000<$ 


\section{$\underline{2.3 \text { PROGRAM } S_{2} \text { LISTING }}$}

Development work in extending the capabilities of the program S sequence is still too far from completion to make a complete program repurt desirable or possible at this time. A formal record of each program released for engineering use definitely is needed, however, to provide users of the program with a basis for detailed trouble-shooting of malfunctioning cases. A direct listing of the program seems to provide the best interim integration of these requirements. Table 2 is a complete listing of program $S_{2}$. Anyone in possession of Carlson's original SNG Neutron Diffusion Code can assemble this extended version in operating form by replacing the SNG formulae packet with a punched-card . transcription of this listing. The FLOCO assembly packet remains unaltered. 
TABLE 2

GE-ANPD PROGRAM $S$ FORMULATION 2
INTERNAL FLOCO DEFINITIONS

048100505

048100506

048100507

048100510

^.9100511

1110512

$048220524 \quad 48110524$

048120525

04812060148110601

048110602

048110603

$048240610 \quad 48110610$

048260613

$048260630 \quad 40100630$

048260623

048260625

048260635

048120636

048260637

$048120700 \quad 48110700$

04812070148110701

048110702

048110703
04812060048110600

DR $(I) / 2$

$S R(1) / 2$

$D R / S R$

OH(I)

$Q(1)$

OV (I)/2

PH ( INLM)

$L(N)$

PH $(1, N)$

$J(I, N)$

$P(1-)$

$P(1+)$

$A(I, S)$

$Q(N H)$

$S(T H, N H)$

$\mathrm{PHI}(\mathrm{NH})$

L N / PHI

$\mathrm{D}(\mathrm{NH})$

$A(N H)$

$S G S(N H)$

$P H *(I, N)$

$J *(I, N)$

$P * F(I-)$

$P *(I+)$

CROSS SECTION MIXING

\section{SUBROUTINE 401}

$040000401 \quad$ LXA1813 $70001243 \quad$ SXD4840 CLA1550

- STAO230 SXD1841

O CLA1000 STA0225

- FMP 4000 FAD0830

0 LXD184I 20011204

LXA 1812

STA0235

LXA2110 LXA4110

CLA4000 TZE0234

20014216 CLAOB30

LXD4840 TRA4001
STA0216 STZ0830 CLA2000 STO200O
CLA 1560

CLA4000

TZE0233

20012214
STA0223SNG MXOO

STA0220SNG MX10

LRS0043SNG $M \times 20$

20011213 SNG MX30

SNG $M \times 40$

GEOME TRY

3ROUTINE 402

$040000402 \quad L X A 2815$

- FAD 1000

SUBO111

CAL 0207

SUBOI01

STAO 210

STAO213

LXA1810 STO1506

FSB 1000

STO1505 70002225

FDH1506

STO1512 20011207

CLAI5OISNG GEOO STQ1507SNG GE10 TRA400ISNG GE2O 
SUBROUTINE 403

U4 U000403 LXA1814

O LXA1810 CLA1502

- FAD0830 TPL0223
LXD 2841

STA0213

CLAO100
LDQ0 100

CLA2000

LRS0043
70011207 SUBO 107

FMP 1505
CLAO802 STAO2 17 STO1510
FDH2526 LDQ 1504 20011211
MEAN FREE PATH

STQO830SNG HIOO FMPOOOOSNG HIIO TRA4001SNG H120

\section{SUBROUTINE 404}

040000404

O FMP 1503

O FAD0830 LRSO043

U FMP 1504

O STO1511
LXA1810 STO0830 STO0 330 FMP 1000 FAD 0830 20011202
LXD 2841 CLA0816 ST 20831 FAD0831 ADDO112 TRA4001
CLA 1502

TZE0222

CLA2523 STO0831 LRSO043
STAO205

CLA2513

LXA2106

CALO231

FMP 1505
CLA2000

STAO 216 STAO231 SUBO 810 LDQ0 120
STAO226

LDQ1000

CLA 2000 20012225

TLQ0250

FREE FLIGHT SOURCE

SUBROUTINE 405

040000405

O CLA1524
CLAO817. SUBO101
TZE0206 STAO21 14
LXD1841 78211216
LDQ1563 TRA0207 STQOOOO 20011210
LDQ0100 TRA4001

\section{SUBROUTINE 406}

040000406

0 LDQ 1521

0 LDQ0837
LXA1815 ST00836

FMP 2520
30011206 STQ0837 STO0835
CLAO121

FMP2516 LDQ0837
LDQO121 STO0 833 FMPO 836
TRAO211 LDQ0837 STO0837
LXD 1842 FMP 2517 TRA4001

LDQ25275NG STOO FMP0802SNG STIO TZE0237SNG ST20 LDQ0831SNG ST30 CLAOIOOSNG ST4O SNG ST5O

SUBROUTINE 407

040000407 O STAO257 038212222

0 TRAO246

O FAD0831

O FAD0831

O FAD 0832 010011271
CLSO101 38212204 SUBO811 STAO235

LXA1810 LDQ1507 CLA0834 FAD0831 FSB 1510 LRSO043 FAD 1510 STO0830 FOH0830 TQP0264 78101222 TRA4001
CLAO101 ADD 0843 FMPO 835 FSB 1510 FMP 1000 CLAO833 LDQO 100
STO0843 CLA2524 38212210 STAO243 ADD0811 STA0264 STO0831 78202231 CLAI511 LRS0043 FMPIO00 STO0832 FAD0832 FADI511 STO0832 FSBO831 FSB1510 LRSO043 STQ1000 $38212270 \quad 20011222$

BOUNDARY CONOITIONS

LXA1822 BCOO GE $B C 10 \mathrm{GE}$

ANGULAR BASIS

CLA1522SNG SNOO STO0834SNG SN10 SNG SN2O

ANGULAR FLUX SUBOIOISNG AFOO LXA1101SNG AF1O SUBO111SNG AF 20 CLSO834SNG AF 30 ChA08335NG AF 40 FMP $1000 S N G$ AF 50 TRA0272SNG AF60 SNG AF 70

$040000410 \quad$ LXA1821 $10011203 \quad$ CLA1524 0 STQ0000
CALO207 SUBO811
STAO207
SUBO 811 CALO210
STA0207 STAO210 ADD0811 20011206
CENTER CONDITIONS

LDQOOOOSNG CCOO TRA4OOISNG CC1O 
SUBROUTINE 411

$0.30411 \quad S \times 04840$

O LXA1810 STZ0830

O FAD $1000 \quad 38202223$

O FMPO836 38214233

O STA0241 LDQ1000

0 LXD2841 38214253

0 TRA4001

\section{SUBROUTINE 412}

1000412

- LDQ1514

0 LXA2812

O STA0237

O FMP 1000

- FMP 1504

O FAD0830
LXD4842 10014204

LXA2822

30012224

FAD 1000

FMP 2515

FAD 2525
CLA2 2524

SUBO111

STO1000

FAD 0830

STO2525
LXD 2841

STAO 217

FAD 0830

20011211

STOO 830

LX04840
CLA2523

SUBO101

STO0830

LXA2822

20012237

LXA2815
TOTAL FLUX AND LEAKAGE

STA0232

STAO220

20012213

STZO 830

LRSO043

30012260
STA0233SNG TFOO CLA1DOOSNG TF1O FDHOB23SNG TF 20 CLA2524SNG TF 30 FMP0837SNG TF40 TRA4003SNG TF5O SNG TF6O
LXA1810

FMP 1512

STZ 0830

SUBO102

FADO8 33

STO1503

STO0830

\section{SUBROUTINE 413}

040000413

0 LDQ 1000

- FAD0831

O LXA1810

0 LXA1812

030012253

O CLAO 832
CLA0816 FMP 1512 STO0831 CLA1000 CLAO100 LRSO043 FDH0833
STZO 0831

FAD 0832

STZO 0833

STA0233

ST 00833

20011216

LDQ1514
STZO 832 STO0832

CLA2523 LDQO000 20012223

LXA 1810

FMP 1512
FISSION PRODUCTION AND
LDQ 1503 20011204 STAO234 FMP 1000 LRSO043 STZO 830 FAD 0833
FMP 1512

LXA 1810

STAO 240

FAD 0830

FMP 1504

ST 20833

STO0833
FAD0 831

CLAI502

CLA. 2000

STOO 830

STO1514

LDQ 1503

20011256
ELASTIC SCATTER

STO0831SNG FDOO STA0226SNG FD 10 SUBO106SNG FD2O LDQ000OSNG FD30 LDQ0830SNG FD 40 FMP 1512SNG FUSO TRA4001SNG FD60

\section{SUBROUTINE 414}

040000414

O TZEO213

O TRAO257

O FDHO 802

020011245

o TRA4001
- CLA0830
TZE0222

FAD 0837

LXA2814

FDH2526

FAD 1525

FMP 1501

STQ0836
LXA2812 STOO 837 70012240 FMP 1512 20011242

FDHO 122

FMP0 831
STZ 20837 20011210 LXA2812 FAD 0835 LXA2815 STQ0834 STO0831
CLA2513 20012205 ST 20835 STO0835 70002253

CLAO 122 TRA4001
MISCELLANEOUS AVERAGES

STAO210 LRSO0 43

CLA2523 20011231

LRS0043

FSB0 806
LXA18IOSNG AVOO FMP0802SNG AV10 STAO23ISNG AV 20 20012226 SNG AV 30 FMP150ISNG AV40 TPL4002SNG AV50 SNG AVGO
CLA0804 ST00805 LDQ0836 TRA0257 LXA2814 30002234 STQ1503 20011227 FSB0834 FDH0836 TRAO257 LDQ0802
CLAO803

CLAO816

CLA0830

TRAO260 STQ0836 FMPO 835
ST00804 TZEO221 FDHO831 30012252 LXA 1811 FAD 0830
CLAO802 CLA0831 STQ0802 CLA0831 CLA1501 FSB0831
ST00803

FSB 0830

LXA1810

FSB0 834

FDH0 836

FDHO 835
PARAMETER CHANGE

CLAO817SNG CPOO FDH0837SNG CP10 CLA1503SNG CP2O STO0836SNG CP3O STO1501SNG CP40 STQ0802SNG CP5O SNG CD 60 0100118351001183450011831

10011807 LXD 4840
1001180610011805 TRA4001
MPOO GE MP $10 \mathrm{GE}$ 
SURROUTINE 4I'5

CONVERGENCE TEST

U4U000415 CLA0806

0 TRA4002 CLAOIII

0 LXD4150 LXA1103

020011222 CLAOIOI
FAD0121

ALS0006

CLA1806

STO0127
STO0806 ADD0040

TZEO235

PSEO143
MSEO 144 STPO0 040

FSB 1805

TRA4001
PSE 0144

TNO0221

LDQ0801

PSE0166
MSEO 143

SXD4 150

LLSO000

TRA4001
TRA0211 EX00 GE TSX4417 EX10 GE TLQ0235 EX20 GE TRA0233 EX30 GE

SUBROUTINE 416

040000416

ก18111501

TS $\times 4222$

U STA0236

o ST04000
$5 \times 04841$ 18101503

TRAO216

STAO240

STQ4000
CLAO807 TMIO205 1810151458122523 SXD4140 LXA1812 CLA1523 STA0237 20014236
TRAO206

CLAO 807 LXA2101 STAO241 1001224420011225

\section{SUBROUTINE 421}

040000421 010101565

0 CLA4000

- T TSX4772 018241562 018211522
SXD4153 10101566

TZE0227 18132550 18251725 18101503
$\mathrm{TS} \times 4772$ 58122567 STA0225 18132560 18261726 18101514
10301830 35500020

SXD4 152 18101502 18221515 58122523
$14001770 \quad 18111501$ CLAO213 TS $\times 4772$ 18101504 18221516 LXD4153
ARSO022

58122000

18121563

18221517

TRA 4001
$T S \times 4222$ TMIO 0220 SXD 2226 LXA4810 LXD 4140
TS $\times 4774$ LXD4841 70001245 LDQ4000 TRA400I

DUMP

$\begin{array}{rrr}10101810 & \text { DPOO } & \mathrm{GE} \\ \text { TRA4001 } & \text { DP10 } & \mathrm{GE} \\ \text { CLA2523 } & \text { DP20 } & \mathrm{GE} \\ \text { CLA4000 } & \text { DP30 } & \mathrm{GE} \\ & \text { DP40 } & \mathrm{GE}\end{array}$

INPUT PRINT

\section{SUBROUTINE 424}

040000424

O CLA2601

- STA0222

- LDQ 1000

- FMP0836

O STO1000
SXD4840 STA0247 STAO230 FMP 2515 38214243 20011214
LXD4842 10014204 STAO250 LXA1811 CLA1000 38202225 FAD0861 FAD 1000 LXD 4840
STO0861 STO 1000 TRA 4001
LXD2841 ST 20860 30012226 20012217 LDQ0 861

\section{SUBROUTINE 425}

040000425

O STAO224

$\cap$ FMP 1000 LDQ0860

บ CLA0816

O CLA 1603
LXA 1810 SUBO101 FAD 0861 FMP 1504 ADDO 817 FDHO8O2
CLA1502 STAO220 STO0861 STO1602 ADD0814 STQ1603 20011245
STAO213 LXA2812 CLA2000 SUBO110 LDQ0000 FMP 1000 FMP 1504 LXA 1811 TRA4001
1812152618121527 IPOO GE STA0220 LXA4213 IPIO GE LXD4152 20014220 IP20 GE 1812251318242570 IP30 GE. $1822152018211521 \rrbracket P 40 \mathrm{GE}$ IP $50 \mathrm{GE}$

\section{LATTICE POINT FLUX AND CURRENT}

CLA 2600 ST20861 SUBO111 CLA0860 FMP 0837
STA0242 LXA2822

FAD 0860 ADDO111 38214250
STA0243 FJOO GE CLA2524 FJ1O GE STO0860 FJ20 GE FDH0823 FJ30 GE FAD 1000 FJ40 GE FJ50 GE

\section{LATTICE POINT FISSION PRODUCTION}

STZO 086 STA0217

FAD 0860 10011236 CLA 1602
STZ20861 STAO223 STO0860 STO1603 FDHO 802
CLA2600 FPOO GE LDQ0000 FP1O GE 20012207 FP20 GE 20021202 FP30 GE STQ1602 FP40 GE FP50 GE 
SUBROUTINE 430

$0<\quad 30430$

010011211

- CLA 1512

O LXA 1106

0 FMP0151

- LDQ 1603

- LDQ 1000

- STO1000

020011236
CLAO816

FAD 1603

FAD 0151

LDQ1836

STO1503

FMP0151

FMPO151

CLA2601

TRA4001
ADO0817 20011213

STOO151

FMPO151

LDQ 1514

STO1603

STO 1000

STAO274
TNZ4001

TZEO223

20011207

STO1836

FMPO 151

LXA2812

CLA2600

STAO276
STZO150 LRSO043 CLAO151 20011231 STO1514 38101263 STAO266 LDQ 1000
FISSION PRODUCTION NORMALIZATION

STZO151

FMP 1512

ADDO111

LXA 1811

LDQ1602

CLA2 2523

STAO270

FMPO151
LXAI610

FADO150

FDHO150

38101245

FMPO 151

STA0260

LDQ 1000

STO1000
CLA1602 NF00 GE STO0150 NF10 GE STQ0151 NF20 GE LDQ1503 NF30 GE STO1602 NF4O GE STA0262 NF5O GE FMP0151 NF60 GE 20012254 NF7O GE NF80 GE

\section{$\therefore \quad$ ROUTINE 426}

040000426

O STA0217

- FADOI50

0 LXD4 140
CLAO824

TZE0231 LXA2811 STZO150 STOO150 20014214 TRA 4001
$\operatorname{SXD4} 140$ LXA4812 LDQO 150
LXA 1824 CLA4600 FMP 1562
CLA 1610 STA0216 STO2000
STAO225 LDQ2000 20012212
SENSOR ACTIVATION

CLA1570 SAOO GE FMP 4000 SA10 GE 20011205 SA20 GE SA 30 GE

SUBROUTINE $431-435$

SPACE-ENERGY HOMOGENIZATION

$040000431 \quad$ LXA.1826 30001204 0 STZ2143 $20012210 \quad$ LXA2810

040000432

$0 \quad$ LDQ 0121 030012224

020011226

040000433

O CLAO816

- CLA 1600

- CLA1601

O CLAO151

O STZOIS3

0 STOO147

O CLAO 816

- FAD 2623

- STO2613

- CLA1600

-014331

ADO153

- FDHO122

O STQ2635

- FADO153

040000434

O ADDO101

- CLA1502

- FMP 1000

o -.ग 1512

$-12623$
SXD1130

LXA4815

LXD1131

STO0144

CLA0812

TZEO213

STA0223

STA0232

FDH4505

LXD 11.41

CLA1523

TZE0276

STO2623

LDQ0144

STA0324

FSB 4000

STO0153

STQOI51

CLAO152

FADO153

SXD4243

SUB 2726

STA0223

FAD 0152

FADO151

STQ4000
CLAO811

70004217

10011223

CLAOL 21

ADDO101

STZ2613

STAO225

CLA4000

FMPO145

CLA0816

STAO263

LDQO147

70004304

FMP 2623

STA0327

60014333

70001344

LDQO153

FDH2623

FDHO143

LXA 1812

PAX2000

LXD2141

STO0152

STO0151

70074254
TRA4001 SXD2131

SUB 1725 PAX 1000

LDQ1501 30014217

10012210 LXD1131

FDHOI44 STOO144

SUB 2726

STZ2 2623

CLA4000

FADO 152

STOO151

TZEO261

CLA4000

FMP 4512

20014247

STO2623

STAO331

FADO 147

20011320

FMPO 146

STQ2625

STQ2636

SXD1141

SXO2233

CLA2 000

70002235

70001245

20014215
PAX 1000

LXD4131

10014225

STOO152

LDQO 152

CLA1513

FADO 153

FAD 2613

CLAO816

ST 20143

CLA4000

STOO147

CLAO 147

FSBO 152

LDQO 125

60011377

SXD 2140 LXA4 110 STA0227 20012223

20011217

LXD4233
SXD 1227

FMP 1501

CLAOI0O

LXA2 826

SXD 1236

STZ0151

FSB 4000

60014236

FMPO 145

STA0256

STO0153

STO2613

TZE 0311

STZO147

FADO 143

CLA1601

FDH4505

STOO 152

FMPO 143

SXD1141

CLA2630

STZO151

CLA2523

LDQO152

LDQO151

60014260 


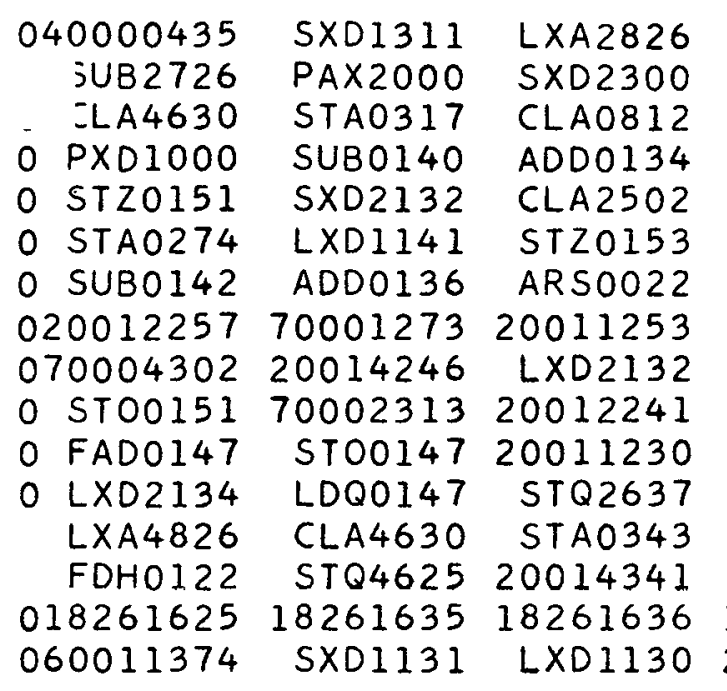

SUBROUTINE 444

$040000444 \quad S \times D 4867$ 01811160258111603 058242610

- CLA0825

O STQ0825
TSX4771

LDQ0101

LXD4867
TS $\times 4417$ TRA0221 18111501 TLQ0234 TRA4001
LXA4812

LXA4826

ADDO101

ARS0022

STAO254

SXD 1142

SUBO 106

LXD 2132

LDQO152

LXO1137

LXD4271

LXD1300

CLA2000

T $\$ \times 4772$

18261637

20011432
SXD4135

LXA1812

SUB 4726

SUBO 106

LXD4135

CLA1000

TNZ0270

LDQ 2000

FMP 2504

LXD2 134

60014330

60011337

FSB 4637

18261613

58261525

LXD4 150
SXD 2134

SXD 1141

PAX4000

TNZ0322

STZO152

STA0265

CLA2 000

FMPO 153

LRS0043

FDH 2623

SXD 4141

SXD1135

STO 4525

58262630

TS $\times 4774$

TRA4001
CLA0812

ST20147

SXD 4271

SXD1137

SXD4 136

LXA2 106

FADO 153

FAD 0152

FMP 2512

FMPO 144

LXD4140

20012205

LDQ4625

TSX4771

58262630

ADD0101 H340 GE SXD4140 H350 GE LXA1106 H360 GE LXD2131 H370 GE CLA4523 H400 GE PXD2000 H410 GE STO0153 H420 GE STO0152 H430 GE FADO151 H440 GE STO1000 H450 GE $20014217 \mathrm{H} 460 \mathrm{GE}$ LXA2107 H470 GE FMP $0144 \mathrm{H} 500 \mathrm{GE}$ $18261623 \mathrm{H} 510 \mathrm{GE}$ LXD1311 H520 GE H530 GE

OUTPUT PRINT

CLA0824 TSX4771 18111512 TRAO241
TN20213 18111501 18122600 STO0 857
TS $\times 4771$ 18111512 58122601 STQ0825

\section{SUBROUTINE 445}

040000445

O LXA2812

O STA0224

- CLS0800

O TRA4001
LDQ0802 STQ0032 CLA2600 STA0221 LDQ1000 STQ1000 ST00800 LXAII04
LXA1811 CLA2700 LDQ 1000 STZ1807
LDQ 1602 STAO222 STQ 1000 20011233
STQ1702

CLA2601 20012211 CLSO121

SUBROUTINE 450-45I

040000450 020011205

O STA0232

. O STZO150 TZEO254 LDQ4636 o SXD1142 020014271

O LXA2110

- CLA4000 020011303
MSE0 142 35500020 STAO242

LXA4812

LXA4812

FMPO 150

LXA4812

20011262

LXA1812

STA0314 20012301
PSEO142 CLA0211 STA0262 CLA4000 CLA4000 STO2000 CLA 1000 CLA4000 LXA4101 STA0316 LXD1141
SXD4 140 ARSO0 22 STA0264 STAO 234 STAO 245 20014242 STA0266 STA0 274 SXD4304 LDQ2 2000 20011216
LXA1812 STAO 216 STAO 272 CLA2 2000 STAO 252 SXD1141 CLA4000 STZ2000 70001321 CLA2 2000
LDQ1527 LXA1211 STA0305 FADO 150 CLA2 000 LXA2 105 STA0267 20014272 CLA 1000 STO2000
LDQ1603

STA0223 20011204 STO0807
STQ1636 CLA1000 STA0310 STOO15O FDHO150 LXA 812 LDQ2000 LXO1142 STA0 313 STQ2000
18111512 18111603 TSX4431 LDQ0857

TSX4416 LDQ0857

FIELD STORAGE STQ1703 FSOO GE CLA2701 FS10 GE STZ0040 FS20 GE STZ0127 FS30 GE FS40 GE

ADJOINT

STZ1527 A000 GE TZE0323 AO10 GE LXA2110 A020 GE 20014232 A030 GE STQ4527 A040 GE 60011300 A050 GE STQ2000 A060 GE 20012257 A070 GE STA0315 A100 GE 10014320 Al10 GE A120 GE 


\begin{tabular}{|c|c|c|c|c|}
\hline 040000451 & LXA1812 & LXA2101 & SX02204 & 70 \\
\hline - CLA 2563 & STO1563 & STQ2563 & CLAO 816 & \\
\hline $\mathrm{CL}$ & & & & {[} \\
\hline 0200 & & & 2 & $C L$ \\
\hline $0 \quad L D C$ & & & ST & 2001 \\
\hline $0 \mathrm{ST}$ & ST & 66 & 267 & LDC \\
\hline & 100 & 200 & 326 & 700 \\
\hline$-D$ & & & & SUE \\
\hline 0 & & & & \\
\hline
\end{tabular}

SUBROUTINE 420

040000420

O TRA0212

O LXA2821

o TSX4406

o TRAO243

O TSX44i2

o TSX4444

O LXA4101

- TRA4000

$\stackrel{+}{0}$ TRA 0420

TS $\times 4421$ TSX4421 SXD2842 TSX4407 20012230 $T S \times 477158222524$ TSX4413 TSX4414 CLAO127 TZE0267 CLA0301 STO400O
CLA0807 LXA2812 TSX4406 MSEO 143 TSX4411 TS $\times 4415$ CLA0807 PXD0000
TMI0206 SXD 2841

TS $\times 4407$

TRAO 243

LXD 2842

TRAO21 2

TNZO267

MSE 0003 $\begin{array}{rr}\text { LDQ1526 } & \text { CLA2526 } \\ \text { CLA0817 } & \text { TZEO223 } \\ \text { CLA1513 } & \text { STAO234 } \\ \text { CLA4000 } & \text { STO4000 } \\ \text { STAO251 } & \text { STAO253 } \\ \text { LXA4824 } & 70004271 \\ \text { CLA2000 } & \text { STO1000 } \\ \text { LXA2102 } & \text { CLAO812 } \\ \text { STO1726 } & 10012307\end{array}$
STO1526 A200 GE LDQ1563 A210 GE STA0236 A220 GE STQ4000 A230 GE LXA4810 A240 GE CLA4570 A250 GE STQ2000 A260 GE SUB 1726 A270 GE SXD2310 A300 GE A3 $10 \mathrm{GE}$
TRAO212 TSX4403 20012226

PSEO143 20012221

TSX4425 TSX4445 PSE 0006
TS $\times 4450$ T S $\times 4404$ 38212223

TSX4424 LXD 2841 TS $\times 4430$ TRA0206 STOO 150

\section{FLOW CODE}

$\begin{array}{rll}\text { PSE0165 } & F C O 0 & G E \\ \text { TSX4405 } & F C 10 & G E \\ \text { TSX4410 } & F C 20 & G E \\ \text { PSE0165 } & F C 30 & G E \\ 20012214 & F C 40 & G E \\ \text { TSX4426 } & F C 50 & G E \\ \text { STZ0127 } & F C 60 & G E \\ \text { LDQ0150 } & F C 70 & G E \\ & \text { FC1.0OGE }\end{array}$

TRANSFER BL.ANK BLANK
PRINT S PUNCH F DUNCH F* 


\section{Nuclear Data}

\subsection{ANISOTROPIC SCATTER-TRANSFER CROSS SECTIONS}

For elastic isotropic scattering in center-of-mass coordinates, with neglect of molecular binding and thermal agitation of nuclei, the scatter-transfer cross section for a nucleus having a mass $A$ times that of a neutron is given by*

$$
\sigma_{A S}\left(p \mid p^{\prime}, \underline{\Omega} \cdot \underline{\Omega}^{\prime}\right)=\frac{(A+1)^{2}}{2 A} \frac{\sigma_{A s o}(p)}{p^{2} p^{\prime}} \delta\left[\underline{\Omega} \cdot \underline{\Omega^{\prime}}-\left(\frac{A+1}{2} \frac{p^{\prime}}{p}-\frac{A-1}{2} \frac{p}{p^{\prime}}\right)\right] .
$$

The momenta $p \mid p^{\prime}$ are arranged in chronological order ( $p$ scatters to $\left.p^{\prime}\right)$.

Constructing the scatter-transfer $L$-th Legendre moment, by multiplying equation (3.1.1) by $P_{L}\left(\Omega \cdot \Omega^{\prime}\right) d\left(\underline{\Omega} \cdot \Omega^{\prime}\right)$ and integrating over all scattering angles, yields

$$
\begin{gathered}
\sigma_{A S L}\left(p \mid p^{\prime}\right)=\frac{(A+1)^{2}}{2 A} \frac{\sigma_{A S O}(p)}{p^{2} p^{\prime}} P_{L}\left(\frac{A+1}{2} \frac{p^{\prime}}{p}-\frac{A-1}{2} \frac{p}{p^{\prime}}\right) \\
\times\left[\begin{array}{l}
1, p \frac{A-1}{A+1}<p^{\prime}<p \\
0, \text { otherwise }
\end{array}\right]
\end{gathered}
$$

Constructing a digital representation for this transfer moment, by incrementally averaging over an epithermal entrance flux having a uniform distribution in lethargy $u$ and incrementally integrating over the corresponding exit adjoint (which has a uniform distribution in momentum space) normalized to unity, yields

$$
\begin{gathered}
\sigma_{A S L n n^{\prime}}=\int \frac{(A+1)^{2}}{2 A} \frac{\sigma_{A s 0}(p)}{p^{2} p^{\prime}} P_{L}\left(\frac{A+1}{2} \frac{p^{\prime}}{p}-\frac{A-1}{2} \frac{p}{p^{\prime}}\right) \\
\times\left[\begin{array}{l}
1, p \frac{A-1}{A+1}<p^{\prime}<p \\
0, \text { otherwise }
\end{array}\right] \frac{d_{n} u}{\Lambda_{n} u} p^{\prime 2} d_{n^{\prime}} p^{\prime},
\end{gathered}
$$

where $d u=-2(d p) / p, \Lambda_{n} u=u_{n}-u_{n-1}$, and $d_{n}$ signifies a differential lying within the increment $n$.

A digital representation of the the rmal cross section is obtained by averaging the transfer moment (equation 3.1.2) over a Maxwellian entrance flux and integrating over the Maxwellian exit adjoint (which has a uniform distribution in momentum space) normalized to unity.

The integration for the isotropic component was worked out in GE-ANPD APEX-394. ${ }^{\dagger}$ Performing the integration for the first Legendre moment, with $\sigma_{A S}(p) d_{n} u$ approximated by $\bar{\sigma}_{A s n} d_{n} u$ within each epithermal increment, yields:

For $n>n^{\prime}, \bar{\sigma}_{A S 1 n n^{\prime}}=0$.

*Marshak, R. E., "Theory of Slowing Down of Neutrons by Elastic Collision with Atomic Nuclei,"

Rev. Mod. Phys. 19, 185-238 (1947).

†Duane, op. cit., equations 2.356.3-2.356.6. 
For epithermal $n=n^{\prime}$, with $\xi_{A 1}=\frac{5}{3}-A^{2}+\frac{(A+2)(A-1)^{2}}{4} \ln \left(\frac{A+1}{A-1}\right)^{2}$,

and with $\bar{\sigma}_{A s 1 n}=(2 / 3 A) \bar{\sigma}_{A s o n}$,

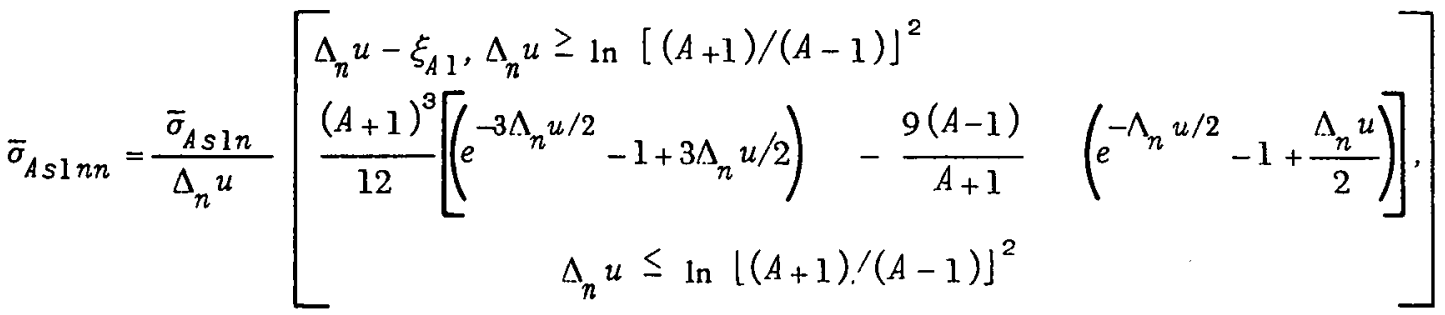

For thermal $N=N^{\prime}, \bar{\sigma}_{A S 1 N N^{\prime}}=\bar{\sigma}_{A S 1 N^{\circ}}$.

For $n<n^{\prime}$, with

$F_{A S 1}(\Delta u)=\left[\xi_{A 1}-\Delta u+\frac{(A+1)^{3}}{12}\left\{\left(e^{-3 \Delta u / 2}-1+3 \Delta u / 2\right)-\frac{9(A-1)}{A+1}\left(e^{-\Delta u / 2}-1+\Delta u / 2\right)\right\}\right]$
$\times\left[\begin{array}{l}1,0 \leq \Delta u \leq \ln [(A+1) /(A-1)]^{2} \\ 0, \text { otherwise }\end{array}\right]$,
$\bar{\sigma}_{A S 1 n n^{\prime}}=\frac{\bar{\sigma}_{A S 1 n}}{\Lambda_{n} u}\left[F_{A S 1}\left(u_{n^{\prime}-1}-u_{n}\right)-F_{A S 1}\left(u_{n^{\prime}-1}-u_{n-1}\right)-F_{A S 1}\left(u_{n^{\prime}}-u_{n}\right)+F_{A s 1}\left(u_{n},-u_{n-1}\right)\right]$.

From the definitional identity,

$$
\sigma_{A s L}(p)=\int_{0}^{\infty} \sigma_{A s L}\left(p \mid p^{\prime}\right) p^{\prime 2} d p^{\prime},
$$

it follows that these digitalized scatter-transfer moments satisfy the identity,

$$
\bar{\sigma}_{A S L n}=\sum_{n^{\prime}=1}^{n^{\prime}:=N} \bar{\sigma}_{A s L n n^{\prime}} .
$$

These formulas simplify extensively for heavy elements and for hydrogen. The calculational complexity is at its worst for deuterium.

Numerical calculation of these scatter-transfer first Legendre moments has been incorporated into an IBM 704 electronic data processing machine program.* A numerical compilation including these scatter-transfer first moments for fifty elements of interest in reactor design work has already been published; $^{\dagger}$ but meeting immediate development needs precluded providing the supporting first-moment analysis work in APEX-394, where it logically belongs.

The following section extends this neutron transfer cross section compilation to include compounds and alloys of special interest in reactor development work.

*GE-ANPD program SIGMA.

'Duane, op. cit.. pp. 20-93. 
There is urgent engineering need for assembling a similar compilation of photon cross sections, processed for use with transport theory. An initial compilation having a fairly wide range of usefulness seems obtainable from the Klein-Nishina theory of free electron effects supplemented by a semi-empirical representation for bound electron effects and for pair production. 


\title{
Cross Sections for Reactor Analysis
}

\author{
Volume Three \\ Neutron Transfer \\ Preliminnry Data
}




\begin{tabular}{|c|c|c|c|c|c|}
\hline$\underset{N}{\text { LEVEL }}$ & $\begin{array}{l}\text { ENERGY } \\
E(E V)\end{array}$ & & $\begin{array}{c}\text { LETHARGY } \\
U=L N(I O M E V / E)\end{array}$ & $\begin{array}{l}1 / V E L O C I T Y \\
1 / V(S E C / C M)\end{array}$ & $\begin{array}{l}\text { BAND } \\
\quad N\end{array}$ \\
\hline 0 & + INF INITY & & -INFINITY & 10 & 1 \\
\hline 1 & $6.0653065 E$ & 06 & $5.0000000 E-01$ & $3.3351009 E-10$ & 2 \\
\hline 2 & $3.6787944 E$ & 06 & $1.0000000 E 00$ & $4.2823544 E-10$ & 3 \\
\hline 3 & $2.2313016 E$ & 06 & $1.5000000 E 00$ & $5.4986518 E-10$ & 4 \\
\hline 4 & $1 \cdot 3533528 E$ & 06 & $2.0000000 E 00$ & $7.0604085 E-10$ & 5 \\
\hline 5 & $8.2084998 E$ & 05 & $2.5000000 E 00$ & $9.0657440 E-10$ & 6 \\
\hline 6 & $4.9787068 E$ & 05 & $3.0000000 E 00$ & $1.1640646 \mathrm{E}-09$ & 7 \\
\hline 7 & $3.0197383 E$ & 05 & $3.5000000 E 00$ & $1.4946885 E-09$ & 8 \\
\hline 8 & $1.8315639 \mathrm{E}$ & 05 & $4.0000000 E 00$ & $2.9026958 E-09$ & $\oint$ \\
\hline 9 & $2.4787522 E$ & 04 & $6.0000000 E 00$ & $7.8903449 E-09$ & 10 \\
\hline 10 & $3.3546263 E$ & 03 & $8.0000000 E 00$ & $2 \cdot 1448182 E-08$ & 11 \\
\hline 11 & $4.5399929 E$ & 02 & $1.0000000 E 01$ & $5.8302204 E-08$ & 12 \\
\hline 12 & $6.1442124 E$ & 01 & $1.2000000 E 01$ & $1.5848182 E-07$ & 13 \\
\hline 13 & $8.3152871 E$ & 00 & $1.4000000 E 01$ & $3.7339754 E-07$ & 14 \\
\hline 14 & $1.8553914 E$ & 00 & $1.5500000 E \quad 01$ & $6.8863408 E-07$ & 15 \\
\hline 15 & $6.8256034 E-$ & -01 & $1.6500000 E 01$ & $1.1353657 E-06$ & 16 \\
\hline 16 & $2.5109992 E-$ & -01 & $1.7500000 E 01$ & $1.8719016 E-06$ & 17 \\
\hline 17 & $9.2374495 E-$ & -02 & $1.8500000 E 01$ & $3.1326930 E=06$ & 18 \\
\hline 18 & $3.2162206 \mathrm{E}=$ & .02 & 1.9555059E 01 & $4.0313012 E-06$ & 19 \\
\hline 19 & 0 & & + INF IN ITY & & \\
\hline
\end{tabular}




\begin{tabular}{|c|c|c|c|c|c|}
\hline ATOM & FORMULA & MASS FRACIION & ATOM & FORMULA & MASS FRACTION \\
\hline $\mathrm{H}$ & $2.0 \% 00000 E+100$ & $1.0000000 \mathrm{~F}+00$ & & & \\
\hline & \multirow[t]{2}{*}{$\begin{array}{c}\text { FORMULA } \\
\text { F }\end{array}$} & $\begin{array}{c}\text { DENSITY } \\
\text { RHO }(G / C C)\end{array}$ & $\begin{array}{l}\text { FUEL } \\
\text { RHOF }\end{array}$ & $\begin{array}{l}\text { DENSITY } \\
(G / C C)\end{array}$ & $\begin{array}{l}\text { TEMPERATURE } \\
T(\text { DEG K) }\end{array}$ \\
\hline & & $8.3803942 E-05$ & 0. & & $2.9316000 E 02101$ \\
\hline
\end{tabular}

PROSABILITY/PATH (/CM)

\begin{tabular}{|c|c|c|c|c|c|}
\hline \multirow{2}{*}{$\begin{array}{l}\text { COLLIDE } \\
\text { SG(N!) }\end{array}$} & \multirow{2}{*}{$\begin{array}{l}\text { MUL TIPLY } \\
\text { NUSGF }(N)\end{array}$} & \multicolumn{4}{|c|}{ SCATTER } \\
\hline & & $\operatorname{SGSO}(N, N)$ & $\operatorname{SGSI}(N, N)$ & $S G S O(N, N+1)$ & $\operatorname{SGS} 1(N \cdot N+1)$ \\
\hline $\begin{array}{l}5.7603 E-05 \\
8.5152 E-05 \\
1.2147 E-04 \\
1.6530 E-04 \\
2.1538 E-04\end{array}$ & $\begin{array}{l}0 . \\
0 . \\
0 . \\
0 . \\
0 .\end{array}$ & $\begin{array}{l}1.2273 E-05 \\
1.8142 E-05 \\
2.5880 E-05 \\
3.5218 E-05 \\
4.5890 E-05\end{array}$ & $\begin{array}{l}1.1387 E-05 \\
1.6832 E-05 \\
2.4010 E-05 \\
3.2674 E-05 \\
4.2574 E-05\end{array}$ & $\begin{array}{l}1.7836 E-05 \\
2.6366 E-05 \\
3.7610 E-05 \\
5.1181 E-05 \\
6.6590 E-05\end{array}$ & $\begin{array}{l}1.4259 E-05 \\
2.1078 E-05 \\
3.0066 E-05 \\
4.0912 E-05 \\
5.3309 E-05\end{array}$ \\
\hline $\begin{array}{l}2.7549 E-04 \\
3.5814 E-04 \\
4.5331 E-04 \\
6.9875 E-04 \\
9.3920 E-04\end{array}$ & $\begin{array}{l}0 . \\
0 . \\
0 . \\
0 .\end{array}$ & $\begin{array}{l}5.8696 E-05 \\
7.6305 E-05 \\
9.0582 E-05 \\
3.9665 E-04 \\
5.3313 E-04\end{array}$ & $\begin{array}{l}5.4456 E-05 \\
7.0793 E-05 \\
8.9605 E-05 \\
3.1834 E-04 \\
4.2797 E-04\end{array}$ & $\begin{array}{l}8.5301 E-05 \\
1.1089 E-04 \\
3.0845 E-04 \\
2.6120 E-04 \\
3.5108 E-04\end{array}$ & $\begin{array}{l}6.8186 E-05 \\
8.8644 E-05 \\
2.0212 E-04 \\
1.4033 E-04 \\
1.8881 E-04\end{array}$ \\
\hline $\begin{array}{l}9.9184 E-04 \\
1.0020 E-03 \\
1.0099 E-03 \\
1.0331 E-03 \\
1.0544 E-03\end{array}$ & $\begin{array}{l}i . \\
0 . \\
0 . \\
0 .\end{array}$ & $\begin{array}{l}5.6299 E-04 \\
5.6868 E-04 \\
5.7294 E-04 \\
4.9744 E-04 \\
3.8696 E-04\end{array}$ & $\begin{array}{l}4.5193 E-04 \\
4.5641 E-04 \\
4.6014 E-04 \\
4.1522 E-04 \\
3.3786 E-04\end{array}$ & $\begin{array}{l}3.7074 E-04 \\
3.7449 E-04 \\
3.3899 E-04 \\
3.3781 E-04 \\
4.2030 E-04\end{array}$ & $\begin{array}{l}1.9937 E-04 \\
2.0120 E-04 \\
1.9133 E-04 \\
2.1399 E-04 \\
2.8149 E-04\end{array}$ \\
\hline $\begin{array}{l}1.1211 E-03 \\
1.2365 E-03 \\
1.4752 E-03 \\
1.6926 E-03\end{array}$ & $\begin{array}{l}0 \\
0 \\
0\end{array}$ & $\begin{array}{l}4.1120 E-04 \\
4.5403 E-04 \\
5.6570 E-04 \\
1.6780 E-33\end{array}$ & $\begin{array}{l}3.5606 E-04 \\
3.8834 E-04 \\
4.7159 E-04 \\
8.6153 E-04\end{array}$ & $\begin{array}{l}4.4663 E-04 \\
5.0853 E-04 \\
8.9815 E-04\end{array}$ & $\begin{array}{l}2.8915 E-04 \\
3.0913 E-04 \\
3.1888 E-04\end{array}$ \\
\hline
\end{tabular}


013

$1.0818 E-0$

$1.1836 E-06$ C 115

$-5.4934 E-11$ 025

$9.6994 E-1.6$ 0211

$5.2804 E-08$ C. 217

0.38

5.0900E-06 0314

$3.1856 E-10$ 046

$3.1 \cup 43 E-05$

0412

$3.7754 E-08$

0418

0.511

5.9891E-07

$0 \quad 5 \quad 17$

$2.5676 E-10$ O 611

I. $2631 E-06$ 0617

$6.5682 E-11$

C 712

$3.6639 E-07$

0718

$8.5386 E-11$

C. 814

$1.2645 \equiv-03$ ก 911

$3.5356 .0-0=$

$0 \quad 917$

1. $95755-09$

01015

$1.4199 \mathrm{E}-\mathrm{O} i$

01114

$6.1009 E-C 6$

O 1214

4. 5535E-0j C 13 i5

$6.1545 E-05$

C 1417

4.573.8E-35

01618

$1.5943 E-C 4$ $\begin{array}{lll}0 & 1 & 4\end{array}$

$6.5614 E-i 6$

O 110

$1.5018 E-07$

0116

0 .

026

$5.8830 \varepsilon-06$

0212

$7.1665 E-09$

0218

0 .

039

$6.7843 E-06$

0315

$5 \cdot 7 \subseteq 195-11$ 047

1.8828E- 55

0413

$5.1626 E-\cup 9$

0419

0.

$0 \quad 512$

$8.1109 E-38$

.0518

$-2.0540 E-10$ 0612

1.7090E-07

$0 \quad 618$

C 713

4. $9695 E-j 8$

0719

2

C . ก โ

?.1615E-09

C 912

$4.7842 \mathrm{E}-0 \mathrm{G}$

$091 \%$

$7.06025-10$

01016

$5.2256 \Xi-08$

C 1115

$1.1076 E-06$

C. 1215

$0.2672 E-06$ 0.1316

2.2641E-¿5

01418

$1.7343 E-.5$

$0 \quad 1619$

$8.9717 E-.5$ $\begin{array}{lll}0 & 1 & 5\end{array}$

$3.9797 E-06$

C 111

2.1692E-CS

$\begin{array}{lll}0 & 1 & 17\end{array}$

0.

027

$3.5682 E-06$

O 213

$9.9478 E-10$

C 219

0.310

9.1819E-07

C 316

-0 .

048

$1.1420 E-05$

0414

$5.5173 E-10$

C 57

4. C45OE-C5

0513

1. $9938 \mathrm{E}-08$

O 519

3.

0613

$2.2989 E-C 8$

C 619

0. 714

$5.8916 E-09$

C 810

4.1744E-CE

0 \& 16

$7.5653 E-13$

$0 \bigcirc 13$

$6.4738 E-07$

9919

$3.3318 \Xi-10$

i) 1017

$1.9201 E-08$

01116

$4.0744 E-07$

01216

3.0414E-Ć́

- 1317

$8.3292 E-06$

C 1419

$9.2452 E-36$

6. 1.719

$2.6712 E-04$ c 16

2.4138E-06

C 112

2.9252E-09

0118

0 .

) 2.8

2. $1643 E-06$

C 214

8.1206E-11

035

2. $2812 E-05$

O 311

$1.2425 E-07$

O 317

0 .

049

$1.5221 E-05$

0415

$1.1823 \mathrm{E}-10$

058

$2.4534 E-05$

$0 \quad 514$

$1.3351 E-09$

068

$5.1738 E-C 5$

$\therefore 614$

2. $9557 E-09$

ง 79

1. $4780 E-04$

O 715

1. 1954 E-0?

0811

$5.64945-06$

C 817

$3.24 ? 3 E-10$

C 914

$7.8757 E-0.8$

C 101 .

4. $7514 E-05$

C 1018

7.2772 E-09

01117

1. 4994 E-07

$012 \quad 17$

1.1189E-0ó

01318

$3.1597 E-06$

01517

$1.5462 E-04$
017

$1.4640 E-06$

0113

$3.9827 E-10$

C 119

0.

029

2.8847E-06

0215

4. $0603 E-11$

036

$1.3836 E-05$

0312

$1.5854 E-08$

- 318

0.

0410

2.0600E-06

0416

$1.9704 E-10$

059

$3.2701 E-05$

0515

$2.5676 E-10$

069

$6.896 C E-05$

0615

$5.2545 E-10$

O 710

$2.0003 E-05$

0716

$4.2693 E-10$

0812

$7.6448 E-07$

0 ? 18

$5.4038 E-10-3.2423 E-10$

C 915

$1.4327 E-08$

01013

$6.4303 E-06$

01019

3.5267E-09

01118

$5.6926 E-08$

01218

4. $2430 \bar{E}-07$

01319

$1.6858 E-06$

01518

$5.8655 E-05$

$\begin{array}{lll}0 & 1 & 8\end{array}$

$8.8799 E-07$

0114

$9.6134 E-11$

024

$1.5992 E-05$
0

$3.9039 E-07$

0216

0.

$\begin{array}{lll}0 & 3\end{array}$

9.3920E-06

0313

2. 200SE-09

0319

0.

0411

$2.7874 E-07$

0417

$-1.1823 E-10$

0510

4. $4255 E-06$

O 516

$5.1351 E-11$

0610

$9.3327 E-06$

0616

$1.9704 E-10$

C 711

2.7072E-00

0717

0813 
113

$6.7392 E-06$

119

$1.4071 E-07$

1115

$2.4231 E-10$

125

4.7097E-06

1211

2.5200E-09

1217

0 .

138

$1.5031 E-06$ 1314

$9.6612 E-11$

146

1.9332E-05

1412

$1.6037 E-09$

1418

0.

1511

$2.9991 E-08$

1517

$-1.3916 E-10$

1611

7.5954E-08

1617

$4.3816 E-11$

1712

$1.4697 E-08$

1718

$2.2786 E-10$

1814

1.0815E-09

1911

$7.0441 E-06$

1917

$3.6118 E-10$

11015

$9.2487 E-09$

11114

$5.2166 E-07$

11214

$9.4984 E-06$

11315

$1.7758 E-05$

$114 \quad 17$

$1.1419 E-05$

11618

$5.8391 E-05$ $\begin{array}{llllll}1 & 1 & 4 & 1 & 1 & 5\end{array}$

$3.1863 \mathrm{E}-\mathrm{C} 6$

1110

$8.9364 E-09$

1116

0 .

126

$2.2279 E-06$

1212

$6.2322 E-1 C$

1218

139

$1.2886 E-C 6$

1315

$3.8645 E-11$

147

$9.1372 E-06$

1413

$3.9436 E-10$

1419

0.

1512

$3.1002 E-09$

1518

$2.4193 \mathrm{E}-10$

1612

$6.3094 E-C 9$

1618

$1.3145 \mathrm{E}-10$

1713

$2.4779 E-00$

1719

0.

1815

$2.8840 E-10$

1912

$3.7175 E-C 7$

1918

$2.5005 E-10$

11016

$4.8205 E-09$

11115

$6.2761 E-08$

$\begin{array}{lll}1 & 12 & 15\end{array}$

$8.9020 E-07$

11316

4.0894E-06

I 1418

$2.9634 E-06$

$\begin{array}{lll}1 & 16 & 19\end{array}$

$2.6499 E-06$

1310

1316

1414

157

1513

1519

0.

1613

1519

0. 714

1810

1816

1913

1919

11017 11116

11216

11419

11719
116

$7.1381 E-07$

1112

$3.2996 E-10$

1118

0.

128

5.0022E-07

1214

$5.4193 E-11$

$1.4208 E-05$

1311

$5.3330 E-09$

$6.9251 E-08$

$9.6612 E-11$

148

$4.3202 E-06$

I. $8403 E-10$

$2.5190 E-05$

$6.8512 \mathrm{E}-10$

$1.3145 E-09$

$5.6964 E-10$

$1.0119 E-05$

$1.4420 E-10$

$2.6255 E-08$

4. $7232 E-10$

$2.7279 \mathrm{E}-09$

$2.1782 E-C 8$

$2.0942 E-07$

11317.

$9.8956 E-07$

$1.4635 E-06$

2. $8820 E-05$

0.

145

3.6894 E-06

1415

158

1817

$2.16305-10$

1914

$3.5702 E-09$

11012

9.5577E-06

11018

$1.6816 E-09$

11117

9.5492E-09

11217

$5.3276 E-08$

11318

$2.7508 E-C 7$

11517
135

$\begin{array}{llllll}1 & 1 & 7 & 1 & 1 & 8\end{array}$

$3.3856 E-07 \quad 1.6100 E-07$

$1113 \quad 1114$

$1.0999 E-10-1.5066 E-10$

1119

0.

129

4. $3228 E-07$

1215

$9.4838 E-11$

136

$6.7160 E-06$

1312

$9.4679 E-10$

1318

0.

1410

$1.9104 E-07$

1416

$2.6291 E-11-1.7417 E-10$

159

$1.0150 E-05$

1515

6. $8512 E-11$

169

$2.7445 E-05$

1515

1. $7526 E-10$

1710

$3.7924 E-06$

1716

$5.6964 \mathrm{E}-11$

1812

$3.3671 E-08$

1818

$-4.9118 E-10$

1915

$1.0280 \mathrm{E}-09$

11013

$5.3372 E-07$

11019

$3.3258 E-09$

11118

$5.2284 E-09$

11218

$1.6272 E-C 8$

11319

$1.6610 E-07$

11518

$1.3945 E-05$
$9.9618 E-06$

1210

$2.4997 E-08$

1216

0.

137

$3 \cdot 1761 E-06$

1313

$2.8983 E-10$

1319

0.

1411

$1.2251 E-08$

1417

$3.0563 E-10$

1510

5.1691E-07

1516

$6.8512 E-11$

1610

$1.3853 E-06$

1616

4. $3816 E-11$

1711

2.0155E-07

1717

$5.6964 E-11$

1813

4. $4342 E-09$

1819

$7.7959 E-10$

1916

$6.1123 E-10$

11014

$4 \cdot 2450 E-08$

11113

$1.0088 E-05$

11119

$7.7341 E-09$

11219

$1.2209 E-08$

11416

4.8670E-05

11519

$3.0842 E-06$ 


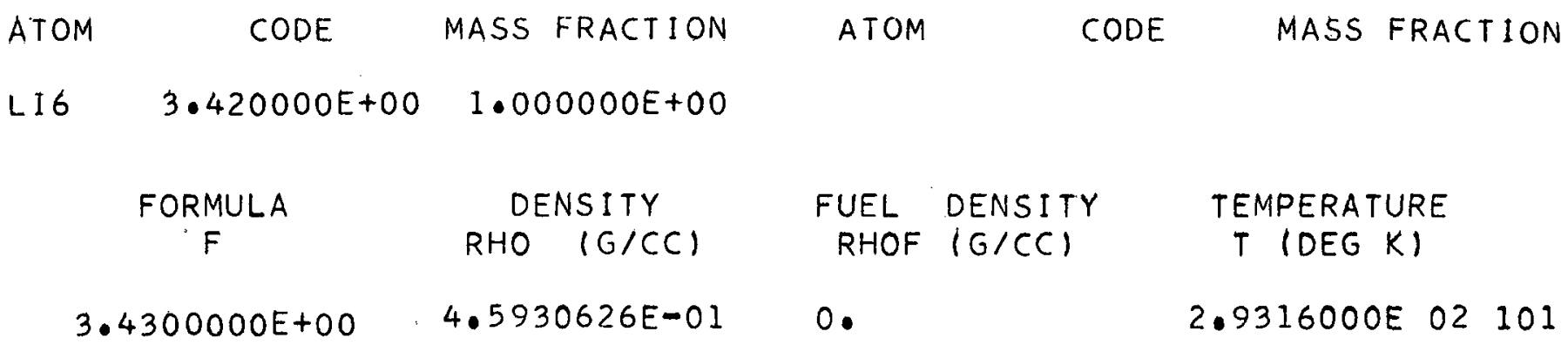

PROBABILITY/PATH (/CM)

\begin{tabular}{|c|c|c|c|c|c|}
\hline \multirow{2}{*}{$\begin{array}{c}\text { COLLIDE } \\
\text { SG(N) }\end{array}$} & MULTIPLY & \multicolumn{4}{|c|}{ SCATTER } \\
\hline & NUSGF $(\bar{N})$ & $\operatorname{SGSO}(N, N)$ & $\operatorname{SGSI}(N, N)$ & SGSO $(N, N+1)$ & $\operatorname{SGS} 1(N, N+1)$ \\
\hline $\begin{array}{l}8.6690 E-02 \\
9.4278 E-02 \\
8.6000 E-02 \\
6.4615 E-02 \\
6.1856 E-02\end{array}$ & $\begin{array}{l}0 . \\
0 . \\
0 . \\
0 . \\
0 .\end{array}$ & $\begin{array}{l}3.6548 E-02 \\
3.8839 E-02 \\
3.3660 E-02 \\
2.3005 E-02 \\
2.1411 E-02\end{array}$ & $\begin{array}{l}1.9967 E-02 \\
2.1218 E-02 \\
1.8389 E-02 \\
1.2568 E-02 \\
1.1697 E-02\end{array}$ & $\begin{array}{l}4.4937 E-02 \\
4.7753 E-02 \\
4.1386 E-02 \\
2.8284 E-02 \\
2.6325 E-02\end{array}$ & $\begin{array}{l}-8.1515 E-03 \\
-8.6624 E-03 \\
-7.5074 E-03 \\
-5.1308 E-03 \\
-4.7754 E-03\end{array}$ \\
\hline $\begin{array}{l}8.2321 E-02 \\
2.2052 E-01 \\
2.2834 E-01 \\
8.0021 E-02 \\
1.3797 E-01\end{array}$ & $\begin{array}{l}0 . \\
0 . \\
0 . \\
0 . \\
0 .\end{array}$ & $\begin{array}{l}2.7884 E-02 \\
6.0051 E-02 \\
5.4773 E-02 \\
2.3445 E-02 \\
7.8151 E-03\end{array}$ & $\begin{array}{l}1.5233 E-02 \\
3.2806 E-02 \\
2.9923 E-02 \\
4.1404 E-03 \\
1.3801 E-03\end{array}$ & $\begin{array}{l}3.4284 E-02 \\
7.3833 E-02 \\
7.1698 E-02 \\
4.1483 E-03 \\
1.3828 E-03\end{array}$ & $\begin{array}{l}-6.2191 E-03 \\
-1.3393 E-02 \\
-1.5783 E-02 \\
-1.0555 E-03 \\
-3.5182 E-04\end{array}$ \\
\hline 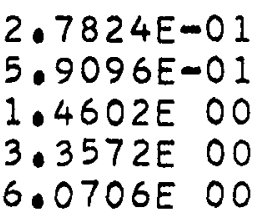 & $\begin{array}{l}0 . \\
0 . \\
0 . \\
0 . \\
0 .\end{array}$ & $\begin{array}{l}2.3445 E-02 \\
8.7920 E-02 \\
1.8561 E-01 \\
2.2063 E-01 \\
1.9297 E-01\end{array}$ & $\begin{array}{l}4.1404 E-03 \\
1.5527 E-02 \\
3.2778 E-02 \\
4.4923 E-02 \\
5.1959 E-02\end{array}$ & $\begin{array}{l}4.1483 E-03 \\
1.5556 E-02 \\
3.2841 E-02 \\
5.5311 E-02 \\
8.2966 E-02\end{array}$ & $\begin{array}{l}-1.0555 E-03 \\
-3.9580 E-03 \\
-8.3558 E-03 \\
-1.4073 E-02 \\
-2.1110 E-02\end{array}$ \\
\hline 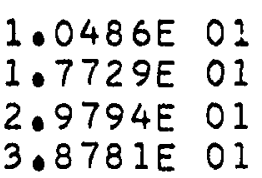 & $\begin{array}{l}0 \\
0 . \\
0 \\
0\end{array}$ & $\begin{array}{l}1.9297 E-01 \\
1.9297 E-01 \\
1.9730 E-01 \\
2.7594 E-01\end{array}$ & $\begin{array}{l}5.1959 E-02 \\
5.1959 E-02 \\
5.0858 E-02 \\
3.0850 E-02\end{array}$ & $\begin{array}{l}8.2966 E-02 \\
8.2966 E-02 \\
7.8636 E-02\end{array}$ & $\begin{array}{l}-2.1110 E-02 \\
-2.1110 E-02 \\
-2.0008 E-02\end{array}$ \\
\hline
\end{tabular}




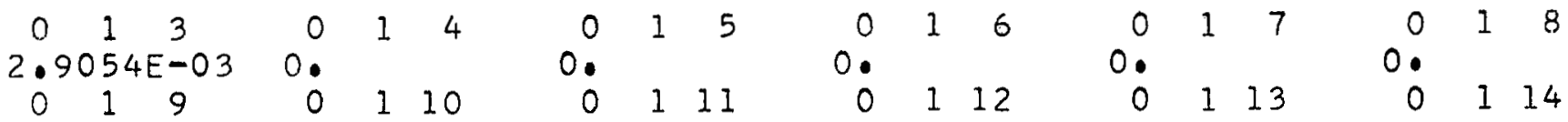
$0 . \quad 0$.

$0115 \quad 0 \quad 116$

$\begin{array}{rrrrrr}0 . & & 0 . & & \\ 0 & 2 & 5 & 0 & 2 & 6\end{array}$

$\begin{array}{rrrrrr}0 . & & 0 . & & \\ 0 & 2 & 11 & 0 & 2 & 12\end{array}$

0.00

$\begin{array}{rrrr}0 & 2 & 17 & 0 \\ 0 . & & & 0 .\end{array}$

$\begin{array}{llllll}0 & 3 & 8 & 0 & 3 & 9\end{array}$

$\begin{array}{rrrrr}0 . & & 0 . & & \\ 0 & 3 & 14 & 0 & 3\end{array}$

0.0 .

$\begin{array}{llllll}0 & 4 & 0 & 4\end{array}$

1. $8287 E-03$

04120

0.418

0.419

0.511

0 .

0

$\begin{array}{ll}5 & 17\end{array}$

0611

0 .

$\begin{array}{lll}0 & 6 & 17\end{array}$

0 .

0712

0.718

$\begin{array}{rrr}0 . & & \\ 0 & 8 & 14\end{array}$

0 .

0 ? 11

$\begin{array}{rrr}0 . & 9 & 17\end{array}$

0.1015

0.
0 1114

0 .

$\begin{array}{lll}0 & 12 & 14\end{array}$

0.1315

$\begin{array}{rll}0 . & & \\ 0 & 14 & 17\end{array}$

0 .

$0 \quad 1618$

0 .
0.512

0.
0

0 .

$\begin{array}{lll}0 & 5 & 18 \\ 0 & 6 & 12\end{array}$

0.

0618

0.713

0.719

0.815

0 .

0912

0.918

0.1016

0.1115

0.12

0.1316

0.1418

0 .

01019

0.1013
0.117

0213

0.219

0.

0310

0.

0316

0.

0 48

0.

O 414

0.
0

$\begin{array}{cc}0 & 5 \\ 1.7021 E-03\end{array}$

0513

0.519

0 .

0.

0118

$\begin{array}{rrr}0 . & & \\ 0 & 2 & 8\end{array}$

0.
0 214

0.35

$2.6758 E-03$

$\begin{array}{ccc}0 & 311 \\ 0 . & & \end{array}$

0317

0.
0

0 .

0

0.

0.

0

0

068

$2.2167 E-03$

0614

0 .

0.
0

0.714

0.
0

0.816

0.913

0.919

0.1017

0.
0
0.16

0.1216

0.

$\begin{array}{lll}0 & 13 & 17\end{array}$

0.01419

0.

$0 \quad 1719$ 4. $7737 E-03$

0715

0.811

0

0817

0.

o 914

0.1012

0.1018

0.

$\begin{array}{lll}0 & 11 & 17\end{array}$

0 .

$\begin{array}{lll}0 & 1217\end{array}$

0.1318

0.1517

0 . $\begin{array}{rrrrr}0 . & & 0 . & & \\ 0 & 7 & 0 & 7 & 10\end{array}$
0.119

0.12

0.

0.

0.

o 312

0.

o 318

0.

0410

0

0416

$\begin{array}{rll}0 . & & \\ 0 & 5 & 9\end{array}$

0.515

0 .

069

0 .

0615

0.716
0

0.

0812

0 .

$0 \quad 818$

0.
0

0.

01013

0 .

01019

0 .

01118

0.

01218

$\begin{array}{rrr}0 . & & \\ 0 & 13 & 19\end{array}$

$\begin{array}{rrr}0 . & & \\ 0 & 15 & 18 \\ 0 . & & \end{array}$
0 .

024

$3.0875 E-03$

0210

0.216

$\begin{array}{rrr}0 . & & \\ 0 & 3 & 7\end{array}$

0 .

0313

0.319

0.
0

$\begin{array}{rll}0 . & & \\ 0 & 4 & 17\end{array}$

0.510

0.516

0 .

0610

0.616

$0 .-711$

0 .

$\begin{array}{lll}0 & 7 & 17\end{array}$

0.813

0 .

0819

0.

0916

0.

01014

0.

01113

0 .

01119

0 .

01219

0.

01416

0.

01519 


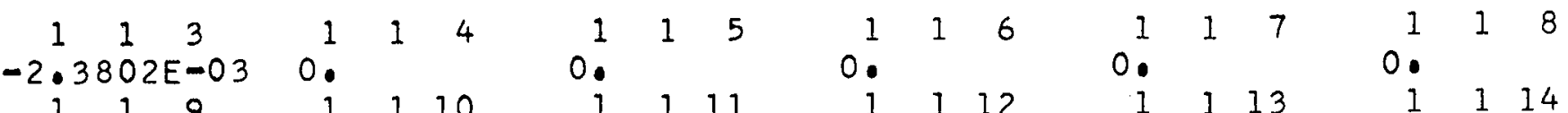

$\begin{array}{rrrr}1 & 1 & 9 & 1\end{array}$

$1115 \quad 0: 11$

0.1200

$\begin{array}{llll}1 & 2 & 5 & 1\end{array}$

$1211 \quad 1212$

0.0

$\begin{array}{llllll}1 & 2 & 17 & 1 & 2 & 18\end{array}$

$0 . \quad 0$

$\begin{array}{rrrr}1 & 3 & 8\end{array}$

$1314 \quad 1$

$\begin{array}{ccc}1 & 4 & 6\end{array}$

$\begin{array}{rrrrrr}1 & 4 & 12 & 1 & 4 & 13\end{array}$

$1418 \quad 1419$

$\begin{array}{rrrrr}0 . & & 0 . & & \\ 1 & 5 & 11 & 1 & 5\end{array}$

$\begin{array}{rrrr}0 . & & 0 . & \\ 1 & 517 & 1 & 518\end{array}$

0.50

$1611 \quad 1612$

$\begin{array}{rrrrrr}0 . & 0 & 0 & & \\ 1 & 6 & 17 & 1 & 6 & 18\end{array}$

$\begin{array}{rrrr}0.12 & 0.7713\end{array}$

$0: 1718$ 0 719

0

1814

0.911

0.

$\begin{array}{lll}1 & 9 & 17\end{array}$

$0.10 \quad 15$

0.

11114

0.

11214

0.1315

0 .

$\begin{array}{lll}1 & 14 & 17\end{array}$

0.

$116 \quad 18$

0 .
1111

0.

$\begin{array}{lll}1 & 1 & 17\end{array}$

1 27

0.

1

0

1

0

1

1

0.

0.

1414

0.

157

$-1.3944 E-03$

1513

0.

1519

0.

1613

0. 619

0.

1714

0.

1816

0.

1913

0.

1

0.1017

0.

11116

0.

11216

0.

$\begin{array}{lll}1 & 1317\end{array}$

0.

11419

0.1719

$116 \quad 19$
148

0.810
1112

0.118

0.

0

0

0 .

0 $-2.1921 E^{5}-03$

1311

0.

1317

0.49

0.

1

0.

415

0.

1

514

168

$-1.8160 E-03$

1614

0.

179

$-3.9108 E-03$

1

$\begin{array}{lll}1 & 8 & 11\end{array}$

0.

1817

0.

1914

0.1212

0.

11018

0.

$\begin{array}{lll}1 & 11 & 17\end{array}$

0.

$\begin{array}{lll}1 & 12 & 17\end{array}$

0.

11318

0.

11517
0.113

1119

0

129

0.1215

0.136

0.

$\begin{array}{lll}1 & 3 & 12\end{array}$

0.

1318

0.

1410

0.

0.

159

0.

1515

0.

169

0.

1

0

1

1

1

0.

0.

1

0.

11013

0.

11019

0.

11118

0.

$\begin{array}{lll}1 & 12 & 18\end{array}$

0.

11319

0.

11518
1416 $\begin{array}{lll}1 & 1 & 14\end{array}$

0.

124

$-2.5294 E-03$

1210

1216

$\begin{array}{rll}0 . & & \\ 1 & 3 & 7\end{array}$

0.313

1
0. 13

1319

$\begin{array}{rll}0 . & & \\ 1 & 4 & 11\end{array}$

0.417

1510

0.516

0.

1610

0.

1616

$\begin{array}{lll}1 & 7 & 11\end{array}$

0.

$\begin{array}{lll}1 & 7 & 17\end{array}$

0.

1813

$\begin{array}{rrr}0 & & \\ 1 & 8 & 19\end{array}$

0.

$\begin{array}{lll}1 & 9 & 16\end{array}$

0.

$110 \quad 14$

0 .

11113

0.

11119

0.

11219

0.

11416

0.

11519
0.417 
ATOM

CODE MASS FRACTION

ATOM

CODE

MASS FRACTION

LI $\quad 3.400000 E+00 \quad 1.000000 E+00$

\begin{tabular}{|c|c|c|c|c|}
\hline FORMULA & DENSITY & FUEL & DENSITY & TEMPERATURE \\
\hline $\mathrm{F}$ & RHO $\quad(G / C C)$ & RHOF & $(G / C C)$ & $T(D E G \quad K)$ \\
\hline $3.4100000 E+00$ & $5.2999986 E-01$ & 0. & & $2.9316000 \mathrm{E} \mathrm{O2}$ \\
\hline
\end{tabular}

$\begin{array}{cl}\text { COLLIDE } & \text { MULTIPLY } \\ \text { SGIN) } & \text { NUSGF }(N)\end{array}$

SGSO $(N, N)$ SGSIIN, SCATTER

$8.6686 E-02$

0.
0.
0.
0.

5.7936E-02

$7.8137 \mathrm{E}-02$

$1.0046 E-01$

$8.1623 E-02$

$6.3408 E-02$

0.

0 .

0 .

0.

$6.7501 E-02$

0 .

$9.5953 E-02$

0 .

$1.7377 E-01$

3. $0999 E-01$

$5.4519 E-01$

0.

0 .

0 .

$8.6598 E-01$

0 .

4. 1958E-02 1.9698E-02

5.0311E-02

4. $3215 E-02$

4. $1214 \mathrm{E}-02$

3. $1272 E-02$

2. $2816 E-02$

$2.1272 E-02$

$1.6719 E-02$

$1.6295 E-02$

SGSO $(N, N+1) \quad S G S 1(N, N+1)$

$2.4101 E-02$

1.3609E-02

$3.4515 E-02$

1. $8168 \mathrm{E}-02$

4. $7658 E-02$

$2.2761 E-02$

$4.3843 E-02-1.0782 E-02$

$5.0621 E-02-1.2903 E-02$

$4.7551 E-02-1.0963 E-02$

$3.6743 \mathrm{E}-02-1.0018 \mathrm{E}-02$

$3.6586 E-02-7.5610 E-03$

$6.8432 E-02$

$1.0918 \mathrm{E}-02$

$3.0640 E-02-4.9105 E-03$

4.0819E-02 -8.2321E-03

$5.1724 E-02-1.3099 E-02$

4. $8352 E-02$

8. $9125 E-03$

$1.0935 E-02-2.8666 E-03$

$8.9309 E-03-2.24 I I E-03$

4. $4239 E-02$

4. $4298 E-02$

$6.5930 E-03$

$6.6020 E-03-1.7620 E-03$

4. $4298 \mathrm{E}-02$

$6.3048 E-03$

$6.3048 E-03$

4. $2194 E-02$

$6.8727 E-03$

$6.3126 E-03-1.7038 E-03$

$3.7986 E-02$

$8.0085 E-03$

$6.3126 E-03-1.7038 E-03$

$8.4169 E-03-2.2717 E-03$

$1.2625 E-02-3.4075 E-03$

$3.7986 E-02$

$8.0085 E-03$

1. $2625 E-02-3.4075 E-03$

$3.7986 E-02$

$8.0085 E-03$

$1.2625 E-02-3.4075 E-03$

$2.2998 \mathrm{E} 00$

0.

$3.8352 \mathrm{E}-02$

8. $0112 E-03$

1.2259E-02 -3.2867E-03

$2.9478 E$ OO

5. $0611 E-02$

4. $9042 E-03$ 
$\operatorname{SGSL}(N 1, N 2)$

$\begin{gathered}0 \\ 6.98 \\ 0\end{gathered} \stackrel{3}{0}=04 \quad 0$

$\begin{array}{llllll}0 & 1 & 9 & 1 & 10\end{array}$

0 .

0

0 .

02

0.211

0.17

0.038

0.

0.06

3.0170E -05

0412

0

0418

0.511

0 .

$\begin{array}{lll}0 & 5 & 17\end{array}$

0.611

0.617

0.
0

0.718

$\begin{array}{lll}0 . & & \\ 0 & 8 & 14\end{array}$

0 .

0.911

$\begin{array}{rrr}0 . & & \\ 0 & 9 & 17\end{array}$

0.1015

0 .

o 1114

0 .

0 1214

0.1315

0 .

$\begin{array}{lll}0 & 14 & 17\end{array}$

0.1618

0 .
0

0

0

0

0 .

0212

0.
0

$\begin{array}{rrr}0 . & & \\ 0 & 3 & 9\end{array}$

0.315

$\begin{array}{rrr}0 . & 3 & 15 \\ 0 & 4 & 7\end{array}$

0 .

0413

0.419

0.
0

0.518

0 .

0612

0 .

0618

0.

0713

0.719

$\begin{array}{rrr}0 . & & \\ 0 & 8 & 15\end{array}$

0 .

0912

0.918

0.1016

0.1115

$\begin{array}{rll}0 . & 1215\end{array}$

0.1316

0 .

- 1418

0.1619

0 .
0

0 .

0117

$\begin{array}{rll}0 . & & \\ 0 & 2 & 7\end{array}$

0.13

0.19

0.

0.

0316

0.

0

04414

0.57

$1.6163 E-03$

0513

0 .

0519

0.

0613

0 .

0619

0 .

0714

0.810

$\begin{array}{lll}0 . & 8 & 10 \\ 0 & 8 & 16\end{array}$

0 .

0913

0.
0

$\begin{array}{lll}0 . & & \\ 0 & 10 & 17\end{array}$

0 .

01116

0.1216

0 .

01317 .

0.1419

0.

01719

0. $\begin{array}{lllllllll}0 & 1 & 6 & 0 & 1 & 7 & 0 & 1 & 8\end{array}$

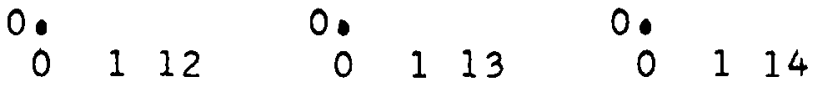

$\begin{array}{rrrrrrrr}0 . & & 0 & 0 & & 0 & & \\ 0 & 1 & 18 & 0 & 1 & 19 & 0 & 2\end{array}$

$0 . \quad 0 . \quad 5.1971 \mathrm{E}-04$

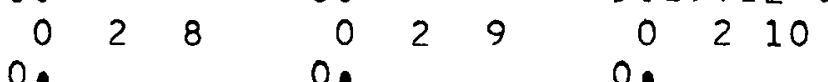

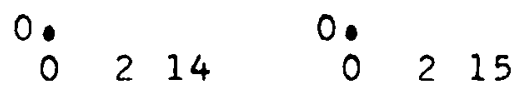

$\begin{array}{rrrrr}0 . & & 0 & 0 & \\ 0 & 3 & 5 & 0 & 3\end{array}$

$1.2548 E-03 \quad 0$.

$0311 \quad 0$

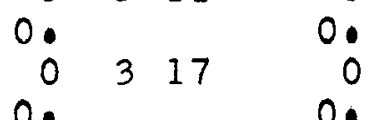

$3 \quad 12$

0 .

318

0410

0 .

0415

0.

0416

0.5

0.

0.59

0.

515

0 .

068

$2.5418 E-03$

0614

0 .

079

$1.9632 E-03$

0715

0.

0811

0.817

0 .

0914

0.1012

0.

0

69

0.615

0.

0710

0216

0.

037

0.313

0

0319

0411

0.

0417

0.510

$\begin{array}{rrr}0 . & & \\ 0 & 5 & 16\end{array}$

0 .

0610

0. 616

$\begin{array}{rrr}0 . & \\ 0 & 711\end{array}$

0.716

0.

o 812

0717

$\begin{array}{rrr}0 . & & \\ 0 & 8 & 18\end{array}$

0 .

0819

0 .

0.

0915

0916

0.1013

0.1014

0.1018

0.

01019

0.1113

0 .

$\begin{array}{lll}0 & 11 & 17\end{array}$

0.

01118

$\begin{array}{rrr}0 . & & \\ 0 & 1217\end{array}$

0.

o 1218

0.1318

0.

01319

0.

01517

01518

0 .

01119

0.

01219

0 .

01416

0

0 .

o 1519

0. 
$S G S L(N 1, N 2)$

$\begin{array}{llllll}1 & 1 & 3 & 1 & 1 & 4\end{array}$

$-6.3382 E-040$.

119

0.

1

0.

1

0.

1

0

0 .

1

0

1

$\begin{array}{llllll}1 & 4 & 0 & 0 & & \\ 1 & 4 & 7\end{array}$

$-2.9605 E-05$

1412

0.

1

0.

1

1

0

0 .

1617

0.

1

0.

1

0.

0.

1911

0 .

1917

0.

11015

0.

11114

0.

1. $12 \quad 14$

0.1315

$\begin{array}{rll}0.14 & 17\end{array}$

$n$

$116 \quad 18$

0 .
1110

1116

0.
1

0.

$\begin{array}{lll}1 & 2 & 12\end{array}$

0.

1218

0

0

1

0

0

0

1512

0 .

1518

0.

$\begin{array}{lll}1 & 6 & 12\end{array}$

$\begin{array}{rrr}0 . & & \\ 1 & 6 & 18\end{array}$

0.

1713

0.

1719

0

815

0

1912

$\begin{array}{lll}0 . & 9 & 18\end{array}$

n.

11016

11115

0 .

$1 \quad 1215$

0.

I. $13 \quad 16$

0.

11418

$$
0
$$

$116 \quad 19$
1419
115

1111

0.117

0.17

$\begin{array}{ccc}1 & 2 & 7 \\ 0 & & \end{array}$

1213

0.219

0.1219

0.16

1316

0.48

0.

1414

0.

157

$-1.3720 E-03$

1513

1519

1
1

$\begin{array}{rrr}0 . & & \\ 1 & 6 & 19\end{array}$

0.74

1714

0.810

0.

1816

0.

1913

0.

1919

$n$

$\begin{array}{lll}1 & 10 & 17\end{array}$

0.

11116

0.

I 1216

$\begin{array}{rll}0.13 & 17\end{array}$

0.

11419

0.1719 $\begin{array}{lllllllll}1 & 1 & 6 & 1 & 1 & 7 & 1 & 1 & 8\end{array}$

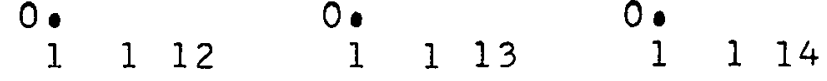

$\begin{array}{rrrrrr}0 & 1 & 0 & 18 & 1 & 1\end{array}$

0.

$\begin{array}{llllll}1 & 2 & 8 & 1 & 2 & 9\end{array}$

1 214

0.130 .136

$-1.1068 E-03$

$\begin{array}{ccc}1 & 311 \\ 0 & & \end{array}$

1317

0.149

0.15

0.

158

i 514

0.14

$\begin{array}{ccc}1 & 6 & 8 \\ -2.0269 E-03\end{array}$

1614

0.179

$-1.6542 E-03$

1715

0.811

$\begin{array}{lll}0 . & & \\ 1 & 8 & 17\end{array}$

0.

1914

0.

$1 \quad 10 \quad 12$

0.1018

0.1018

11117

$\begin{array}{lll}1 & 12 & 17\end{array}$

0.1318

0.

11517
1215

124

$-4.8123 E-04$

1210

1216

0.37

1. 37

1312

0.

$\begin{array}{lll}0 & 3 & 18\end{array}$

0.

1410

0. 416

0.

15

1515

0.

169

0.615

0 .

1710

0.716

0.812

0.818

1818

1915

0.

11013

0.1019

0.1819

$\begin{array}{lll}1 & 11 & 18\end{array}$

$\begin{array}{lll}1 & 12 & 18\end{array}$

$\begin{array}{rrr}0 . & & \\ 1 & 13 & 19\end{array}$

$\begin{array}{rll}01518 & \end{array}$

0.
1313

0.

1319

$\begin{array}{lll}0 & & \\ 1 & 11\end{array}$

0.417

1510

i 516

0.

1610

0.

1616

0. 711

$\begin{array}{rll}0 . & & \\ 1 & 7\end{array}$

0.

1819

0.

$\begin{array}{rll}0 . & & \\ 1 & 10 & 14\end{array}$

0 .

11113

0 .

11119

0.

11219

0.

11416

0.
0.813

1916

11519 


\begin{tabular}{clll}
$\begin{array}{c}\text { FORMULA } \\
\text { F }\end{array}$ & \multicolumn{1}{c}{ MASS } & FUEL MASS & TEMPERATURE \\
& A $($ OXY16=16) & AF $($ OXY16=16) & T (DEG K) \\
$4.0000000 E 02$ & $9.0150429 E 00$ & 0. & $2.9316000 E 02100$
\end{tabular}

CROSS SECTION (BARN)

COLLIDE
SGIN)

MULTIPLY

NUSGF $(N)$

0.

0.

0.

0.

0.

0.

0

0.

0.

0.

0.

0.

0.

0.

0.

0.
SCATTER

SGSO $(N, N) \quad S G S I(N, N) \quad S G S O(N, N+1) \quad S G S I(N, N+1)$

$1.0071 E$ 00 2.7584E-01 1.7340E-01 - I.6856E-01

9.3910E-01 3.4576E-01 4.2110E-01 -2.1129E-01

$1.5278 E$ OO $3.8886 E-01 \quad 6.8210 E-01-2.3763 E-01$

$1.3073 E$ OO $3.8886 E-01 \quad 5.4600 E-01-2.3763 E-01$

$1.9916 E 00 \quad 6.1873 E-01$ 1.1749E $00-3.7810 E-01$

$2.3419 E$ OO $7.6623 E-01 \quad 1.6081 E$ O0 $-4.6823 E-01$

$2.2326 E$ OO $7.3941 E-01$ 1.5904E $00-4.5184 E-01$

$2.6356 E$ 00 $8.4956 E=01 \quad 1.8774 E$ 00 $-5.1915 E-01$

4.8026E $00 \quad 5.5618 E-01$ 5.5740E-01 - $1.5686 E-01$

$5.3760 E$ O0 6.2259E-01 6.2400E-01 - $1.7559 E-01$

5.3760E OO 6.2259E-01 6.2400E-01 - $1.7559 E-01$

5.3760E $00 \quad 6.2259 E-01$ 6.2400E-01 - I.7559E-01

$5.3760 E$ OO 6.2259E-01 6.2400E-01 - $1.7559 E-01$

$5.1680 E$ OO $6.8112 E-01$ 8.3200E-01 -2.3412E-01

4.7520E $00 \quad 7.9817 E-01 \quad 1.2480 E$ OO $-3.5117 E-01$

4.6847E OO 7.8687E-01 1.2303E $00-3.4620 E-01$

4.2966E $00 \quad 7.2168 \mathrm{E}-01$ 1.1284E $00-3.1752 \mathrm{E}-01$

4.3508E $00 \quad 7.0435 E-01 \quad 1.0684 E \quad 00-3.0063 E-01$ 
$\begin{array}{rrrrrr}0 & 1 & 3 & 0 & 1 & 4 \\ 0.0 & 1 & 9 & 0 & 1 & 10\end{array}$

0.115

0.

025

$5.5620 E-01$

0211

0.

o 217

0.

038

$3.2300 E-02$

0314

0.46

0.412

$\begin{array}{rrr}0 . & & \\ 0 & 4 & 18\end{array}$

0.511

$\begin{array}{rrr}0 . & & \\ 0 & 5 & 17\end{array}$

0.611

0.617

$\begin{array}{rrr}0 & 6 & 17 \\ 0 & 7 & 12\end{array}$

$\begin{array}{rrr}0 . & & 12 \\ 0 & 7 & 18\end{array}$

$\begin{array}{rrr}0 . & & \\ 0 & 8 & 14\end{array}$

0.911

$\begin{array}{rrr}0 . & & \\ 0 & 9 & 17\end{array}$

0.1015

0.
0 1114

0.1214

0.
0 1315

0.1417

0.1618

0 .
0.16

0.

026

$5.5620 E-01$

० 212

$\begin{array}{rll}0 . & & \\ 0 & 2 & 18\end{array}$

0.39

0

0315

0.
0 47

0.
0 413

0.419

$\begin{array}{rrr}0 . & & \\ 0 & 5 & 12\end{array}$

0.

$\begin{array}{rrr}0 . & & \\ 0 & 6 & 12\end{array}$

0.618

$\begin{array}{rrr}0 & 6 & 18 \\ 0 . & & \\ 0 & 7 & 13\end{array}$

$\begin{array}{rrr}0 & 7 & 13 \\ 0 & & \\ 0 & 7 & 19\end{array}$

$\begin{array}{rrr}0 . & & \\ 0 & 8 & 15\end{array}$

0.
$0 \quad 912$

0.918

$\begin{array}{rrr}0 . & & \\ 0 & 10 & 16\end{array}$

0.1115

0.1215

0.1316

0.1316
0
0

0.1619

0 .
0
$5.5270 E-01$

$\begin{array}{cc}0 & 1 \\ 5.5270 E-01\end{array}$

0
0.
0
0.
0

1

0111

0112

0.

0117

0 .

0

0213

0.

0219

0.310

0

0316

0 .

048

0.414

$\begin{array}{rrr}0 . & & \\ 0 & 5 & 7\end{array}$

$\begin{array}{rrr}0 & 5 & 7 \\ 0 & & \\ 0 & 5 & 13\end{array}$

0.

0519

0.613

0.619

0 .

0714

0 .

0810

0.

o 816

0.913

0 .

o 919

0.51017

0 .

01116

0 .

01216

$\begin{array}{rll}0 . & 1317\end{array}$

0.

01419

0.

o 1719
0 .

028

0 .

0214

0 .

035

3.0600E-02

0311

0 .

0317

0.

049

0.415

$\begin{array}{rll}0 . & & \\ 0 & 5 & 8\end{array}$

$\begin{array}{rrr}0 . & & \\ 0 & 514\end{array}$

0 .

068

0.614

$\begin{array}{rrr}0 . & & \\ 0 & 7 & 9\end{array}$

0

0715

$\begin{array}{lll}0 . & & \\ 0 & 8 & 11\end{array}$

0.
0

$\begin{array}{rrr}0 . & \\ 0 & 9 & 14\end{array}$

0 .

01012

0 .

0 .

$\begin{array}{lll}0 & 11 & 17\end{array}$

0.

$\begin{array}{lll}0 & 12 & 17\end{array}$

0.1318

0 .

01517

0 .
01018
0113

0119

0.29

0.25

0

0.

036

3. $5570 E-01$

o 312

0.

0318

0.

0410

0.

0416

0.5

0.515

0.

069

0.615

0.

0710

0.

0716

0.812

0 .

0818

0.
0

0.1013

01013

01019

0.1118

0.1218

0.1319

0

01518

0 .
0.18

o 114

0.24

0.

0210

0216

037

$6 \cdot 1600 E-0$ ?

0313

0.

0319

0.411

0 .

0417

0510

0.516

0.

0610

0.616

0.711

$\begin{array}{rrr}0 . & & \\ 0 & 7 & 17\end{array}$

0 .

0813

0.819

0.
0

01014

0.01113

0 .

01119

0 .

$0 \quad 1219$

0.1416

0.1519

0. 


\begin{tabular}{|c|c|c|c|c|c|c|}
\hline ATOM & \multicolumn{2}{|c|}{ CODE } & MASS FR & AIOM & \multicolumn{2}{|c|}{ MASS FRACIION } \\
\hline$B E$ & \multicolumn{2}{|c|}{$4.000000 E+02$} & $1.000000 E+00$ & & & \\
\hline \multicolumn{3}{|c|}{$\begin{array}{l}\text { FORMULA } \\
\mathrm{F}\end{array}$} & DENSITY & $\begin{array}{cl}\text { FUEL } & \text { DENSI } \\
\text { RHOF } & 1 G / C C\end{array}$ & $\begin{array}{l}\text { TEMPE } \\
T 1 D\end{array}$ & TEMPERATURE \\
\hline \multicolumn{3}{|c|}{$4.0000000 E+02$} & $1.8499995 E \quad 00$ & 0. & \multicolumn{2}{|c|}{$2.9316000 E \quad 02101$} \\
\hline \multicolumn{7}{|c|}{ PROBABILITY/PATH (/CM) } \\
\hline $\begin{array}{l}\text { COL } \\
\text { SG }\end{array}$ & $\begin{array}{l}I D E \\
(N)\end{array}$ & $\begin{array}{l}\text { MULTIPLY } \\
\text { NUSGF (N) }\end{array}$ & $\operatorname{SGSO}(N, N)$ & \multicolumn{2}{|c|}{ SCATTER } & SGSI $(N, N+1)$ \\
\hline $\begin{array}{l}2.17 \\
2.44 \\
3.15 \\
2.33 \\
3.92\end{array}$ & $\begin{array}{l}76 E-01 \\
87 E-01 \\
27 E-01 \\
67 E-01 \\
05 E-01\end{array}$ & $\begin{array}{l}0 . \\
0 . \\
0 . \\
0 .\end{array}$ & $\begin{array}{l}1.2451 E-01 \\
1.1611 E-01 \\
1.8889 E-01 \\
1.6163 E-01 \\
2.4623 E-01\end{array}$ & $\begin{array}{l}3.4104 E-02 \\
4.2748 E-02 \\
4.8077 E-02 \\
4.8077 E-02 \\
7.6497 E-02\end{array}$ & $\begin{array}{l}2.1438 E-02 \\
5.2063 E-02 \\
8.4331 E-02 \\
6.7505 E-02 \\
1.4526 E-01\end{array}$ & $\begin{array}{l}-2.0840 E-02 \\
-2.6123 E-02 \\
-2.9379 E-02 \\
-2.9379 E-02 \\
-4.6746 E-02\end{array}$ \\
\hline $\begin{array}{l}4.88 \\
4.72 \\
5.57 \\
7.00 \\
7.41\end{array}$ & $\begin{array}{l}36 E-01 \\
56 E-01 \\
96 E-01 \\
04 E-01 \\
81 E-01\end{array}$ & $\begin{array}{l}0 . \\
0 . \\
0 . \\
0 .\end{array}$ & $\begin{array}{l}2.8954 E-01 \\
2.7603 E-01 \\
3.2585 E-01 \\
5.9377 E-01 \\
6.6466 E-01\end{array}$ & $\begin{array}{l}9.4733 E-02 \\
9.1417 E-02 \\
1.0504 E-01 \\
6.8763 E-02 \\
7.6973 E-02\end{array}$ & $\begin{array}{l}1.9882 E-01 \\
1.9663 E-01 \\
2.3211 E-01 \\
6.8914 E-02 \\
7.7148 E-02\end{array}$ & $\begin{array}{l}-5.7890 E-02 \\
-5.5864 E-02 \\
-6.4186 E-02 \\
-1.9393 E-02 \\
-2.1709 E-02\end{array}$ \\
\hline $\begin{array}{l}7.41 \\
7.41 \\
7.41 \\
7.41 \\
7.42\end{array}$ & $\begin{array}{l}81 E-01 \\
83 E-01 \\
86 E-01 \\
91 E-01 \\
00 E-01\end{array}$ & $\begin{array}{l}0 . \\
0 . \\
0 . \\
0 . \\
0 .\end{array}$ & $\begin{array}{l}6.6466 E-01 \\
6.6466 E-01 \\
6.6466 E-01 \\
6.3894 E-01 \\
5.8751 E-01\end{array}$ & $\begin{array}{l}7.6973 E-02 \\
7.6973 E-02 \\
7.6973 E-02 \\
8.4210 E-02 \\
9.8682 E-02\end{array}$ & $\begin{array}{l}7.7148 E-02 \\
7.7148 E-02 \\
7.7148 E-02 \\
1.0286 E-01 \\
1.5430 E-01\end{array}$ & $\begin{array}{l}-2.1709 E-02 \\
-2.1709 E-02 \\
-2.1709 E-02 \\
-2.8945 E-02 \\
-4.3417 E-02\end{array}$ \\
\hline $\begin{array}{l}7.31 \\
6.71 \\
6.70 \\
7.28\end{array}$ & $\begin{array}{l}51 E-01 \\
23 E-01 \\
35 E-01 \\
13 E-01\end{array}$ & $\begin{array}{l}0 . \\
0 . \\
0 . \\
0 .\end{array}$ & $\begin{array}{l}5.7919 E-01 \\
5.3121 E-01 \\
5.3791 E-01 \\
7.2703 E-01\end{array}$ & $\begin{array}{l}9.7284 E-02 \\
8.9225 E-02 \\
8.7083 E-02 \\
5.4164 E-02\end{array}$ & $\begin{array}{l}1.5211 \mathrm{E}-01 \\
1.3951 \mathrm{E}-01 \\
1.3209 \mathrm{E}-01\end{array}$ & $\begin{array}{l}-4.2802 E-02 \\
-3.9257 E-02 \\
-3.7168 E-02\end{array}$ \\
\hline
\end{tabular}


$S G S L(N 1, N 2)$

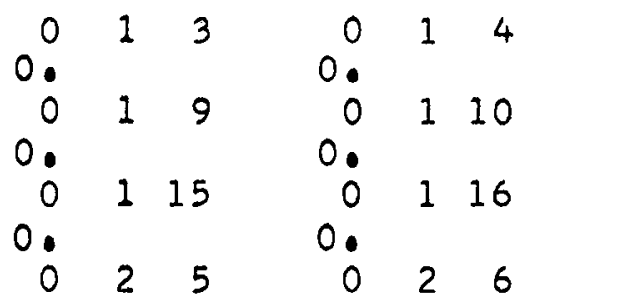

$6.8766 E-02$ 0211

0.

0

0

0
0

$3.9934 E-03$

0314

0 .

0.

0412

0.418

0.
0

0.

$\begin{array}{lll}0 & 517\end{array}$

0.
0 611

$\begin{array}{rrr}0 . & & \\ 0 & 6 & 17\end{array}$

$\begin{array}{rrr}0 . & & \\ 0 & 7 & 12\end{array}$

$\begin{array}{rrr}0 . & & \\ 0 & 718\end{array}$

$\begin{array}{rrr}0 . & & \\ 0 & 814\end{array}$

$\begin{array}{rrr}0 . & 9 & 11\end{array}$

$\begin{array}{rrr}0 . & & \\ 0 & 9 & 17\end{array}$

0.1015

0 .

$\begin{array}{lll}0 & 11 & 14\end{array}$

0.

$\begin{array}{lll}0 & 12 & 14\end{array}$

0 .

01315

0.1417
0.14

$\begin{array}{rrr}0 . & & \\ 0 & 16 & 18\end{array}$

0 .
$6.8766 \mathrm{E}-02$

0212

0.218

0 .

0

0

0 .

315

0.

0413

0.

0

0.

419

0

0

$5 \quad 12$

518

0612

0 .

o 618

$\begin{array}{lll}0 . & & \\ 0 & 7 & 13\end{array}$

0.

0

719

0.815

0 .

o 912

0 .

0

0.

01016

0 .

o 1115

0.

o 1215

0 .

01316

$\begin{array}{rll}0 . & & \\ 0 & 14 & 18\end{array}$

0 .

01619

0 .
047
0
6.
0
0.
0
0.
0
0.
0

$6.8333 E-02$

O 111

0.117

$\begin{array}{rrr}0 . & & \\ 0 & 2 & 7\end{array}$

0213

0.219

0.

0.310

0.316

$\begin{array}{rll}0 . & & \\ 0 & 4 & 8\end{array}$

0. 1414

$\begin{array}{rll}0 . & & \\ 0 & 5 & 7\end{array}$

0.513

$\begin{array}{rrr}0 . & & \\ 0 & 519\end{array}$

0

0613

0.619

$\begin{array}{rrr}0 . & & \\ 0 & 74\end{array}$

$\begin{array}{rrr}0 & 7 & 14 \\ 0 . & & \\ 0 & 8 & 10\end{array}$

0.816

0.913

0. 019

$\begin{array}{rrr}0 & 9 & 19 \\ 0 . & & \end{array}$

0.

01116

0. 1216

$\begin{array}{rrr}0 . & & \\ 0 & 1317\end{array}$

$\begin{array}{rrr}0 . & 14 & 19 \\ 0 & 1419\end{array}$

$\begin{array}{rrr}0 & 14 & 19 \\ 0 & 17 & 19\end{array}$

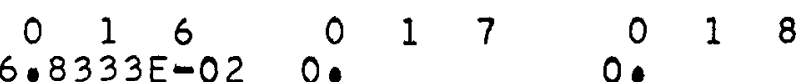

$\begin{array}{lllllllll}0 & 1 & 12 & 0 & 1 & 13 & 0 & 1 & 14\end{array}$

$0 . \quad 0$.

$\begin{array}{lllllllll}0 & 1 & 18 & 0 & 1 & 19 & 0 & 2 & 4\end{array}$

0.0 .000

$\begin{array}{llllllllll}0 & 2 & 8 & 0 & 2 & 9 & 0 & 2 & 10\end{array}$

$\begin{array}{rrrrrrrrr}0.14 & 0.0 & & 0 & 0 & & \\ 0 & 2 & 14 & 0 & 2 & 15 & 0 & 2 & 16\end{array}$

$\begin{array}{lllllllll}0 . & & 0 . & & 0 . & & \\ 0 & 3 & 5 & 0 & 3 & 6 & 0 & 3\end{array}$

$3.7832 E-03 \quad 4.3977 E-02 \quad 7.6159 E-03$

$\begin{array}{lllllllll}0 & 311 & 0 & 3 & 12 & 0 & 3 & 13\end{array}$

0.0 .00

0317

0.49

0.
0

0.
0

0.514

0.

$\begin{array}{lll}0 & 6\end{array}$

0.614

0.
0

$\begin{array}{rrr}0 . & & \\ 0 & 7 & 15\end{array}$

0.811

$\begin{array}{rrr}0 . & & \\ 0 & 8 & 17\end{array}$

0.9
0

0.1012

0.18

$\begin{array}{rrr}0 . & & \\ 0 & 11 & 17\end{array}$

$\begin{array}{rrr}0 . & & \\ 0 & 12 & 17\end{array}$

0.1318

$\begin{array}{rrr}0 . & & \\ 0 & 15 & 17\end{array}$

0 .
0318

0.

0410

0.416

$\begin{array}{rrr}0 . & & \\ 0 & 5 & 9\end{array}$

0.515

0.

069

0.

0615

0.710

0. 716

$\begin{array}{rrr}0 . & & \\ 0 & 812\end{array}$

0.818

$\begin{array}{rrr}0 . & & \\ 0 & 9 & 15\end{array}$

0.1013

0.1019

0.

$0 \quad 1118$

0 .

01218

0.

$0 \quad 1319$

0.1518

0.
0.319

0.411

$\begin{array}{rll}0 . & & \\ 0 & 4 & 17\end{array}$

0.510

0.516

0.

0610

0.616

0.711
0

0.717

0.

$0 \quad 813$

$\begin{array}{rrr}0 . & & \\ 0 & 8 & 19\end{array}$

0.916

0.1014

$0 . \quad 14$

$0 \quad 1113$

0.

$0 \quad 1119$

0.

01219

0.1416

0.

$0 \quad 1519$

0 


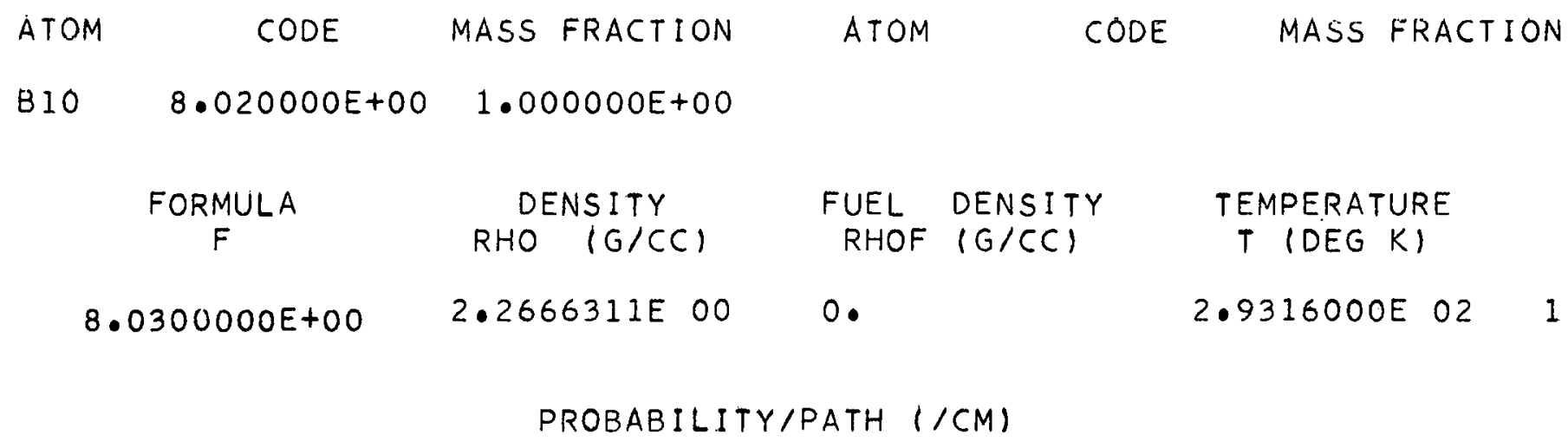

$\begin{array}{ll}\text { COLLIDE } & \text { MULTIPLY } \\ \text { SGIN) } & \text { NUSGF }(N)\end{array}$

\begin{tabular}{|c|c|}
\hline $\begin{array}{l}2.3589 E-01 \\
2.7204 E-01 \\
3.3110 E-01 \\
3.6296 E-01 \\
4.3977 E-01\end{array}$ & $\begin{array}{l}0 . \\
0 . \\
0 . \\
0 . \\
0 .\end{array}$ \\
\hline $\begin{array}{l}6.5129 \mathrm{E}-01 \\
7.9834 \mathrm{E}-01 \\
8.4507 \mathrm{E}-01 \\
1.0592 \mathrm{E} \quad 00 \\
1.7136 \mathrm{E} \quad 00\end{array}$ & $\begin{array}{l}0 . \\
0 . \\
0 . \\
0 . \\
0 .\end{array}$ \\
\hline $\begin{array}{lll}3.4359 E & 00 \\
8.1679 E & 00 \\
2.1157 E & O 1 \\
4.3955 E & 01 \\
8.3318 E & 01\end{array}$ & $\begin{array}{l}0 . \\
0 . \\
0 . \\
0 . \\
0 .\end{array}$ \\
\hline $\begin{array}{ll}1.3701 E & 02 \\
2.2552 \mathrm{E} & 02 \\
3.7697 \mathrm{E} & 02 \\
4.8542 \mathrm{E} & 02\end{array}$ & $\begin{array}{l}0 . \\
0 . \\
0 . \\
0 .\end{array}$ \\
\hline
\end{tabular}

\section{PROBABILITY/PATH I/CMI}

SGSO $(N, N) \quad$ SGSIIN,N) SGSTER $(N, N+1)$ SGSI(N,N+1)

$1.2733 E-01 \quad 3.5893 E-02 \quad 7.7175 E-02-2.2151 E-02$

$1.4431 E-01 \quad 4.0679 E-02 \quad 8.7465 E-02-2.5104 E-02$

$1.7402 E-01 \quad 4.9054 E-02 \quad 1.0547 E-01-3.0272 E-02$

$1.8463 E-01 \quad 5.2046 E-02 \quad 1.1190 E-01-3.2118 E-02$

$2.2071 E-01 \quad 6.2215 E-02 \quad 1.3377 E-01-3.8394 E-02$

$3.3743 E-01 \quad 9.5118 E-02 \quad 2.0451 E-01-5.8699 E-02$

4.0959E-01 1.1546E-01 2.4825E-01 -7.1251E-02

4.1383E-01 $1.1665 E-01 \quad 2.5082 E-01-7.1989 E-02$

$6.1738 E-01 \quad 6.4268 E-02 \quad 6.4312 E-02-1.8459 E-02$

$6.2355 E-01 \quad 6.4911 E-02 \quad 6.4955 E-02-1.8643 E-02$

5.8651E-01 6.1055E-02 6.1097E-02 - 1.7536E-02

$5.2477 E-01 \quad 5.4628 E-02 \quad 5.4665 E-02-1.5690 E-02$

4.9390E-01 5.1415E-02 5.1450E-02 -1.4767E-02

4.7675E-01 5.6337E-02 6.8600E-02 -1.9689E-02

$4.4245 E-01 \quad 6.6182 E-02 \quad 1.0290 E-01-2.9534 E-02$

4.4245E-01 6.6182E-02 1.0290E-01 -2.9534E-02

$4.4245 E-01 \quad 6.6182 E-02 \quad 1.0290 E-01-2.9534 E-02$

$4.4782 E-01 \quad 6.4641 E-02 \quad 9.7530 E-02-2.7993 E-02$ 


\begin{tabular}{|c|c|c|c|c|c|}
\hline ATOM & CODE & MASS FRACTION & ATOM & CODE & MASS FRACTION \\
\hline \multirow[t]{3}{*}{$B$} & $8.000000 E+00$ & $1.000000 E+00$ & & & \\
\hline & . & & - & & \\
\hline & $\begin{array}{c}\text { FORMULA } \\
\text { F }\end{array}$ & $\begin{array}{c}\text { DENSITY } \\
\text { RHO }(G / C C)\end{array}$ & $\begin{array}{l}\text { FUEL } \\
\text { RHOF }\end{array}$ & $\begin{array}{l}\text { DENSITY } \\
(G / C C)\end{array}$ & $\begin{array}{c}\text { TEMPERATURE } \\
T(D E G \quad K)\end{array}$ \\
\hline & $100000 E+00$ & $2.4500007 E \quad 00$ & 0. & & $2.9316000 E \quad 02$ \\
\hline
\end{tabular}

PROBABILITY/PATH (/CM)

\begin{tabular}{|c|c|c|c|c|c|}
\hline \multirow{2}{*}{$\begin{array}{c}\text { COLLIDE } \\
\text { SG(N) }\end{array}$} & MULTIPLY & \multicolumn{4}{|c|}{ SCATTER } \\
\hline & NUSGF $(N)$ & $\operatorname{SGSO}(N, N)$ & $\operatorname{SGSI}(N, N)$ & SGSO $(N, N+1)$ & $\operatorname{SGSI}(N, N+1)$ \\
\hline $\begin{array}{l}5.5159 E-01 \\
5.5326 E-01 \\
5.5540 E-01 \\
5.5819 E-01 \\
5.6174 E-01\end{array}$ & $\begin{array}{l}0 . \\
0 . \\
0 . \\
0 . \\
0 .\end{array}$ & $\begin{array}{l}3.5444 E-01 \\
3.5444 E-01 \\
3.5444 E-01 \\
3.5444 E-01 \\
3.5444 E-01\end{array}$ & $\begin{array}{l}8.9470 E-02 \\
8.9470 E-02 \\
8.9470 E-02 \\
8.9470 E-02 \\
8.9470 E-02\end{array}$ & $\begin{array}{l}1.9124 E-01 \\
1.9124 E-01 \\
1.9124 E-01 \\
1.9124 E-01 \\
1.9124 E-01\end{array}$ & $\begin{array}{l}-5.5584 E-02 \\
-5.5584 E-02 \\
-5.5584 E-02 \\
-5.5584 E-02 \\
-5.5584 E-02\end{array}$ \\
\hline $\begin{array}{l}5.6628 E-01 \\
5.7215 E-01 \\
5.7967 E-01 \\
6.1680 E-01 \\
7.3880 E-01\end{array}$ & $\begin{array}{l}0 . \\
0 . \\
0 . \\
0 . \\
0 .\end{array}$ & $\begin{array}{l}3.5444 E-01 \\
3.5444 E-01 \\
3.5444 E-01 \\
4.9787 E-01 \\
4.9787 E-01\end{array}$ & $\begin{array}{l}8.9470 E-02 \\
8.9470 E-02 \\
8.9470 E-02 \\
4.7782 E-02 \\
4.7782 E-02\end{array}$ & $\begin{array}{l}1.9124 E-01 \\
1.9124 E-01 \\
1.9124 E-01 \\
4.7810 E-02 \\
4.7810 E-02\end{array}$ & $\begin{array}{l}84 E-02 \\
84 E-02 \\
84 E-02 \\
96 E-02 \\
96 E-02\end{array}$ \\
\hline 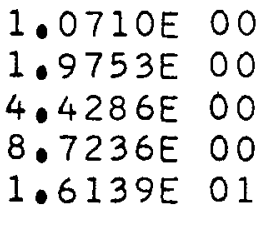 & $\begin{array}{l}0 . \\
0 . \\
0 . \\
0 . \\
0 .\end{array}$ & $\begin{array}{l}4.9787 E-01 \\
4.9787 E-01 \\
4.9787 E-01 \\
4.8193 E-01 \\
4.5006 E-01\end{array}$ & $\begin{array}{l}4.7782 E-02 \\
4.7782 E-02 \\
4.7782 E-02 \\
5.2414 E-02 \\
6.1678 E-02\end{array}$ & $\begin{array}{l}4.7810 E-02 \\
4.7810 E-02 \\
4.7810 E-02 \\
6.3747 E-02 \\
9.5620 E-02\end{array}$ & $\begin{array}{l}-1.3896 E-02 \\
-1.3896 E-02 \\
-1.3896 E-02 \\
-1.8528 E-02 \\
-2.7792 E-02\end{array}$ \\
\hline 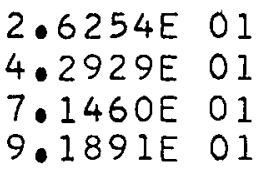 & $\begin{array}{l}0 . \\
0 . \\
0 . \\
0 .\end{array}$ & $\begin{array}{l}4.5006 E-01 \\
4.5006 E-01 \\
4.5505 E-01 \\
5.4568 E-01\end{array}$ & $\begin{array}{l}6.1678 E-02 \\
6.1678 E-02 \\
6.0228 E-02 \\
3.3887 E-02\end{array}$ & $\begin{array}{l}9.5620 E-02 \\
9.5620 E-02 \\
9.0630 E-02\end{array}$ & $\begin{array}{l}-2.7792 E-02 \\
-2.7792 E-02 \\
-2.6342 E-02\end{array}$ \\
\hline
\end{tabular}


ATOM

CODE

MASS FRACTION

ATOM

CODE

MAOS FRACTION

c

$3.300000 E+00 \quad 1.000000 E+00$

\begin{tabular}{|c|c|}
\hline \multicolumn{2}{|c|}{$\begin{array}{c}\text { FORMULA } \\
\mathrm{F}\end{array}$} \\
\hline \multicolumn{2}{|c|}{$3.2100000 \mathrm{H}+00$} \\
\hline $\begin{array}{l}\text { COLLIDE } \\
\text { SG(N) }\end{array}$ & $\begin{array}{l}\text { MULTIPLY } \\
\text { NUSGF (N) }\end{array}$ \\
\hline $\begin{array}{l}1.1434 E-01 \\
1.1835 E-01 \\
1.4844 E-01 \\
1.6248 E-01 \\
2.0260 E-01\end{array}$ & $\begin{array}{l}0 . \\
0 . \\
0 . \\
0 . \\
0 .\end{array}$ \\
\hline $\begin{array}{l}2.5154 E-01 \\
2.9086 E-01 \\
3.2215 E-01 \\
3.5705 E-01 \\
3.7711 E-01\end{array}$ & $\begin{array}{l}0 . \\
0 . \\
0 . \\
0 . \\
0 .\end{array}$ \\
\hline $\begin{array}{l}3.7711 E-01 \\
3.7711 E-01 \\
3.7712 E-01 \\
3.8114 E-01 \\
3.8517 E-01\end{array}$ & $\begin{array}{l}0 . \\
0 . \\
0 . \\
0 . \\
0 .\end{array}$ \\
\hline $\begin{array}{l}3.8520 E-01 \\
3.8524 E-01 \\
3.8858 E-01 \\
3.7549 E-01\end{array}$ & $\begin{array}{l}0 . \\
0 . \\
0 \\
0 .\end{array}$ \\
\hline
\end{tabular}

DENSITY
RHO (G/CC)

FUEL DENSITY

RHOF (G/CC)

TEMPERATURE

$T$ (DEG K)

$1.6000005 E 00 \quad 0$.

$2.9316000 E \quad 02 \quad 1$

PROBABILITY/PATH (/CM)

\begin{tabular}{|c|c|c|c|}
\hline \multicolumn{4}{|c|}{ SCATTER } \\
\hline$G S O(N, N)$ & $\operatorname{SGS} 1(N, N)$ & $N, N+1)$ & SGS1 $(N, N+1)$ \\
\hline $\begin{array}{l}02 \\
02 \\
01 \\
01\end{array}$ & $\begin{array}{l}5 E-02 \\
6 E-02 \\
2 E-02 \\
5 E-02 \\
4 E-02\end{array}$ & $\begin{array}{l}E-02 \\
E-02 \\
E-02 \\
E-02 \\
-02\end{array}$ & $\begin{array}{l}-1 \\
-1 \\
-1 \\
-1 \\
-1\end{array}$ \\
\hline $\begin{array}{l}7 E-01 \\
9 E-01 \\
3 E-01 \\
7 E-01\end{array}$ & $\begin{array}{l}4 E-02 \\
3 E-02 \\
O E-02 \\
5 E-02 \\
9 E-02\end{array}$ & $\begin{array}{l}E-02 \\
E-02 \\
E-01 \\
E-02 \\
E-02\end{array}$ & $\begin{array}{l}3 E-02 \\
E-02 \\
E-02 \\
E-03 \\
E-03\end{array}$ \\
\hline $\begin{array}{l}3.4714 E-01 \\
3.4714 E-01 \\
3.4714 E-01 \\
3.4073 E-01 \\
3.2391 E-01\end{array}$ & $\begin{array}{l}2.9959 E-02 \\
2.9959 E-02 \\
2.9959 E-02 \\
3.3256 E-02 \\
3.9625 E-02\end{array}$ & $\begin{array}{l}2.9973 E-02 \\
2.9973 E-02 \\
2.9973 E-02 \\
4.0389 E-02 \\
6.1222 E-02\end{array}$ & $\begin{array}{l}-8.8409 E-03 \\
-8.8409 E-03 \\
-8.8409 E-03 \\
-1.1913 E-02 \\
-1.8058 E-02\end{array}$ \\
\hline $\begin{array}{l}3.2391 E-01 \\
3.2391 E-01 \\
3.2988 E-01 \\
3.7526 E-01\end{array}$ & $\begin{array}{l}3.9625 E-02 \\
3.9625 E-02 \\
3.9011 E-02 \\
2.1015 E-02\end{array}$ & $\begin{array}{l}6.1222 E-02 \\
6.1222 E-02 \\
5.8519 E-02\end{array}$ & $\begin{array}{l}-1.8058 E-02 \\
-1.8058 E-02 \\
-1.7261 E-02\end{array}$ \\
\hline
\end{tabular}


ATOM

CODE

MASS FRACTION

ATOM

CODE

MASS FRACTION

NA

$$
7.300000 E+00 \quad 1.000000 E+00
$$

FORMULA

$F$

$7.3100000 E+00$

\author{
DENSITY \\ RHO (G/CC)
}

$\begin{array}{ll}\text { FUEL } & \text { DENSITY } \\ \text { RHOF } & (G / C C)\end{array}$

0.
TEMPERATURE
$T(D E G K)$

$2.9316000 E \quad 02 \quad 1$

$$
T(D E G K)
$$

PROBABILITY/PATH (/CM)

$\begin{array}{cl}\text { COLLIDE } & \text { MULTIPLY } \\ \text { SG(N) } & \text { NUSGF(N) }\end{array}$

$5.5343 E-02$

5. $9160 E-02$

$6.8702 E-0.2$

$5.8525 E-02$

$9.2875 E-02$

$1.2977 E-01$

$9.5421 E-02$

$1.0051 E-01$

$1.0688 \mathrm{E}-01$

$6.4250 E-01$

$6.3619 E-01$

$7.7785 E-02$

$7.9999 E-02$

$8.1807 E-02$

$8.4514 E-02$

8.7557E-02

$9.0146 E-02$

$9.3475 E-02$

$9.7273 E-02$
0.

0.

0.

0 .

0.

0.

0.

0 .

0 .

0 .

0.

0 .

0 .

0.

0 .

0.

0 .

0.

0.

SCATTER

SGSO $(N, N) \quad S G S I(N, N) \quad S G S O(N, N+1) \quad S G S I(N, N+1)$

4. 5924E-02

4. $9091 E-02$

4.5699E-03 9.4189E-03 -2.9539E-03

$5.7009 E-02$

$4.8563 E-02$

4. $8850 E-03$

$1.0068 E-02-3.1576 E-03$

$5.6728 E-03 \quad 1.1692 E-02-3.6667 E-03$

$7.7067 E-02$

$4.8325 E-03$

$9.9602 E-03-3.1236 E-03$

$7.6690 E-03 \quad 1.5806 E-02-4.9571 E-03$

$1.0768 E-01$

$1.0715 E-02$

$2.2086 E-02-6.9262 E-03$

7. $9178 E-0$ ?

$7.8792 E-03$

$1.6239 E-02-5.0930 E-03$

8. $3401 E-02$

$8.2995 E-03$

$1.7106 E-02-5.3646 E-03$

$1.0232 E-01$

4. 5466E-03

4.5470E-03-1.4260E-03

6. $1514 E-01$

2. $7333 E-02$

$2.7336 E-02-8.5726 E-03$

$6.0905 E-01$

$2.7062 E-02$

$2.7066 E-02-8.4878 E-03$

$7.4305 E-02$

$3.3016 E-03$

$3.3020 E-03-1.0355 E-03$

$7.6132 E-02$

$3.3828 E-03$

$3.3832 E-03-1.0609 E-03$

$7.6204 \mathrm{E}-02$

$3.7962 E-03$

4.5831E-03-1.4373E-03

$7.5542 E-02$

4.6144E-03

$7.0263 E-03-2.2035 E-03$

$7.7172 E-02$

4.7139E-03

$7.1778 E-03-2.2509 E-03$

$7.7637 E-02$

$4.7425 E-03$

$7.7802 \mathrm{E}-02$

$4.6116 E-03$

$7.2211 E-03-2.2646 E-03$

$8.5876 \mathrm{E}-02$

$2.5076 E-03$ 


\begin{tabular}{|c|c|c|c|c|c|}
\hline ATOM & CODE & MASS FRACTION & ATOM & CODE & MASS FRACTION \\
\hline MG & $5.140000 E+00$ & $1.000000 E+00$ & & & \\
\hline & $\begin{array}{l}\text { FORMULA } \\
F\end{array}$ & $\begin{array}{c}\text { DENSITY } \\
\text { RHO }(G / C C)\end{array}$ & $\begin{array}{l}\text { FUEL } \\
\text { RHOF }\end{array}$ & $\begin{array}{l}\text { DENSITY } \\
(G / C C)\end{array}$ & $\begin{array}{c}\text { TEMPERATURE } \\
T(D E G K\rangle\end{array}$ \\
\hline & $500000 E+00$ & $1.7399992 \mathrm{E} \quad 00$ & 0. & & $2.9316000 E \quad 02$ \\
\hline
\end{tabular}

PROBABILITY/PATH (/CM)

$\begin{array}{cc}\text { COLLIDE } & \text { MULT } \\ \text { SGIN) } & \text { NUS } \\ 7.0045 E-02 & 0 . \\ 7.5433 E-02 & 0 . \\ 9.8063 E-02 & 0 . \\ 1.1530 E-01 & 0 . \\ 1.2824 E-01 & 0 . \\ 1.8535 E-01 & 0 . \\ 2.9419 E-01 & 0 . \\ 3.7609 E-01 & 0 . \\ 2.6940 E-01 & 0 . \\ 1.4656 E-01 & 0 . \\ 1.4657 E-01 & 0 . \\ 1.4659 E-01 & 0 . \\ 1.4666 E-01 & 0 . \\ 1.4677 E-01 & 0 . \\ 1.4697 E-01 & 0 . \\ & \\ 1.4723 E-01 & 0 . \\ 1.4767 E-01 & 0 . \\ 1.4316 E-01 & 0 . \\ 1.3772 E-01 & 0 .\end{array}$

SGSO $(N, N) \quad$ SGSI $(N, N)$ SGSO $(N, N+1) \quad S G S I(N, N+I)$

$5.8759 E-02$

$6.3279 E-02$

$8.2263 E-02$

$9.6726 \mathrm{E}-02$

$1.0757 \mathrm{E}-01$

$1.5549 E-01$

$2.4679 E-01$

3.1549E-0 1

$2.5855 E-01$

$1.4065 E-0 I$

$1.4065 E-01$

$1.4065 E-01$

$1.4065 E-01$

$1.3868 \mathrm{E}-01$

$1.3475 E-01$

$1.3475 E-01$

$1.3475 E-01$

$1.3050 E-01$

$1.3531 E-01$
$5.4850 E-03$

$5.9068 E-03$

$7.6789 E-03$

$9.0288 E-03$

$1.0041 E-02$

$1.4513 E-02$

$2.3037 E-02$

$2.9451 E-02$

$1.0850 E-02$

$5.9026 E-03$

$5.9026 E-03$

$5.9026 E-03$

$5.9026 E-03$

$6.5219 E-03$

$7.7604 E-03$

$7.7604 E-03$

$7.7604 \mathrm{E}-03$

$7.2945 E-03$

$3.7347 E-03$
1. $1286 \mathrm{E}-02-3.5517 \mathrm{E}-03$

1.2154E-02 -3.8249E-03

$1.5800 E-02-4.9723 E-03$

$1.8578 E-02-5.8464 E-03$

$2.0662 E-02-6.5021 E-03$

$2.9864 E-02-9.3979 E-03$

4.7400E-02 - $1.4917 E-02$

$6.0596 E-02-1.9071 E-02$

$1.0852 E-02-3.4149 E-03$

$5.9033 E-03-1.8577 E-03$

$5.9033 E-03-1.8577 E-03$

$5.9033 E-03-1.8577 E-03$

$5.9033 E-03-1.8577 E-03$

$7.8710 E-03-2.4770 E-03$

$1.1807 E-02-3.7154 E-03$

1. $1807 E-02-3.7154 E-03$

$1.1807 E-02-3.7154 E-03$

$1.0788 E-02-3.3949 E-03$ 


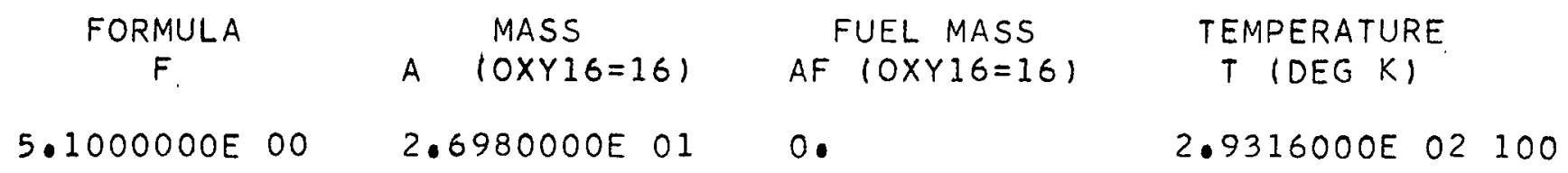

CROSS SECTION (BARN)

\begin{tabular}{|c|c|}
\hline \multicolumn{2}{|c|}{$\begin{array}{l}\text { COLLIDE } \\
\text { SG }(N)\end{array}$} \\
\hline $\begin{array}{l}1.9500 E \\
2.1750 E \\
2.8250 E \\
2.7500 E \\
2.6500 E\end{array}$ & $\begin{array}{ll}0 & 0 \\
0 & 0 \\
0 & 0 \\
0 & 0 \\
0 & 0\end{array}$ \\
\hline $\begin{array}{l}4: 3000 E \\
3.7251 \mathrm{E} \\
4.9751 \mathrm{E} \\
4.7702 \mathrm{E} \\
1.4454 \mathrm{E}\end{array}$ & $\begin{array}{ll}0 & 0 \\
0 & 0 \\
0 & 0 \\
0 & 0 \\
0 & 0\end{array}$ \\
\hline $\begin{array}{l}1.3512 \mathrm{E} \\
1.3532 \mathrm{E} \\
1.3537 \mathrm{E} \\
1.3983 \mathrm{E} \\
1.4748 \mathrm{E}\end{array}$ & $\begin{array}{ll}0 & 0 \\
0 & 0 \\
0 & 0 \\
0 & 0 \\
0 & 0\end{array}$ \\
\hline $\begin{array}{l}1.5024 \mathrm{E} \\
1.5046 \mathrm{E} \\
1.5484 \mathrm{E} \\
1.6793 \mathrm{E}\end{array}$ & $\begin{array}{ll}0 & 0 \\
00 \\
00 \\
0 & 0\end{array}$ \\
\hline
\end{tabular}

MULT I PLY

NUSGF (N)

0.

0.

0.

0.

0 .

0.

0.

0.

0.

0.

0.

0.

0.

0.

0.

0.

0.

0.

0.

\begin{tabular}{|c|c|}
\hline$G S O(N, N)$ & $\operatorname{SGSI}(N, N)$ \\
\hline $\begin{array}{l}5.4375 E-01 \\
5.3450 E-01 \\
6.2070 E-01 \\
6.2498 E-01 \\
7.7965 E-01\end{array}$ & $\begin{array}{l}4.255 I E-0 I \\
4.4870 E-0 I \\
5.5573 E-01 \\
5.4778 E-01 \\
5.9075 E-01\end{array}$ \\
\hline $\begin{array}{lll}1.0389 E & 00 \\
9.4107 E-01 \\
4.2458 E & 00 \\
4.5962 E & 00 \\
1.3923 E & 00\end{array}$ & $\begin{array}{l}8.8018 E-01 \\
7.7829 E-01 \\
3.5534 E-01 \\
1.7379 E-01 \\
5.2647 E-02\end{array}$ \\
\hline $\begin{array}{ll}1.3008 E & 00 \\
1.3008 E & 00\end{array}$ & $\begin{array}{l}6 E-02 \\
6 E-02\end{array}$ \\
\hline $\begin{array}{ll}1.2960 E & 00 \\
1.3130 E & 00 \\
1.3351 E & 00\end{array}$ & $\begin{array}{l}4.9003 E-02 \\
5.5584 E-02 \\
6.9072 E-02\end{array}$ \\
\hline $\begin{array}{ll}1.3397 E & 00 \\
1.3072 E & 0 O \\
1.2940 E & 00 \\
1.4753 E & 00\end{array}$ & $\begin{array}{l}6.9313 E-02 \\
6.7630 E-02 \\
6.5001 E-02 \\
3.6735 E-02\end{array}$ \\
\hline
\end{tabular}

SCATTER SGSO $(N, N+1) \quad S G S 1(N, N+1)$

$7.9022 E-01 \quad 2.8454 E-01$

7.7678E-01 4.1347E-01

$9.0204 E-01 \quad 6.3255 E-01$

$9.0827 E-01 \quad 5.8513 E-01$

$1.1330 E$ OO 3.2284E-01

$1.5098 E$ 00 8.3896E-01

$1.3676 \mathrm{E}$ 00 $6.7649 \mathrm{E}-01$

$7.2922 E-01-2.3074 E-01$

$1.7380 E-01-5.5015 E-02$

$5.2651 E-02-1.6666 E-02$

4. $9190 E-02-1.5571 E-02$

4.9190E-02 - $1.5571 E-02$

4. $9.008 E-02-1.5513 E-02$

$6.7044 E-02-2.1222 E-02$

$1.0494 E-01-3.3216 E-02$

$1.0530 E-01-3.3333 E-02$

$1.0275 E-01-3.2521 E-02$

$9.6008 E-02-3.0390 E-02$

RELIABILITY LIMITATION: First-significant-figure uncertainty in high energy scatter cross sections of aluminum. 
$\operatorname{SGSL}(N 1, N 2)$

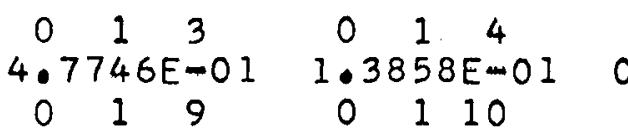

0.

0

115

0

0

116

026

025

$2.8576 E-01$

0211

0

217

0.

$\begin{array}{llll}0 & 3 & 0\end{array}$

$1 \cdot 2208 E-01$

0314

0.

046

5. 5089E-01

0412

0

418

0.

0

0.

0

0

0

0.

0

0

0

0

0911

0 .

0

0.

0

0

0.

01214

0.

01315

0 .

$0 \quad 14 \quad 17$

0 .

01618

0 .
$1.0672 E-01$

0212

0

218

0.

039

$9.9956 E-02$

0.

$3.3413 E-01$

0413

0.

419

0 .

$\begin{array}{lll}0 & 5 & 12\end{array}$

0 .

0

518

0.

0612

0.018

0.713

0.

719

0

0815

0 .

0912

$\begin{array}{rrr}0 . & & \\ 0 & 9 & 18\end{array}$

0

01115

0 .

01215

0.1316

0.

01418

0.

01619

0 .
047

0.1016

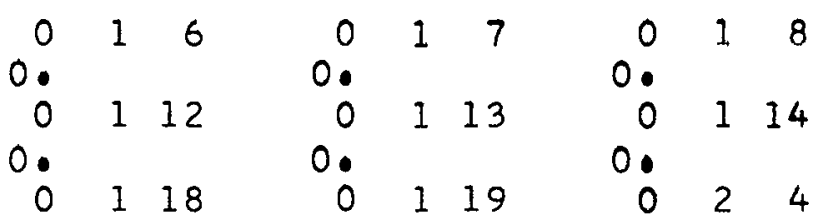

0.
0

0.

029

0.

021

$\begin{array}{rrrrr}0 . & & & 0 . & \\ 0 & 3 & 6 & 0 & 3\end{array}$

0

0

214

0.35

$5.4712 E-01$

0311

0310

0.

0316

0317

0 .

048

$2.0266 E-01$

049

1.2907E-01

$3.3184 E-01$

0312

0 .

0318

0.

0410

0.57

$6.4492 E-01$

0513

0.

0519

0.

0613

0.
0

$\begin{array}{rrr}0 . & & \\ 0 & 7 & 14\end{array}$

$\begin{array}{rrr}0 . & & \\ 0 & 8 & 10\end{array}$

$\begin{array}{rrr}0 . & & \\ 0 & 8 & 16\end{array}$

0.913

0.13

0919

0.1017

0.16

01116

01216

$\begin{array}{rrr}0 . & & \\ 0 & 13 & 17\end{array}$

0.

01419

0.

01719
0415

0.158

9.2383E-02

0514

0 .

068

9.1577E-01

0614

0 .

079

$1.4163 E \quad 00$

0715

0.811

$\begin{array}{rrr}0 . & & 11 \\ 0 & 8 & 17\end{array}$

0.

0416

0.

0

0

0

069

$8 \cdot 3546 E-01$

0615

0.

0710

0.

0716

0.812

0.818

U. 914

0 .

$0.10 \quad 12$

0.1018

0 .

$\begin{array}{lll}0 & 11 & 17\end{array}$

0 .

$\begin{array}{lll}0 & 12 & 17\end{array}$

0.1318

0.

$\begin{array}{lll}0 & 15 & 17\end{array}$

0 .
0.

0915

0.

01013

0.

01019

0.

$0 \quad 1118$

0.

$\begin{array}{lll}0 & 1218\end{array}$

0.

0.1319

0.

- 1518

0.
$2.0127 E-01$

0313

0.

0319

0.

0411

0.

04.17

0510

0516

0.

0610

0616

$\begin{array}{rrr}0 . & & \\ 0 & 7 & 11\end{array}$

0.717

0813

0.
0

0

0916

01014

01113

0.

01119

0.

01219

0.1416

0 .

01519 
$\begin{array}{ccccc}1 & 1 & 3 & 1 & 1 \\ -1.5282 E-01 & -1.0723 E-01 & 0\end{array}$ $\begin{array}{rrrrrr}1 & 1 & 9 & 1 & 1 & 10 \\ 0 & 1 & 1 & 15 & 0 & 1\end{array}$<smiles>[O][Mg]O</smiles>

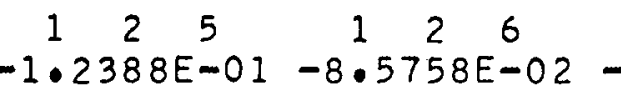

$\begin{array}{ccccccc}1 & 2 & 11 & 1 & 2 & 12 & 0 \\ 0 & & 0 & 0 & & \\ 1 & 2 & 17 & 1 & 2 & 18 & 0 \\ 0 & & 0 & & & 0 \\ 1 & 3 & 8 & 1 & 3 & 9 & \\ -4 & 4932 E-0 & -7 & 1049 E-02 & 0 \\ 1 & 3 & 14 & 1 & 3 & 15 & 1\end{array}$

$\begin{array}{rrrrr}1 & 314 & 1 & 3 & 15 \\ 0 . & & 0 . & & \end{array}$

$\begin{array}{ccccc}1 & 4 & 6 & 1 & 4 \\ 1.5911 E-01 & -1.6181 E-02 & -7\end{array}$

1412

0.418

1413

0.1419

0.511

0.

1517

0.611

0.617

0.712

0.7

$\begin{array}{rrr}1 & 7 & 18 \\ 0 . & & \end{array}$

1814

0.

0 .

$\begin{array}{lll}1 & 9 & 17\end{array}$

0.1015

0.

$\begin{array}{lll}1 & 11 & 14\end{array}$

0.

11214

0 .

11315

0.

$\begin{array}{lll}1 & 14 & 17\end{array}$

0.

$116 \quad 18$

0 .

1512

0.

1518

0.

$\begin{array}{lll}1 & 6 & 12\end{array}$

0

1618

0. 713

0.719

$\begin{array}{rrr}0 . & & \\ 1 & 8 & 15\end{array}$

0.

1912

0.

1918

0.

11016

0.

11115

$\begin{array}{lll}0 . & & \\ 1 & 12 & 15\end{array}$

$\begin{array}{rll}0 . & & \\ 1 & 13 & 16\end{array}$

0 .

$114 \quad 18$

0.

0 .
$-2.8600 E-01-7.7598 E-02$

$116 \quad 19$
1
0
1
0
1

0.

0

$\begin{array}{llll}1 & 2 & 7\end{array}$

$.0026 E-040$.

1213

1219

0.

1310

i 316

0.00

148

$-7.8779 E-02$

1414

0. 57

1513

1
0
1
0
1
0
1
0
1
1

0.

135

$2.2517 E-01$

1311

0.

$13 \quad 17$

0.49

149

.7056E-02

1415

0. 58

$-7.7598 E-0$
1514

0.

1519

0.

1613

168

$8.9924 E-0.2$

1614

0 .

1619

0.714

0.

1

0.

1816

0.

1

913

0.

1919

$0.10 \quad 17$

0.

111.16

0.

11216

0.

$1 \quad 13 \quad 17$

0.

0.

$\begin{array}{lll}1 & 17 & 19\end{array}$

1715

0.

1914

0.

$\begin{array}{lll}1 & 10 & 12\end{array}$

$0.10 \quad 18$

0 .

$\begin{array}{lll}1 & 11 & 17\end{array}$

0.

$\begin{array}{lll}1 & 12 & 17\end{array}$

0. 1318

0 .

$\begin{array}{lll}1 & 15 & 17\end{array}$

0 .
$114 \quad 19$ $\begin{array}{rrrrrr}1 & 1 & 7 & 1 & 1 & 8 \\ 0 . & & & 0 . & & \end{array}$

$1113 \quad 1114$

$0.1119 \quad 0.124$

$0 . \quad 2.2563 E-02$

$\begin{array}{llllll}1 & 2 & 9 & 1 & 2 & 10\end{array}$ $0 . \quad 0$.

$\begin{array}{llllll}1 & 2 & 15 & 1 & 2 & 16\end{array}$

$0 . \quad 0$.

$\begin{array}{llllll}1 & 3 & 6 & 1 & 3 & 7\end{array}$

$4.9026 E-02-2.1497 E-02$

$\begin{array}{llllll}1 & 3 & 12 & 1 & 3 & 13\end{array}$

0.0.

$\begin{array}{llllll}1 & 3 & 18 & 1 & 3 & 19\end{array}$

0.0.

$1410 \quad 1 \quad 411$

0.

1416

0.

0.0

$\begin{array}{llllll}1 & 5 & 9 & 1 & 5 & 10\end{array}$

0

515

0.

1516

0.

$\begin{array}{llllll}1 & 6 & 9 & 1 & 6 & 10\end{array}$

$\begin{array}{lll}1 & 6 & 16\end{array}$

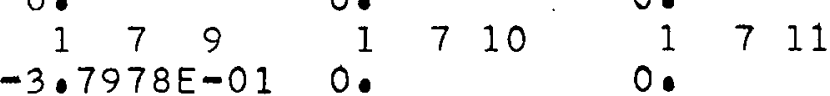

1615

0.

1

$7 \quad 16$

1717

0.0.

1

$8 \quad 12$

$\begin{array}{llll}1 & 8 & 11 & 0 \\ 0 . & & & 0\end{array}$

1

818

0.

1

915

11013

0.

11019

0 .

11118

0.

11218

0.

11319

0.

11518

1813

0.

1819

0.

1916

0.

$110 \quad 14$

0.

11113

0.

11119

0.

11219

0 .

$114 \quad 16$ 
AL $\quad 5.100000 E+00 \quad 1.000000 E+00$

$\begin{array}{ccccc}\text { FORMULA } & \text { DENSITY } & \text { FUEL } & \text { DENSITY } & \text { TEMPERATURE } \\ \text { F } & \text { RHO (G/CC) } & \text { RHOF }(G / C C) & \text { T (DEG K) } \\ 5.1100000 E+00 & 2.7000001 E 00 & 0 . & & 2.9316000 E 02101\end{array}$

PROBABILITY/PATH (/CM)

COLLIDE MULTIPLY $S G(N) \quad N U S G F(N)$

$1.1757 \varepsilon-01$

$1.3114 E-01$

$1.7033 E-01$

$1.6580 E-01$

$1.5978 \varepsilon-01$

0.
0.
0.

$2.5926 E-01$

$2.2459 E-01$

$2.9996 E-01$

$2.8760 E-01$

8.714BE-02

0.

0.

0.

0.

0.

8.1465E-02

0.1586E-02

0.1615E-02

$0.4304 E-02$

8.8920E-02

0.

0.

0.

0.

$9.0583 E-02$

$0.0718 E-02$

$0.3353 E-02$

$1.0125 \mathrm{E}-01$
$0.3 .2784 \mathrm{E}-02$

3. $2226 \mathrm{E}-02$

3. $7423 E-02$

$3.7681 E-02$

4. $7007 E-02$

$6.2639 E-02$

$5.6739 E-02$

$2.5599 E-01$

$2.7711 E-01$

$8.3947 \mathrm{E}=02$

7. $8428 E-02$

$7.8428 E-02$

$7.8138 E-02$

$7.9161 E-02$

$8.0493 E-02$

$8.0773 E-02$

$7.8816 E-02$

$7.8017 E-02$

$0.8949 E-02$
SCATTER

SGSI $N, N) \quad S G S O(N, N+1) \quad S G S I(N, N+1)$

$2.5655 E-02$

$2.7053 E-02$

$3.3506 E-02$

$3.3027 E-02$

$3.5617 E-02$

4. $7644 E-02$

$4.6833 E-02$

$5.4386 E-02$

$5.4761 E-02$

$6.8313 E-02$

$1.7156 E-02$

$2.4929 E-02$

$3.8138 E-02$

$3.5278 E-02$

$1.9465 E-02$

$5.3068 E-02$

$4.6925 E-02$

$2.1424 E-02$

$1.0478 E-02$

$3.1742 E-03$

$9.1031 E-02$

$8.2457 E-02$

$5.0582 E-02$

$8.2457 E-02 \quad 4.0787 E-02$

4.3966E-02 - $1.3912 E-02$

$1.0479 E-02-3.3170 E-03$

$3.1744 E-03-1.0048 E-03$

$2.9655 E-03$

$2.9655 E-03$

$2.9545 E-03$

$3.3513 E-03$

$4 \cdot 1645 E-03$

$2.9657 E-03-9.3879 E-04$

$2.9657 E-03=9.3879 E-04$

$2.9548 E-03-9.3528 E-04$

$4.0422 E-03-1.2795 E-03$

$6.3269 E-03-2.0027 E-03$

$4.1790 E-03$

$4.0776 E-03$

$3.9191 E-03$

$2.2148 E-03$
$6.3489 E-03-2.0097 E-03$

$6.1951 E-03-1.9608 E=03$

$5.7885 E-03-1.8323 E-03$

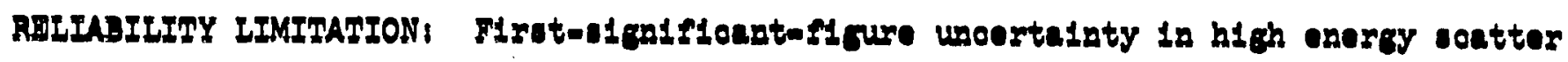
oross seotions of aluminum. 


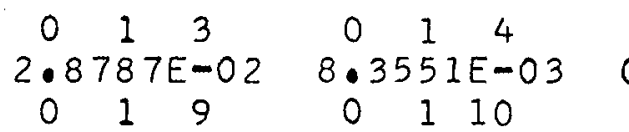
0 . 0115

0.

025

$1.7229 \mathrm{E}-02$ 0211

0.

0 .

038

$7.3603 E-03$

0314

0 .

046

$3.3214 E-02$

0412

0 .

0418

0.011

0.

0.

0

0.

0 .

0712

0.718

0 .

0

0

0.

0 .

$0 \quad 1015$

0.11114

0 .

$0 \quad 1214$

0.1315

0 .

$0 \quad 1417$

$\begin{array}{lll}0.16 \quad 18 & \end{array}$

0.
0 .

0116

0.

026

$6.4342 E-03$

0212

$0:$

218

0 .

039

$6.0265 E-03$

0315

0.

047

$2.0145 E-02$

0413

0.
0 419

0 .

$0 \quad 512$

0.

$0 \quad 518$

$\begin{array}{lll}0 & 0 & 12\end{array}$

0 .

$0 \quad 618$

0 .

0713

$0: 019$

$0: 015$

0 .

0912

0 .

$\begin{array}{lll}0 & 9 & 18\end{array}$

0.010
0

$\begin{array}{lll}0 & 1115\end{array}$

0.01215

$\begin{array}{lll}0.0 & 13 & 16\end{array}$

0.

01418

$\begin{array}{lll}0 & 16 \quad 19\end{array}$

0.
0
0
0

0.

117

$\begin{array}{lll}0 & 0 & 1 \\ 0 & 2 & 17\end{array}$

6.0693E-06

02.13

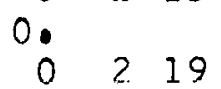

0 .

0310

0.

0316

0.48

$1.2219 E-02$

0414

0 .

057

3.8883E-02

0513

$\begin{array}{lll}0 & 519\end{array}$

$\begin{array}{lll}0 . & & \\ 0 & 6 & 13\end{array}$

$\begin{array}{lll}0 & 0 & 19 \\ 0 & 6 & 19\end{array}$

0.

0714

$\begin{array}{lll}0 & 8 & 10\end{array}$

$\begin{array}{lll}0.8 & 8 & 16\end{array}$

0.

$0 \quad 913$

0 .

$\begin{array}{lll}0 & 1 & 6 \\ 0 & 1 & 12\end{array}$

$0 \quad 112$

0.18

$\begin{array}{rrr}0 & 0 & \\ 0 & 2 & \end{array}$

0.

0214

$0: 35$

3.2987E-02

0311

0.

0.017

0.049

$7.7818 \mathrm{E}-03$

0415

0 .

058

5.5700E-03

$0 \quad 514$

0.

068

0614

$0 .: 79$

$8.5392 E-02$

0715

$0: 011$

$\begin{array}{lll}0 & 0 & 17\end{array}$

0.

$0 \bigcirc 14$

0.1012

$0 \quad 919$

$\begin{array}{lll}0 & 10 & 17\end{array}$

0 .

01018

0.11116

0.

$0 \quad 1117$

0.1216

$0.012 \quad 17$

0 .

$\begin{array}{lll}0 & 13 & 17\end{array}$

0 .

01318

0 .

01419

$0.015 \quad 17$

0 .

$0 \quad 1719$

of

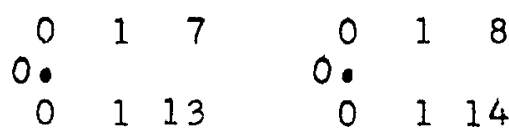

$\begin{array}{llllll}0 & 0 & 19 & 0 & & \\ 0 & 1 & 19 & 4\end{array}$

0. $2.8406 E-02$

$\begin{array}{lllll}0 & 29 & 0 & 2 & 10\end{array}$

$\begin{array}{cccccc}0.0 & & 0.0 & & \\ 0 & 2 & 15 & 0 & 2 & 16\end{array}$

$\begin{array}{lllll}0 . & & 0 & & \\ 0 & 3 & 6 & 0 & 37\end{array}$

$2.0007 E-02 \quad 1.2135 E-02$.

$\begin{array}{lllllll}0 & 3 & 12 & 0 & 3 & 13\end{array}$

0.

0.

318

0.

0319

0

410

0 .

411

$\begin{array}{llllll}0 & 4 & 16 & 0 & 4 & 17\end{array}$

0 .

059

0 .

$0 \quad 510$

0.

$0 \quad 515$

0516

$\begin{array}{llllll}0 & 0 & 0 & 0 & 0 & 6\end{array}$

5.0371E-02 O.

0615

0616

$0: 710$

0.711

0.716

0 .

$0: 012$

0717

0

$\begin{array}{lll}0 & 8 & 13\end{array}$

0.818

0.

0 .

$0 \quad 8 \quad 19$

0915

0 .

0916

0.

01013

$0 \quad 1014$

0.

01019

0 .

$\begin{array}{lll}0 & 11 & \\ 0 & 11 & 18\end{array}$

01113

0.01118

$0 \quad 1218$

01119

0 .

$\begin{array}{lll}0 & 13 & 19\end{array}$

0 .

$0 \quad 1518$

01219

0.01416

0 .

$0 \quad 1519$ 


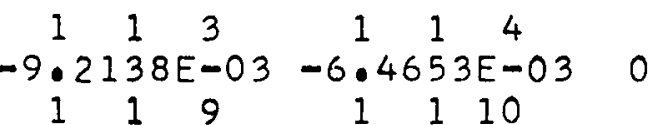

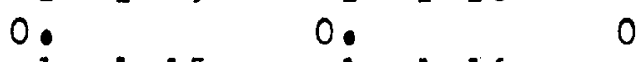

1115 i 116

$\begin{array}{llllll}1 & 2 & 5 & 1 & 2 & 6\end{array}$

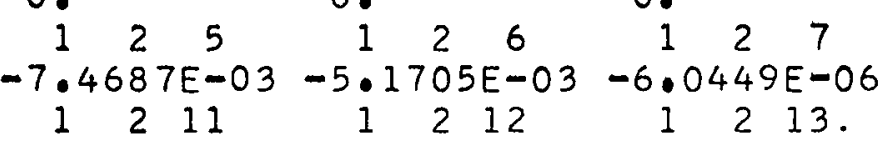

0.

1

1212

0.

170

1218

0.

38

0.

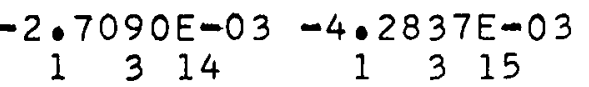

0

146

0.

147

1412

0.

1

418

0.

1

0.

511

$5 \quad 17$

0.

1

0

0 .

0

0

0

0

0.

0.

0

0.

1

0.

1

0.

1

0.

1

11417

$n$.

11618

0.

1513

1519

0.613

0.

1619

0.

1714

0.810

1
1

11116

11115

0.

11216

11215

0

0.

11418

$114 \quad 19$

11619

11719
1213 .
1

.

1117

0.

1

0.

11
150

219

310

1316

0.

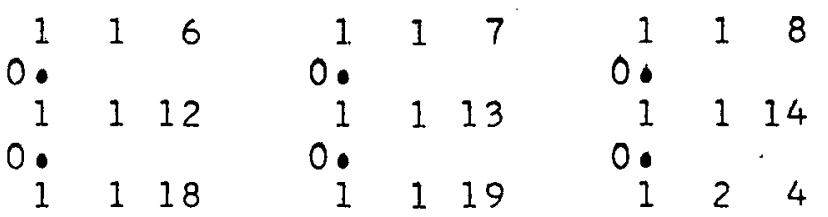

$0 . \quad 0.1 .3604 \mathrm{E}-03$

$\begin{array}{lllllllll}1 & 2 & 8 & 1 & 2 & 9 & 1 & 2 & 10\end{array}$

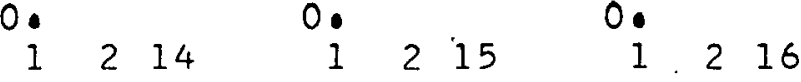

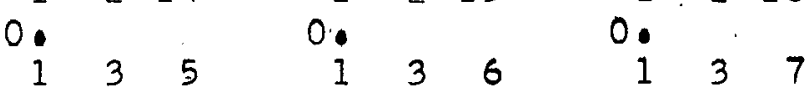

$1.3576 E-02 \quad 2.9559 E-03-1.2961 E-03$

$\begin{array}{lllllllll}1 & 3 & 11 & 1 & 3 & 12 & 1 & 3 & 13\end{array}$

$\begin{array}{rrrrrrr}0.17 & 0 & 1 & 3 & 0 . & 18 & 1\end{array}$

$0 . \quad 0$

$149 \quad 1$

4. $10 \quad 0: 1411$

$-4.7497 E-03-5.8517 E-03 \quad 0$.

1415

1416

0.

$\begin{array}{lll}1 & 4 & 17\end{array}$

0.

0.

9

I $5 \quad 8$

$-1.7243 E-02-4.6785 E-03$

0

1510

0.

1

0 .

515

169

5.8

1614

0.

1615

0. 516

1516

1610

179

$-2.2898 E-02$

0.

1710

0.

0 .

0

715

1

716

0.

812

0.

1

811

1

$8 \quad 17$

0.

818

0.

1

9.14

0.

11012

0.

1915

0.

I 1013

0.

11018

0.

i 1019

0.

$\begin{array}{lll}1 & 11 & 17\end{array}$

0.

11118

0.

$\begin{array}{lll}1 & 12 & 17\end{array}$

0.

11218

0.

11318

0.

11319

11517

0.

11518

0.
1616

0.711

0.717

$\begin{array}{lll}1 & 7 & 17\end{array}$

0.813

0.819

0

1916

0.

$110 \quad 14$

0.1113

0.

11119

0.

$1 \quad 1219$

0.

$114 \quad 16$

0.

11519
0.

0 . 
ATOM

CODE

MASS FRACTION

ATOM

CODE

-

$5.500000 E+00 \quad 1.000000 E+00$

FORMULA

$F$

$5.5900000 E+00$

\author{
DENSITY \\ RHO $(G / C C)$
}

$\begin{array}{cl}\text { FUEL } & \text { DENSITY } \\ \text { RHOF } & (G / C C)\end{array}$

0.
TEMPERATURE

$T$ (DEG K)

$2.3300010 E$ OO

$2.9316000 E \quad 02$

PROBABILITY/PATH (/CM)

$\begin{array}{cc}\text { COLLIDE } & \text { MULT } \\ \text { SG(N) } & \text { NUS } \\ 9.2951 E-02 & 0 . \\ 1.0819 E-01 & 0 . \\ 1.3218 E-01 & 0 . \\ 1.6441 E-01 & 0 . \\ 1.7491 E-01 & 0 . \\ 1.6516 E-01 & 0 . \\ 1.7616 E-01 & 0 . \\ 1.8091 E-01 & 0 . \\ 1.2619 E-01 & 0 . \\ 9.1214 E-02 & 0 . \\ 1.0123 E-01 & 0 . \\ 1.0503 E-01 & 0 . \\ 1.0969 E-01 & 0 . \\ 1.1196 E-01 & 0 . \\ 1.1217 E-01 & 0 . \\ 1.1231 E-01 & 0 . \\ 1.1237 E-01 & 0 . \\ 1.1219 E-01 & 0 . \\ 1.1171 E-01 & 0 .\end{array}$

MULT IPLY

$\operatorname{NUSGF}(N)$

\begin{abstract}
SGSO $(N, N)$
7. $9882 E-02$

$9.2981 E-02$

$1.1360 E-01$

$1.4130 E-01$

$1.5031 \mathrm{E}-01$

$1.4194 \mathrm{E}-01$

$1.5139 E-01$

$1.5547 \mathrm{E}-01$

$1.2175 E-01$

$8.7996 E-02$

9.7640E-02

1.0126E-01

$1.0560 E-01$

$1.0622 E-01$

$1.0337 \mathrm{E}-01$

$1.0291 E-01$

$1.0198 E-01$

$1.0056 E-01$

$1.0594 E-01$
\end{abstract}

SCATTER

$\operatorname{SGSI}(N, N) \quad \operatorname{SGSO}(N, N+1) \quad \operatorname{SGSI}(N, N+1)$

$6.3759 E-03$

$7.4217 E-03$

$9.0671 E-03$

$1.1277 \mathrm{E}-02$

1.1999E-02

$1.3069 E-02-4.1450 E-03$

$1.5212 E-02-4.8250 E-03$

I. $8585 E-02-5.8948 E-03$

$2.3117 E-02-7.3311 E-03$

$2.4593 E-02-7.8007 E-03$

$1.1329 E-02$

$1.2083 E-02$

1.2408E-02

$4.4353 E-03$

$3.2057 E-03$

$2.3223 E-02-7.3654 E-03$

$2.4768 E-02-7.8548 E-03$

$2.5436 E-02-8.0662 E-03$

4.4355E-03-1.4069E-03

$3.2059 E-03-1.0169 E-03$

$3.5569 E-03$

$3.6887 E-03$

$3.8468 E-03$

4. $3313 E-03$

$5.1476 E-03$

$3.5572 E-03-1.1282 E-03$

$3.6889 E-03-1.1700 E-03$

$3.8470 E-03-1.2202 E-03$

$5.2231 E-03-1.6567 E-03$

$7.8170 E-03-2.4790 E-03$

$5.1245 E-03$

$5.0784 E-03$

4. $8539 E-03$

$2.5321 E-03$
$7.7819 E-03-2.4679 E-03$

$7.7116 E-03-2.4458 E-03$

$7.1661 E-03-2.2729 E-03$ 


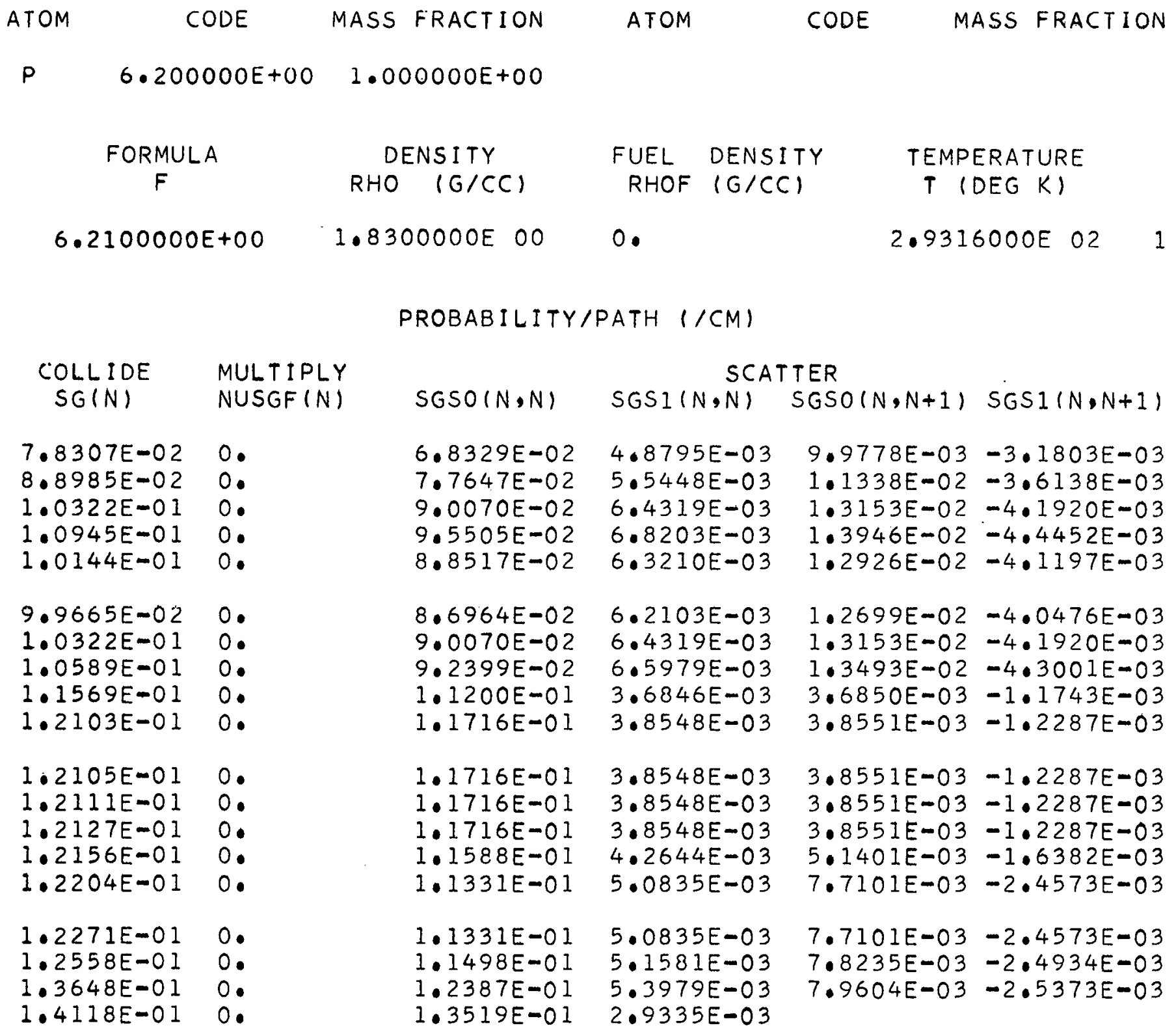


ATOM

CODE MASS FRACTION

ATOM

CODE

MASS FRACTION

$S$

$6 \cdot 100000 E+00 \quad 1.000000 E+00$

FORMULA

$F$

$6.1100000 E+00$
DENSITY

RHO (G/CC)

$1.8110003 \mathrm{E} 00$
FUEL DENSITY RHOF (G/CC)
TEMPERATURE

$T$ (DEG K)

$2.9316000 E \quad 02 \quad 1$

PROBABILITY/PATH (/CM)

$\begin{array}{cc}\text { COLLIDE } & \text { MULT } \\ \text { SUIN } & \text { NUS } \\ 7.4007 E-02 & 0 . \\ 9.1870 E-02 & 0 . \\ 1.0072 E-01 & 0 . \\ 9.0067 E-02 & 0 . \\ 7.3566 E-02 & 0 . \\ 9.1023 E-02 & 0 . \\ 8.2517 E-02 & 0 . \\ 1.1059 E-01 & 0 . \\ 1.0209 E-01 & 0 . \\ 3.4057 E-02 & 0 . \\ 3.4111 E-02 & 0 . \\ 3.4257 E-02 & 0 . \\ 3.4655 E-02 & 0 . \\ 3.5459 E-02 & 0 . \\ 3.7285 E-02 & 0 . \\ 3.9639 E-02 & 0 . \\ 4.2396 E-02 & 0 . \\ 4.9012 E-02 & 0 . \\ 5.0944 E-02 & 0 .\end{array}$

SCATTER

SGSO $(N, N) \quad \operatorname{SGSI}(N, N) \quad \operatorname{SGSO}(N, N+1) \quad \operatorname{SGSI}(N, N+1)$

$5.2944 E-02$

$6.8603 E-02$

$3.6469 E-03$

$7.4525 E-03-2.3786 E-03$

$8.0534 \mathrm{E}-02$

$7.6805 E-02$

$6.4129 E-02$

4.7253E-03

$9.6568 E-03-3.0819 E-03$

$5.5476 E-03$

$5.2912 E-03$

4.4174E-03

$1.1336 E-02-3.6183 E-03$

$1.0811 E-02-3.4513 E-03$

$9.0270 E-03-2.8812 E-03$

$7.9788 E-02$

$5.4960 E-03$

1. $1231 E-02-3.5846 E-03$

$7.2331 E-02$

$9.6939 E-02$

$9.8929 E-02$

$4.9827 E-03$

$6.6775 \mathrm{E}-03$

$1.0182 E-02-3.2499 E-03$

$1.3646 E-02-4.3552 E-03$

$3.1487 E-03$

$3.1490 E-03-1.0050 E-03$

$3.2976 E-02$

$1.0496 E-03$

$1.0497 E-03-3.3501 E-04$

$3.2976 E-02$

$3.2976 E-02$

$3.2976 E-02$

3. 2626E-02

$1.0496 E-03$

I. $0496 E-03$

$1.0497 E-03-3.3501 E-04$

$1.0496 E-03$

$1.0497 E-03-3.3501 E-04$

$1.0497 E-03-3.3501 E-04$

$1 \cdot 1612 E-03$

$1.4123 E-03$

$1.3995 E-03-4.4668 E-04$

$2.1413 E-03-6.8343 E-04$

$3.3204 E-02$

3. $3204 E-02$

$1.4400 E-03$

$2.1833 E-03-6.9685 E-04$

$1.4400 E-03$

$2.1833 E-03-6.9685 E-04$

$3.4884 E-02$

$1.4696 \mathrm{E}-03$

$2.1666 E-03-6.9155 E-04$

$7.5928 E-04$ 


\begin{tabular}{|c|c|c|c|c|c|}
\hline \multicolumn{2}{|c|}{ CODE } & MASS FRACTION & \multirow[t]{2}{*}{ ATOM } & CODE & MASS FRACTION \\
\hline \multicolumn{2}{|c|}{$7.400000 E+00$} & $1 \cdot 0$ & & & \\
\hline \multicolumn{2}{|c|}{$\begin{array}{c}\text { FORMULA } \\
\text { F }\end{array}$} & $\begin{array}{l}\text { DENSITY } \\
\text { to } 1 G / C C\end{array}$ & $\begin{array}{ll}\text { FUEL } & \text { DENSI } \\
\cdot \text { RHOF } & \mid G / C\end{array}$ & $\begin{array}{l}\text { DENSITY } \\
(G / C C)\end{array}$ & TEMPERATURE \\
\hline \multicolumn{2}{|c|}{$7.4100000 E+00$} & $8.6999990 E-01$ & $0 !$ & \multicolumn{2}{|c|}{$2.9316000 \mathrm{E} .02$} \\
\hline \multicolumn{6}{|c|}{ PROBABILITY/PATH (/CM) } \\
\hline $\begin{array}{l}\text { COLLIDE } \\
\text { SG(N) }\end{array}$ & $\begin{array}{l}\text { MULTIPL } \\
\text { NUSGFIN }\end{array}$ & $\operatorname{SGSO}(N, N)$ & $\operatorname{SGSI}(N, N)$ & $\begin{array}{l}\text { TTER } \\
\text { SGSO }(N, N+1)\end{array}$ & $\operatorname{SGSI}(N, N+1)$ \\
\hline $\begin{array}{l}3.7258 E-02 \\
4.2954 E-02 \\
4.4898 E-02 \\
4.2084 E-02 \\
3.5719 E-02\end{array}$ & $\begin{array}{l}0 \\
0 \\
0 \\
0 \\
0\end{array}$ & $\begin{array}{l}3.3477 E-02 \\
3.8595 E-02 \\
4.0341 E-02 \\
3.7812 E-02 \\
3.2092 E-02\end{array}$ & $\begin{array}{l}1.8575 E-03 \\
2.1415 E-03 \\
2.2379 E-03 \\
2.0973 E-03 \\
1.7803 E-03\end{array}$ & $\begin{array}{l}3.7796 E-03 \\
4.3574 E-03 \\
4.5545 E-03 \\
4.2690 E-03 \\
3.6232 E-03\end{array}$ & $\begin{array}{l}-1.2167 E-03 \\
-1.4027 E-03 \\
-1.4657 E-03 \\
-1.3735 E-03 \\
-1.1660 E-03\end{array}$ \\
\hline $\begin{array}{l}2.9355 E-02 \\
2.5336 E-02 \\
2.7482 E-02 \\
2.5481 E-02 \\
2.0286 E-02\end{array}$ & $\begin{array}{l}0 . \\
0 . \\
0 . \\
0 . \\
0 .\end{array}$ & $\begin{array}{l}2.6372 E-02 \\
2.2759 E-02 \\
2.4686 E-02 \\
2.4817 E-02 \\
1.9723 E-02\end{array}$ & $\begin{array}{l}1.4630 E-03 \\
1.2626 E-03 \\
1.3692 E-03 \\
6.4572 E-04 \\
5.1324 E-04\end{array}$ & $\begin{array}{l}2.9774 E-03 \\
2.5696 E-03 \\
2.7871 E-03 \\
6.4579 E-04 \\
5.1323 E-04\end{array}$ & $\begin{array}{l}-9.5814 E-04 \\
-8.2697 E-04 \\
-8.9667 E-04 \\
-2.0775 E-04 \\
-1.6517 E-04\end{array}$ \\
\hline $\begin{array}{l}2.1510 E-02 \\
2.3953 E-02 \\
2.7061 E-02 \\
2.9503 E-02 \\
3.1470 E-02\end{array}$ & $\begin{array}{l}0 . \\
0 . \\
0 . \\
0 . \\
0 .\end{array}$ & $\begin{array}{l}2.0833 E-02 \\
2.2989 E-02 \\
2.5405 E-02 \\
2.6480 E-02 \\
2.6080 E-02\end{array}$ & $\begin{array}{l}5.4210 E-04 \\
5.9818 E-04 \\
6.6114 E-04 \\
7.6966 E-04 \\
9.2088 E-04\end{array}$ & $\begin{array}{l}5.4212 E-04 \\
5.9821 E-04 \\
6.6109 E-04 \\
9.2677 E-04 \\
1.3935 E-03\end{array}$ & $\begin{array}{l}-1.7444 E-04 \\
-1.9248 E-04 \\
-2.1281 E-04 \\
-2.9827 E-04 \\
-4.4834 E-04\end{array}$ \\
\hline $\begin{array}{l}3.4398 E-02 \\
3.9678 E-02 \\
4.8480 E-02 \\
5.3723 E-02\end{array}$ & $\begin{array}{l}0 . \\
0 . \\
0 . \\
0 .\end{array}$ & $\begin{array}{l}2.6398 E-02 \\
2.7352 E-02 \\
2.8846 E-02 \\
3.0308 E-02\end{array}$ & $\begin{array}{l}9.3207 E-04 \\
9.6600 E-04 \\
9.9006 E-04 \\
5.2129 E-04\end{array}$ & $\begin{array}{l}1.4105 E-03 \\
1.4615 E-03 \\
1.4568 E-03\end{array}$ & $\begin{array}{l}-4.5377 E-04 \\
-4.7041 E-04 \\
-4.6886 E-04\end{array}$ \\
\hline
\end{tabular}




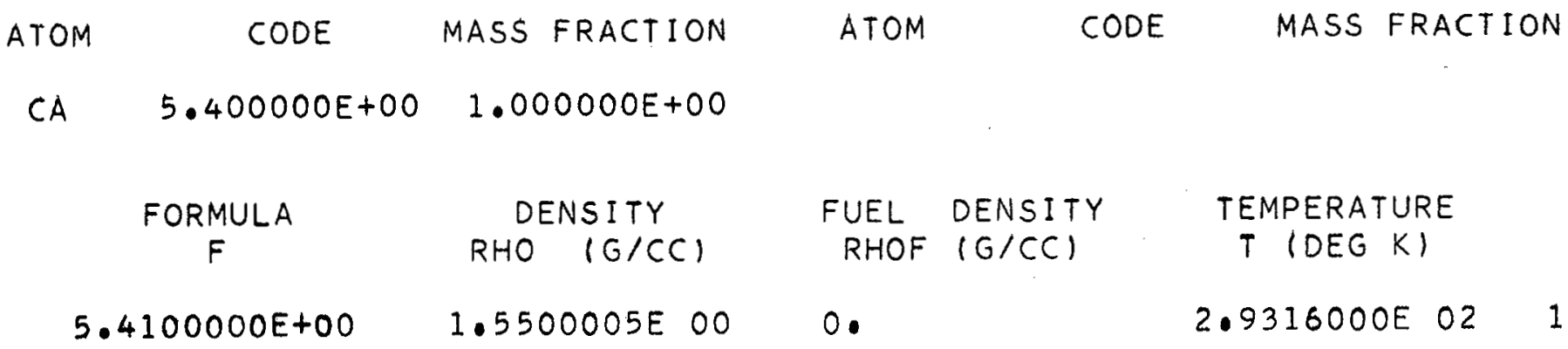

PROBABILITY/PATH (/CM)

\section{COLLIDE MULTIPLY $S G(N) \quad N U S G F(N)$}

$6.9898 E-02$

$6.9898 E-02$

$6.9899 E-02$

$6.9899 E-02$

$6.9899 E-02$

$6.9900 E-02$

$6.9900 E-02$

$6.9901 E-02$

$6.9905 E-02$

$6.9916 E-02$

$6.9949 E-02$

$7.0037 E-02$

$7.0275 E-02$

$7.0693 E-02$

$7.1414 E-02$

$7.2398 E-02$

$7.4020 E-02$

$7.6796 E-02$

$7.8783 \mathrm{E}-02$
0.
0 .
0.
0.
0.
0.
0.
0.
0.
0.
0.
0.
0.
0.
0.
0.
0 .
0.

SCATTER

SGSO $(N, N) \quad S G S I(N, N)$ SGSO $(N, N+1) \quad S G S I(N+N+1)$

$6.2969 E-02$
$6.2969 E-02$
$6.2969 E-02$
$6.2969 E-02$
$6.2969 E-02$

$3.4059 E-03$

$3.4059 E-03$

$3.4059 E-03$

$3.4059 E-03$

$3.4059 E-03$

$6.9288 E-03-2.2317 E-03$

$6.9288 E-03-2.2317 E-03$

$6.9288 E-03-2.2317 E-03$

$6.9288 E-03-2.2317 E-03$

$6.9288 E-03-2.2317 E-03$

$6.2969 E-02$

$6.2969 E-02$

$3.4059 E-03$

$3.4059 E-03$

$3.4059 E-03$

$6.2969 E-02$

$6.8165 E-02$

$1.7322 E-03$

$6.9288 E-03-2.2317 E-03$

$6.9288 E-03-2.2317 E-03$

$6.9288 E-03-2.2317 E-03$

$1.7322 E-03-5.5792 E-04$

$1.7322 E-03-5.5792 E-04$

$6.8165 E-02$

$1.7322 E-03$

$1.7322 E-03-5.5792 E-04$

$6.8165 E-02$

$6.8165 E-02$

$6.7588 E-02$

$1.7322 \mathrm{E}-03$

$1.7322 \mathrm{E}-03$

$1.7322 E-03-5.5792 E-04$

$1.7322 E-03-5.5792 E-04$

$1.9182 E-03$

$2.3096 E-03-7.4389 E-04$

$3.4644 E-03-1.1158 E-03$

$6.6433 E-02$

$6.6433 E-02$

$6.6614 E-02$

$2.2901 E-03$

$\begin{array}{ll}2.2901 E-03 & 3.4644 E-03-1.1158 E-03 \\ 2.2901 E-03 & 3.4644 E-03-1.1158 E-03\end{array}$

$2.2319 E-03 \quad 3.2836 E-03-1.0576 E-03$ 


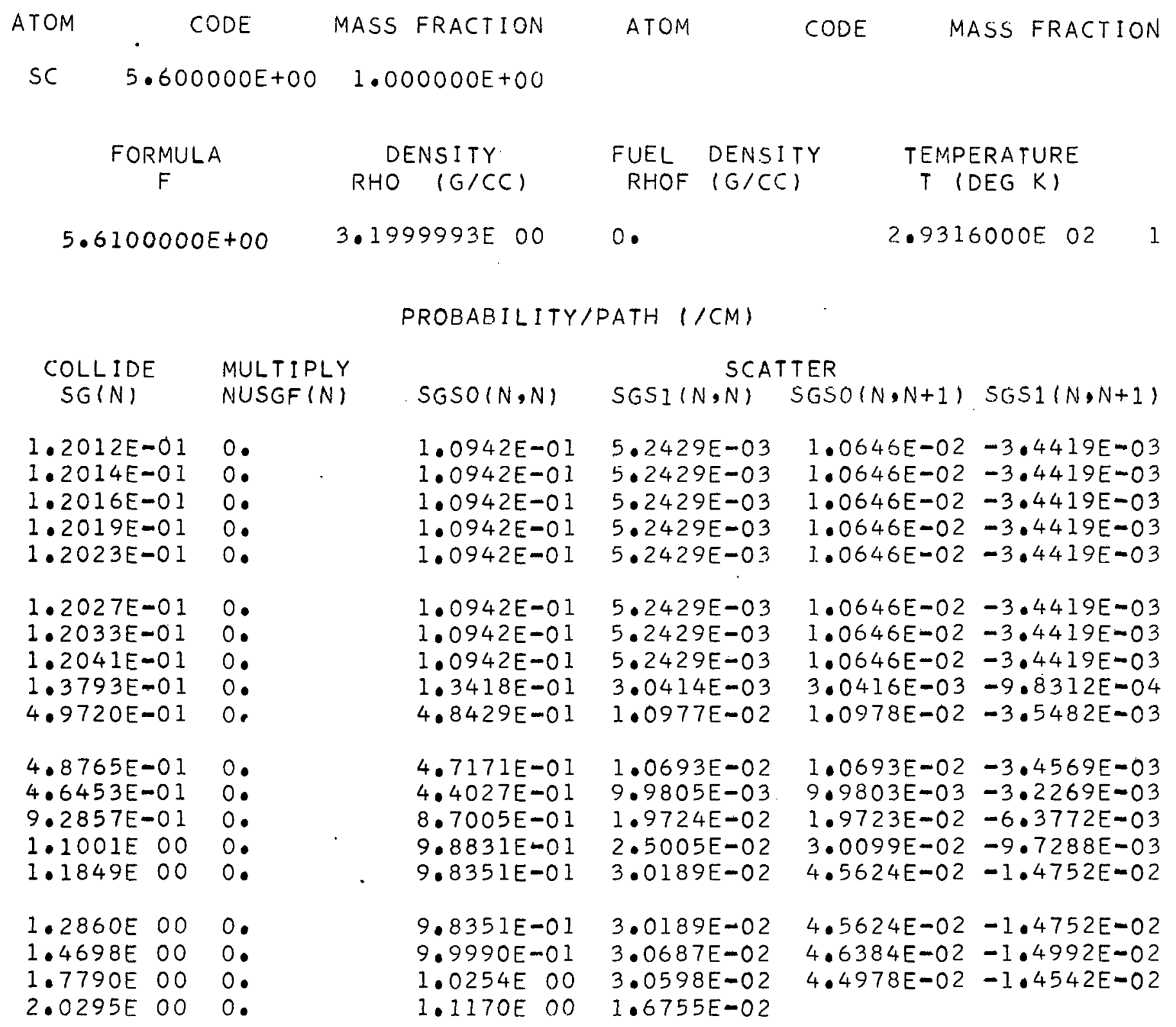




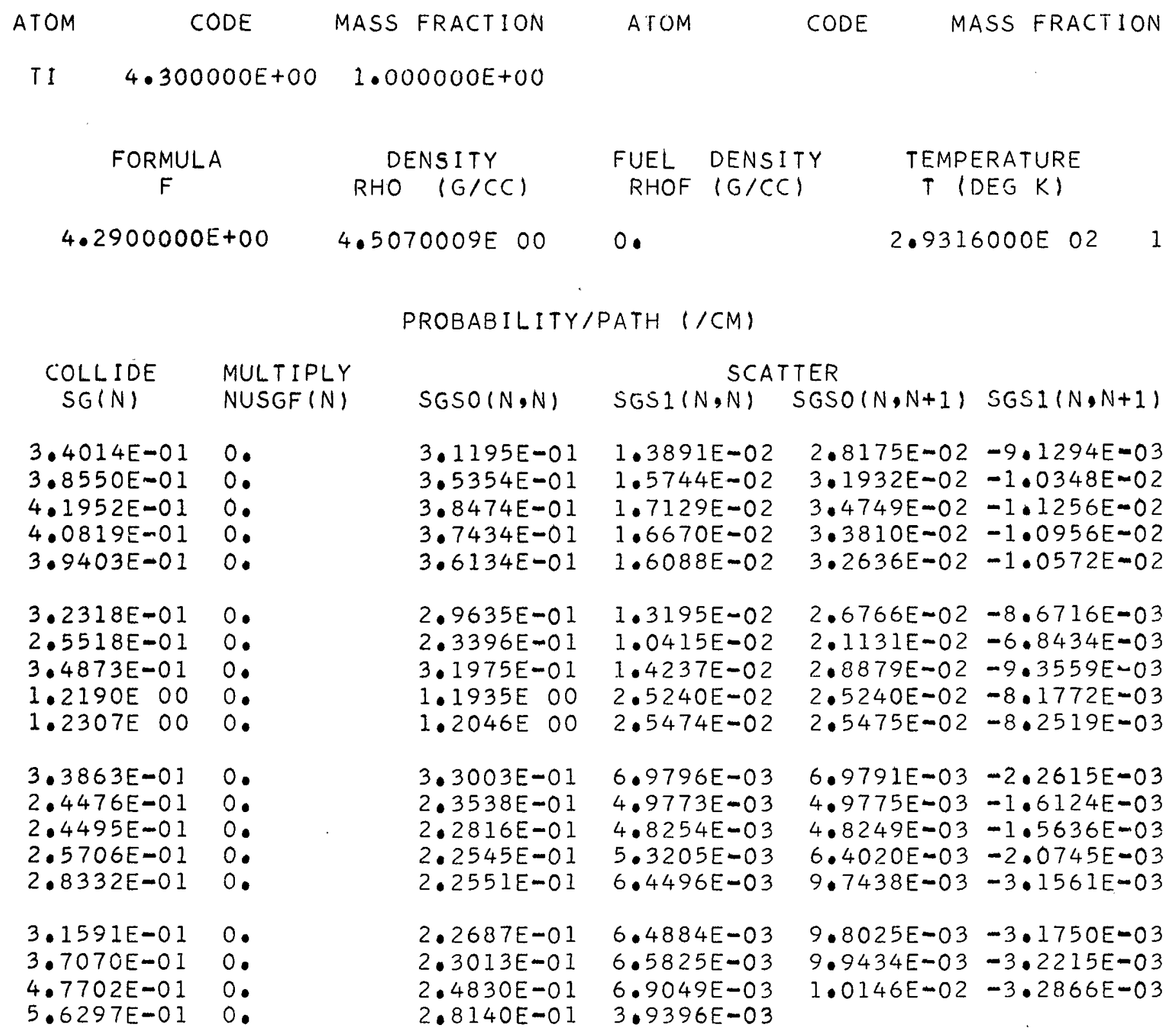




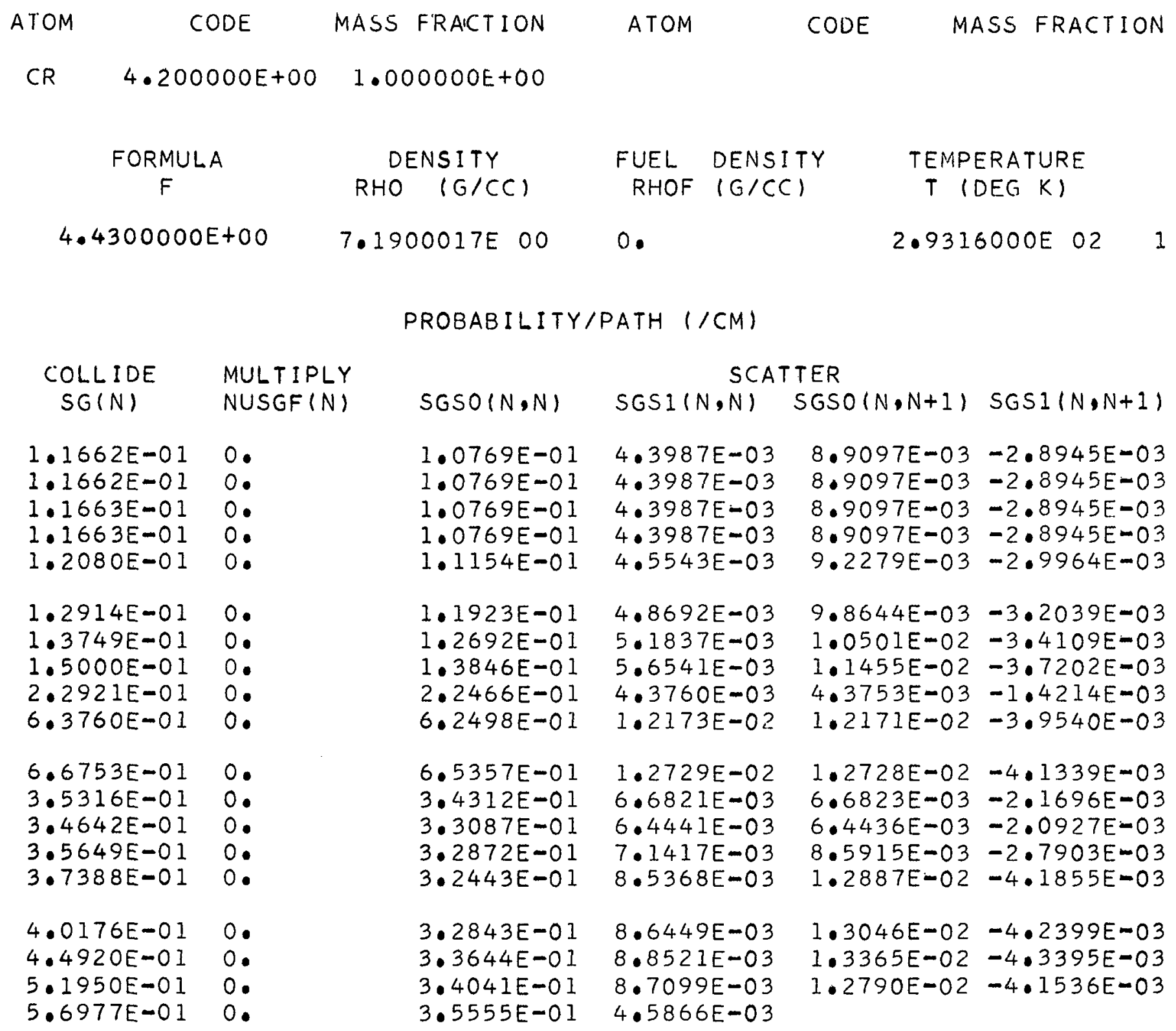


ATOM

COUE

MASS IRACTION

ATOM

CODE

MASS FRACIION

MN $\quad 4.400000 E+00 \quad 1.000000 E+00$

FORMULA

$F$

$4 \cdot 3900000 E+00$
DENSITY

RHO (G/CC)
FUEL DENSITY RHOF $(G / C C)$
TEMPERATURE

$T$ (DEG K)

$2.9316000 E 02$

PROBABILITY/PATH (/CM)

$\begin{array}{cc}\text { COLLIDE } & M U \\ \text { SGIN) } & N U \\ 2.7264 E-01 & 0 . \\ 3.0114 E-01 & 0 . \\ 3.0116 E-01 & 0 . \\ 2.8899 E-01 & 0 . \\ 2.7478 E-01 & 0 . \\ 2.6466 E-01 & 0 . \\ 2.9117 E-01 & 0 . \\ 3.1769 E-01 & 0 . \\ 5.8659 E-01 & 0 . \\ 8.8914 E-00 & 0 . \\ 1.0959 E 01 & 0 . \\ 2.5821 E 00 & 0 . \\ 2.5693 E-01 & 0 . \\ 2.6470 E-01 & 0 . \\ 3.4162 E-01 & 0 . \\ & \\ 4.6215 E-01 & 0 . \\ 6.6614 E-01 & 0 . \\ 9.7125 E-01 & 0 . \\ 1.1614 E-00 & 0 .\end{array}$

$7.4199984 \mathrm{E} 00$

0. .

MULTIPLY $\operatorname{NUSGF}(N)$

0.

0 .

0 .

0.

0 .

0 .

0.

0.

0.

0.

0 .

0.

0.

0 .

0.

0.

0

\section{$\operatorname{SGSO}(N, N)$}

2.5287E-01

2.7929E-01

$2.7929 E-01$

$2.6797 E-01$

$2.5476 E-0 I$

$2.4532 E-01$

2.6985E-01

2. $9439 E-01$

$5.7526 E-01$

$8.7287 E 00$

1.0706E 01

2.5207E 00

2. $1253 E-01$

I. $7509 \mathrm{E}-01$

$1.7254 \mathrm{E}-01$

$1.8705 E-01$

2.1606E-0I

2. $2381 E-01$

2. $0855 E-01$
SCATTER

$\operatorname{SCSI}(N, N) \quad S G S O(N, N+1) \quad \operatorname{SGS} 1(N, N+1)$

$9.7340 E-03$

$1.0758 E-02$

$1.0758 E-02$

$1.0318 E-02$

$1.9712 E-02-6.4085 E-03$

$2.1771 E-02-7.0847 E-03$

$2.1771 E-02-7.0847 E-03$

$2.0889 E-02-6.7936 E-03$

$1.9859 E-02-6.4622 E-03$

$9.4493 E-03$

$1.0388 E-02$

$1.1334 E-02$

$1.0593 E-02$

$1.9123 E-02-6.2231 E-03$

2. $.1036 E-02-6.8395 E-03$

$2.2948 E-02-7.4625 E-03$

$1.0591 E-02-3.4459 E-03$

$1.6071 E-01-5.22 .60 E-02$

$1.9714 E-01 \quad 1.9712 E-01-6.4119 E-02$

4.6421E-0? 4.6411E-02 - $1.5101 E-02$

$3.9130 E-03 \quad 3.9129 E-03-1.2724 E-03$

$3.5949 E-03 \quad 4.3248 E-03-1.4060 E-03$

$4.2894 E-03 \quad 6.4725 E-03-2.1055 E-03$

4.6495E-03 7.0168E-03-2.2819E-03

$5.3721 E-03 \quad 8.1054 E-03-2.6373 E-03$

$5.4123 \mathrm{E}-03 \quad 7.9423 \mathrm{E}-03-2.5849 \mathrm{E}-03$

$2 \cdot 5443 E-03$ 


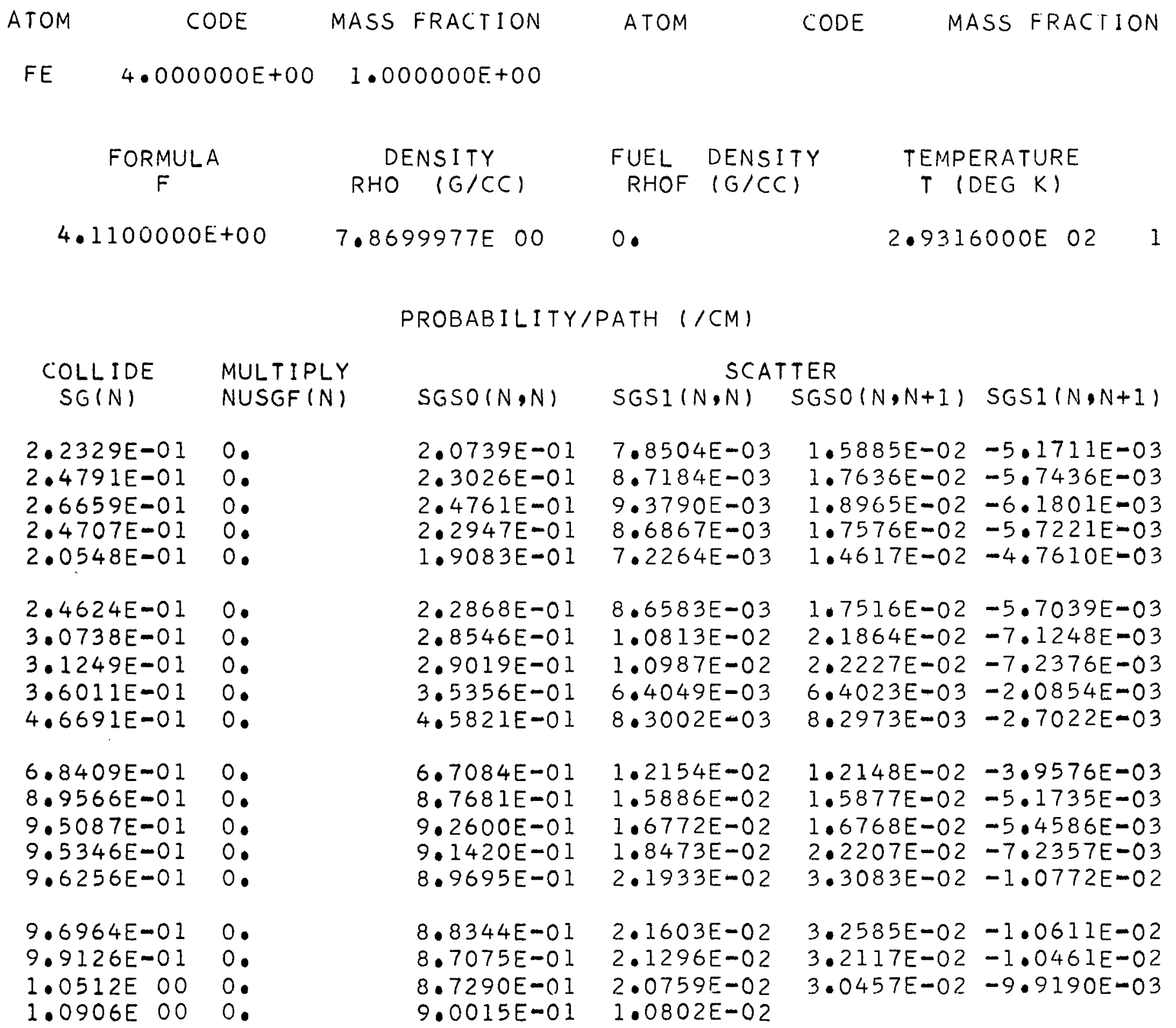


ATOM

CODE

MASS FRACTION

ATOM

CODE

MASS FRACIION

NI $\quad 4.100000 E+00 \quad 1.000000 E+00$

FORMULA

$F$

$4 \cdot 4100000 E+00$

\author{
DENSITY \\ RHO (G/CC)
}

$8.8999976 E \quad 00$
FUEL DENSITY
RHOF (G/CC)

0.
TEMPERATURE

$T$ (DEG K)

$2.9316000 E^{02} 1$

PROBABILITY/PATH (/CM)

\section{COLLIDE $S G(N)$}

$2.5584 E-01$

$2.7412 E-01$

2.8326E-01

$2.7870 E-01$

2. $9242 E-01$

$3.2899 E-01$

4.2037E-01

$5.1633 E-01$

8.2254E-01

1. $3758 \mathrm{E} 00$

$1.7015 \mathrm{E} \quad 00$

$1.7691 \mathrm{E} \quad 00$

$1.7974 E \quad 00$

$1.8058 \mathrm{E} 00$

$1.7949 \mathrm{E} 00$

$1.8134 \mathrm{E} \quad 00$

$1.7626 \mathrm{E} 00$

$1.7789 \mathrm{E} \quad 00$

$1.9093 E \quad 00$
MULTIPLY

$\operatorname{NUSGF}(N)$
0 .

0.

0 .

0.

0 .

0.

0.

0 .

0 .

0.

0.

0.

0.

0 .

0.

0.

0 .

0 .

0.

\section{$\operatorname{SGSO}(N, N)$}

$2.3836 E-01$

$2.5539 E-01$

$2.6390 E-01$

$2.5964 E-01$

2.7241E-01

$3.0647 E-01$

3. $9159 E-01$

4. $8098 E-01$

8.0823E-01

1. $3515 E 00$

1.6703E 00

$1.7332 \mathrm{E} \quad 00$

$1.7512 \mathrm{E} \quad 00$

$1.7321 E \quad 00$

$1.6722 \mathrm{E} 00$

$1.6502 \mathrm{E} 00$

$1.5355 E$ OO

$1.4414 \mathrm{E} 00$

$1.5366 E \quad 00$
SCATTER

SGSI $N, N) \quad$ SGSO $(N, N+1) \quad S G S I(N, N+1)$

$8.6282 E-03 \quad 1.7450 E-02-5.6863 E-03$

$9.2404 E-03 \quad 1.8696 E-02-6.0885 E-03$

$9.5481 E-03 \quad 1.9319 E-02-6.2910 F-0$ ?

$9.3984 E-03 \quad 1.9008 E-02-6.1939 E-03$

$9.8708 E-03 \quad 1.9943 E-02-6.5087 E-03$

1.1098E-02 2.2436E-02 -7.3156E-03

$1.4176 E-02 \quad 2.8668 E-02-9.3433 E-03$

$1.7414 \mathrm{E}-02 \quad 3.5211 \mathrm{E}-02-1.1478 \mathrm{E}-0$ ?

$1.4023 E-02 \quad 1.402 .2 E-02-4.5666 E-03$

$2.3449 E-02 \quad 2.3448 E-02-7.6364 E-03$

2.8979E-02

$3.0080 E-0$ ?

$3.0396 E-02$

$3.3529 E-02$

$3.9156 E-0$ ?

$2.8979 E-02-9.4365 E-03$

$3.0070 E-02-9.807 .5 E-03$

$3.0382 E-02-9.9081 E-03$

4.0301E-02 - $1.3146 E-0$ ?

$5.9049 E-02-1.9246 E-02$

3. $8665 E-02$

$3.5966 E-02$

$3.2825 E-02$

I. $7671 \mathrm{E}-02$
5.8271E-02 -1.9018E-0?

$5.422 .0 E-02-1.7684 E-02$

$4.8152 E-02-1.5696 E-02$ 
ATOM

CODE MASS FRACTION

ATOM

CODE

MASS FRACTION

CU $8.360000 E+00 \quad 1.000000 E+00$

FORMULA

$F$

$8 \cdot 3700000 E+00$
DENS ITY

RHO (G/CC)

$8.9399976 E \quad 00$
FUEL DENSITY

RHOF (G/CC)
TEMPERATURE

$T$ (DEG K)

0 .

PROBABILITY/PATH (/CM)

$\begin{array}{cl}\text { COLLIDE } & \text { MULT } \\ \text { SG } & \text { NUN } \\ 3.1281 E-01 & 0 . \\ 3.1875 E-01 & 0 . \\ 2.7637 E-01 & 0 . \\ 2.6578 E-01 & 0 . \\ 2.9546 E-01 & 0 . \\ 3.4210 E-01 & 0 . \\ 3.9425 E-01 & 0 . \\ 4.4555 E-01 & 0 . \\ 5.6519 E-01 & 0 . \\ 8.2283 E-01 & 0 . \\ 8.1112 E-01 & 0 . \\ 6.2102 E-01 & 0 . \\ 6.2847 E-01 & 0 . \\ 6.6398 E-01 & 0 . \\ 6.9413 E-01 & 0 . \\ 7.2018 E-01 & 0 . \\ 7.6531 E-01 & 0 . \\ 8.3005 E-01 & 0 . \\ 8.6962 E-01 & 0 .\end{array}$

SCATTER

$\operatorname{SGSO}(N, N)$

SGSI $(N, N)$

SGSO $(N, N+1) \quad S G S 1(N, N+1)$

2.9311E-01

$2.9867 E-01$

$9.7456 E-03$

$9.9331 E-03$

2.5895E-01

$2.4902 E-01$

$2.7682 E-01$

$8.6137 E-03$

$8.2772 E-03$

$9.2009 E-03$

$1.9684 \mathrm{E}-02$

$-6.4300 E-03$

$2.0058 E-02-6.5547 E-03$

$1.7391 E-02-5.6845 E-03$

$1.6724 E-02-5.4603 E-03$

$1.8591 E-02-6.0695 E-03$

3.2051E-01 1.0664E-02

$3.6936 E-01 \quad 1.2285 E-02$

4. $1742 E-01 \quad 1.3872 E-02$

$5.5608 E-01 \quad 8.8931 E-03$

8.0930E-01 I.2942E-02

$2.1525 E-02-7.0383 E-03$

$2.4806 E-02-8.1072 E-03$

$2.8033 E-02-9.1499 E-03$

$8.8887 E-03-2.9043 E-03$

$1.2936 E-02-4.2266 E-03$

$7.9679 E-01 \quad 1.2740 E-02$

$6.0698 E-01 \quad 9.7065 E-03$

$6.0698 E-01 \quad 9.7065 E-03$

$6.2574 E-01 \quad 1.1153 E-02$

$6.2642 E-01$

$1.3501 E-02$

$1.2736 E-02-4.1593 E-03$

$9.7022 E-03-3.1697 E-03$

$9.7022 E-03-3.1697 E-03$

$1.3407 E-02-4.3785 E-03$

$2.035 I E-02-6.6453 E-03$

$6.2191 E-01$

$1.3407 E-02$

$6.1657 E-01$

$5.9636 \mathrm{E}-01$

$1.3291 E-02$

$1.2506 E-02$

$6.2770 \mathrm{E}-03$
2.0205E-02 -6.6003E-03

$2.0031 E-02-6.5429 E-03$

$1.8333 E-02-5.9906 E-03$ 


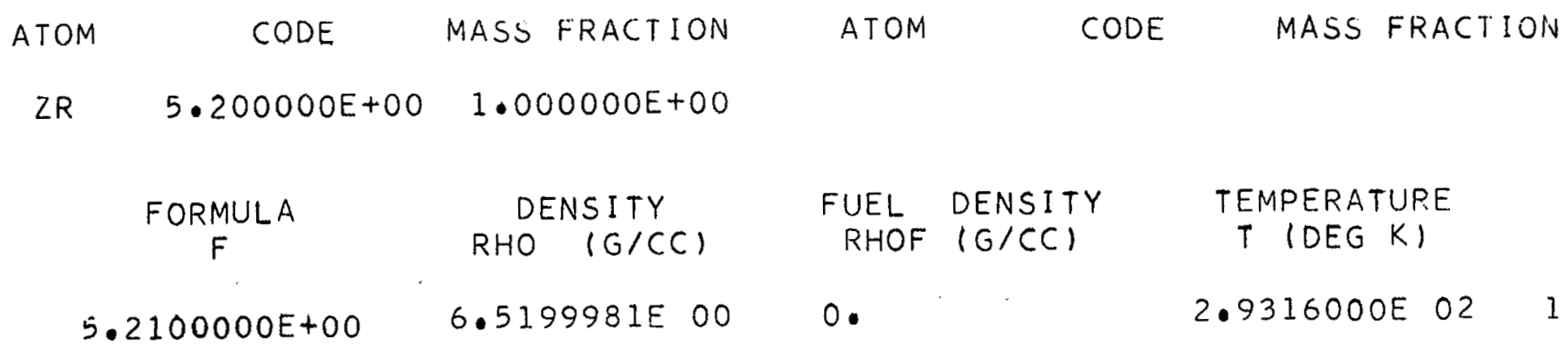

PROBABILITY/PATH (/CM)

\begin{tabular}{|c|c|c|c|c|c|}
\hline $\begin{array}{l}\text { COLLIDE } \\
\text { SG(N) }\end{array}$ & $\begin{array}{l}\text { MULTIPLY } \\
\text { NUSGF }(N)\end{array}$ & $S G S O(N, N)$ & $\begin{array}{r}S C \\
S G S I(N, N)\end{array}$ & $\begin{array}{l}\text { TER } \\
\text { SGSO }(N, N+1)\end{array}$ & $S G S I(N, N+1)$ \\
\hline $\begin{array}{l}1.7548 E-01 \\
1.7225 E-01 \\
1.8086 E-01 \\
2.1746 E-01 \\
2.7990 E-01\end{array}$ & $\begin{array}{l}0 . \\
0 . \\
0 . \\
0 . \\
0 .\end{array}$ & $\begin{array}{l}1.6774 E-01 \\
1.6466 E-01 \\
1.7289 E-01 \\
2.0788 E-01 \\
2.6757 E-01\end{array}$ & $\begin{array}{l}3.8615 E-03 \\
3.7909 E-03 \\
3.9806 E-03 \\
4.7833 E-03 \\
6.1481 E-03\end{array}$ & $\begin{array}{l}7.7343 \mathrm{E}-03 \\
7.5919 \mathrm{E}-03 \\
7.9715 \mathrm{E}-03 \\
9.5847 \mathrm{E}-03 \\
1.2337 \mathrm{E}-02\end{array}$ & $\begin{array}{l}-2.5630 E-03 \\
-2.5163 E-03 \\
-2.6423 E-03 \\
-3.1741 E-03 \\
-4.0768 E-03\end{array}$ \\
\hline $\begin{array}{l}3.4234 E-01 \\
3.7034 E-01 \\
3.7249 E-01 \\
3.6603 E-01 \\
3.1867 E-01\end{array}$ & $\begin{array}{l}0 . \\
0 . \\
0 . \\
0 . \\
0 .\end{array}$ & $\begin{array}{l}3.2725 E-01 \\
3.5401 E-01 \\
3.5607 E-01 \\
3.6199 E-01 \\
3.1515 E-01\end{array}$ & $\begin{array}{l}7.5252 E-03 \\
8.1391 E-03 \\
8.2039 E-03 \\
4.0407 E-03 \\
3.5221 E-03\end{array}$ & $\begin{array}{l}1.5089 E-02 \\
1.6322 E-02 \\
1.6418 E-02 \\
4.0331 E-03 \\
3.5113 E-03\end{array}$ & $\begin{array}{l}-4.9918 E-03 \\
-5.3986 E-03 \\
-5.4475 E-03 \\
-1.3321 E-03 \\
-1.1641 E-03\end{array}$ \\
\hline $\begin{array}{l}2.8425 E-01 \\
2.7570 E-01 \\
2.6706 E-01 \\
2.6695 E-01 \\
2.6708 E-01\end{array}$ & $\begin{array}{l}0 . \\
0 . \\
0 . \\
0 . \\
0 .\end{array}$ & $\begin{array}{l}2.8108 E-01 \\
2.7256 E-01 \\
2.6383 E-01 \\
2.6243 E-0 I \\
2.6005 E-01\end{array}$ & $\begin{array}{l}3.1423 E-03 \\
3.0444 E-03 \\
2.9475 E-03 \\
3.2665 E-03 \\
3.9085 E-03\end{array}$ & $\begin{array}{l}3.1317 E-03 \\
3.0367 E-03 \\
2.9395 E-03 \\
3.9130 E-03 \\
5.8600 E-03\end{array}$ & $\begin{array}{l}-1.0391 E-03 \\
-1.0050 E-03 \\
-9.7343 E-04 \\
-1.2956 E-03 \\
-1.9408 E-03\end{array}$ \\
\hline $\begin{array}{l}2.6698 E-01 \\
2.6694 E-0 I \\
2.6739 E-01 \\
2.6633 E-01\end{array}$ & $\begin{array}{l}0 . \\
0 . \\
0 .\end{array}$ & $\begin{array}{l}2.5921 E-01 \\
2.5794 E-01 \\
2.5658 E-01 \\
2.5946 E-01\end{array}$ & $\begin{array}{l}3.8964 E-03 \\
3.8781 E-03 \\
3.7519 E-03 \\
1.9200 E-03\end{array}$ & $\begin{array}{l}5.8410 E-03 \\
5.8126 E-03 \\
5.4737 E-03\end{array}$ & $\begin{array}{l}-1.9350 E-03 \\
-1.9263 E-03 \\
-1.8127 E-03\end{array}$ \\
\hline
\end{tabular}




$\begin{array}{ccc}\text { FORMULA } & \text { MASS } & \text { FUEL MASS } \\ \text { F } & \text { A }(\text { OXY } 16=16) & \text { AF }(\text { OXY16=16) } \\ 5.3600000 E \text { OO } & 9.2909999 E 01 & 0 .\end{array}$

\author{
TEMPERATURE \\ $T$ (DEG K)
}

$5.3600000 E 00$

$9.2909999 E$ OI

$2.9316000 E^{02}$

0

CROSS SECTION (BARN)

\begin{tabular}{|c|c|}
\hline $\begin{array}{l}\text { COLLID } \\
\text { SG(N) }\end{array}$ & \\
\hline $\begin{array}{l}2.3115 E \\
3.0148 E \\
3.9690 E \\
5.2243 E \\
6.7313 E\end{array}$ & $\begin{array}{l}00 \\
00 \\
00 \\
00 \\
00\end{array}$ \\
\hline $\begin{array}{l}8.0401 E \\
8.8015 E \\
9.0662 E \\
9.1262 E \\
8.2925 E\end{array}$ & $\begin{array}{ll}0 & 0 \\
0 & 0 \\
00 \\
00 \\
00\end{array}$ \\
\hline $\begin{array}{l}7.3860 E \\
7.3218 E \\
7.0637 E \\
6.4000 E \\
6.4000 E\end{array}$ & $\begin{array}{l}00 \\
00 \\
00 \\
00 \\
00\end{array}$ \\
\hline $\begin{array}{l}6.4000 E \\
6.4000 E \\
6.3999 E \\
6.4000 E\end{array}$ & $\begin{array}{l}00 \\
00 \\
00 \\
00\end{array}$ \\
\hline
\end{tabular}

\begin{tabular}{|c|c|c|c|c|}
\hline \multirow{2}{*}{\multicolumn{2}{|c|}{$\operatorname{SGSO}(N, N)$}} & \multicolumn{2}{|c|}{ SCATTER } & \multirow{3}{*}{$\begin{array}{l}\text { SGS } 1(N \cdot N+1) \\
-3.2615 E-02 \\
-4.2435 E-02 \\
-5.6040 E-02 \\
-7.3820 E-02 \\
-9.5196 E-02\end{array}$} \\
\hline & & $S G S 1(N, N)$ & SGSO & \\
\hline 2 & $\begin{array}{l}00 \\
00 \\
00\end{array}$ & $\begin{array}{l}4.9175 E-02 \\
6.4035 E-02 \\
8.4480 E-02 \\
1.1126 E-01 \\
1.4344 E-01\end{array}$ & $\begin{array}{l}E-02 \\
E-01 \\
E-01 \\
E-01 \\
E-01\end{array}$ & \\
\hline & $\begin{array}{l}00 \\
00 \\
00\end{array}$ & 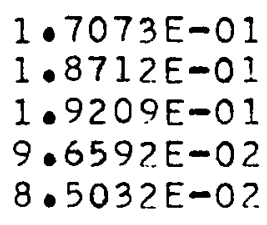 & $\begin{array}{l}3 \cdot 4 \\
3 \cdot 7 \\
3.8 \\
9.6 \\
8.4\end{array}$ & $\begin{array}{l}-1 \\
-1 \\
-1 \\
-3 \\
-7\end{array}$ \\
\hline 3 & $\begin{array}{l}00 \\
00 \\
00 \\
00\end{array}$ & $\begin{array}{l}7 E-02 \\
2 E-02 \\
0 E-0 ? \\
6 E-02 \\
9 E-0 ?\end{array}$ & $\begin{array}{l}-0 ? \\
-0 ? \\
-0 ? \\
-02 \\
-0 ?\end{array}$ & $\begin{array}{l}-2 \\
-2 \\
-? \\
-? \\
-4\end{array}$ \\
\hline $245 E$ & O & $\begin{array}{l}8.7409 E-02 \\
8.4581 E-02 \\
7.8399 E-02 \\
3.8900 E-02\end{array}$ & $\begin{array}{l}7 E-01 \\
4 E-01 \\
3 E-01\end{array}$ & $\begin{array}{l}-4 \cdot 3 \\
-4 \cdot 1 \\
-3.7\end{array}$ \\
\hline
\end{tabular}


*NIOBIUM

\begin{tabular}{|c|c|c|c|c|c|}
\hline ATOM & FORMUTA & MASS FRACTION & ATOM & FORMULA & MASS FRACTION \\
\hline NB & $5.3600000 \mathrm{E}+00$ & $1.0000000 \mathrm{E}+00$ & & & \\
\hline & $\begin{array}{c}\text { FORMULA } \\
\text { F }\end{array}$ & $\begin{array}{c}\text { DENS ITY } \\
\text { RHO }(G / C C)\end{array}$ & $\begin{array}{l}\text { FUEL } \\
\text { RHOF }\end{array}$ & $\begin{array}{l}\text { DENSITY } \\
(G / C C)\end{array}$ & $\begin{array}{l}\text { TEMPERATURE } \\
T(D E G \quad K)\end{array}$ \\
\hline & & $8.5699978 \mathrm{E}$ 00 & U. & & $2.9316000 E \quad 02$ \\
\hline
\end{tabular}

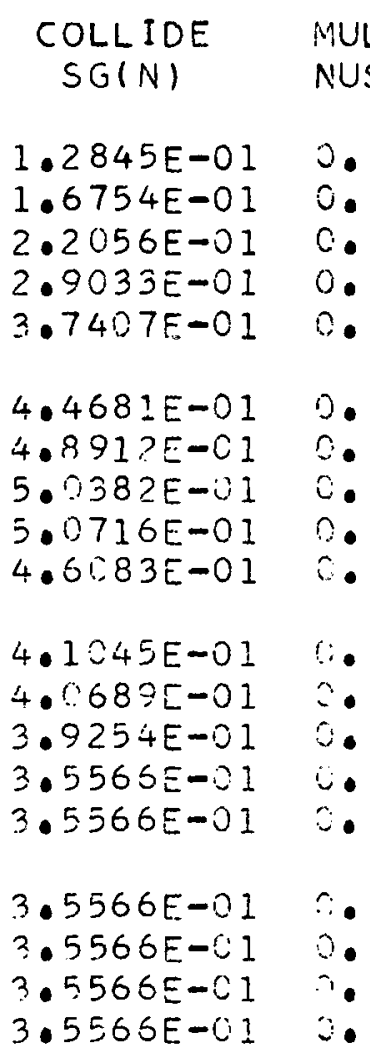

SCATTER

SGSO (N,N) SGSI(N,N) SGSO(N,N+I) SGSI(N,N+I)

$1.2233 E-01 \quad 2.7327 E-03$

$1.5956 E-01 \quad 3.5586 E-03$

$2.1009 E-01 \quad 4.6947 E-03$

2.7658E-01 6.1329E-03

$3.5636 E-01$ 7.971OE-03

$4 \cdot 2551 E-01$

$4.6540 E-01$

4. $7869 E-01$

$4 \cdot 94675-01$

$4 \cdot 3671 \equiv-11$

$9.4979 E-03$

$1.0399 E-02$

$1.0675 \mathrm{E}-02$

$5.3678 E-03$

$4.7254 E-03$

$3.53875-01$

$3.3624 E-i 1$

$3.5853 E-01$

$3.4564 E-C$ ?

$3.3900 E-01$

$3.3202 E-03$

$3.6378 E-03$

$3.8772 E-03$

4. $1671 E-03$

$4.9242 E-03$

3. $3312 E-\because 1$

$3.234 .3 E-21$

$3.2717 \mathrm{E}-\mathrm{O} 1$

$4 \cdot 2575 E-03$

$4.7004 E-03$

$4.2508 \mathrm{E}-03$

3.0145E-C1
$5.4823 E-03-1.8125 E-03$

$7.1507 E-03-2.3582 E-03$

$9.4152 E-03-3.1142 E-03$

$1.2395 E-02-4.1023 E-03$

$1.5970 E-02-5.2902 E-03$

$1.9068 E-02-5.2870 E-03$

2.0857E-02 - $5.8978 E-03$

$2.14 .52 E-02-7.073 \varepsilon E-03$

$5.3508 E-03-1.7751 E-03$

4. $1136 E-05-1.5606 E-03$

$3.8197 E-03-1.2635 E-03$

$3.6293 E-03-1.2010 E-03$

$3.8693 E-C 3-1.2798 E-03$

4.9936E-03-1.6525E-03

T.39S?E-03 -2.4401E-03

$7.2671 E-03-2.4179 E-0 ?$

$7.0509 E-03-2.33 C 2 E-03$

$6.35 ? 9 E-0.3-? \cdot 1062 E-03$ 
ATOM CODE MASS FRACTION

ATOM

CODE

MASS FRACTION

MO

$$
5.300000 E+00 \quad 1.000000 E+00
$$

FORMULA

$F$

$5.3100000 E+00$

\author{
DENSITY \\ RHO (G/CC)
}

$1.0200004 \mathrm{E} 01$ $\begin{array}{ll}\text { FUEL } & \text { DENSITY } \\ \text { RHOF } & (G / C C)\end{array}$

0 .

\section{TEMPERATURE \\ $T$ (DEG K)}

$2.9316000 E \quad 02 \quad 1$

PROBABILITY/PATH (/CM)

$\begin{array}{cc}\text { COLLIDE } & M U 1 \\ \text { SGIN) } & \text { NU } \\ 1.1382 E-01 & 0 . \\ 1.8751 E-01 & 0 . \\ 2.5961 E-01 & 0 . \\ 3.3492 E-01 & 0 . \\ 4.0706 E-01 & 0 . \\ 4.7601 E-01 & 0 . \\ 5.4979 E-01 & 0 . \\ 5.7878 E-01 & 0 . \\ 5.3320 E-01 & 0 . \\ 4.8153 E-01 & 0 . \\ & \\ 6.2861 E-01 & 0 . \\ 1.1154 E-00 & 0 . \\ 1.1464 E-00 & 0 . \\ 6.3115 E-01 & 0 . \\ 4.1492 E-01 & 0 . \\ & \\ 4.2584 E-01 & 0 . \\ 4.5015 E-01 & 0 . \\ 4.8147 E-01 & 0 . \\ 4.7735 E-01 & 0 .\end{array}$

SCATTER

SGSO $(N, N) \quad$ SGSI(N,N) SGSO $(N, N+1)$ SGSI $(N, N+1)$

$1.0894 E-01$

$1.7952 E-01$

$2.4857 \mathrm{E}-01$

3. $2068 E-01$

$3.8973 E-01$

$4.5571 E-01$

$5.2629 E-01$

$5.5391 E-01$

$5.2604 E-01$

4.7219E-01

$5.8848 E-01$

$8.5 .188 E-01$

8.1346E-0I

$5.1469 E-01$

$3.8305 E-01$

$3.7824 \mathrm{E}-01$

$3.7664 E-01$

3.6400E-01

$3.3535 E-01$
2.3640E-03

$3.9091 E-03$

$5.4050 E-03$

$6.9811 E-03$

$8.4613 E-03$

$9.9181 \mathrm{E}-03$

$1.1419 E-02$

$1.2049 E-02$

$5.5573 E-03$

4. $9770 E-03$

$7.1605 E-03$

$9.5534 E-03$

$9.5506 E-03$

$7.4060 E-03$

$5.0887 \mathrm{E}-03$

5.0890E-03

$5.0968 E-03$

4. 952$] E-03$

$2.3474 E-03$
4.7415E-03-1.5682E-03

$7.8137 \mathrm{E}-03-2.5977 \mathrm{E}-03$

$1.0819 E-02-3.5893 E-03$

$1.3958 E-02-4.6387 E-03$

$1.6963 E-02-5.6145 E-03$

1.9835E-02 -6.5893E-03

2.2906E-02 -7.5742E-03

$2.4109 E-02-8.0033 E-03$

$5.5430 E-03-1.8362 E-03$

$4.9565 E-03-1.6498 E-03$

7. $1477 E-03-2.3570 E-03$

$9.5362 E-03-3.1487 E-03$

$9.5329 E-03-3.1460 E-03$

$8.8904 E-03-2.9228 E-03$

$7.6356 E-03-2.5268 E-03$

$7.6350 E-03-2.5271 E-03$

$7.6349 E-03-2.5349 E-03$

$7.2346 E-03-2.3902 E-03$ 
ATOM CODE MASS FRACTION ATOM CODE MASS FRACTION

CD $\quad 8 \cdot 300000 E+00 \quad 1.000000 E+00$

$\underset{F}{\text { FORMULA }}$

$8.310000 C E+00$

\author{
DENSITY \\ RHO (G/CC)
}

$8.6479977 E \quad 00$

\section{FUEL DENSITY \\ RHOF $(G / C C)$}

\section{TEMPERATURE \\ $T$ (DEG K)}

$2.9316000 E \quad 02 \quad 1$

PROBABILITY/PATH $(/ C M)$

\begin{abstract}
COLLIDE
$S G(N)$

$1.9699 E-010$.

$1.8773 \mathrm{E}-01 \quad 0$.

2.1786E-01 0 .

$2.6884 E-01 \quad 0$.

$3.0593 E-01 \quad 0$.

$3.3143 E-010$.

$3.4302 E-01 \quad 0$.

$3.3839 E-01 \quad 0$.

3.2685E-01 0 .

$3.1310 E-010$.

3.0655E-01 0.

$3.0766 E-010$.

2.5968E-01 0 .

2.5145E-01 C.

$1.0429 E 000$.

$4.3747 E$ OI 0.

$1.2621 \mathrm{E} 02 \quad 0$.

$1.4709 \mathrm{E} O 2 \quad 0$.

$1.3974 \mathrm{E} 02 \quad 0$.
\end{abstract}

MULTIPLY

NUSGF $(N)$
SCATTER

SGSO $(N, N) \quad$ SGSI(N,N) SGSO(N,N+1) SGSI $(N, N+1)$

$1.8994 E-01 \quad 3.5394 E-03$

$7.0496 E-03-2.3575 E-03$

$1.8100 E-01 \quad 3.3773 E-03$

$2.1005 E-01 \quad 3.9027 E-03$

2.5921E-01 4.8330E-03

2. $9496 E-01 \quad 5.4777 E-03$

$6.7180 E-03-2.2510 E-03$

$7.7959 E-03-2.5956 E-03$

$9.6207 E-03-3.2200 E-03$

$1.0947 E-02-3.6422 E-03$

3.1954E-01

$3.3071 E-01$

3. $2624 E-01$

$3.2384 \mathrm{E}-01$

$3.1006 E-01$

$5.9395 E-03$

$6.1678 E-03$

$6.0715 E-03$

$2.9341 E-03$

$2.8096 E-03$

$1.1860 E-02-3.9511 E-03$

$1.2275 E-02-4.1098 E-03$

$1.2109 E-02-4.0414 E-03$

$2.9235 E-03-9.7348 E-04$

$2.7991 E-03-9.3240 E-04$

3.0317E-01

$2.7460 E-03$

$2.7369 E-03-9.1056 E-04$

3.0317E-01

2.5264E-01

2.2211E-01

$2.5036 E-01$

$2.7460 E-03$

$2.2937 E-03$

$2.2431 E-03$

$3.0578 E-03$

$2.7369 E-03-9.1056 E-04$

$2.2808 E-03-7.6412 E-04$

$2.6816 E-03-8.9436 E-04$

4.5616E-03-1.5282E-03

$6.5322 E-01$

$1.0470 E$ OO

$7.9581 E-03$

$1.1901 E-02-3.9673 E-03$

$7.5455 E-01$

$1.2736 E-02$

$1.9075 E-02-6.3393 E-03$

$8.9316 \mathrm{E}-03$

$1.3018 E-02$

$-4 \cdot 3262 E-03$
$2.8655 E-03$ 


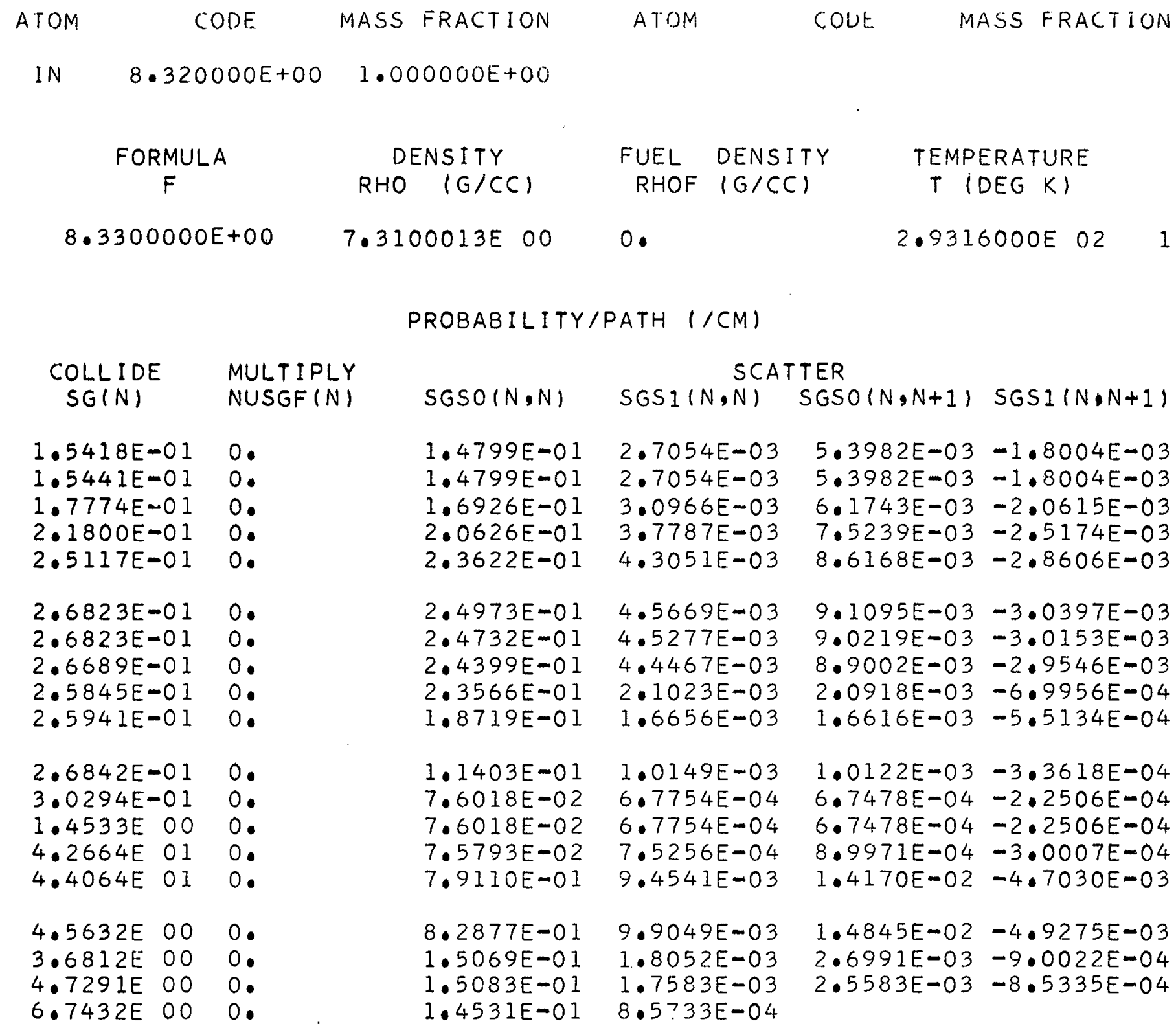




$$
5.420000 E+00 \quad 1.000000 E+00
$$

\section{FORMULA \\ $F$}

$5.4300000 E+00$

\author{
DENSITY \\ RHO (G/CC)
}
FUEL DENSITY RHOF $(G / C C)$

TEMPERATURE

$T$ (DEG K)

PROBABILITY/P.ATH (/CM)

\section{COLLIDE MULTIPLY $S G(N) \quad N U S G F(N)$}

$8.4433 E-02$

$8.4433 E-02$

8. $8272 E-02$

$1.0362 \mathrm{E}-01$

$1.0746 E-01$

$9.3646 E-02$

$8.3669 E-02$

$7.7530 E-02$

$9.9028 E-02$

$1.1517 \mathrm{E}-01$

$1.1523 E-01$

$1.3074 \mathrm{E}-01$

$1.4651 E-01$

$1.5110 E-01$

$1.4856 E-01$

$1.5416 E-01$

$1.6321 E-01$

$2.0237 E-01$

$2.4498 E-01$
$3.4999992 E \quad 00$

0.

\section{SCATTER}

$S G S O(N, N)$ SGSI $(N, N)$

SGSO $(N, N+I) \quad S G S I(N, N+I)$

8. $1962 E-02$

8. 1962E-02

$8.5687 E-02$

1.0059E-01

$1.0431 E-01$

1. $2468 E-03$

1. $2468 \mathrm{E}-03$

$1.3031 E-03$

$1.5287 E-03$

1. $5812 \mathrm{E}-03$

$9.0903 E-02$

$8.1217 E-02$

7. $5256 E-02$

$9.8291 E-02$

$1.1429 E-01$

$1.3824 E-03$

1. $2337 \mathrm{E}-03$

$1.1368 E-03$

$7.2984 E-04$

$8.44 .93 E-04$

1. 1429E-01

$1.2953 E-01$

8. 4493E-04

$9.5751 E-04$

$1.4477 E-01$

$1.4822 E-01$

1. $0720 E-03$

$1.2212 \mathrm{E}-03$

$1.4294 \mathrm{E}-03$

$1.4749 E-01$

$1.5354 E-01$

1. $8737 E-01$

$1.4650 E-03$

$1.5294 \mathrm{E}-03$

$1.8100 E-03$

$1.1224 \mathrm{E}-03$
$2.4704 E-03-8.3309 E-04$

$2.4704 E-03-8.3309 E-04$

$2.5827 E-03-8.7054 E-04$

$3.0318 E-03-1.0209 E-03$

$3.1441 E-03-1.0546 E-03$

$2.7399 E-03-9.2352 E-04$

$2.4479 E-03-8.2372 E-04$

$2.2682 E-03-7.5695 E-04$

$7.2427 E-04-2.4465 E-04$

$8.4214 E-04-2.8077 E-04$

$8.4214 E-04-2.8077 E-04$

$9.5442 E-04-3.1813 E-04$

$1.0667 E-03-3.5739 E-04$

$1.4597 \mathrm{E}-03-4.8774 \mathrm{E}-04$

$2.1335 E-03-7.1478 E-04$

2.1896E-03-7.3162E-04

$2.2794 E-03-7.6592 E-04$

$2.6345 E-03-8.7900 E-04$ 
ATOM

CODE

MASS FRACTION

$S M$

$$
8 \cdot 220000 E+00
$$

$1.000000 E+00$

$$
\begin{gathered}
\text { FORMULA } \\
\text { F }
\end{gathered}
$$

$8.2300000 E+00$

\author{
DENSI TY \\ RHO (G/CC)
}

$7.5400025 E \quad 00$
FUEL DENSITY RHOF (G/CC)

\begin{tabular}{|c|c|}
\hline $\begin{array}{l}\text { COLLIDE } \\
\text { SG(N) }\end{array}$ & \\
\hline $\begin{array}{l}3.0198 E-01 \\
3.0198 E-01 \\
3.0198 E-01 \\
3.0198 E-01 \\
3.0198 E-01\end{array}$ & \\
\hline $\begin{array}{l}3.0198 E-01 \\
3.0198 E-01 \\
3.0198 E-01 \\
3.7747 E-01 \\
5.2846 E-01\end{array}$ & \\
\hline 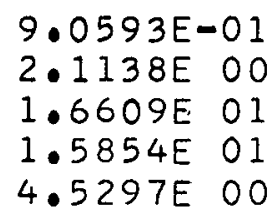 & \\
\hline $\begin{array}{ll}1.1324 E & 01 \\
1.7364 E & 02 \\
2.6526 E & 02 \\
2.3853 E & 02\end{array}$ & \\
\hline
\end{tabular}

PROBABILITYIPATH (/CM)

MULT IPLY

$\operatorname{NUSGF}(N)$

$\operatorname{SGSO}(N, N)$

SGSIIN,N

SCATTER

2.6447E-01

$2.6447 E-01$

$2.6447 E-01$

$2.6447 E-01$

$2.4978 E-01$

2.3509E-01

2. 2039E-0I

$1.9101 E-01$

$1.6497 \mathrm{E}-01$

$1.4997 \mathrm{E}-01$

$2.2496 E-O I$

4. $4992 \mathrm{E}-01$

$7.4987 E-01$

$5.9854 \mathrm{E}-0 \mathrm{I}$

$3.7240 E-01$

$4.4688 E-01$

$1.7130 E \quad 00$

$2.4956 \mathrm{E} \quad 00$

$1.6328 \mathrm{E} \quad 00$
$3.6899 E-03$

$3.6899 E-03$

$3.6899 E-03$

$3.6899 E-03$

$3.4,979 E-03$

$3.2943 E-03$

$3.0585 E-03$

$2.6559 \mathrm{E}-03$

$1.1252 E-03$

$1.0192 E-03$

$1.5290 E-03$

$3.0580 E-03$

$5.0967 E-03$

4. $5297 E-03$

$3.3981 E-03$

4.0777E-03

1.5631E-02

$2.2270 E-02$

$7 \cdot 3476 E-03$
SGSO $(N, N+1) \quad \operatorname{SGS} 1(N, N+1)$

$7.3057 E-03-2.4669 E-03$

$7.3057 E-03-2.4669 E-03$

$7.3057 E-03-2.4669 E-03$

$7.3057 E-03-2.4669 E-03$

$6.8999 E-03-2.3428 E-03$

$6.4941 E-03-2.2071 E-03$

$6.0879 E-03-2.0393 E-03$

$5.2762 E-03-1.7726 E-03$

$1.1162 E-03-3.7780 E-04$

$1.0146 E-03-3.3972 E-04$

$1.5220 E-03-5.0984 E-04$

$3.0440 E-03-1.0197 E-03$

$5.0733 E-03-1.6995 E-03$

$5.4115 E-03-1.8119 E-03$

$5.0733 E-03-1.6995 E-03$

$6.0879 E-03-2.0393 E-03$

$2.3337 E-02-7.8175 E-03$

$3.2202 E-02-J .0895 E-02$ 
ATOM

CODE MASS FRACTION

ATOM

CODE

MASS FRACTION

EU $8 \cdot 240000 E+00 \quad 1.000000 E+00$

$\begin{array}{ccclcl}\text { FORMULA } & \text { DENSITY } & \text { FUEL } & \text { DENSITY } & \text { TEMPERATURE } \\ \text { F } & \text { RHO (G/CC) } & \text { RHOF }(G / C C) & \text { T (DEG K) } & \\ 8.2500000 E+00 & 5.1659987 E 00 & 0 . & & 2.9316000 E 02 & 1\end{array}$

PROBABILITY/PATH (/CM)

\begin{tabular}{|c|c|c|c|c|c|}
\hline COLLIDE & MULT I PLY & & & TER & \\
\hline J) & $\operatorname{NUSGF}(N)$ & $\operatorname{SGSO}(N, N)$ & SGS & $\operatorname{SGSO}(N, N+1)$ & $\operatorname{SGS}_{1}(N, N+1)$ \\
\hline $\begin{array}{l}2.0476 E-01 \\
2.0476 E-01 \\
2.0476 E-01 \\
2.0476 E-01 \\
2.0476 E-01\end{array}$ & $\begin{array}{l}0 . \\
0 . \\
0 . \\
0 .\end{array}$ & $\begin{array}{l}1.7944 E-01 \\
1.7944 E-01 \\
1.7944 E-01 \\
1.7944 E-01 \\
1.6947 E-01\end{array}$ & $\begin{array}{l}2.4353 E-03 \\
2.4353 E-03 \\
2.4353 E-03 \\
2.4353 E-03 \\
2.3000 E-03\end{array}$ & $\begin{array}{l}4.8440 E-03 \\
4.8440 E-03 \\
4.8440 E-03 \\
4.8440 E-03 \\
4.5749 E-03\end{array}$ & $\begin{array}{l}-1.6245 E-03 \\
-1.6245 E-03 \\
-1.6245 E-03 \\
-1.6245 E-03 \\
-1.5342 E-03\end{array}$ \\
\hline $\begin{array}{l}2.0476 E-01 \\
2.0476 E-01 \\
2.0476 E-01 \\
2.5595 E-01 \\
3.5833 E-01\end{array}$ & $\begin{array}{l}0 . \\
0 . \\
0 . \\
0 .\end{array}$ & $\begin{array}{l}1.5950 E-01 \\
1.4953 E-01 \\
1.2960 E-01 \\
1.1188 E-01 \\
1.0171 E-01\end{array}$ & $\begin{array}{l}2.2225 E-03 \\
2.0295 E-03 \\
1.7589 E-03 \\
7.4371 E-04 \\
6.7610 E-04\end{array}$ & $\begin{array}{l}4.3064 E-03 \\
4.0367 E-03 \\
3.4985 E-03 \\
7.4006 E-04 \\
6.7278 E-04\end{array}$ & $\begin{array}{l}-1.5017 E-03 \\
-1.3537 E-03 \\
-1.1732 E-03 \\
-2.4819 E-04 \\
-2.2562 E-04\end{array}$ \\
\hline $\begin{array}{ll}1.2286 E & 00 \\
4.0952 E & 00 \\
6.1428 E & 00 \\
6.1428 E & 00 \\
2.8820 E & 01\end{array}$ & $\begin{array}{l}0 . \\
0 . \\
0 . \\
0 . \\
0 .\end{array}$ & $\begin{array}{l}2.0342 E-01 \\
3.5598 E-01 \\
4.0683 E-01 \\
3.0445 E-01 \\
2.5259 E-01\end{array}$ & $\begin{array}{l}1.3522 E-03 \\
2.3789 E-03 \\
2.7396 E-03 \\
2.2878 E-03 \\
2.2543 E-03\end{array}$ & $\begin{array}{l}1.3456 E-03 \\
2.3549 E-03 \\
2.6915 E-03 \\
2.6915 E-03 \\
3.3639 E-03\end{array}$ & $\begin{array}{l}-4.5125 E-04 \\
-8.0219 E-04 \\
-9.3768 E-04 \\
-9.3636 E-04 \\
-1.1281 E-03\end{array}$ \\
\hline 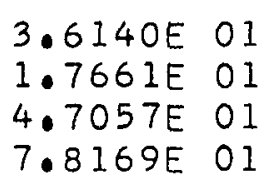 & $\begin{array}{l}0 . \\
0 \\
0 \\
0\end{array}$ & $\begin{array}{l}3.0311 E-01 \\
2.5259 E-01 \\
1.9397 E-01 \\
2.1623 E-01\end{array}$ & $\begin{array}{l}2.7052 E-03 \\
2.2543 E-03 \\
1.6866 E-03 \\
9.5140 E-04\end{array}$ & $\begin{array}{l}4.0367 E-03 \\
3.3639 E-03 \\
2.4468 E-03\end{array}$ & $\begin{array}{l}-1.3537 E-03 \\
-1.1281 E-03 \\
-8.2235 E-04\end{array}$ \\
\hline
\end{tabular}




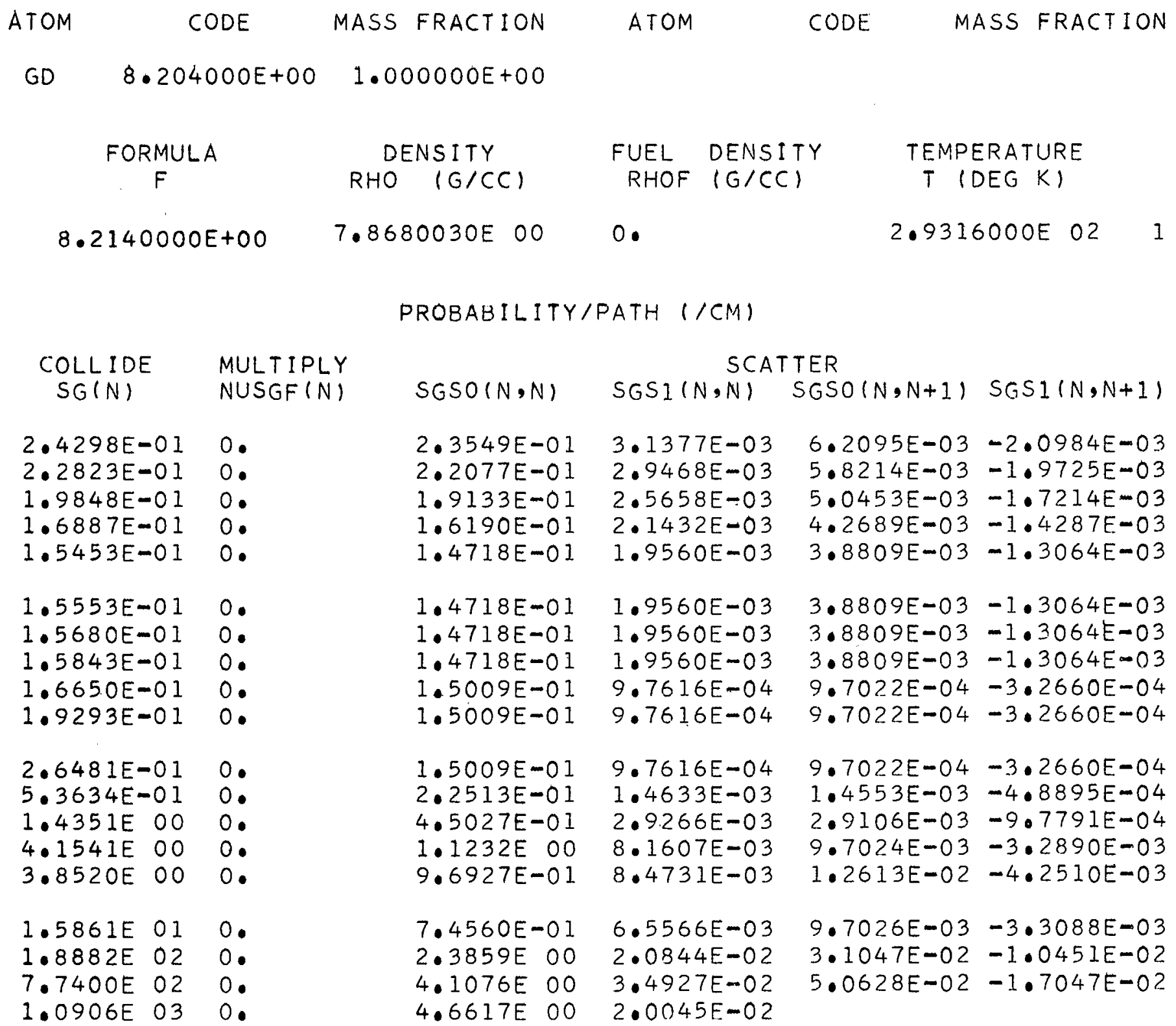




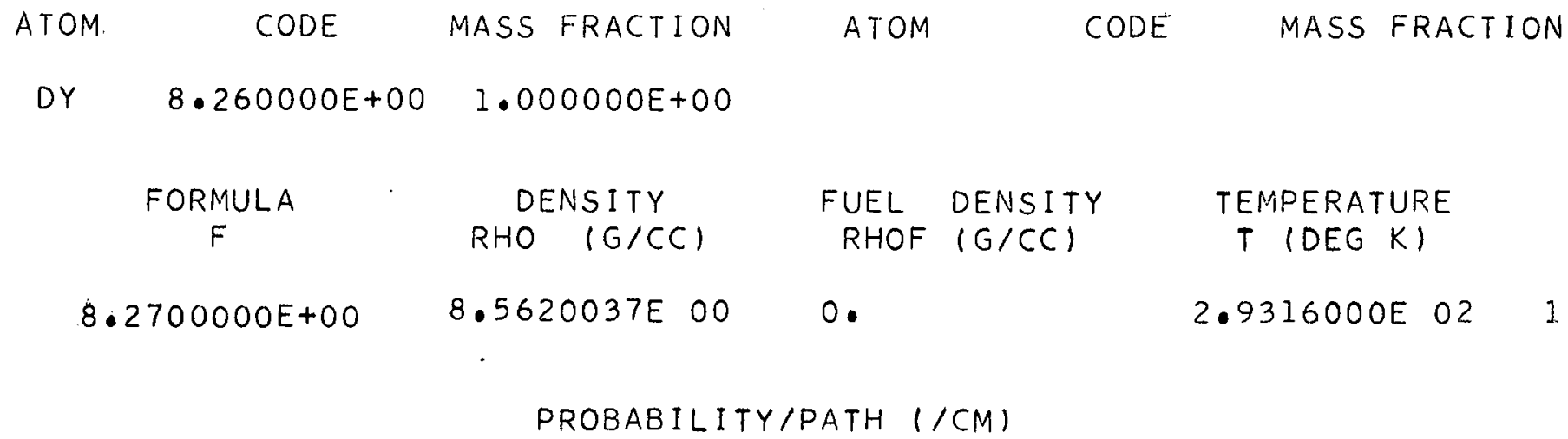

\section{COLLIDE MULTIPLY $S G(N) \quad$ NUSGF $(N)$}

PROBABILITY/PATH (/CM)

\begin{tabular}{|c|c|c|c|}
\hline \multicolumn{4}{|c|}{ SCATTER } \\
\hline$G S O(N, N)$ & $S G S I(N, N)$ & $S G S O(N, N+1)$ & $\operatorname{SGS1}(N, N+1)$ \\
\hline $\begin{array}{l}3.0964 E-01 \\
3.0964 E-01 \\
3.0964 E-01 \\
3.0964 E-01 \\
3.0964 E-01\end{array}$ & $\begin{array}{l}4.0066 E-03 \\
4.0066 E-03 \\
4.0066 E-03 \\
4.0066 E-03 \\
4.0066 E-03\end{array}$ & $\begin{array}{l}7.7778 E-03 \\
7.7778 E-03 \\
7.7778 E-03 \\
7.7778 E-03 \\
7.7778 E-03\end{array}$ & $\begin{array}{l}-2.7051 E-03 \\
-2.7051 E-03 \\
-2.7051 E-03 \\
-2.7051 E-03 \\
-2.7051 E-03\end{array}$ \\
\hline $\begin{array}{l}3.0964 E-01 \\
3.0964 E-01 \\
.0964 E-01 \\
3.1547 E-01 \\
.1547 E-01\end{array}$ & $\begin{array}{l}4.0066 E-03 \\
4.0066 E-03 \\
4.0066 E-03 \\
1.9777 E-03 \\
1.9777 E-03\end{array}$ & $\begin{array}{l}7.7778 E-03 \\
7.7778 E-03 \\
7.7778 E-03 \\
1.9445 E-03 \\
1.9445 E-03\end{array}$ & $\begin{array}{l}-2.7051 E-03 \\
-2.705 I E-03 \\
-2.7051 E-03 \\
-6.7629 E-04 \\
-6.7629 E-04\end{array}$ \\
\hline $\begin{array}{l}3.1547 E-01 \\
3.9434 E-01 \\
.9940 E-01 \\
.5558 E-01 \\
.3085 E-01\end{array}$ & $\begin{array}{l}1.9777 E-03 \\
2.4723 E-03 \\
3.7655 E-03 \\
5.3140 E-03 \\
7.0280 E-03\end{array}$ & $\begin{array}{l}1.9445 E-03 \\
2.4306 E-03 \\
3.6945 E-03 \\
6.2225 E-03 \\
1.0306 E-02\end{array}$ & $\begin{array}{l}-6.7629 E-04 \\
-8.4556 E-04 \\
-1.2928 E-03 \\
-2.1906 E-03 \\
-3.5793 E-03\end{array}$ \\
\hline 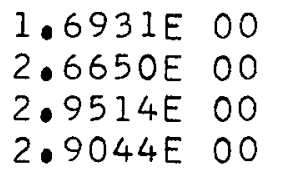 & $\begin{array}{l}1.4373 E-02 \\
2.2662 E-02 \\
2.4280 E-02 \\
1.1908 E-02\end{array}$ & $\begin{array}{l}2.1000 E-02 \\
3.3057 E-02 \\
3.4675 E-02\end{array}$ & $\begin{array}{l}-7.3451 E-03 \\
-1.1600 F-0 ? \\
-1.2037 E-02\end{array}$ \\
\hline
\end{tabular}




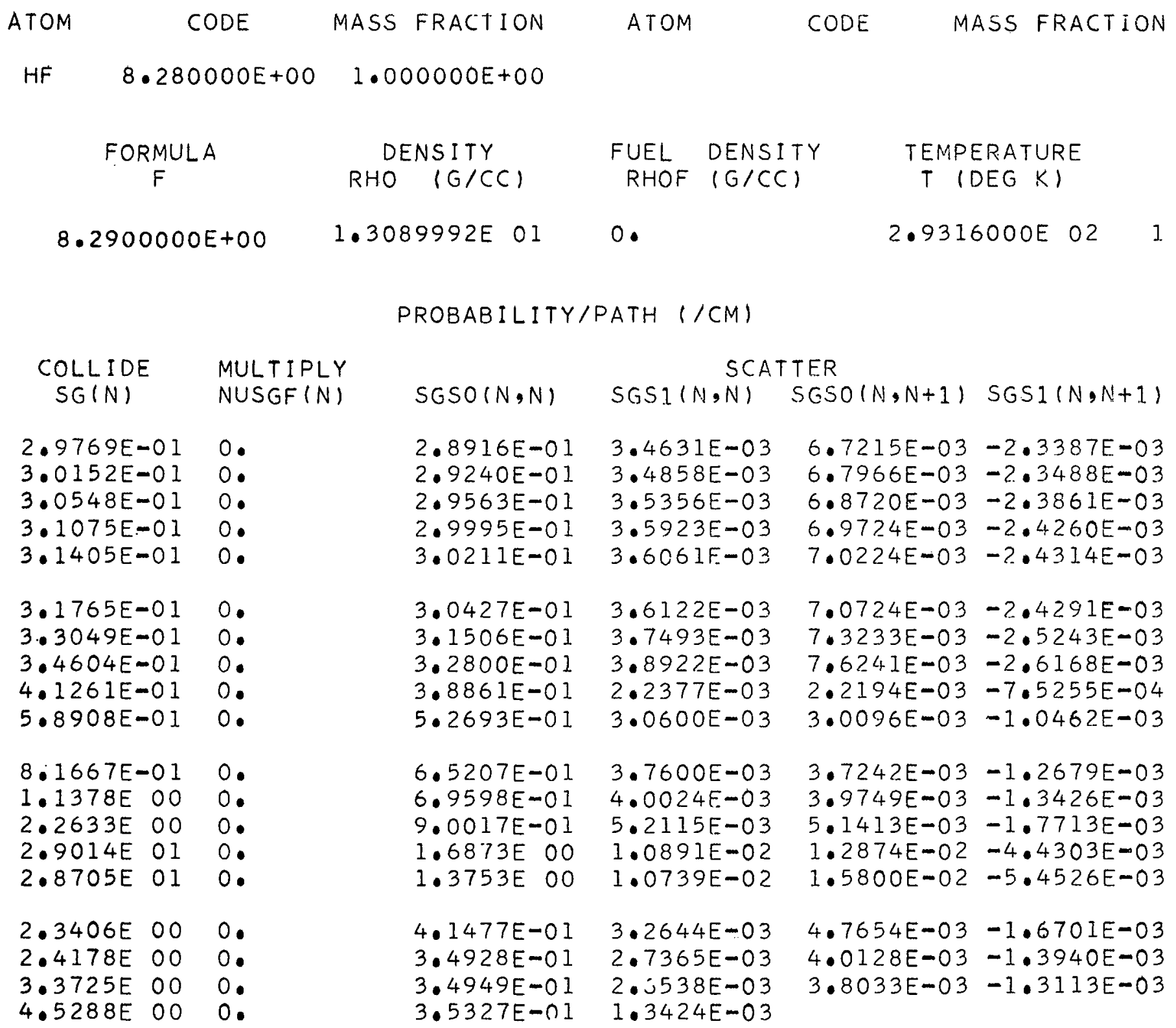


ATOM

CODE

MASS FRACTION

ATOM

CODE

MASS FRACTION

W

$8.370000 E+00 \quad 1.000000 E+00$

FORMULA

$F$

\author{
DENSITY \\ RHO (G/CC)
}
FUEL DENSITY RHOF (G/CC)

\section{TEMPERATURE \\ $T$ (DEG K)}

$1.9250004 E \quad 01$

0.

$2.9316000 E \quad 02 \quad 1$

PROBABILITY/PATH (/CM)

\section{COLLIDE MULTIPLY $S G(N)$ \\ NUSGF $(N)$}

$3.1851 E-01$

$3.6582 E-01$

4. $1629 E-01$

$4.3524 E-01$

$4 \cdot 3528 E-01$

4.4795E-01 0.

4.7639E-01 0 .

$5.1116 E-01$ 0.

$5.8095 E-010$.

$7.5891 \mathrm{E}-01$ 0.

$9.5190 E-01$

$1.1514 \mathrm{E} 00$

$2.0929 E 00$

$1.7041 \mathrm{E} \quad 00$

5. $2811 \mathrm{E}-01$

$6.2427 E-01$

8.2290E-01

$1.1822 \mathrm{E} \quad 00$

$1.3006 E 00$
0.
0.
0.
0.
0.
0.
0.
0.
0.
0.
0.
0.
0.
0.
0.
0.
SCATTER

SGSO N,N) SGSI(N,N) SGSO $(N, N+1)$ SGSI $(N, N+1)$

3. $1140 E-01$

$3.5765 E-01$

4. $0698 E-01$

4. $2547 E-01$

4.2547E-0I

4.3781E-01

4.6556E-01

4. $9947 E-01$

5.7692E-01

$7.5251 \mathrm{E}-01$

$9.4064 E-01$

$1.1288 \mathrm{E} \quad 00$

8. $1522 E-01$

3.6930E-0I

$3.3675 E-01$

3. $1180 E-01$

3. $1180 E-01$

3. $1198 \mathrm{E}-01$

$2.3792 E-O I$
$3.5571 E-03$
$4.1145 E-03$

4.6302E-03

$4 \cdot 9452 E-03$

4. $9452 E-03$

5.0505E-03

$5.3465 E-03$

$5.8181 E-03$

$3.2457 E-03$

4. 2072E-03

$5.2893 E-03$

$6.3533 E-03$

4.5670E-03

2.3086E-03

$2 \cdot 5352 E-03$

$2.3767 E-03$

$2.3767 E-03$

$2.3141 E-03$

$8.8029 E-04$
$7.0437 E-03-2.3789 E-03$

$8.0901 E-03-2.7613 E-03$

$9.2055 E-03-3.0903 E-03$

$9.6248 E-03-3.3353 E-03$

$9.6248 E-03-3.3353 E-03$

$9.9035 E-03-3.3939 E-03$

$1.0531 E-02-3.5850 E-03$

$1.1299 E-02-3.9283 E-03$

$3.2082 E-03-1.0992 E-03$

$4.1843 E-03-1.4074 E-03$

$5.2307 E-03-1.7896 E-03$

$6.2769 E-03-2.1537 E-03$

4.5331E-03-1.5339E-03

$2.7432 E-03-9.3204 E-04$

$3.7660 E-03-1.2753 E-03$

$3.4873 E-03-1.2101 E-03$

$3.4873 E-03-1.2101 E-03$

$3.3053 E-03-1.1475 E-03$ 


\begin{tabular}{|c|c|c|c|c|c|}
\hline \multicolumn{2}{|c|}{ CODE } & MASS & ATOM & \multicolumn{2}{|c|}{ MASS FRACTION } \\
\hline \multicolumn{2}{|c|}{$8 \cdot 340000 E+00$} & $1.000000 E+00$ & & & \\
\hline \multicolumn{2}{|c|}{$\begin{array}{c}\text { FORMULA } \\
\text { F }\end{array}$} & $\begin{array}{l}\text { DENSITY } \\
-10(G / C C)\end{array}$ & \multicolumn{2}{|c|}{$\begin{array}{l}\text { DENSITY } \\
(G / C C)\end{array}$} & $\begin{array}{c}\text { TEMPERATURE } \\
T \text { (DEG K) }\end{array}$ \\
\hline \multicolumn{2}{|c|}{$8.3500000 E+00$} & $1.9299994 \mathrm{E} \quad 01$ & 0 & \multicolumn{2}{|c|}{$2.9316000 E \quad 02$} \\
\hline \multicolumn{6}{|c|}{ PROBABILITY/PATH $(/ C M)$} \\
\hline $\begin{array}{l}\text { COLLIDE } \\
\text { SG }(N)\end{array}$ & $\begin{array}{l}\text { MULT IPLY } \\
\text { NUSGF }(N)\end{array}$ & $\operatorname{SGSO}(N, N)$ & $\operatorname{SGSI}(N, N))^{S C A T}$ & $\begin{array}{l}\text { TTER } \\
\text { SGSO }(N, N+1)\end{array}$ & $\operatorname{SGS} 1(N, N+1)$ \\
\hline $\begin{array}{l}3.0929 E-01 \\
3.8941 E-01 \\
4.1922 E-01 \\
3.6078 E-01 \\
3.1283 E-01\end{array}$ & $\begin{array}{l}0 . \\
0 . \\
0 . \\
0 . \\
0 .\end{array}$ & $\begin{array}{l}3.0126 E-01 \\
3.7903 E-01 \\
4.0708 E-01 \\
3.4839 E-01 \\
2.9924 E-01\end{array}$ & $\begin{array}{l}3.2398 E-03 \\
4.0749 E-03 \\
4.2895 E-03 \\
3.7655 E-03 \\
3.2038 E-03\end{array}$ & $\begin{array}{l}6.2530 E-03 \\
7.8672 E-03 \\
8.4487 E-03 \\
7.2313 E-03 \\
6.2109 E-03\end{array}$ & $\begin{array}{l}-2.1942 E-03 \\
-2.7594 E-03 \\
-2.8767 E-03 \\
-2.5564 E-03 \\
-2.1653 E-03\end{array}$ \\
\hline $\begin{array}{l}3.3437 E-01 \\
4.0225 E-01 \\
4.6186 E-01 \\
5.6220 E-01 \\
6.9058 E-01\end{array}$ & $\begin{array}{l}0 . \\
0 . \\
0 . \\
0 . \\
0 .\end{array}$ & $\begin{array}{l}3.1658 E-01 \\
3.7874 E-01 \\
4.3223 E-01 \\
5.1383 E-01 \\
5.6228 E-01\end{array}$ & $\begin{array}{l}3.4411 E-03 \\
4.1282 E-03 \\
4.6600 E-03 \\
2.6931 E-03 \\
2.9292 E-03\end{array}$ & $\begin{array}{l}6.5713 E-03 \\
7.8617 E-03 \\
8.9715 E-03 \\
2.6255 E-03 \\
2.8729 E-03\end{array}$ & $\begin{array}{l}-2.3423 E-03 \\
-2.8138 E-03 \\
-3.1599 E-03 \\
-9.3714 E-04 \\
-1.0077 E-03\end{array}$ \\
\hline 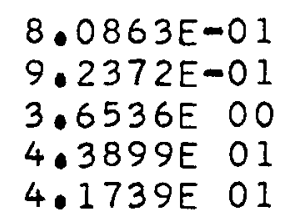 & $\begin{array}{l}0 . \\
0 . \\
0 . \\
0 .\end{array}$ & $\begin{array}{l}5.4613 E-01 \\
6.8413 E-01 \\
3.0536 E \quad 00 \\
3.5174 E \quad 00 \\
1.1480 E \quad 00\end{array}$ & $\begin{array}{l}2.8414 E-03 \\
3.5885 E-03 \\
1.5791 E-02 \\
2.0582 E-02 \\
8.0890 E-03\end{array}$ & $\begin{array}{l}2.7904 E-03 \\
3.4957 E-03 \\
1.5602 E-02 \\
2.4005 E-02 \\
1.1792 E-02\end{array}$ & $\begin{array}{l}-9.7509 E-04 \\
-1.2506 E-03 \\
-5.3553 E-03 \\
-8.5407 E-03 \\
-4.1456 E-03\end{array}$ \\
\hline $\begin{array}{ll}2.0245 E & 00 \\
2.7535 E & 00 \\
4.1219 E & 00 \\
5.5020 E & 00\end{array}$ & $\begin{array}{l}0 . \\
0 . \\
0 \\
0 .\end{array}$ & $\begin{array}{l}5.3458 E-01 \\
4.6739 E-01 \\
3.8538 E-01 \\
3.7624 E-01\end{array}$ & $\begin{array}{l}3.6949 E-03 \\
3.3261 E-03 \\
2.6223 E-03 \\
1.2792 E-03\end{array}$ & $\begin{array}{l}5.4904 E-03 \\
4.8010 E-03 \\
3.7496 E-03\end{array}$ & $\begin{array}{l}-1.8587 E-03 \\
-1.7207 E-03 \\
-1.2993 E-03\end{array}$ \\
\hline
\end{tabular}


HG $7.700000 E+00 \quad 1.000000 E+00$

\begin{tabular}{|c|c|c|c|c|}
\hline FORMULA & DENSITY & FUEL & DENSITY & TEMPERATURE \\
\hline$F$ & RHO $(G / C C)$ & RHOF & $(G / C C)$ & $T$ (DEG K) \\
\hline $.7100000 E+00$ & $1.3550000 E 01$ & 0. & & $2.9316000 E \quad 02$ \\
\hline
\end{tabular}

PROBABILITY/PATH (/CM)

\section{COLLIDE MULTIPLY \\ $S G(N) \quad N U S G F(N)$}

$2.1476 E-01$

$2.6894 E-01$

$2.8677 \mathrm{E}-01$

$2.5547 E-01$

$2.2347 E-01$

$2.5510 E-01$

$3.0932 E-01$

$3.5892 E-01$

$4 \cdot 2676 E-01$

$5: 1290 E-01$

$6.3318 E-01$

8. $2282 E-01$

$9.9699 E-01$

$1.0987 \mathrm{E} 00$

$1.7498 \mathrm{E} \quad 00$

$3.6726 \mathrm{E} \quad 00$

$6.9280 E \quad 00$

$1.1888 \mathrm{E} 01$

$1.4784 \mathrm{E} \quad 01$
0.
0 .
0.
0 .
0.
0.
0.
0.
0.

0.

0.

0.

0.

0 .

0.
0.
0.

\section{SCATTER}

SGSO $(N, N) \quad$ SGSI $(N, N) \quad$ SGSO $(N, N+1) \quad$ SGSI $(N, N+1)$

$2.0950 E-01$

$2.6232 E-01$

2.7946E-01

$2.4836 E-01$

2. $1647 E-01$

$2.2359 E-03$

2.8076E-03

$3.0195 E-03$

$2.6482 E-03$

$2.3375 E-03$

$2.4677 E-01$

$2.6613 E-03$

$3.2124 E-03$

$3.7338 E-03$

$2.1441 E-03$

$2.5117 E-03$

4. $8037 \mathrm{E}-01$

$5.4859 E-01$

5. $9718 E-01$

$6.6803 E-01$

$7.6793 \mathrm{E}-01$

$8.0559 E-01$

$2.8597 E-03$

$3.1085 E-03$

$3.4974 E-03$

4. $4917 E-03$

$5.6640 E-03$

$8.0559 E-01$

$8.0559 E-01$

8.0602E-01

$5.6640 E-03$

$5.6640 E-03$

$5.5141 E-03$

$2.7672 E-03$
$4.3481 E-03-1.5088 E-03$

$5.4446 E-03-1.8972 E-03$

$5.8006 E-03-2.0496 E-03$

$5.1549 E-03-1.7862 E-03$

$4.4932 E-03-1.5862 E-03$

$5.1220 E-03-1.8049 E-03$

$6.2059 E-03-2.1747 E-03$

7.1907E-03-2.5315E-03

2. $1130 E-03-7.3082 E-04$

$2.4545 E-03-8.7010 E-04$

$2.8030 E-03-9.8498 E-04$

$3.0512 E-03-1.0677 E-03$

$3.4134 E-03-1.2145 E-03$

$5.2407 E-03-1.8629 E-03$

$8.2745 E-03-2.8969 E-03$

$8.2745 E-03-2.8969 E-03$

$8.2745 E-03-2.8969 E-03$

$7.8427 E-03-2.7470 E-03$ 


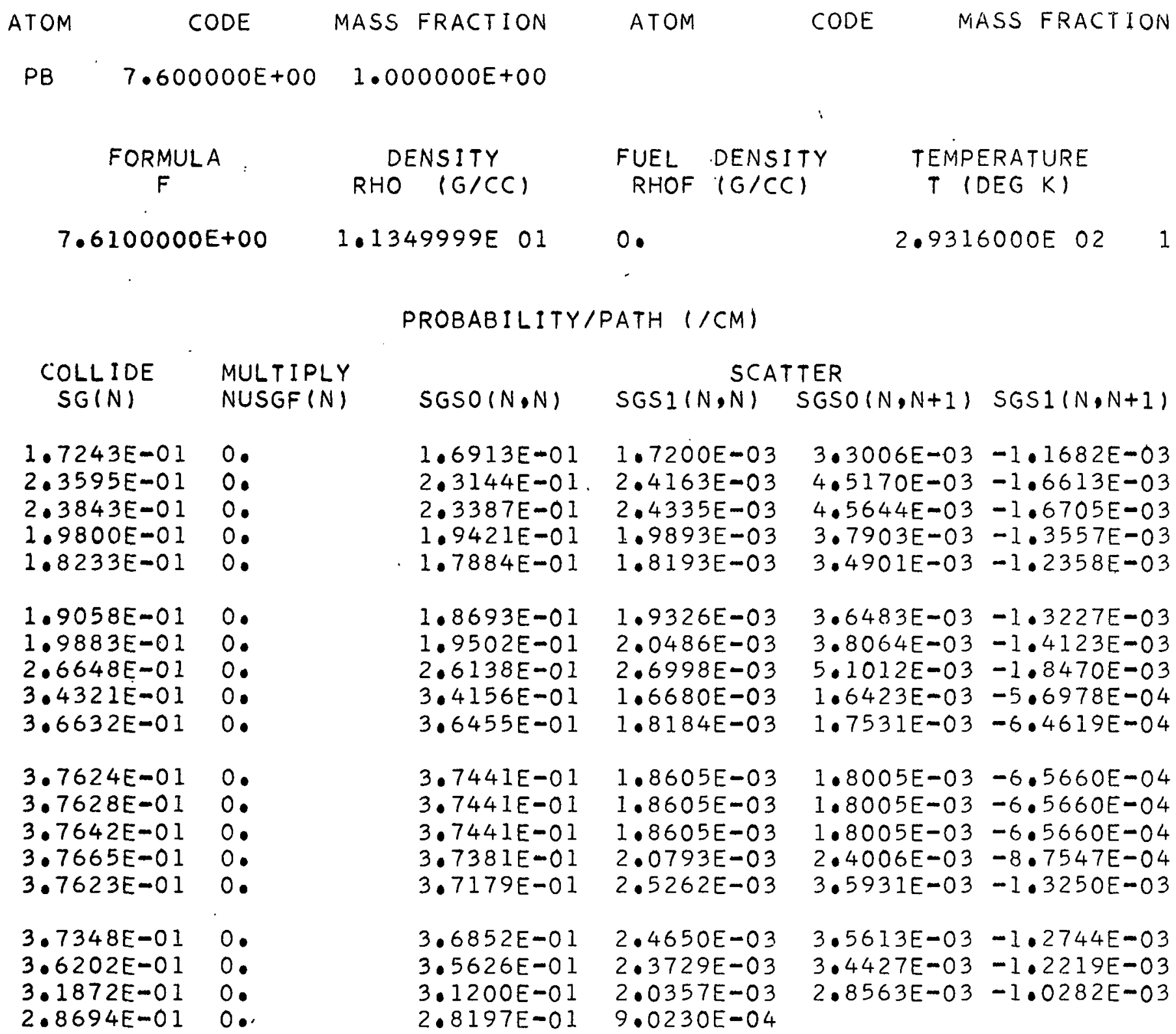


ATOM CODE MASS FRACTION ATOM CODE MASS FRACTION

BI $\quad 7 \cdot 500000 E+00 \quad 1 \cdot 000000 E+00$

$\begin{array}{ccccc}\text { FORMULA } & \text { DENSITY } & \text { FUEL } & \text { DENSITY } & \text { TEMPERATURE } \\ F & \text { RHO }(G / C C) & \text { RHOF }(G / C C) & T \text { (DEG K) }\end{array}$

$\begin{array}{lllllll}7.5100000 E+00 \quad 9.7470013 E & 00 & 0 & 0 & 2.9316000 E & 02 & 1\end{array}$

PROBABILITY/PATH (/CM)

\begin{tabular}{|c|c|c|c|c|c|}
\hline $\begin{array}{l}\text { COLLIDE } \\
\text { SG }(N)\end{array}$ & $\begin{array}{l}\text { MULTIPLY } \\
\text { NUSGF }(N)\end{array}$ & $\operatorname{SGSO}(N, N)$ & $\operatorname{SGSI}(N, N)$ & $\begin{array}{l}\text { TER } \\
\text { SGSO }(N, N+1)\end{array}$ & $\operatorname{SGSI}(N, N+1)$ \\
\hline $\begin{array}{l}1.5734 E-01 \\
2.0511 E-01 \\
2.0370 E-01 \\
1.5032 E-01 \\
1.2644 E-01\end{array}$ & $\begin{array}{l}0 . \\
0 . \\
0 . \\
0 .\end{array}$ & $\begin{array}{l}1.5433 E-01 \\
2.0118 E-01 \\
1.9980 E-01 \\
1.4744 E-01 \\
1.2402 E-01\end{array}$ & $\begin{array}{l}1.5611 E-03 \\
2.0908 E-03 \\
2.0746 E-03 \\
1.5168 E-03 \\
1.2814 E-03\end{array}$ & $\begin{array}{l}8 E-03 \\
5 E-03 \\
6 E-03 \\
5 E-03 \\
4 E-03\end{array}$ & $\begin{array}{l}-1.0576 E-03 \\
-1.4345 E-03 \\
-1.4227 E-03 \\
-1.0358 E-03 \\
-8.7676 E-04\end{array}$ \\
\hline $\begin{array}{l}1.5172 \mathrm{E}-01 \\
1.8825 \mathrm{E}-01 \\
2.1494 \mathrm{E}-01 \\
2.6552 \mathrm{E}-01 \\
3.1188 \mathrm{E}-01\end{array}$ & $\begin{array}{l}0 . \\
0 . \\
0 . \\
0 . \\
0 .\end{array}$ & $\begin{array}{l}1.4882 E-01 \\
1.8465 E-01 \\
2.1083 E-01 \\
2.6425 E-01 \\
3.1039 E-01\end{array}$ & $\begin{array}{l}1.5675 E-03 \\
1.8884 E-03 \\
2.1582 E-03 \\
1.2958 E-03 \\
1.5392 E-03\end{array}$ & $\begin{array}{l}2.9047 E-03 \\
3.6035 E-03 \\
4.1145 E-03 \\
1.2706 E-03 \\
1.4926 E-03\end{array}$ & $\begin{array}{l}-1.0820 E-03 \\
-1.2860 E-03 \\
-1.4703 E-03 \\
-4.4619 E-04 \\
-5.4116 E-04\end{array}$ \\
\hline $\begin{array}{l}2.8660 E-01 \\
2.5570 E-01 \\
2.5853 E-01 \\
2.5856 E-01 \\
2.5568 E-01\end{array}$ & $\begin{array}{l}0 . \\
0 . \\
0 . \\
0 .\end{array}$ & $\begin{array}{l}2.8522 E-01 \\
2.5446 E-01 \\
2.5726 E-01 \\
2.5684 E-01 \\
2.5310 E-01\end{array}$ & $\begin{array}{l}1.3942 E-03 \\
1.2640 E-03 \\
1.2721 E-03 \\
1.4204 E-03 \\
1.6834 E-03\end{array}$ & $\begin{array}{l}1.3714 E-03 \\
1.2237 E-03 \\
1.2371 E-03 \\
1.6494 E-03 \\
2.4458 E-03\end{array}$ & $\begin{array}{l}-4.7713 E-04 \\
-4.4585 E-04 \\
-4.4489 E-04 \\
-5.9319 E-04 \\
-8.6565 E-04\end{array}$ \\
\hline $\begin{array}{l}.4860 E-01 \\
.4158 E-01 \\
4483 E-01 \\
4897 E-01\end{array}$ & $\begin{array}{l}0 . \\
0 . \\
0 . \\
0 .\end{array}$ & $\begin{array}{l}2.4600 E-01 \\
2.3890 E-01 \\
2.4200 E-01 \\
2.4817 E-01\end{array}$ & $\begin{array}{l}1.6513 \mathrm{E}-03 \\
1.6184 \mathrm{E}-03 \\
1.5898 \mathrm{E}-03 \\
7.9415 \mathrm{E}-04\end{array}$ & $\begin{array}{l}2.3773 E-03 \\
2.3088 E-03 \\
2.2155 E-03\end{array}$ & $\begin{array}{l}-8.5652 E-04 \\
-8.4651 E-04 \\
-8.0833 E-04\end{array}$ \\
\hline
\end{tabular}




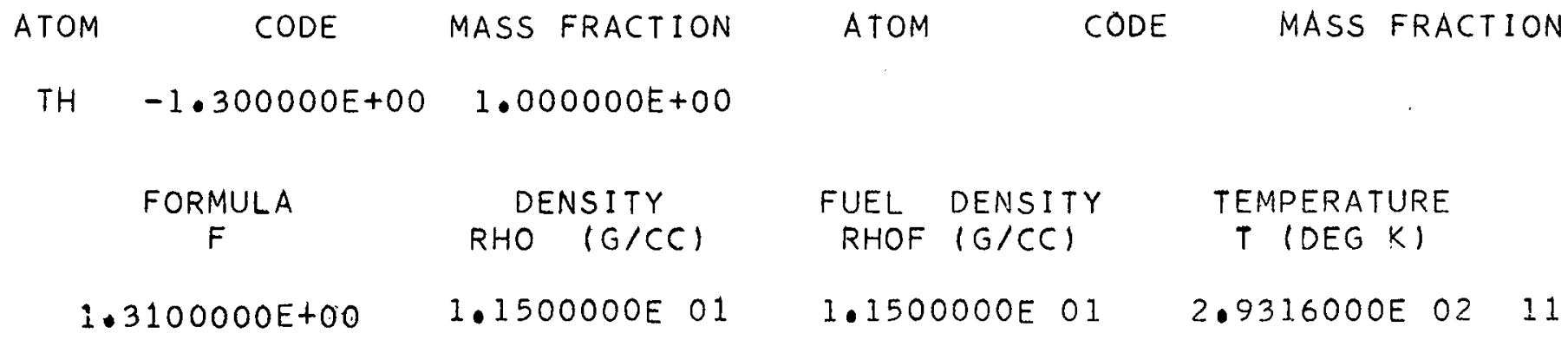

PROBABILITY/PATH (/CM)

$\begin{array}{cl}\text { COLLIDE } & \text { MULTIPLY } \\ \text { SGIN) } & \text { NUSGF(N) } \\ 2.4050 E-01 & 2.6573 E-02 \\ 2.3438 E-01 & 1.1346 E-02 \\ 2.2035 E-01 & 9.8530 E-03 \\ 1.9751 E-01 & 4.9265 E-03 \\ 1.9975 E-01 & 7.4644 E-04 \\ 2.2244 E-01 & 0 . \\ 2.6051 E-01 & 0 . \\ 3.0589 E-01 & 0 . \\ 3.7546 E-01 & 0 . \\ 4.4891 E-01 & 0 . \\ 4.4488 E-01 & 0 . \\ 1.3660 E-00 & 0 . \\ 1.3346 E-00 & 0 . \\ 3.6426 E-01 & 0 . \\ 3.9412 E-01 & 0 . \\ 4.2696 E-01 & 0 . \\ 4.5831 E-01 & 0 . \\ 5.2028 E-01 & 0 . \\ 5.5485 E-01 & 0 .\end{array}$

\section{PROBABILITY/PATH (}

$\operatorname{SGSO}(N, N) \quad \operatorname{SGSATTER}$

$2.2591 E-01 \quad 2.0548 E-03$

$2.2591 E-01 \quad 2.0548 E-03$

$2.1271 E-01 \quad 1.9942 E-03$

$1.9217 E-01 \quad 1.7651 E-03$

$1.9511 E-01 \quad 1.8224 E-03$

$2.1564 E-01$

2. 5085E-OI

2. $9339 E-01$

$3.6119 E-01$

$4.2660 E-01$

$1.9453 E-03$

$2.2969 E-03$

$2.7709 E-03$

$1.6263 E-03$

$1.8835 E-03$

4. 1916E-01

$3.8200 E-01$

3. 5822E-01

$3.4583 E-01$

3. $5814 E-01$

$1.8674 E-03$

$1.7290 E-03$

$1.5923 E-03$

$1.7462 E-03$

$2.1655 E-03$

$3.6702 E-01$

$3.6554 E-01$

3.7201E-OI

$3.7026 E-01$
$2 \cdot 2014 E-03$

$2.2562 E-03$

$2.1656 E-03$

$1.0738 E-03$
$3.9892 E-03-1.3881 E-03$

$3.9892 E-03-1.3881 E-03$

$3.7564 E-03-1.3665 E-03$

$3.3935 E-03-1.1980 E-03$

$3.4455 E-03-1.2466 E-03$

$3.8077 E-03-1.3089 E-03$

4.4296E-03-1.5565E-03

$5.1814 E-03-1.9051 E-03$

$1.5738 E-03-5.7430 E-04$

$1.8585 E-03-6.4098 E-04$

$1.8263 E-03-6.4650 E-04$

$1.6646 E-03-6.1635 E-04$

$1.5607 E-03-5.4894 E-04$

2.0121E-03-7.3751E-04

$3.1345 E-03-1.1178 E-03$

$3.2121 E-03-1.1277 E-03$

$3.1996 E-03-1.1868 E-03$

$3.0845 E-03-1.0778 E-03$ 


\begin{tabular}{|c|c|c|c|c|c|}
\hline ATOM & CODE & MASS FRACTION & ATOM & CODE & MASS FRACTION \\
\hline 233 & $-1.000000 E+00$ & $1.000000 E+00$ & & & \\
\hline - & $\begin{array}{c}\text { FORMULA } \\
\mathrm{F}\end{array}$ & $\begin{array}{c}\text { DENSITY } \\
\text { RHO }(G / C C)\end{array}$ & $\begin{array}{l}\text { FUEL } \\
\text { RHOF }\end{array}$ & $\begin{array}{l}\text { DENSITY } \\
(G / C C)\end{array}$ & $\begin{array}{l}\text { TEMPERATURE } \\
T(D E G \quad K)\end{array}$ \\
\hline & $0000 E+00$ & $1.8288481 \mathrm{E} O 1$ & 1.8288 & $3481 E$ OI & $2.9316000 E \quad 02$ \\
\hline
\end{tabular}

PROBABILITY/PATH (/CM)

COLLIDE MULTIPLY $S G(N) \quad N U S G F(N)$
SCATTER

SGSO $(N, N) \quad$ SGSI(N,N) SGSO $(N, N+1)$ SGSI $(N, N+1)$

\begin{tabular}{|c|c|c|c|c|c|}
\hline $\begin{array}{l}5.7192 E-01 \\
5.7192 E-01\end{array}$ & $\begin{array}{l}2.3349 E-0 I \\
2.3468 E-0 I\end{array}$ & $\begin{array}{l}4.6446 E-01 \\
4.6446 E-01\end{array}$ & $\begin{array}{l}4.3852 E-03 \\
4.3852 E-03\end{array}$ & $\begin{array}{l}8.2024 E-03 \\
8.2024 E-03\end{array}$ & $\begin{array}{l}-3.0145 E-03 \\
-3.0145 E-03\end{array}$ \\
\hline $\begin{array}{l}.7665 E-01 \\
.8846 E-01 \\
.9083 E-01 \\
.3100 E-01 \\
.0469 E \quad 00\end{array}$ & $\begin{array}{l}2.4484 E-01 \\
2.7060 E-01 \\
2.7320 E-01 \\
3.6442 E-01 \\
1.4163 E \quad 00\end{array}$ & $\begin{array}{l}4.6446 E-01 \\
4.6446 E-01 \\
4.6446 E-01 \\
4.7061 E-01 \\
4.7061 E-01\end{array}$ & $\begin{array}{l}4.3852 E-03 \\
4.3852 E-03 \\
4.3852 E-03 \\
2.1243 E-03 \\
2.1243 E-03\end{array}$ & $\begin{array}{l}8.2024 E-03 \\
8.2024 E-03 \\
8.2024 E-03 \\
2.0506 E-03 \\
2.0506 E-03\end{array}$ & $\begin{array}{l}145 E-03 \\
145 E-03 \\
145 E-03 \\
363 E-04 \\
363 E-04\end{array}$ \\
\hline $\begin{array}{ll}2.0797 E & 00 \\
2.7887 E & 00 \\
3.7813 E & 00 \\
1.0399 E & 01 \\
1.1344 E & 01\end{array}$ & $\begin{array}{ll}3.6631 E & 00 \\
5.3174 E & 00 \\
7.5626 E & 00 \\
2.2097 E & 01 \\
2.3964 E & 01\end{array}$ & $\begin{array}{l}4.7061 E-01 \\
4.7061 E-01 \\
4.7061 E-01 \\
4.6993 E-01 \\
4.6856 E-01\end{array}$ & $\begin{array}{l}2.1243 E-03 \\
2.1243 E-03 \\
2.1243 E-03 \\
2.3756 E-03 \\
2.8780 E-03\end{array}$ & $\begin{array}{l}2.0506 E-03 \\
2.0506 E-03 \\
2.0506 E-03 \\
2.7341 E-03 \\
4.1012 E-03\end{array}$ & $\begin{array}{l}-7.5363 E-04 \\
-7.5363 E-04 \\
-7.5363 E-04 \\
-1.0048 E-03 \\
-1.5073 E-03\end{array}$ \\
\hline 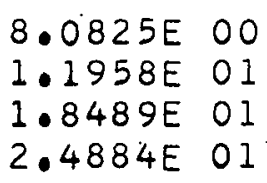 & $\begin{array}{ll}1.5905 E & 01 \\
2.5524 E & 01 \\
4.4245 E & 01 \\
5.6209 E & 01\end{array}$ & $\begin{array}{l}4.6855 E-01 \\
4.6856 E-01 \\
4.6877 E-01 \\
4.7266 E-01\end{array}$ & $\begin{array}{l}2.8780 E-03 \\
2.8780 E-03 \\
2.8000 E-03 \\
1.3707 E-03\end{array}$ & $\begin{array}{l}\text { 4. } 1012 E-03 \\
4.1012 E-03 \\
3.8872 E-03\end{array}$ & $\begin{array}{l}-1.5073 E-03 \\
-1.5073 E-03 \\
-1.4292 E-03\end{array}$ \\
\hline
\end{tabular}

$5.7192 E-01$

$5.7192 E-01$

$2.4484 E-01$

$2.7060 E-01$

$2.7320 E-01$

$3.6442 E-01$

E

4.7061E-0I

4.7061E-01

4.7061E-01

$4.6993 E-01$

.6856E-01

$2.8780 E-03$

$2.8780 E-03$

$1.3707 E-03$
$8.2024 E-03-3.0145 E-03$

$8.2024 E-03-3.0145 E-03$

$8.2024 E-03-3.0145 E-03$

$8.2024 E-03-3.0145 E-03$

$2.0506 E-03-7.5363 E-04$

$2.0506 E-03-7.5363 E-04$

$2.0506 E-03-7.5363 E-04$

$2.0506 E-03-7.5363 E-04$

$2.0506 E-03-7.5363 E-04$

$2.7341 E-03-1.0048 E-03$

4.1012E-03-1.5073E-03

$3.8872 E-03-1.4292 E-03$ 


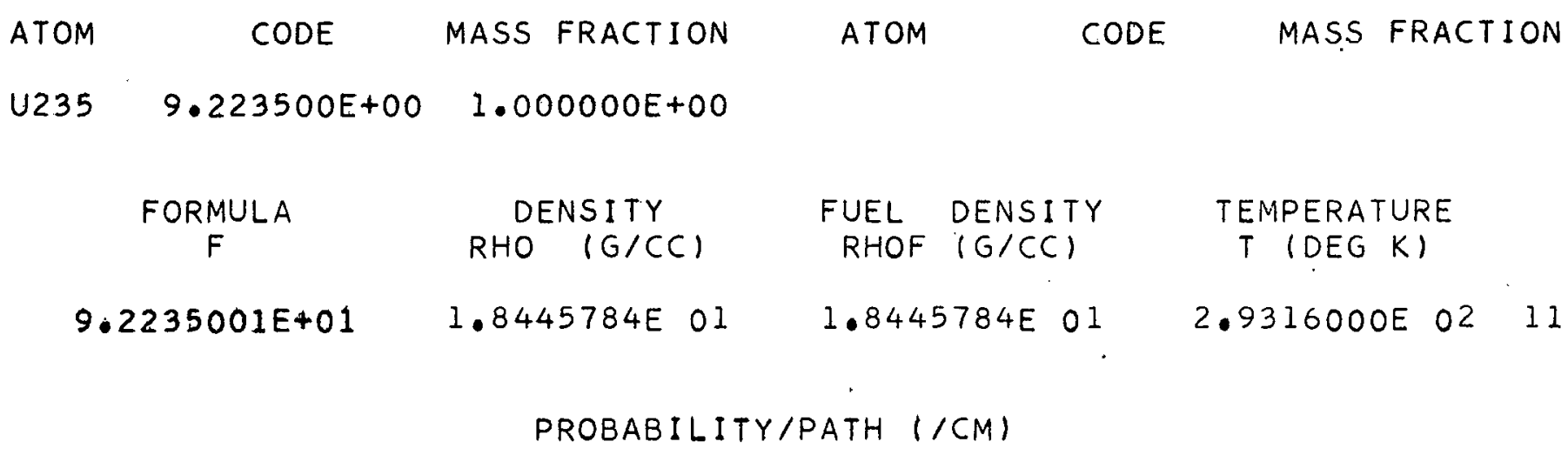

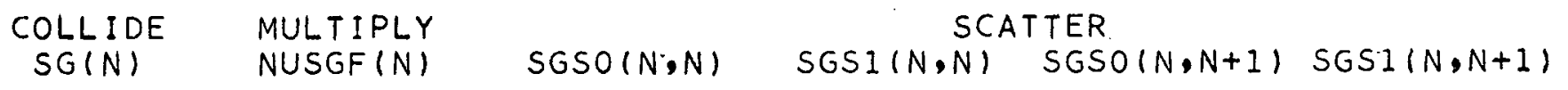

\begin{tabular}{|c|c|c|c|c|c|}
\hline $\begin{array}{l}2.2451 E-01 \\
2.4342 E-01\end{array}$ & $\begin{array}{l}1.9819 E-01 \\
1.7628 E-01\end{array}$ & $\begin{array}{l}1.5104 E-01 \\
1.6731 E-01\end{array}$ & $\begin{array}{l}1.3143 E-03 \\
1.4586 E-03\end{array}$ & $\begin{array}{l}2.5737 E-03 \\
2.8509 E-03\end{array}$ & $\begin{array}{l}-8.8419 E-04 \\
-9.8220 E-04\end{array}$ \\
\hline $\begin{array}{l}2.5524 E-01 \\
2.9541 E-01 \\
3.6395 E-0 I \\
4.7739 E-01 \\
6.1682 E-01\end{array}$ & $\begin{array}{l}1.6951 E-01 \\
1.7843 E-01 \\
1.8999 E-01 \\
2.6026 E-01 \\
4.2657 E-01\end{array}$ & $\begin{array}{l}1.7428 E-01 \\
2.0681 E-01 \\
2.6723 E-01 \\
3.5066 E-01 \\
4.0008 E-01\end{array}$ & $\begin{array}{l}1.5543 E-03 \\
1.8554 E-03 \\
2.3862 E-03 \\
1.4862 E-03 \\
1.7009 E-03\end{array}$ & $\begin{array}{l}2.9699 E-03 \\
3.5243 E-03 \\
4.5539 E-03 \\
1.4749 E-03 \\
1.6828 E-03\end{array}$ & $\begin{array}{l}-1.0580 E-03 \\
-1.2664 E-03 \\
-1.6253 E-03 \\
-5.0021 E-04 \\
-5.7593 E-04\end{array}$ \\
\hline 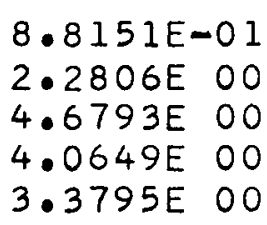 & 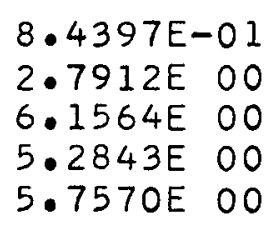 & $\begin{array}{l}4.0008 E-01 \\
4.0008 E-01 \\
4.0008 E-01 \\
3.9952 E-01 \\
3.9840 E-01\end{array}$ & $\begin{array}{l}1.7009 E-03 \\
1.7009 E-03 \\
1.7009 E-03 \\
1.8928 E-03 \\
2.2768 E-03\end{array}$ & $\begin{array}{l}1.6828 E-03 \\
1.6828 E-03 \\
1.6828 E-03 \\
2.2437 E-03 \\
3.3656 E-03\end{array}$ & $\begin{array}{l}-5.7593 E-04 \\
-5.7593 E-04 \\
-5.7593 E-04 \\
-7.6790 E-04 \\
-1.1519 E-03\end{array}$ \\
\hline 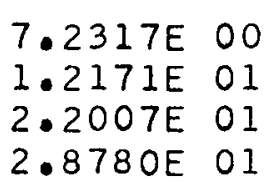 & $\begin{array}{ll}1.3896 E & 01 \\
2.4186 E & 01 \\
4.5082 E & 01 \\
5.8468 E & 01\end{array}$ & $\begin{array}{l}3.9840 E-01 \\
3.9840 E-01 \\
4.0812 E-01 \\
4.0176 E-01\end{array}$ & $\begin{array}{l}2.2768 E-03 \\
2.2768 E-03 \\
2.2442 E-03 \\
1.1249 E-03\end{array}$ & $\begin{array}{l}3.3656 E-03 \\
3.3656 E-03 \\
3.2662 E-03\end{array}$ & $\begin{array}{l}-1.1519 E-03 \\
-1.1519 E-03 \\
-1.0923 E-03\end{array}$ \\
\hline
\end{tabular}




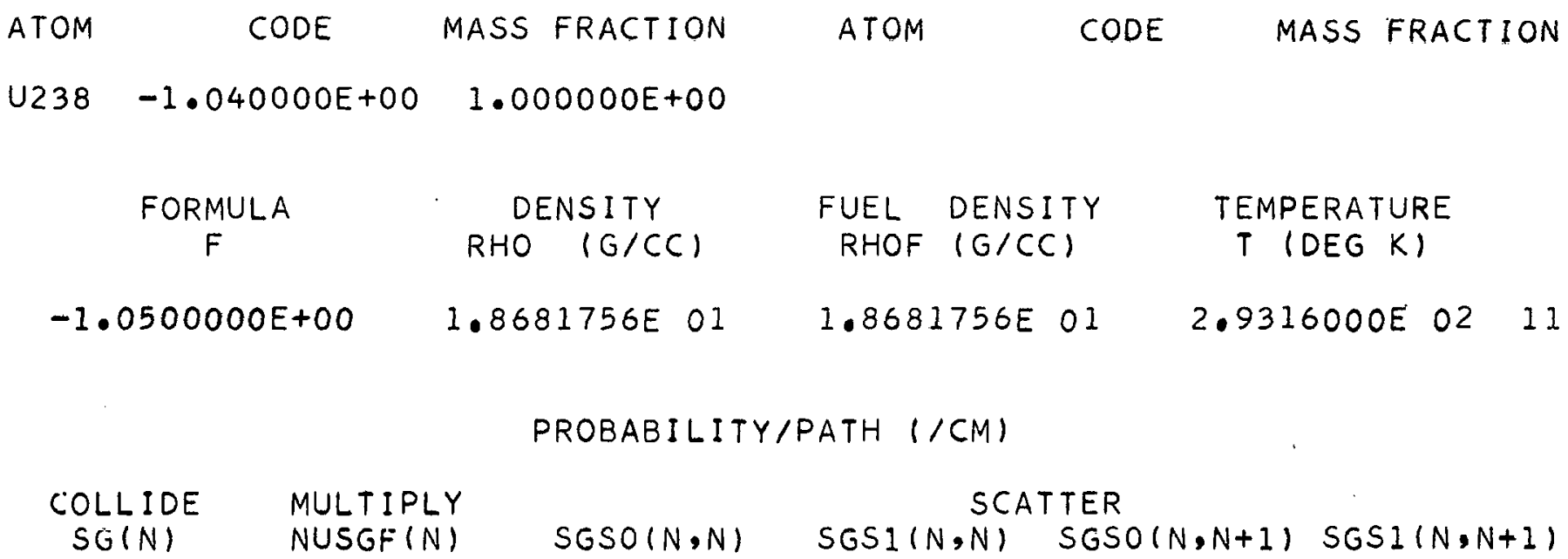

$\begin{array}{lllll}3.3441 E-01 & 4.5021 E-02 & 3.1101 E-01 & 1.9959 E-03 & 2.8337 E-03-1.5232 E-03 \\ 3.2944 E-01 & 1.4771 E-02 & 3.1480 E-01 & 2.3693 E-03 & 4.2458 E-03-1.6603 E-03 \\ 3.6088 E-01 & 0 . & 3.4884 E-01 & 2.9634 E-03 & 5.6577 E-03-2.0180 E-03 \\ 4.2492 E-01 & 0 . & 4.1005 E-01 & 3.8265 E-03 & 7.0718 E-03-2.6449 E-03 \\ 4.8708 E-01 & 0 . & 4.6938 E-01 & 4.4824 E-03 & 8.4844 E-03-3.0644 E-03 \\ 5.8161 E-01 & 0 . & 5.6401 E-01 & 2.5635 E-03 & 2.4748 E-03-9.0914 E-04 \\ 6.5653 E-01 & 0 . & 6.2108 E-01 & 2.9574 E-03 & 2.8283 E-03-1.0667 E-03 \\ 7.1443 E-01 & 0 . & 6.2108 E-01 & 2.9574 E-03 & 2.8283 E-03-1.0667 E-03 \\ 1.1599 E-00 & 0 . & 5.6944 E-01 & 2.5574 E-03 & 2.4748 E-03-9.0309 E-04 \\ 7.1443 E-00 & 0 . & 4.7763 E-01 & 2.1751 E-03 & 2.1211 E-03-7.5711 E-04 \\ 6.5875 E-00 & 0 . & 4.3320 E-01 & 2.4210 E-03 & 2.8272 E-03-1.0030 E-03 \\ 4.4926 E-01 & 0 . & 4.2517 E-01 & 2.8872 E-03 & 4.2404 E-03-1.4692 E-03 \\ 4.5352 E-01 & 0 . & 4.1737 E-01 & 2.4435 E-03 & 3.5356 E-03-1.2619 E-03 \\ 4.6179 E-01 & 0 . & 4.0531 E-01 & 2.1224 E-03 & 2.8306 E-03-1.1770 E-03 \\ 4.8428 E-01 & 0 . & 3.9197 E-01 & 1.9096 E-03 & 2.6819 E-03-9.6423 E-04 \\ 4.8954 E-01 & 0 . & 3.7426 E-01 & 1.0493 E-03 & \end{array}$


FORMULA

$F$

\author{
DENS ITY \\ RHO $(G / C C)$
}

$1.8900008 \mathrm{E} \quad 01$
FUEL DENSITY RHOF $(G / C C)$

\section{TEMPERATURE \\ $T$ (DEG K)}

$1.8900008 E \quad 01 \quad 2.9316000 E \quad 02 \quad 11$

COLLIDE MULTIPLY $S G(N)$
MUL TIPLY
NUSGF $(N)$

PROBABILITY/PATH (/CM)

SCATTER
SGSO $(N, N) \quad S G S I(N, N) \quad \operatorname{SGSO}(N, N+1)$ SGSI $(N, N+1)$

$\begin{array}{llllll}3.3754 E-01 & 4.6675 E-02 & 3.1350 E-01 & 2.0144 E-03 & 2.8651 E-03-1.5365 E-03 \\ 3.3269 E-01 & 1.6129 E-02 & 3.1743 E-01 & 2.3905 E-03 & 4.2855 E-03-1.6749 E-03 \\ 3.6435 E-01 & 1.2436 E-03 & 3.5166 E-C 1 & 2.9879 E-03 & 5.7046 E-03-2.0348 E-03 \\ 4.2898 E-01 & 1.3090 E-03 & 4.1339 E-01 & 3.8571 E-03 & 7.1290 E-03-2.6659 E-03 \\ 4.9191 E-01 & 1.3939 E-03 & 4.7342 E-01 & 4.5198 E-03 & 8.5554 E-03-3.0900 E-03 \\ 5.8769 E-01 & 1.9093 E-03 & 5.0909 E-01 & 2.5857 E-03 & 2.4966 E-03-9.1685 E-04 \\ 6.6397 E-01 & 3.1295 E-03 & 5.2678 E-01 & 2.9830 E-03 & 2.8532 E-03-1.0757 E-03 \\ 7.2407 E-01 & 6.1916 E-03 & 6.2678 E-01 & 2.9830 E-03 & 2.8532 E-03-1.0757 E-03 \\ 1.1818 E-00 & 2.0477 E-02 & 5.7491 E-01 & 2.5812 E-03 & 2.4982 E-03-0.1132 E-04 \\ 7.2103 E-00 & 4.5166 E-02 & 4.3268 E-01 & 2.1972 E-03 & 2.1428 E-03-7.6470 E-04 \\ 6.6465 E-0 & 3.8768 E-02 & 4.3806 E-01 & 2.4456 E-03 & 2.8563 E-03-1.0131 E-03 \\ 4.7605 E-01 & 4.2235 E-02 & 4.2998 E-01 & 2.9167 E-03 & 4.2839 E-03-1.4842 E-03 \\ 5.0859 E-01 & 1.0195 E-01 & 4.2214 E-01 & 2.4711 E-03 & 3.5760 E-03-1.2759 E-03 \\ 5.5313 E-01 & 1.7744 E-01 & 4.1003 E-01 & 2.1485 E-03 & 2.8679 E-03-1.1907 E-03 \\ 6.4788 E-01 & 3.3074 E-01 & 3.9671 E-01 & 1.9345 E-03 & 2.7178 E-03-0.7653 E-04 \\ 7.0285 E-01 & 4.2894 E-01 & 3.7887 E-01 & 1.0622 E-03 & \end{array}$


ATOM CODE MASS FRACTION ATOM CODE MASS FRACTION

PU239-1.200000E+00 1.000000E+00

$\begin{array}{cccccc}\text { FORMULA } & \text { DENSITY } & \text { FUEL } & \text { DENSITY } & \text { TEMPERATURE } \\ F & \text { RHO }(G / C C) & \text { RHOF }(G / C C) & \text { T (DEG K) } \\ -1.2100000 E+00 & 1.9599999 E 01 & 1.9599999 E 01 & 2.9316000 E 02 & 11\end{array}$

PROBABILITY/PATH (/CM)

COLLIDE MULTIPLY

SG(N) NUSGF(N)
SCATTER
SGSO $(N, N) \quad S G S I(N, N) \quad S G S O(N, N+1)$ SGSI $(N, N+1)$

\begin{tabular}{|c|c|c|c|c|c|}
\hline $\begin{array}{l}3.4814 E-01 \\
3.4814 E-01\end{array}$ & $\begin{array}{l}2.8444 E-01 \\
2.6320 E-01\end{array}$ & $\begin{array}{l}2.0636 E-01 \\
2.1121 E-01\end{array}$ & $\begin{array}{l}1.7974 E-03 \\
1.8596 E-03\end{array}$ & $\begin{array}{l}3.5163 E-03 \\
3.5991 E-03\end{array}$ & $\begin{array}{l}-1.2 \\
-1.2\end{array}$ \\
\hline $\begin{array}{l}5 E-01 \\
37 E-01 \\
+7 E-01 \\
3 E-01\end{array}$ & $\begin{array}{l}2.4197 E-01 \\
2.3481 E-01 \\
2.2765 E-01 \\
4.3382 E-01 \\
8.1776 E-01\end{array}$ & $\begin{array}{l}2 . \\
3 . \\
3 . \\
3 .\end{array}$ & $\begin{array}{l}2.44 \\
2.99 \\
3.12 \\
1.68 \\
1.68\end{array}$ & $\begin{array}{l}37 E-03 \\
80 E-03 \\
36 E-03 \\
48 E-03 \\
+8 E-03\end{array}$ & $\begin{array}{l}-1 \\
-2 \\
-2 \\
-5 \\
-5\end{array}$ \\
\hline $\begin{array}{l}6.7900 E-01 \\
1.7530 E \text { OO } \\
3.1604 E \text { OO } \\
3.3086 E \text { OO } \\
2.9012 E \text { OI }\end{array}$ & 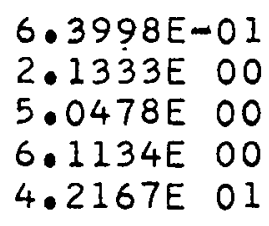 & $\begin{array}{l}40 E-01 \\
40 E-01 \\
40 E-01 \\
85 E-01 \\
74 E-01\end{array}$ & $\begin{array}{l}1.6843 E-03 \\
1.6843 E-03 \\
1.6843 E-03 \\
1.8771 E-03 \\
2.2625 E-03\end{array}$ & $\begin{array}{r}1.6548 E-03 \\
1.6548 E-03 \\
1.6548 E-03 \\
2.2064 E-03 \\
3.3095 E-03\end{array}$ & $\begin{array}{l}-5 \cdot 78 \\
-5 \cdot 78 \\
-5 \cdot 78 \\
-7 \cdot 70 \\
-1 \cdot 15\end{array}$ \\
\hline $\begin{array}{ll}6.2739 E & 01 \\
6.0557 E & 01 \\
3.9488 E & 01 \\
4.9910 E & 01\end{array}$ & $\begin{array}{ll}1.0027 E & 02 \\
1.0844 E & 02 \\
8.0385 E & 01 \\
1.0243 E & 02\end{array}$ & $\begin{array}{l}3.9174 E-01 \\
4.3092 E-01 \\
4.7030 E-01 \\
4.9360 E-01\end{array}$ & $\begin{array}{l}.2625 E-03 \\
.5518 E-03 \\
.6297 E-03 \\
.3821 E-03\end{array}$ & $\begin{array}{l}E-03 \\
E-03 \\
E-03\end{array}$ & $\begin{array}{l}-1 \\
-1 \\
-1\end{array}$ \\
\hline
\end{tabular}

$3.4814 E-01$ $3.4814 E-01$

3.9505E-01

4.8147E-01

$6.1603 E-01$

$6.7900 E-01$

$1.7530 E$ OO

$3.1604 \mathrm{E} 00$

$3.3086 E \quad 00$

$6.2739 \mathrm{E} 01$

$6.0557 E$ OI

4.9910E OI
$2.8444 E-01$

$.4197 E-01$

$3481 E-01$

.

$1776 \mathrm{E}-01$

$6.3998 E-01$

2.1333E 00

$.0478 E \quad 00$

$6.1134 \mathrm{E} \quad 00$

$1.0027 \mathrm{E} 02$

$1.0844 \mathrm{E} \quad 02$

$1.0243 E \quad 02$
$3.5163 E-03-1.2097 E-03$

4.6337E-03-1.6704E-03

$5.6680 E-03-2.0460 E-03$

$6.1636 E-03-2.0904 E-03$

$1.6548 E-03-5.7819 E-04$

$1.6548 E-03-5.7819 E-04$

$1.6548 E-03-5.7819 E-04$

$1.6548 E-03-5.7819 E-04$

$2.2064 E-03-7.7092 E-04$

$3.3095 E-03-1.1564 E-03$

$3.3095 E-03-1.1564 E-03$

$3.764 I E-03-I .3023 E-03$ 


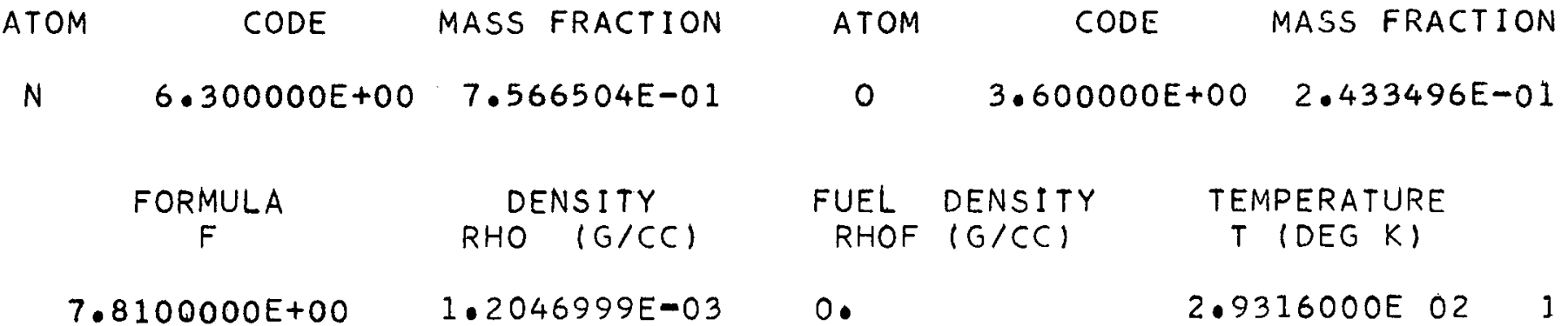

PROBABILITY/PATH (/CM)

$\begin{array}{cc}\text { COLLIDE } & \text { MUL } \\ \text { SG }(N) & \text { NU } \\ 8.0625 E-05 & 0 . \\ 9.0743 E-05 & 0 . \\ 8.6065 E-05 & 0 . \\ 9.6225 E-05 & 0 . \\ 1.1391 E-04 & 0 . \\ & \\ 1.3013 E-04 & 0 . \\ 1.5746 E-04 & 0 . \\ 1.8157 E-04 & 0 . \\ 2.2374 E-04 & 0 . \\ 2.6009 E-04 & 0 . \\ & \\ 3.3658 E-04 & 0 . \\ 4.1877 E-04 & 0 . \\ 4.3444 E-04 & 0 . \\ 4.3909 E-04 & 0 . \\ 4.4635 E-04 & 0 . \\ 4.5438 E-04 & 0 . \\ 4.6992 E-04 & 0 . \\ 5.0601 E-04 & 0 . \\ 5.3997 E-04 & 0 .\end{array}$

\section{SGSO $(N, N)$}

$5.8726 E-05$

$6.6416 E-05$

$6.3029 E-05$

$7.0474 E-05$

$8.4075 E-05$

$9.6080 E-05$

$1.1561 \mathrm{E}-04$

$1.3304 E-04$

$2.0867 E-04$

$2.4244 E-04$

3. $1346 E-04$

3. $8941 E-04$

4.0237E-04

$3.9403 E-04$

$3.7611 E-04$

3. $7679 E-04$

$3.7991 E-04$

3.9669E-04 $4 \cdot 7460 E-04$
SCATTER

.SGSI $(N, N) \quad S G S O(N, N+1) \quad \operatorname{SGSI}(N, N+1)$

$1.0414 E-05$

$1.1575 E-05$

$1.0961 \mathrm{E}-05$

$1.2253 E-05$

$1.4210 E-05$

$2.1895 E-05-6.5889 E-06$

$2.4322 E-0.5-7.3290 E-06$

$2.3029 E-05-6.9408 E-06$

$2.5743 E-05-7.7587 E-06$

$2.9823 E-05-9.0090 E-06$

$1.6217 E-05 \quad 3.4033 E-05-1.0282 E-05$

$1.9918 E-05$

$2.3085 E-05$

$1.5013 E-05$

$1.7502 E-05$

$4.1832 E-05-1.2618 E-05$

$4.8498 E-05-1.4619 E-05$

$1.5018 E-05-4.5246 E-06$

$1.7508 E-05-5.2731 E-06$

$2.2736 E-05$

$2.8333 \mathrm{E}-05$

$2.9289 E-05$

$3.2346 E-05$

$3.8421 E-05$

$2.2744 E-05-6.8468 E-06$

$2.8343 E-05-8.5298 E-06$

$2.9298 E-05-8.8170 E-06$

$3.9208 E-05-1.1799 E-05$

$5.9081 E-05-1.7780 E-05$

$3.8491 E-05$

$5.9189 E-05-1.7812 E-05$

3.8810E-05

$3.9217 E-05$

$5.9680 E-05-1.7960 E-05$

$5.8574 E-05-1.7627 E-05$ 


\begin{tabular}{|c|c|c|c|c|c|c|}
\hline ATOM & FORMULA & MASS FRACTION & ATOM & \multicolumn{2}{|c|}{ FORMULA } & MASS FRACTION \\
\hline$A I$ & $5.1000000 E+00$ & $5.2920000 E-01$ & 0 & \multicolumn{2}{|c|}{$3.6000000 E+00$} & $4.7080000 \mathrm{E}-01$ \\
\hline & $\begin{array}{c}\text { FORMULA } \\
F\end{array}$ & $\begin{array}{l}\text { DENSITY } \\
\text { RHO }(G / C C)\end{array}$ & $\begin{array}{l}\text { FUEL } \\
\text { RHOF }\end{array}$ & $\begin{array}{l}\text { DENSITY } \\
(G / C C)\end{array}$ & \multicolumn{2}{|c|}{$\begin{array}{l}\text { TEMPERATURE } \\
T \text { (DEG K) }\end{array}$} \\
\hline & & $3.9699999 E \quad 00$ & 0. & & 2.93 & $6000 E \quad 02101$ \\
\hline
\end{tabular}

\section{COLLIDE $S G(N)$ MUL TIPLY NUSGF $(N)$}

$1.3933 E-01$ $2.1814 E-01$ $2.5004 E-01$ 2.6130E-01 4. $0683 E-01$

$\begin{array}{ll}5.3138 E-01 & 0 . \\ 4.4741 E-01 & 0 . \\ 4.9726 E-01 & 0 . \\ 4.8764 E-01 & 0 . \\ 3.3167 E-01 & 0 . \\ 3.2725 E-01 & 0 . \\ 3.2734 E-01 & 0 . \\ 3.2736 E-01 & 0 . \\ 3.2946 E-01 & 0 . \\ 3.3305 E-01 & 0 . \\ 3.3434 E-01 & 0 . \\ 3.3621 E-01 & 0 . \\ 3.5149 E-01 & 0 . \\ 3.7167 E-01 & 0 .\end{array}$

\section{SCATTER}

SGSO $(N, N) \quad S G S I(N, N) \quad S G S O(N, N+I) \quad S G S I(N, N+I)$

\begin{tabular}{|c|c|c|c|}
\hline$=$ & $\begin{array}{l}2.5497 \mathrm{E}- \\
3.4480 \mathrm{E} \\
3.9664 \mathrm{E}- \\
4.1000 \mathrm{E}\end{array}$ & $\begin{array}{l}4 . \\
6 \\
7 . \\
7 \\
1 .\end{array}$ & $\begin{array}{l}9 \\
1 \\
2 \\
1\end{array}$ \\
\hline $\begin{array}{l}1 \\
1 \\
1\end{array}$ & $\begin{array}{l}7.9424 E-02 \\
6.8052 E-02 \\
4.7191 E-02 \\
2.4095 E-0 \\
1.8412 E-02\end{array}$ & $\begin{array}{l}1.5052 E-01 \\
1.3007 E-01 \\
9.7994 E-02 \\
2.4100 E-02 \\
1.8416 E-02\end{array}$ & $\begin{array}{r}1 \\
1 \\
-3 \\
-7 \\
-5\end{array}$ \\
\hline $\begin{array}{l}0894 E-01 \\
0894 E-01 \\
0871 E-01 \\
0419 E-01\end{array}$ & $\begin{array}{l}1.8249 E-02 \\
1.8249 E-02 \\
1.8241 E-02 \\
2.0169 E-02 \\
2.4042 E-02\end{array}$ & $\begin{array}{l}1.825 \\
1.824 \\
2.440 \\
3.681\end{array}$ & $\begin{array}{l}-5 \\
-7 \\
-1\end{array}$ \\
\hline$Y 482 E-C$ & $\begin{array}{l}2.4053 E-02 \\
2.4113 E-02 \\
2.4496 E-02\end{array}$ & $\begin{array}{l}3.6832 E-02 \\
3.6925 E-02 \\
3.6448 E-02\end{array}$ & -1 \\
\hline
\end{tabular}

RELIABILITY LIMITATION: FIRST-SIGNIFICANT-FIGURE UNCERTAINTY IN HIGH ENERGY SCATTER CROSS SECTIONS FOR ALUMTNUM CONTENT. 


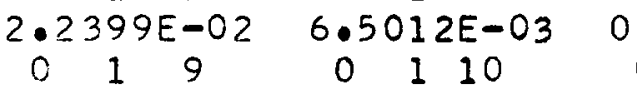
$\begin{array}{rrrrrr}0 & 1 & 9 & 0 & 1 & \\ 0 & 1 & 15 & 0 & 1 & 16\end{array}$

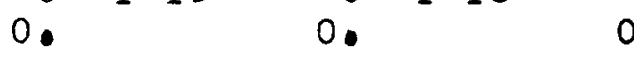
C 250226 $1.3406 E-02$ 0211

0. $217 \quad 0$

0.3217

$5.7272 E-03$

O 314

C. 46

$2.5845 E-02$

0412

0

0418

0.511

$\begin{array}{rrr}0 . & & 11 \\ 0 & 517\end{array}$

$\begin{array}{ccc}0 . & & \\ 0 & 6 & 11\end{array}$

$\begin{array}{rrr}0 . & & \\ 0 & 617\end{array}$

0.712

0.718

0.

0 .

0911

0.

$\begin{array}{lll}0 & 9 & 17\end{array}$

0.

01015

0 .

0 1114

$\begin{array}{rrr}0 & 1214\end{array}$

0 .

$0 \quad 1315$

0 .

$\begin{array}{lll}0 & 14 & 17\end{array}$

0 .

C $16 \quad 18$

0 .
0
$5.0066 E-03$

0212

0.218

0.39

4.6893E-C3

0315

0 .

$\begin{array}{lll}0 & 4 & 7\end{array}$

$1.5676 E-02$

0413

0.419

0.512

0.

$\begin{array}{lll}0 & 518\end{array}$

0.612

0.

0618

0. 713

$\begin{array}{rrr}0 & 7 & 19 \\ 0 . & & \\ 0 & 8 & 15\end{array}$

0

0912

0.
0

0.

01016

0 .

01115

C. 1215

$\begin{array}{rrr}0 & 12 & 15 \\ 0 & 13 & 16\end{array}$

0 .

01418

0.

01619

0.
0.719
111

117

0 .

$\begin{array}{lll}0 & 2\end{array}$

4. 722 7E-06

0213

0.219

0.310

0.

D 316

0 .

048

$9.5077 E-03$

0414

0.157

3. $0256 E-02$

0513

0.

0519

0 .

0613

0 .

0619

C. 714

0.810

0.
0.816

0 .

0913

0.019

0.

$0 \quad 10 \quad 17$

0 .

01116

0.1216

0 .

$\begin{array}{lll}0 & 13 & 17\end{array}$

0 .

01419

0 .

01719
0.
0
0.
0
0.
0
0.
0

0214

0.35

2. 5668E-02

0311

0 .

0

0.

317

049

$6.0552 E-03$

0415

0.58

$4 \cdot 3341 E-03$

0

068

4. $2963 E-02$

o 614

0.179

$6.6445 E-02$

0715

$\begin{array}{rrr}0 . & & \\ 0 & 811\end{array}$

0.817

$\begin{array}{ccc}0 & 817 \\ 0 . & & \end{array}$

0914

0 .

$0 \quad 1012$

0.
0

$\begin{array}{rrr}0 . & & \\ 0 & 1117\end{array}$

0.1217

0.1318

0 .

$\begin{array}{lll}0 & 15 & 17\end{array}$

0 . $\begin{array}{rrrrrr}0 & 1 & 7 & 0 & 1 & 8 \\ 0 & 0 & 1 & 0 & 0 & \\ 0 & 13 & 0 & 1 & 14\end{array}$

$\begin{array}{rrrrrr}0 . & 119 & 0.1 & & \\ 0 & 1 & 19 & 0 & 2 & 4\end{array}$

$0 . \quad 2.2103 E-02$

02902210

0.0

$\begin{array}{lllll}0 & 215 & 0 & 216\end{array}$

$0 . \quad 0$.

$\begin{array}{llllll}0 & 3 & 6 & 0 & 3 & 7\end{array}$

$1.5568 E-02 \quad 9.4426 E-03$

$\begin{array}{llllll}0 & 312 & 0 & 313\end{array}$

0.

0318

0.

0

0.

0

410

416

0 .

0319

0 .

0411

0 .

0417

0 .

0

59

$0 \cdot$

0510

0

515

069

$3.9195 E-02$

0.516

0 .

0610

0615

0616

0.

0710

0

0711

$\begin{array}{llll}0 & 716 & 0 & 0\end{array} 17$

0.812

0.813

0818

0 .

$0 \quad 819$

0.

0915

0

0916

0 .

$0 \quad 1013$

0.

01014

0.1019

0 .

01113

$0 . \quad 0$.

$01118 \quad 01119$

01218

0 .

$0 \quad 1219$

0.1319

0 .

$0 \quad 1416$

0 .

01518

0 .

01519 


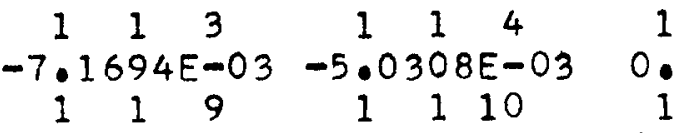

$$
\begin{array}{ccccccc}
1 & 1 & 9 & 1 & 1 & 10 & 1 \\
0 . & & & 0 & & & 0 .
\end{array}
$$

$\begin{array}{rrrrrrr}1 & 1 & 15 & 1 & 1 & 16 & 0\end{array}$

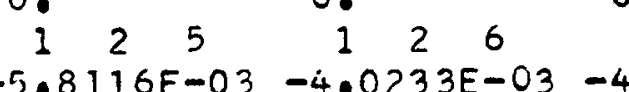

$\begin{array}{cccc}-5.8116 E-03 & -4.0233 E-03 & -4 \\ 1 & 211 & 1 & 212\end{array}$

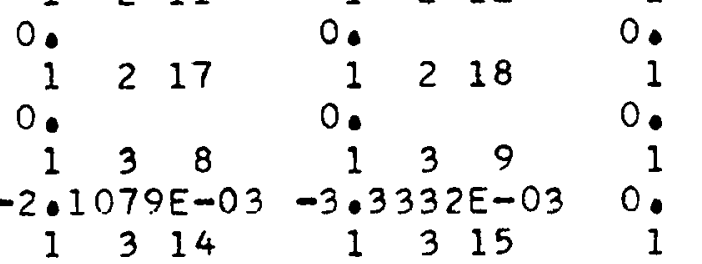

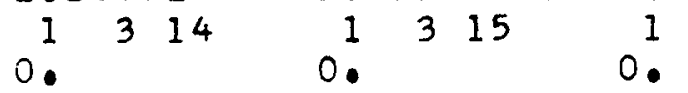

146

147

1

$7.4646 E-03$
1412

? 418

0.511

1517

0.611

$\begin{array}{lll}1 & 6 & 11\end{array}$

1617

1712

0. 718

0.1814

0.911

0.
1

$0: 10 \quad 15$

11114

$0: 1214$

0.

11315

1 1417

$\begin{array}{rll}0.16 & 18\end{array}$

0.
$-7.5913 E-04$ 1

1419

0.

1512

0. 518

0.

1612

0.618

0

1713

0.719

1 815

0.912

0.

1918

0.

11016

0.

11115

0.

11215

11316

0.

11418

0.1619

0.
0.

0.

0

0.

0.

0.

0.

0.

0. $\begin{array}{rrr}1 & 1 & 6 \\ 0 & 1 & 1 \\ 0 & 12 \\ 1 & 1 & 18\end{array}$

128

0.

1214

0

135

$1.0564 E-02$

1311

0.

1317

149

$\begin{array}{ccc}1.6959 E-03 & -4.5533 E-03 \\ 1414 & 1 & 415\end{array}$

0 .

158

$-1.3417 E-02-3.6405 E-03$

$\begin{array}{lll}1 & 5 & 14\end{array}$

0

168

$4.2187 E-03$

$\begin{array}{rrr}1 & 1 & 7 \\ 0 & 1 & 1 \\ 0 & 13 \\ 1 & 1 & 19\end{array}$

$\begin{array}{lll}1 & 1 & 8\end{array}$ 0.

1114

129

0.

1215

0.0

$\begin{array}{llllll}1 & 3 & 6 & 1 & 3\end{array}$

$2 \cdot 3000 E-03$

1312

0

0.410

124

$1.0585 E-03$

1210

0.

1216

$.0085 E-03$

1313

1319

1411

0.

1416

0.

159

0

1515

0.

169

1613

1619

0 .

$1.9191 E-02$

0.

1417

0

1510

179

-1.7817 E-02

1715

1 811

0 .

1

0.914

0 .

11012

0.

i 710

0.

1716

0 - 12

0

1516

1610

0.

1616

0.

1711

1717

1810

$\begin{array}{rrr}0 & 8 & 16\end{array}$

0.913

0.19

$\begin{array}{lll}1 & 10 & 17\end{array}$

0.

11018

$\begin{array}{lll}1 & 11 & 16\end{array}$

i 1216

$\begin{array}{lll}0.111 & 11\end{array}$

0 .

11317

11419

$\begin{array}{lll}1 & 12 & 17\end{array}$

0 .

11318

0.

11517

0.

1818

0.915

0.

11013

0.

11019

0.

11118

0.

11218

1813

0.

1819

1916

0.

11014

0.

11113

0.

11119

0.

0.

11319

11219

11416

0. 1518

0 .

11519

0 .

0.

11719 


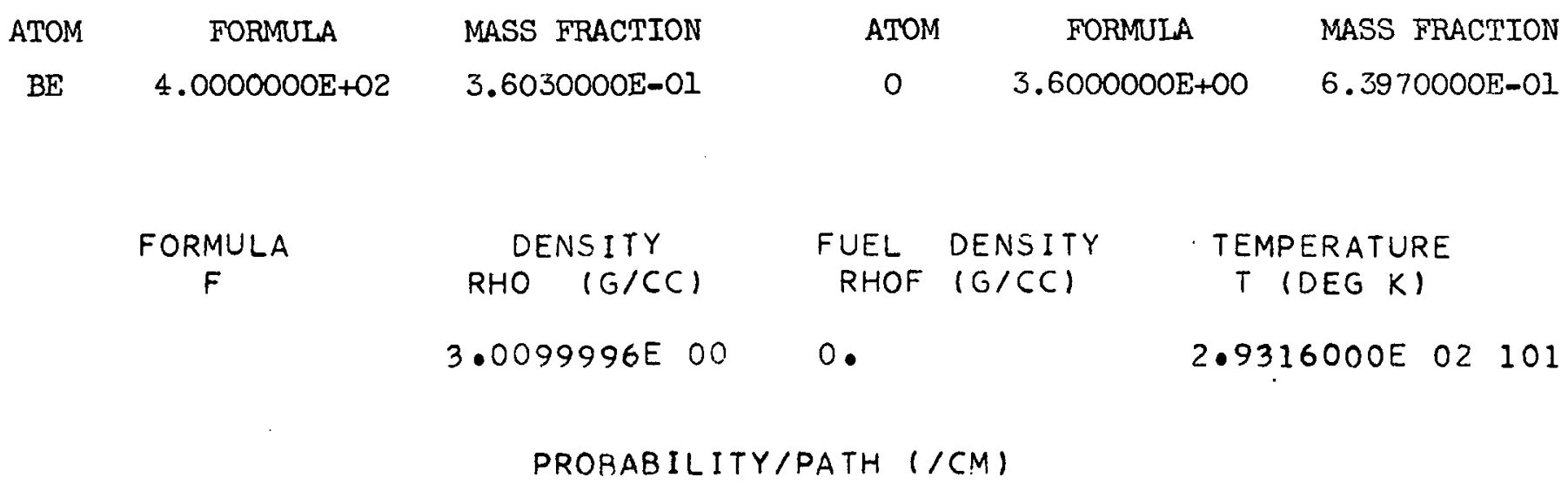

$\begin{array}{cc}\text { COLLIDE } & \text { MU } \\ \text { SG(N) } & \text { NUS } \\ 1.7694 E-01 & 0 . \\ 2.6315 E-01 & 0 . \\ 3.0587 E-01 & 0 . \\ 2.7325 E-01 & 0 . \\ 5.2085 E-01 & 0 . \\ 6.2588 E-01 & 0 . \\ 5.5796 E-01 & 0 . \\ 5.9891 E-01 & 0 . \\ 6.8270 E-01 & 0 . \\ 7.0668 E-01 & 0 . \\ 7.0669 E-01 & 0 . \\ 7.0669 E-01 & .0 . \\ 7.0671 E-01 & 0 . \\ 7.0674 E-01 & 0 . \\ 7.0679 E-01 & 0 . \\ 7.0071 E-01 & 0 . \\ 6.6712 E-01 & 0 . \\ 6.8053 E-01 & 0 . \\ 7.2857 E-01 & 0 . \\ & \end{array}$

SCATTER

SGSO (N,N) SGSI(N,N) SGSO(N,N+1) SGSI(N,N+1)

$1.1037 E-012.5694 E-02$

$1.5875 E-01 \quad 3.8894 E-02$

2.0252E-01 4.2186E-02

$1.9808 E-01 \quad 4.3947 E-02$

$3.6502 E-01 \quad 7.8508 E-02$

4.2724E-01

$3.7479 E-01$

3. $9713 E-01$

6. $0347 E-01$

$6.4503 E-01$

$6.4503 E-01$

$6.4503 E-01$

$6.4503 E-01$

$6.2448 E-01$

$5.8338 E-01$

$5.7850 E-01$

$5.5196 E-0.1$

$5.6968 E-01$

$7.2791 E-01$
$9.4817 \mathrm{E}-02$

$8.6081 E-02$

$9.3016 E-02$

$5.6733 E-02$

$6.1546 E-02$

$6.1546 E-02$

$6.1546 E-02$

$6.1546 \mathrm{E}-02$

$6.7457 E-02$

$7.9278 E-02$

$2.4483 E-02-1.5848 E-02$

$5.9432 E-02-2.4125 E-02$

$7.8699 E-02-2.6141 E-02$

$7.2514 E-02-2.7262 E-02$

$1.5550 E-01-4.8844 E-02$

$1.9864 E-01-5.8955 E-02$

$1.8317 E-01-5.3442 E-02$

$2.0178 E-01-5.7652 E-02$

$5.6826 E-02-1.6375 E-.02$

$6.1653 E-02-1.7732 E-02$

$6.1653 E-02-1.7732 E-02$

$6.1653 E-02-1.7732 E-02$

6.1653E-02 -1.7732E-02

8. $2204 E-02-2.3643 E-02$

$1.2331 E-01-3.5465 E-02$

$7.8459 E-02 \quad 1.2202 E-01-3.5104 E-02$

$7.3877 E-02$ 1.1486E-01 $-3.3092 E-02$

$7.3143 E-02$ i. $1034 E-01-3.1818 E-02$ 


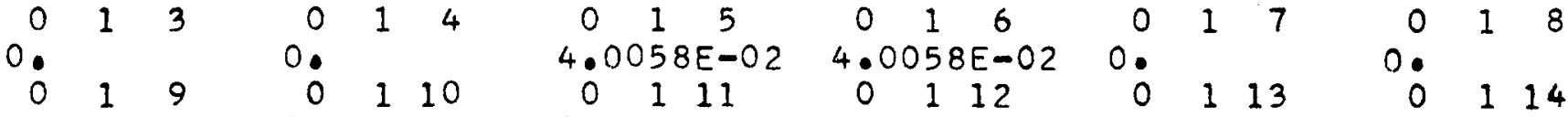

$\begin{array}{rlllll}0 & 15 & 0 & 0 & 15\end{array}$

$\begin{array}{rrrrr}0 . & & 0 . & & \\ 0 & 2 & 5 & 0 & 2\end{array}$

4.0312E-02 4.0312E-C2

0211

0212

0 .

0 .

0217

0218

0.13

$2.3410, E-03$

0.

0

$\begin{array}{llll}0 & 34 & 0\end{array}$

0.060

$\begin{array}{rrrr}0 & 4 & 6 & 0 \\ 0 & 4 & 12 & 0\end{array}$

0 .

0

0.

$\begin{array}{rrr}0 & 511 \\ 0 . & & \end{array}$

$\begin{array}{lll}0 & 517\end{array}$

0.611

0.617

$\begin{array}{rrr}0 . & & \\ 0 & 7 & 12\end{array}$

$\begin{array}{rll}0 . & & \\ 0 & 7 & 18\end{array}$

0.
0

0.
0

0.917

$\begin{array}{rrr}0 . & & \\ 0 & 10 & 15\end{array}$

0.1114

0. 1214

0.1315

0.1317
$0 \quad 1417$

0.1618

0 .
0315

047

- 413

0 .

0419

$\begin{array}{rll}0 . & & \\ 0 & 512\end{array}$

0.518

$\begin{array}{ccc}0 . & & \\ 0 & 612\end{array}$

$\begin{array}{rll}0 . & & 12 \\ 0 & 6 & 18\end{array}$

0

- 713

$\begin{array}{rrr}0 . & & \\ 0 & 719\end{array}$

0.
0

0.912

0.

0.1016

0.1115

0.1215

0.1316

0.1418

$\begin{array}{rrr}0 . & & \\ 0 & 1619\end{array}$

0 .
0918
0.

$0 . \quad 17$

027

0

0.

213

- 219

0.

0310

0.316

$\begin{array}{rrr}0 . & & \\ 0 & 4 & 8\end{array}$

0.

0

414

0 .

0513

0.519

0 .

0613

0.

0

0.

0

0

0

0.

0

0.

0

$0 \quad 1017$

0 .

01116

0.

01216

$\begin{array}{rrr}0 . & & \\ 0 & 1317\end{array}$

0

$014 \quad 19$

0.1719
0 57
0.

- 118

0.

0

0.

0

0 .

$2.2178 E-03$

0311

0.

0

049

0 .

0415

0.58

0 .

0514

$\begin{array}{rll}0 . & & \\ 0 & 6 & 8\end{array}$

0 .

0614

0.979

0 .

0715

0.811

0.

C 817

0.914

0 .

$0 \quad 1012$

u.

ว 1018

0.01117

0 .

$\begin{array}{lll}0 & 12 & 17\end{array}$

0.1318

0 .

$\begin{array}{lll}0 & 15 & 17\end{array}$

0 .
0.

0.

119

029

0215

0 .

036

0312

0.

0318

0 .

0

0.

0

059

0.

$0 \quad 515$

0.

069

0.

0 .

0710

0 .

0716

0.812

0 .

0

0 .

0

0 .

01013

0 .

$0 \quad 1019$

0 .

01118

0 .

01218

0 .

01319

0 .

01518

0 .
2. 578OE-O2
024

0.210

0.216

0 . 37

$4.4646 E-03$

0313

0

0319

0411

0.417

0.510

$\begin{array}{rll}0 . & & \\ 0 & 516\end{array}$

0.
0

0.616

0711

$\begin{array}{rrr}0 . & & \\ 0 & 7 & 17\end{array}$

0.
0

0819

0.916

- 1014

0.1113

0.1113

01119

0 .

01219

01416

01519 


\begin{tabular}{|c|c|c|c|c|c|c|}
\hline ATOM & FORMULA & MASS FRACTION & ATOM & \multicolumn{2}{|c|}{ FORMULA } & MASS FRACTION \\
\hline $\begin{array}{l}\text { AL } \\
\text { C }\end{array}$ & $\begin{array}{l}5.1000000 \mathrm{E}+00 \\
3.300000 \mathrm{E}+00\end{array}$ & $\begin{array}{l}8.0000000 \mathrm{H}-\mathrm{O} 1 \\
4.300000 \mathrm{~F}-\mathrm{O} 2\end{array}$ & B & \multicolumn{2}{|c|}{$8.0000000 \mathrm{E}+00$} & $1.5700000 \mathrm{E}-01$ \\
\hline & \multirow[t]{2}{*}{$\begin{array}{c}\text { FORMULA } \\
F\end{array}$} & $\begin{array}{c}\text { DENSITY } \\
\text { RHO }(G / C C)\end{array}$ & $\begin{array}{l}\text { FUEL } \\
\text { RHOF }\end{array}$ & $\begin{array}{l}\text { DENSITY } \\
(G / C C)\end{array}$ & \multicolumn{2}{|c|}{$\begin{array}{l}\text { TEMPERATURE } \\
T(D E G \quad K)\end{array}$} \\
\hline & & $2.5300003 E \quad 00$ & 0. & & $2 \cdot 93$ & $6000 E \quad 02101$ \\
\hline
\end{tabular}

PROBABILITY/PATH (/CM)

\begin{tabular}{|c|c|c|c|c|c|}
\hline \multirow{2}{*}{$\begin{array}{l}\text { COLLIDE } \\
\text { SG(N) }\end{array}$} & \multirow{2}{*}{$\begin{array}{l}\text { MULTIPLY } \\
\text { NUSGF }(N)\end{array}$} & \multicolumn{4}{|c|}{ SCATTER } \\
\hline & & $S G S O(N, N)$ & $\operatorname{SGSI}(N, N)$ & $\operatorname{SGSO}(N, N+1)$ & $\operatorname{SGSI}(N, N+I)$ \\
\hline $\begin{array}{l}1.8534 E-01 \\
1.9605 E-01 \\
2.2782 E-01 \\
2.2584 E-01 \\
2.2462 E-01\end{array}$ & $\begin{array}{l}0 . \\
0 . \\
0 . \\
0 . \\
0 .\end{array}$ & $\begin{array}{l}8.7342 E-02 \\
8.7110 E-02 \\
9.2401 E-02 \\
9.3246 E-02 \\
1.0210 E-01\end{array}$ & $\begin{array}{l}3.4902 E-02 \\
3.5991 E-02 \\
4.1134 E-02 \\
4.0918 E-02 \\
4.3269 E-02\end{array}$ & $\begin{array}{l}6.9192 E-02 \\
6.8671 E-02 \\
7.4983 E-02 \\
7.5568 E-0 ? \\
8.6594 E-0 ?\end{array}$ & $\begin{array}{l}3.1197 E-03 \\
8.9212 E-0.3 \\
1.8631 E-02 \\
1.6398 E-02 \\
4.2881 E-03\end{array}$ \\
\hline $\begin{array}{l}3.0326 E-01 \\
2.8090 E-01 \\
3.4074 E-01 \\
3.3987 E-01 \\
2.1075 E-01\end{array}$ & $\begin{array}{l}0 . \\
0 . \\
0 . \\
0 .\end{array}$ & $\begin{array}{l}1.1609 E-01 \\
1.1349 E-01 \\
2.6430 E-01 \\
3.1080 E-01 \\
1.6725 E-01\end{array}$ & $\begin{array}{l}5.6848 E-02 \\
5.2644 E-02 \\
3.3647 E-02 \\
1.7530 E-02 \\
1.2163 E-02\end{array}$ & $\begin{array}{l}1.0468 E-01 \\
9.9105 E-02 \\
7.0927 E-02 \\
1.7536 E-0 ? \\
1.2165 E-0 ?\end{array}$ & $\begin{array}{r}2.7303 E-02 \\
1.9709 E-02 \\
-2 \cdot 1494 E-02 \\
-5.3085 E-03 \\
-3.6073 E-03\end{array}$ \\
\hline $\begin{array}{l}2.6034 E-01 \\
4.0704 E-01 \\
8.0482 E-01 \\
1.5034 E \text { OO } \\
2.7095 E 00\end{array}$ & $\begin{array}{l}0 . \\
0 . \\
0 . \\
0 .\end{array}$ & $\begin{array}{l}1.6311 E-01 \\
1.6311 E-01 \\
1.6289 E-01 \\
1.6064 E-01 \\
1.5533 E-01\end{array}$ & $\begin{array}{l}1.2007 E-0 ? \\
1.2007 E-02 \\
1.1999 E-02 \\
1.3271 E-02 \\
1.5816 E-02\end{array}$ & $\begin{array}{l}1.2012 \mathrm{E}-0 ? \\
1.2012 \mathrm{E}-0 ? \\
1.2004 \mathrm{E}-02 \\
1.6111 \mathrm{E}-0 ? \\
2.4408 \mathrm{E}-02\end{array}$ & $\begin{array}{l}-3.5578 E-03 \\
-3.5578 E-02 \\
-3.5551 E-03 \\
-4.7731 E-03 \\
-7.7349 E-02\end{array}$ \\
\hline 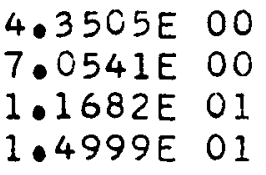 & $\begin{array}{l}0 . \\
0 . \\
0 .\end{array}$ & $\begin{array}{l}1.5554 E-01 \\
1.5407 E-01 \\
1.5469 E-01 \\
1.8066 E-01\end{array}$ & $\begin{array}{l}1.5827 E-02 \\
1.5751 E-02 \\
1.5355 E-02 \\
8.5831 E-03\end{array}$ & $\begin{array}{l}2.4425 E-02 \\
2.4209 E-02 \\
2 \cdot 3012 E-02\end{array}$ & $\begin{array}{l}-7.2401 E-03 \\
-7.2035 E-03 \\
-6.8178 E-03\end{array}$ \\
\hline
\end{tabular}

RELIABILITY LIMITATION: FIRST-SIGNIFICANT FIGURE UNCERTAINTY IN IIGH FNFRGY SCATIER CROSS SECTIONS OF ALUUINUM CONTENT. 


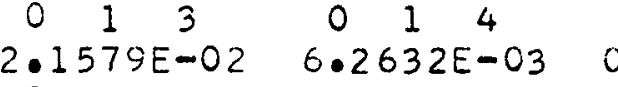

0

0

025

$1.2915 E-02$

0211

0.

0.

217

038

5.5I75E-03

O 314

0 .

046

$2.4898 E-02$

0.412

0.

$0 \cdot 418$

0

O 511

0.517

0.

$\begin{array}{rrr}0 & 6 & 17\end{array}$

$\begin{array}{rrr}0 . & & \\ 0 & 7 & 12\end{array}$

0.

$\begin{array}{lll}0 & 7 & 18\end{array}$

0.

0

0911

0.
0

0.1015

0
0 $11 \quad 14$

0.1214

0.1315

$\begin{array}{cll}0 . & & \\ 0 & 14 & 17\end{array}$

$\begin{array}{rll}0 & 16 & 18\end{array}$

0.
0

0

026

$4.8233 E-C 3$

0212

0.

0

039

4.5176E-03

0315

$c$

$\begin{array}{lll}0 & 4\end{array}$

$1.5102 E-02$

0413 .

0

0419

0.512

0.
0 518

0 .

0612

0.618

0.
0

0. 719

$\begin{array}{rrr}0 . & & 19 \\ 0 & 8 & 15\end{array}$

$\begin{array}{rrr}0 . & & \\ 0 & 9 & 12\end{array}$

$\begin{array}{rll}0 . & & \\ 0 & 9 & 18\end{array}$

0.

01016

$0 \begin{array}{lll}0 & & \\ 0 & 11 & 15\end{array}$

0
0

$\begin{array}{rll}0 . & & \\ 0 & 1316\end{array}$

$\begin{array}{rll}0 . & & \\ 0 & 1418\end{array}$

0.1619

0 .
0

1

111

0.17

0 .

027

4. 5497E-06

0213

0.219

0.0310

$\mathrm{c}$.

0316

c.

048

$9 \cdot 1596 E-03$

0414

0.

$2.9148 E-02$

0513

0.519

0 .

0613

0.619

$\begin{array}{lll}0 . & & \\ 0 & 7 & 14\end{array}$

0.810

0.16

0.913

0.919

0. 1017

0. 1116

$0.12 \quad 16$

0.1317

0 .

01419

0 .

01719 $\begin{array}{rrr}0 & 1 & 6 \\ 0 . & & \\ 0 & 1 & 12 \\ 0 . & \\ 0 & 1 & 18 \\ 0 & & \\ 0 & 2 & 8\end{array}$

0 .

0214

0.35

$2.4728 E-02$

0311

0 .

0317

0 .

049

$5.8334 E-03$

C 415

0.

4. $1754 E-03$

0514

0

0 68

4.1390E-02

0614

0.79

$6.4012 E-02$

0715

0 .

- 811

0.817

0 .

0914

0.1012

0 .

01018

0 .

01117

; 1217

0

o 1318

0 .

- 1517

0 . $\begin{array}{rrr}0 & 1 & 7 \\ 0.0 & & \\ 0 & 1 & 13 \\ 0.0 & & \\ 0 & 1 & 19 \\ 0.0 & & \\ 0 & 2 & 9\end{array}$

0 .

0

0 0

$\begin{array}{llllll}0 & 3 & 6 & 0 & 3 & 7\end{array}$ $1.4998 E-02 \quad 9.0968 E-03$

03120313

0 .

0

0 .

0410

0 .

0416

0.

059

0.

0515

0

069

$3 \cdot 77605-0$
$0 \quad 515$

0 .

0710

0. 716

0.812

$\begin{array}{rrr}0 & 812 \\ 0 & 818\end{array}$

0

० 915

0.

01013

0 .

$0 \quad 1019$

0 .

$0 \begin{array}{lll}0 & 11 & 18\end{array}$

0 .

01218

0 .

01319

0 .

01518

0 . $\begin{array}{lll}0 & 1 & 8 \\ 0 . & & \end{array}$

0114

024

$2 \cdot 12945-02$

0210

0216

0.319

0
0

0 .

0417

0.
0

0 . 16

0516

0.610

0.

0616

0 .

0711

$\begin{array}{rrrr}0 . & & \\ 0 & 7 & 17\end{array}$

0.813

0.819

0.916

0 .

$0 \quad 1014$

0 .

0 1113

0.

01119

0 .

O 1416

0 .

01519
0.1219 
$\begin{array}{llllllllllllllllll}1 & 1 & 3 & 1 & 1 & 4 & 1 & 1 & 5 & 1 & 1 & 6 & 1 & 1 & 7 & 1 & 1 & 3\end{array}$ $\begin{array}{cccccccccccccc}-6.9069 E-03 & -4.8466 E-03 & 0 . & & 0.0 & & 0 . & & 0 . & 13\end{array}$ 0.0000000 $\begin{array}{rrrrrr}1 & 1 & 15 & 1 & 1 & 16 \\ 0 . & & 0 & & & 16\end{array}$ $\begin{array}{llllllllllll}1 & 2 & 5 & 1 & 2 & 6 & 1 & 2 & 7 & 1 & 2 & 8\end{array}$ $-5.5988 E-03-3.8760 F-03-4.5315 E-06 \quad 0$.

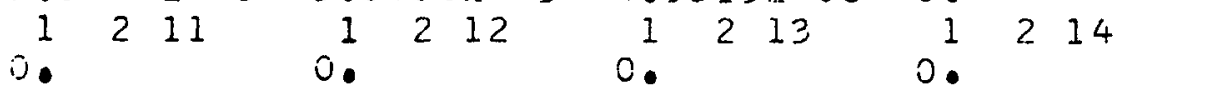

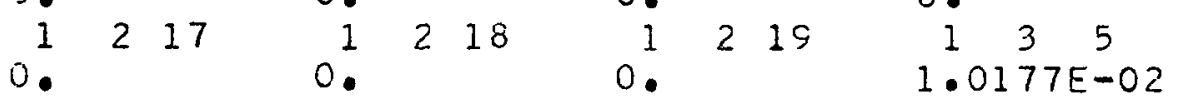
$\begin{array}{llllll}1 & 3 & 8 & 1 & 3 & 9\end{array}$ $-2.0308 E-03-3.2112 F-03$ $\begin{array}{rrrrrr}1 & 3 & 14 & 1 & 315 \\ 0 . & & 0 & & \end{array}$ 1310 $\begin{array}{rrrrrr}1 & 1 & 13 & 1 & 1 & 14 \\ 0 & 13 & 0 & & \end{array}$ $\begin{array}{llllll}1 & 1 & 19 & 1 & 2 & 4\end{array}$ $0.1 .0198 E-03$

$\begin{array}{lllllll}1 & 2 & 9 & 1 & 2 & 10\end{array}$

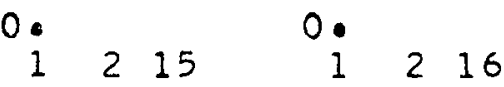
0.00 $\begin{array}{llllll}1 & 3 & 6 & 1 & 3 & 7\end{array}$ $\begin{array}{llllllllll}1 & 3 & 11 & 1 & 3 & 12 & 1 & 3 & 12\end{array}$ 0.000000 $\begin{array}{lllllllllllllll}1 & 3 & 16 & 1 & 3 & 17 & 1 & 3 & 18 & 1 & 3 & 19\end{array}$

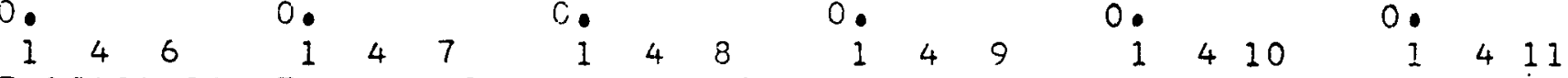
$7.1913 E-03-7.3134 E-04-3.5606 E-03-4.3866 E-03$

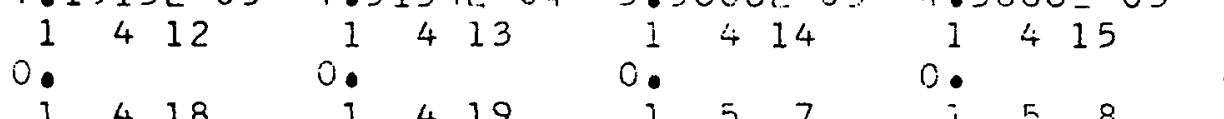

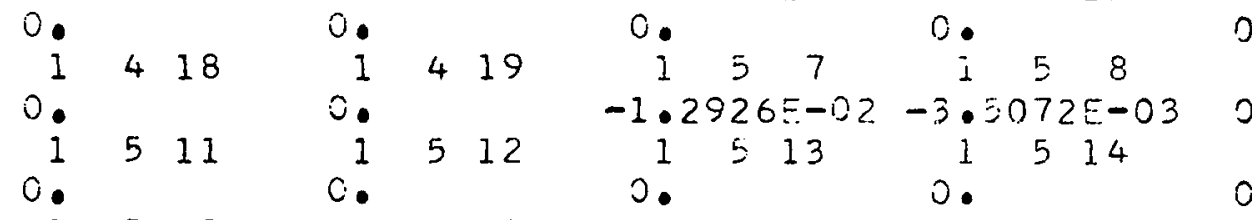
$\begin{array}{rrrrrr}1 & 5 & 17 & 1 & 5 & 18 \\ 0 & 1 & 6 & 11 & 0 & 1\end{array}$ 1519 $\begin{array}{llll}1 & 5 & 3 & 1\end{array}$ 0. $1416 \quad 1 \quad 417$ i 59 i 510 - 50.00 $1515 \quad 1516$ 1 0.

0.13

i 619 $\begin{array}{llllll}1 & 6 & 17 & 1 & 6 & 18\end{array}$

$\begin{array}{rrr}1 & 712\end{array}$

1718

1713

0.1814

i. 911

0. 917

$0: 1015$

0.1114

0.

11214

0. 1315

j. $14 \quad 17$

i) $16 \quad 18$
$171 \%$

c. 315

i. 912

1918

0. 1016

0.1115

1 1316

$\begin{array}{lll}1 & 14 & 18\end{array}$

$$
\text { i } 1619
$$

0.1215
0

0

0.

1810

0. 1616

0.913

$\begin{array}{rrr}0 . & & \\ 1 & 9 & 19\end{array}$

0.

i $10 \quad 17$

0.16

0 .

1. 1.216

0.1317

0.

11419

0 .

11719
$4.0542 E-03-1.8488 E-02$

$\begin{array}{llllll}1 & 6 & 14 & 1 & 6 & 15\end{array}$

i. 0 .

1791

$-1.7165 E-02$

$\begin{array}{llll}1 & 715 & 1\end{array}$

1811

0.

$\begin{array}{lll}1 & 8 & 17\end{array}$

0.1914

0 .

11012

$c$.

1.018

2.

11117

0.

11217

0.

11318

0.

$\begin{array}{lll}1 & 15 & 17 \\ 0 & & \end{array}$
1710

0

I 710

1812

0 .

1818

0 .

1

915

0.

11013

0.

$110 \quad 19$

0.

11118

0 .

11218

0.

11319

0.

11518

0.
1610 0.

1616 $\begin{array}{lll}0 & 71\end{array}$

0.

1717

0 .

18.13

0.

1819

0.

1916

0.

I $10 \quad 14$

0 .

11113

11119

0 .

11219

0.

11416

0.

11519 


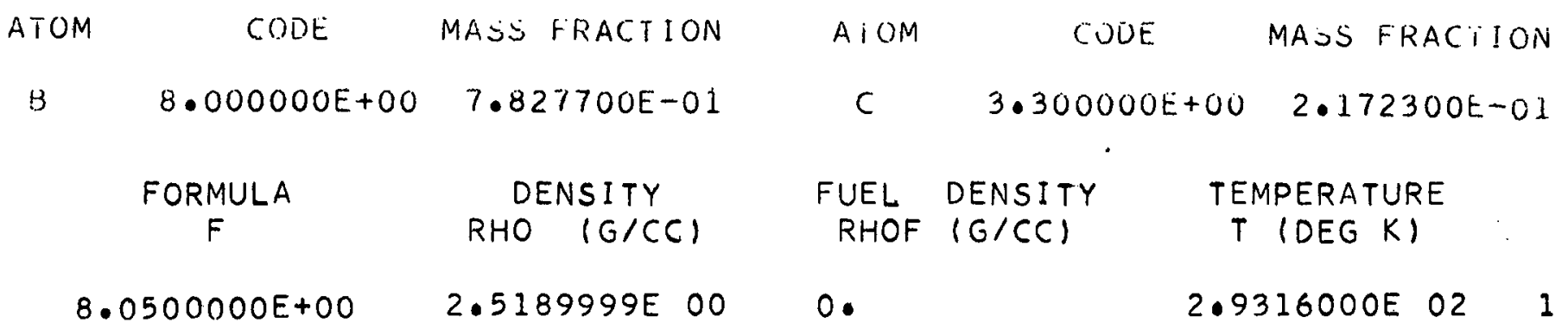

PROBABILITY/PATH (/CM)

\section{COLLIDE MULTIPLY $S G(N) \quad N U S G F(N)$}

$4.8303 E-01$

4. $8575 E-01$

$4.9776 E-01$

$5.0481 E-01$

5. $2139 E-01$

$5.4177 E-01$

$5.5995 E-01$

$5.7670 E-01$

$6.1852 E-01$

$7.2357 \mathrm{E}-01$

9.9090E-01

$1.7187 \mathrm{E} 00$

$3.6932 \mathrm{E} \quad 00$

$7.1512 E \quad 00$

$1.3121 \mathrm{E} 01$

$2.1261 E 01$

$3.4681 E$ OI

$5.7645 E \quad 01$

$7.4083 E 01$

\section{0. \\ 0. \\ 0. \\ 0 . \\ 0.}

0.

0.

0.

0.

0.

0.

0 .

0.

0.

0.

0.

0.

0.

0.

\begin{tabular}{|c|c|c|c|}
\hline \multicolumn{4}{|c|}{ SCATTER } \\
\hline $5 G S O(N, N)$ & $\operatorname{SGSI}(N, N)$ & $\operatorname{SGSO}(N, N+1)$ & $\operatorname{SGS1}(N, N+1)$ \\
\hline $\begin{array}{l}3.1193 E-01 \\
3.1286 E-01 \\
3.1988 E-01 \\
3.2316 E-01 \\
3.3252 E-01\end{array}$ & $\begin{array}{l}7.7864 E-02 \\
7.8069 E-02 \\
7.9610 E-02 \\
8.0330 E-02 \\
8.2385 E-02\end{array}$ & $\begin{array}{l}1.6634 E-01 \\
1.6678 E-01 \\
1.7005 E-01 \\
1.7158 E-01 \\
1.7594 E-01\end{array}$ & $\begin{array}{l}-4.8401 E-02 \\
-4.8530 E-02 \\
-4.9495 E-02 \\
-4.9945 E-02 \\
-5.1232 E-02\end{array}$ \\
\hline $\begin{array}{l}3.4393 E-01 \\
3.5310 E-01 \\
3.6040 E-01 \\
5.1310 E-01 \\
5.1941 E-01\end{array}$ & $\begin{array}{l}8.4892 E-02 \\
8.6906 E-02 \\
8.8508 E-02 \\
4.8157 E-02 \\
4.8702 E-02\end{array}$ & $\begin{array}{l}1.8126 E-01 \\
1.8554 E-01 \\
1.8894 E-01 \\
4.8184 E-02 \\
4.8729 E-02\end{array}$ & $\begin{array}{l}-5.2802 E-02 \\
-5.4063 E-02 \\
-5.5066 E-02 \\
-1.4046 E-02 \\
-1.4207 E-02\end{array}$ \\
\hline $\begin{array}{l}5.1941 E-01 \\
5.1941 E-01 \\
5.1941 E-01 \\
5.0440 E-01 \\
4.7299 E-01\end{array}$ & $\begin{array}{l}4.8702 E-02 \\
4.8702 E-02 \\
4.8702 E-02 \\
5.3558 E-02 \\
6.3192 E-02\end{array}$ & $\begin{array}{l}4.8729 E-02 \\
4.8729 E-02 \\
4.8729 E-02 \\
6.5118 E-02 \\
9.7894 E-02\end{array}$ & $\begin{array}{l}-1.4207 E-02 \\
-1.4207 E-02 \\
-1.4207 E-02 \\
-1.8986 E-02 \\
-2.8543 E-02\end{array}$ \\
\hline $\begin{array}{l}.7299 E-01 \\
.7299 E-01 \\
.7905 E-01 \\
.6751 E-01\end{array}$ & $\begin{array}{l}6.3192 E-02 \\
6.3192 E-02 \\
6.1814 E-02 \\
3.4459 E-02\end{array}$ & $\begin{array}{l}9.7894 E-02 \\
9.7894 E-02 \\
9.2954 E-02\end{array}$ & $\begin{array}{l}-2.8543 E-02 \\
-2.8543 E-02 \\
-2.7103 E-02\end{array}$ \\
\hline
\end{tabular}


*Boron FLuORIDE $76 \mathrm{CM}$ HG 20 DFG C

\begin{tabular}{|c|c|c|c|c|c|}
\hline ATOM & FORMULA & MASS FRACPION & ATOM & FORMULA & MASS FRACTION \\
\hline B & $3.0000000 \mathrm{E}+00$ & $1.5958808 \mathrm{E}-01$ & $\mathrm{~F}$ & $6.0000000 E+00$ & $8.4041192 \mathrm{~F}-01$ \\
\hline & $\begin{array}{c}\text { FORMULA } \\
\text { F }\end{array}$ & $\begin{array}{c}\text { DENS ITY } \\
\text { RHO }(G / C C)\end{array}$ & $\begin{array}{l}\text { FUEL } \\
\text { RHOF }\end{array}$ & $\begin{array}{l}\text { DENSITY } \\
(G / C C)\end{array}$ & TEMPERATURE \\
\hline & & $2.8192308 E-03$ & 0. & & $16000 E \quad 02$ \\
\hline
\end{tabular}

PROBABILITY/PATH (/CM)

$\begin{array}{cc}\text { COLLIDE } & \text { MULT } \\ \text { SG(N) } & \text { NUSG } \\ 1.82 .26 E-04 & 0 . \\ 1.9927 E-04 & 0 . \\ 2.3722 E-04 & 0 . \\ 2.3469 E-04 & 0 . \\ 3.0225 E-04 & 0 . \\ 4.1953 E-04 & 0 . \\ 6.3660 E-04 & 0 . \\ 6.9997 E-04 & 0 . \\ 5.3587 E-04 & 0 . \\ 4.0989 E-04 & 0 . \\ 4.5587 E-04 & 0 . \\ 6.1818 E-04 & 0 . \\ 1.0687 E-03 & 0 . \\ 1.3575 E-03 & 0 . \\ 3.2194 E-03 & 0 . \\ & \\ 5.0806 E-03 & 0 . \\ 0.1467 E-03 & 0 . \\ 1.3401 E-02 & .0 \\ 1.7163 E-02 & 0 . \\ & \end{array}$

\begin{tabular}{|c|c|c|c|}
\hline \multicolumn{4}{|c|}{ SCATTER } \\
\hline $\operatorname{GSC}(\mathrm{N}, \mathrm{N})$ & $S G S I(N, N)$ & SGSO $(N, N+1)$ & $\operatorname{SGS} 1(N, N+1)$ \\
\hline $\begin{array}{l}1.2929 E-04 \\
1.4273 E-04 \\
1.7259 E-04 \\
2.0991 E-04 \\
2.2335 E-04\end{array}$ & $\begin{array}{l}2.4413 E-05 \\
2.6083 E-05 \\
2.9796 E-05 \\
3.4437 E-05 \\
3.6108 E-05\end{array}$ & $\begin{array}{l}2 E-05 \\
8 E-05 \\
2 E-05 \\
1 E-05 \\
7 E-05\end{array}$ & $\begin{array}{l}-1 \cdot 5 \\
-1 \cdot 6 \\
-1 \cdot 8 \\
-2 \cdot 1 \\
-2 \cdot 2\end{array}$ \\
\hline $\begin{array}{l}592 E-04 \\
762 E-04 \\
689 E-04 \\
236 E-04 \\
159 E-04\end{array}$ & $\begin{array}{l}4.7618 E-05 \\
6.8965 E-05 \\
7.5091 E-05 \\
3.0437 E-05 \\
2.2831 E-05\end{array}$ & $\begin{array}{l}E-05 \\
E-04 \\
E-04 \\
E-05 \\
E-05\end{array}$ & $\begin{array}{l}-3.0225 E-05 \\
-4.3926 E-05 \\
-4.7858 E-05 \\
-9.2538 E-06 \\
-6.9007 E-06\end{array}$ \\
\hline $\begin{array}{l}3.3733 E-04 \\
3.3377 E-04 \\
2.3377 E-04 \\
3.2648 E-04 \\
3.1189 E-04\end{array}$ & $\begin{array}{l}2.2061 E-05 \\
2.1868 E-05 \\
2.1868 E-05 \\
2.4069 E-05 \\
2.8471 E-05\end{array}$ & $\begin{array}{l}2 \cdot 2068 E-05 \\
2.1876 E-05 \\
2.187 \in E-05 \\
2.9167 E-05 \\
4.3751 E-05\end{array}$ & $\begin{array}{l}-5.6625 E-06 \\
-5.6029 E-06 \\
-6.6029 E-06 \\
-0.8038 E-06 \\
-1.3206 E-05\end{array}$ \\
\hline $\begin{array}{l}3.1527 E-C 4 \\
3.1864 E-04 \\
3.3394 E-C 4 \\
3.8758 E-04\end{array}$ & $\begin{array}{l}2.8723 E-05 \\
2.8976 E-05 \\
2.9215 E-05 \\
1.6396 E-05\end{array}$ & $\begin{array}{l}4.4136 E-05 \\
4.4522 E-05 \\
4.3596 E-05\end{array}$ & $\begin{array}{l}-1.3325 E-05 \\
-1.3444 E-05 \\
-1.3175 E-05\end{array}$ \\
\hline
\end{tabular}


114

*CAICIUM HYDRIDE.

*CAH

\begin{tabular}{|c|c|c|c|c|c|c|}
\hline ATOM & FORMULA & MASS FRACTION & ATOM & \multicolumn{2}{|c|}{ FORMULA } & MASS FRACTION \\
\hline $\mathrm{CA}$ & $5.4000000 \mathrm{E}+00$ & $9.5211000 \mathrm{E}-01$ & $\mathrm{H}$ & \multicolumn{2}{|c|}{$2.0400000 \mathrm{E}+00$} & $4.7890000 \mathrm{E}-\mathrm{O} 2$ \\
\hline & \multirow[t]{2}{*}{$\begin{array}{c}\text { FORMULA } \\
F\end{array}$} & $\begin{array}{c}\text { DENSITY } \\
\text { RHO }(G / C C)\end{array}$ & $\begin{array}{l}\text { FUEL } \\
\text { RHOF }\end{array}$ & $\begin{array}{l}\text { DENSITY } \\
(G / C C)\end{array}$ & \multicolumn{2}{|c|}{$\begin{array}{l}\text { TEMPERATURE } \\
T \text { (DEG K) }\end{array}$} \\
\hline & & $1.6999999 E \quad 00$ & 0. & & 2.9 & $6000 E \quad 02101$ \\
\hline
\end{tabular}

PROBABILITY/PATH (/CM)

\section{COLLIDE MULTIPLY $S G(N) \quad N U S G F(N)$}

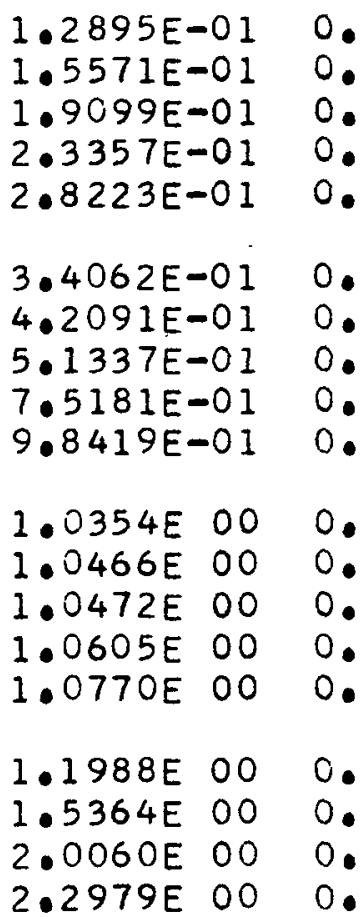

SCATTER

SGSO $(N, N)$ SGSI(N,N) SGSO $N, N+1) \quad S G S I(N, N+1)$

$7.7678 E-02$

$1.1034 E-01$

$1.2278 E-0 I$

$1.3988 E-01$

$1.5958 E-01$

4. $5652 E-01$

$5.8841 E-01$

$6.1742 E-01$

$6.2363 E-01$

$6.2363 E-01$

$5.4561 E-01$

4. $3732 E-01$

4. $8318 E-01$

$6.1751 E-01$

8.9021E-01

$2.2744 E \quad 00$
$1.4618 E-02$

$2.4562 E-02$

$3.2849 E-02$

$4 \cdot 3772 E-02$

$5.6956 E-02$

$7.2023 E-02$

$2.6882 E-02$

$3.5298 E-02$

$4.4916 E-02$

$5.6459 E-02$

$7.2330 E-02$

$9.0103 E-02$

$1.1496 \mathrm{E}-01$

$3.0688 E-01$

9.0605E-02

3.1107E-01

$2.5556 E-01$

$3.4242 E-01$

$1.1522 E-02$

$1.8146 E-02$

$2.6877 E-02$

$3.7415 E-02$

$4 \cdot 9458 E-02$

$6 \cdot 3910 E-02$

$8.3784 E-02$

$1.9403 E-01$

$1.3575 E-01$

$1.8241 E-01$

4.4021E-01 3.6152E-01

4. $4520 E-01 \quad 3.6561 E-01$

4.4498E-01 3.2867E-01

3. $9772 E-01 \quad 3.2500 E-01$

3.2226E-01 4.0327E-01

$1.9268 \mathrm{E}-01$

$1.9487 \mathrm{E}-01$

$1.8304 E-01$

$2.0213 E-01$

$2.6184 \mathrm{E}-01$

$3.5490 E-01$

4. $5308 E-01$

$2.7037 E-01$

4. $5184 E-01$

$5.9525 E-01$

$6.1755 E-01$

$1.0976 \mathrm{E} 00$

$3.0340 E-01$

$5.0473 E-02$ 
$\begin{array}{lll}0 & 1\end{array}$

1. $0509 E-02$

019

$1 \cdot 1498 E-03$

0115

$-5 \cdot 3366 E-08$

025

$9.4227 E-03$

O 211

5.1298E-05

0217

$\begin{array}{rrr}0 & & \\ 0 & 3 & \end{array}$

4.9447E-03

0314

$3.0947 \mathrm{E}-07$

046

$3.0157 E-02$

0412

$3.6677 E-05$

0418

0.511

5.8182E-04 7.8795E-05

0517

$2.4943 E-07-1.9954 E-07$

0611

$1.2270 E-03$

0
617

$6.3808 E-08$

O 712

$3.5594 E-04$

0718

$8.2950 E-08$

0814

$1.2284 E-05$

0911

$3.4342 \mathrm{E}-02$

0917

$1.9016 E-06$

01015

1.3775E-04

01114

$5.9194 E-03$

$0 \quad 1214$

4.4236E-02

01315

$5.9344 E-02$

$\begin{array}{lll}0 & 14 & 17\end{array}$

4. $3658 E-0$ ?

01618

$1.7050 E-01$
014

$6.3742 E-03$

0110

$1.5561 \mathrm{E}-04$

0116

0.26

$5.7152 E-C 3$

0212

$6.9620 E-06$

0218

0.

039

$6.5907 E-03$

0315

$5.6267 E-08$

04
$.8291 E-02$

0413

$5.0153 E-06$

0419

0.

$0 \quad 512$

0518

0612

$1.6603 E-04$

0618

0.

0713

4.8277E-05

0719

0 .

0815

2.0999E-06

0912

$4.6477 \mathrm{E}-03$

$0 ? 18$

$6.8782 E-07$

01016

$5.0752 E-05$

01115

$1.0747 \mathrm{E}-03$

01215

$8.0313 E-03$

01316

$2.1831 E-C 2$

01418

$1.6562 E-02$

01619

$8.5401 E-02$
015

$3.8662 E-03$

0111

2.1073E-05

0117

0 .

$\begin{array}{lll}0 & 2\end{array}$

$3.4664 \mathrm{E}-03$

0.213

$9.6640 E-07$

0219

0 .

0310

$8.9200 E-04$

0316

0 .

048

I. $1094 E-02$

0414

$5.3598 E-07$

057

3. $9296 E-02$

- 513

$1.0626 E-05$

0519

0 613

$2.2333 E-05$

O 619

0 .

0714

5. $7235 E-06$

O 810

$4.0553 E-02$

O 816

$7.3495 E-07$

0913

$6.2891 E-04$

0919

$3.2368 E-07$

$0 \quad 1017$

$1.8682 \mathrm{E}-05$

01116

$3.9523 E-04$

01216

$2.9546 E-03$

01317

$8.0313 E-03$

O 1419

$8.8463 E-03$

- 1719

$2.9042 E-01$
016

$2.3449 \mathrm{E}-03$

O 112

$2.8418 E-06$

O 118

0 .

028

$2.1025 E-03$

0214

$7.8889 E-08$

035

2. $2161 E-02$

0311

1.2071E-04

0317

0 .

049

$1.4787 \mathrm{E}-02$

0415

1.1485E-07

058

$2.3834 E-02$

C 514

$1.2970 E-06$

068

$5.0262 E-02$

0614

2. $8713 E-06$

079

$1.4359 E-01$

0715

$1.1613 E-06$

0 \& 11

$5.4682 E-03$

$0 \quad 817$

$3.1498 E-07$

0914

$7.65] .0 E-05$

$0 \quad 1012$

4. $5097 E-02$

01018

7.0059E-06

01117

$1.4545 E-04$

01217

$1.0869 E-03$

01318

$3.0467 E-03$

01517

$1.4702 E-01$ $\begin{array}{llllll}0 & 1 & 7 & 0 & 1 & 8\end{array}$

$1.4223 E-03 \quad 8.6265 E-04$

$\begin{array}{lll}0 & 1 & 13\end{array}$

$3.8691 E-07$

0119

0 .

029

$2.8024 E-03$

0215

$3.9445 E-08$

036

$1.3441 E-02$

0312

$1.6374 E-05$

0318

0.

0410

$2.0012 E-03$

0 4; 16

$1.9142 E-07-$

059

$3.1768 E-02$

0515

$2.4943 E-07$

069

$6.6993 E-02$

0615

$5 \cdot 1046 E-07$

0710

$1.9432 E-02$

O 716

4.1475E-07

$0 \& 12$

$7.4267 E-04 \quad 1.0064 E-04$

$\begin{array}{llllll}0 & 818 & 0 & 8 & 19\end{array}$

$5.2496 E-07-3.1498 E-07$

091509916

$1.3918 E-05 \quad 5.0979 E-06$

01013

$6.2385 E-03$

01019

$3.6930 E-06$

.01118

$5.5232 E-05$

01213

4.1220E-04

01319

$1.6272 E-03$

01518

01014

$7 \cdot 5845 E-04$

$0 \quad 1113$

4. $8681 E-02$

01119

2. $9423 E-05$

01219

2. $2025 E-04$

O 1416

$5.5773 E-02$

l. $1867 \mathrm{E}-01$

O 1519

2. $9571 E-02$ 
113

$6.5469 E-03$

119

$1.3670 E-04$

1115

$2.354 O E-07$

125

4. $5754 E-03$

1211

$2.4481 E-06$

1217

0. 38

$1.4602 E-03$

1314

$9.3855 E-08$

146

$1.8781 E-02$

1412

$1.5580 E-06$

1418

0. 511

$2.9135 E-05$

1517

$-1.3519 E-07$

1611

$7.3787 E-05$

$\begin{array}{lll}1 & 6 & 17\end{array}$

$4.2566 E-08$

1712

$1.4277 E-05$

1718

$2.2135 E-07$

1814

$1.0507 E-06$

1911

$6.8431 E-03$

1917

$3.5088 E-07$

11015

$5.4345 E-06$

11114

4. $7898 E-04$

11214

$9.2274 E-03$

11315

$1.6728 E-02$

11417

1. $0102 E-0 ?$ ?

11618

4. $0471 E-02$
114

$3.0954 E-03$

1110

$8.6815 E-06$

1116

0.

126

$2.1643 E-03$

1212

$6.0544 \mathrm{E}-07$

1218

0. 39

$1.2518 E-03$

1315

$3.7542 E-08$

147

$8.8765 E-U_{3}$

1413

$3.8311 E-07$

1419

0.512

$3.0117 E-06$

1518

$2.3503 E-07$

$1 \in 12$

$6.1294 E-06$

1618

$1.2770 E-07$

1713

$2.4072 E-06$

1719

1815

$2.8017 E-.07$

1912

$3.6115 E-04$

1918

$2.4292 E-07$

11016

$2.6448 E-06$

11115

$5.1251 E-05$

11215

$8.6480 E-04$

11316

$3.6920 E-03$

11418

$2.3054 \mathrm{~F}-03$

$116 \quad 19$

$-1.7345 E-02$
115

$1.4644 E-03$

1111

$1.1264 \mathrm{E}-06$

1117

0.

127

$1.0248 E-03$

1213

$1.8426 \mathrm{E}-07$

1219

0.

1310

$6.7275 E-05$

1316

$9.3855 E-08$

148

4. $1969 E-03$

1414

$1.7878 E-07$

157

$2.4471 E-02$

1513

$6.6557 E-07$

1519

0.

1613

$1.2770 E-06$

1619

0.

1714

5.5339E-07

1810

$9.8301 E-03$

1816

$1.4009 E-07$

1913

$2.5506 E-05$

1919

4. $5884 \mathrm{~F}-07$

$\begin{array}{lll}1 & 10 & 17\end{array}$

1.2680 E-06

11116

$1.5266 \mathrm{E}-05$

11215

$2.0345 E-04$

$\begin{array}{lll}1 & 13 & 17\end{array}$

$7.9920 E-04$

$\begin{array}{lll}1 & 14 & 19\end{array}$

$5.9603 E-74$

11719

$-5.0876 E-02$
116

$6.9344 E-04$

1112

$3.2055 E-07$

1118

0.

123

4. $8595 E-04$

1214

$5.2647 E-08$

135

$1.3803 E-02$

1311

5.1808E-06

1317

0.

149

$3.5842 E-03$

1415

$2.5540 E-08$

158

$1.1566 E-02$

1514

$1.9967 \mathrm{E}-07$

168

3.1300 E-02

1614

$2.5539 E-07$

179

$7.3322 E-02$

1715

3. $8737 E-07$

1811

$5.0932 E-04$

1817

$2.1013 E-07$

1914

$3.4683 E-06$

11012

$9.1866 E-03$

11018

$8.3328 E-07$

11117

5.7775E-06

1.1217

$5.1757 E-05$

11318

$1.6668 E-04$

$\begin{array}{lll}1 & 15 & 17\end{array}$

$5.5113 E-02$

$\begin{array}{llllll}1 & 1 & 7 & 1 & 1 & 8\end{array}$

3.2890E-04 1.564OE-04

111311114

$1.0685 E-07-1.4636 E-07$

1119124

0 .

129

4.1995E-04

1215

$9.2132 E-08$

136

$6.5244 \mathrm{E}-03$

1312

$9.6776 E-03$

1210

$2.4283 E-05$

1216

0.

137

$3.0855 E-03$

$9.1978 E-07$

1318

0.

1410

$1.8559 E-04$

1416

1313

2.8157E-07

1319

0.

1411

$1 \cdot 1902 E-05$

1417

$2.9691 E-07$

159

1510

$9.8604 E-03$

1515

$6.6557 E-08$

169

$2.6662 E-02$

1615

$1.7026 \mathrm{E}-07$

1710

$3.6842 E-03$

1716

$5.5339 E-08$

1812

$3.2710 E-05$

1818

$-4.7717 E-07$

1915

$9.9865 E-07$

$110 \quad 13$

4. $8524 E-04$

11019

1.4130 E-06

11118

$2.8313 E-06$

11218

$1.5807 E-05$

I 1319

$1.4430 E-05$

11518

$.0217 E-04$

1516

$6.6557 E-08$

1610

$1 \cdot 3458 E-03$

1616

4.2566E-08

1711

$1.9580 E-04$

1717

$5.5339 E-08$

1813

$4.3077 E-06$

1819

$7.5734 E-07$

1916

5.9379E-07

11014

$3.1085 E-05$ 
ATOM

FORMULA

EU $\quad 8.2400000 \mathrm{E}+00$
MASS FRACTION

8.6360000E-01
ATOM

0
FORMULA

$3.6000000 \mathrm{E}+00$
MASS FRACTION

1.3640000E-OI

\section{FORMULA \\ $F$

.

\author{
DENS ITY \\ RHO $(G / C C)$
}

$7.4199998 E \quad 00$

\author{
FUEL DENSITY \\ RHOF $(G / C C)$
}

\author{
TEMPERATURE \\ $T$ (DEG K)
}

PROBABILITY/PATH $(/ C M)$

\begin{tabular}{|c|c|}
\hline $\begin{array}{l}\text { COLLIDE } \\
\text { SG(N) }\end{array}$ & $\begin{array}{l}\text { MULTIPLY } \\
\text { NUSGF (N) }\end{array}$ \\
\hline $\begin{array}{l}2.7989 E-01 \\
3.1685 E-01 \\
3.1761 E-01 \\
3.2561 E-01 \\
4.0696 E-01\end{array}$ & $\begin{array}{l}0 \\
0 \\
0 \\
0 \\
0\end{array}$ \\
\hline $\begin{array}{l}4.3249 E-01 \\
4.0162 E-01 \\
3.9686 E-01 \\
4.6036 E-01 \\
5.8735 E-01\end{array}$ & $\begin{array}{l}0 . \\
0 \\
0 \\
0 . \\
0 .\end{array}$ \\
\hline $\begin{array}{ll}1.6668 E & 00 \\
5.2226 E & 00 \\
7.7625 E & 00 \\
7.7625 E & 00 \\
3.5891 E & 01\end{array}$ & $\begin{array}{l}0 . \\
0 . \\
0 . \\
0 . \\
0 .\end{array}$ \\
\hline 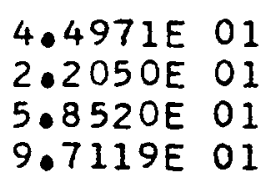 & $\begin{array}{l}0 . \\
0 . \\
0 . \\
0 .\end{array}$ \\
\hline
\end{tabular}

\begin{tabular}{|c|c|c|c|}
\hline \multicolumn{4}{|c|}{ SCATTER } \\
\hline $3 S O(N, N)$ & $S G S I(N \cdot N)$ & $\operatorname{SGSO}(N, N+1)$ & $\operatorname{SGS} 1(N, N+1)$ \\
\hline $\begin{array}{l}2.4222 E-01 \\
2.7025 E-01 \\
2.7083 E-01 \\
2.7689 E-01 \\
3.2621 E-01\end{array}$ & $\begin{array}{l}6.0177 E-03 \\
1.0293 E-02 \\
1.0381 E-02 \\
1.1306 E-02 \\
2.0547 E-02\end{array}$ & $\begin{array}{l}1.2271 E-02 \\
2.1205 E-02 \\
2.1389 E-02 \\
2.3324 E-02 \\
4.2653 E-02\end{array}$ & $\begin{array}{l}-3.9237 E-03 \\
-6.6465 E-03 \\
-6.7027 E-03 \\
-7.2921 E-03 \\
-1.3173 E-02\end{array}$ \\
\hline $\begin{array}{l}3.3320 E-01 \\
2.9743 E-01 \\
2.6909 E-01 \\
2.7302 E-01 \\
2.6040 E-01\end{array}$ & $\begin{array}{l}2.3404 E-02 \\
1.9595 E-02 \\
1.8708 E-02 \\
9.5547 E-03 \\
9.4709 E-03\end{array}$ & $\begin{array}{l}4.3491 E-02 \\
4.0696 E-02 \\
3.8877 E-02 \\
9.5524 E-03 \\
9.4690 E-03\end{array}$ & $\begin{array}{l}-1.5013 E-02 \\
-1.2556 E-02 \\
-1.1981 E-02 \\
-2.9393 E-03 \\
-2.9113 E-03\end{array}$ \\
\hline $\begin{array}{l}3.8656 E-01 \\
5.7580 E-01 \\
6.3887 E-01 \\
5.0900 E-01 \\
4.3892 E-01\end{array}$ & $\begin{array}{l}1.0310 E-02 \\
1.1583 E-02 \\
1.2030 E-02 \\
1.2347 E-02 \\
1.4060 E-02\end{array}$ & $\begin{array}{l}1.0303 E-02 \\
1.1555 E-02 \\
1.1973 E-02 \\
1.4851 E-02 \\
2.1442 E-02\end{array}$ & $\begin{array}{l}-3.1912 E-03 \\
-3.6265 E-03 \\
-3.7946 E-03 \\
-4.6701 E-03 \\
-6.6623 E-03\end{array}$ \\
\hline $\begin{array}{l}.0158 E-C I \\
.3975 E-01 \\
.7430 E-01 \\
.2680 E-01\end{array}$ & $\begin{array}{l}1.4619 E-02 \\
1.4135 E-02 \\
1.3705 E-02 \\
7.8408 E-03\end{array}$ & $\begin{array}{l}2.2276 E-02 \\
2.1557 E-02 \\
2.0332 E-02\end{array}$ & $\begin{array}{l}-6.9421 E-03 \\
-6.6973 E-03 \\
-6.2916 E-03\end{array}$ \\
\hline
\end{tabular}




\begin{tabular}{|c|c|c|c|c|c|c|}
\hline ATOM & FORMULA & MASS FRACTION & ATOM & \multicolumn{2}{|c|}{ FORMIJA } & MASS FRACTION \\
\hline $\begin{array}{l}\mathrm{FE} \\
\mathrm{AL}\end{array}$ & $\begin{array}{l}4.0000000 \mathrm{E}+00 \\
5.1000000 \mathrm{E}+00\end{array}$ & $\begin{array}{l}6.5000000 \mathrm{E}-\mathrm{O} 1 \\
5.0000000 \mathrm{E}-\mathrm{O} 2\end{array}$ & $\begin{array}{l}\mathrm{CP} \\
\mathrm{NB}\end{array}$ & \multicolumn{2}{|c|}{$\begin{array}{l}4.2000000 E+00 \\
5.3600000 E+00\end{array}$} & $\begin{array}{l}2.5000000 \mathrm{E}-01 \\
3.0000000 \mathrm{E}-02\end{array}$ \\
\hline $\mathrm{Y}$ & $5.2440000 \mathrm{E}+00$ & $2.0000000 \mathrm{E}-02$ & & & & \\
\hline & \multirow{3}{*}{$\begin{array}{c}\text { FORMULA } \\
\text { F }\end{array}$} & DENS ITY & FUEL & \multirow{2}{*}{$\begin{array}{l}\text { DENSITY } \\
(G / C C)\end{array}$} & \multicolumn{2}{|c|}{ TEMPERATURE } \\
\hline & & RHO $\quad(G / C C)$ & RHOF & & \multicolumn{2}{|c|}{$T(D E G \quad K)$} \\
\hline & & $7.2770012 E \quad 00$ & 0. & & $2 \cdot 5$ & $6000 E \quad 02101$ \\
\hline
\end{tabular}

PROBABILITY/PATH (/CM)

\begin{tabular}{|c|c|c|c|c|c|}
\hline COLLIDE & MUL T I PLY & & & E & \\
\hline & $\operatorname{NUSGF}(N)$ & $\operatorname{SGSO}(N, N)$ & $\operatorname{SGSI}(N, N)$ & SGSO $(N, N+1)$ & $\operatorname{SGSI}(N, N+1)$ \\
\hline $\begin{array}{l}1.8684 E-01 \\
2.0439 E-01 \\
2.2245 E-01 \\
2.1273 E-01 \\
1.9154 E-01\end{array}$ & $\begin{array}{l}0 . \\
0 . \\
0 \\
0 \\
0\end{array}$ & $\begin{array}{l}1.6327 E-01 \\
1.7781 E-01 \\
1.9042 E-01 \\
1.8204 E-01 \\
1.6445 E-01\end{array}$ & $\begin{array}{l}9.4484 E-03 \\
1.0178 E-02 \\
1.1478 E-02 \\
1.1055 E-0 ? \\
1.0643 E-02\end{array}$ & $\begin{array}{l}1.8543 E-02 \\
1.9526 E-02 \\
2.1409 E-02 \\
2.0739 E-02 \\
2.1023 E-02\end{array}$ & $\begin{array}{r}-1.6344 E-03 \\
-0.4396 E-04 \\
5.5140 E-04 \\
4.0329 E-04 \\
-1.2268 E-03\end{array}$ \\
\hline $\begin{array}{l}2.3483 E-01 \\
2.7054 E-01 \\
2.8669 E-01 \\
3.3328 E-01 \\
4.7214 E-01\end{array}$ & $\begin{array}{l}0 . \\
0 . \\
0 . \\
0 . \\
0 .\end{array}$ & $\begin{array}{l}1.9438 E-01 \\
2.3109 E-01 \\
2.6346 E-01 \\
3.2638 E-01 \\
4.6259 E-01\end{array}$ & $\begin{array}{l}1.4005 E-02 \\
1.4586 E-02 \\
1.1365 E-02 \\
6.5868 E-03 \\
8.6928 E-03\end{array}$ & $\begin{array}{l}2.6131 E-02 \\
2.7816 E-02 \\
2.3076 E-02 \\
6.5846 E-03 \\
8.6902 E-03\end{array}$ & $\begin{array}{r}2.3007 E-03 \\
5.1571 E-05 \\
-7.4603 E-03 \\
-2.1320 E-03 \\
-2.8246 E-03\end{array}$ \\
\hline $\begin{array}{l}6.0775 E-01 \\
6.5516 E-01 \\
6.8630 E-01 \\
5.8988 E-01 \\
7.0046 E-01\end{array}$ & $\begin{array}{l}0 . \\
0 . \\
0 . \\
0 .\end{array}$ & $\begin{array}{l}5.9434 E-01 \\
6.3898 E-01 \\
6.6598 E-01 \\
6.5812 E-01 \\
6.4664 E-01\end{array}$ & $\begin{array}{l}1.1093 E-02 \\
1.1800 E-02 \\
1.2277 E-02 \\
1.3544 E-02 \\
1.6121 E-02\end{array}$ & $\begin{array}{l}1.1089 E-02 \\
1.1795 E-02 \\
1.2274 E-02 \\
1.6285 E-02 \\
2.4324 E-02\end{array}$ & $\begin{array}{l}-3.6066 E-03 \\
-3.8383 E-03 \\
-3.9917 E-03 \\
-5.2997 E-03 \\
-7.9110 E-03\end{array}$ \\
\hline $\begin{array}{l}7.1211 \mathrm{E}-01 \\
7.3731 \mathrm{E}-01 \\
7.9184 \mathrm{E}-01 \\
8.2954 \mathrm{E}-01\end{array}$ & $\begin{array}{l}0 . \\
0 . \\
0 . \\
0 .\end{array}$ & $\begin{array}{l}6.3942 E-01 \\
6.3331 E-01 \\
6.3509 E-O I \\
6.5676 E-01\end{array}$ & $\begin{array}{l}1.5950 E-02 \\
1.5800 E-02 \\
1.5409 E-02 \\
8.0527 E-03\end{array}$ & $\begin{array}{l}2.4065 E-02 \\
2.3838 E-02 \\
2.2615 E-02\end{array}$ & $\begin{array}{l}-7.8280 E-03 \\
-7.7548 E-03 \\
-7.3563 E-03\end{array}$ \\
\hline
\end{tabular}

RELIABILITY LIMITATION: FIRST-SIGNIFICANT FIGURE UNCERTAINTY IN HIGH ENERGY SCATTER CROSS SECTIONS OF ALUMINUM CONTENT. 
$\begin{array}{lll}0 & 1 & 3\end{array}$ $3.8793 E-03$

019

0

0115

025

$2.3218 E-03$

0211

0

0.

038

$9.9187 E-04$

0314

0.46

$4.4759 E-03$

0412

0.418

$\begin{array}{rrr}0 . & & \\ 0 & 511\end{array}$

0.517

$\begin{array}{rrr}0 . & & \\ 0 & 6 & 11\end{array}$

$\begin{array}{rrr}0 & & \\ 0 & 6 & 17\end{array}$

0.712

0.
0

0

O 814

0.911

$\begin{array}{rrr}0 . & & \\ 0 & 9 & 17\end{array}$

0.1015

0.

$\begin{array}{rrr}0 . & 11 & 14 \\ 0 & 1214\end{array}$

$\begin{array}{rrr}0 . & & \\ 0 & 1315\end{array}$

$\begin{array}{rrr}0 . & & \\ 0 & 14 & 17\end{array}$

$\begin{array}{rll}0 . & & \\ 0 & 16 \quad 18\end{array}$

0 .
014 0110

0.116

026

$8.6707 E-04$

0
0

$\begin{array}{rrr}0 . & & \\ 0 & 218\end{array}$

0 .

- 039

$8.1213 E-04$

0315

0.47

$2.7148 E-03$

0413

0.413

0 .

$0 \quad 512$

0.
0

$\begin{array}{rrr}0 . & & \\ 0 & 6 & 12\end{array}$

0.618

0.713

0.719

0

0815

$\begin{array}{rrr}0 . & & \\ 0 & 12\end{array}$

$\begin{array}{rrr}0 . & & \\ 0 & 918\end{array}$

0.1016

0 .

$\begin{array}{lll}0 & 11 & 15\end{array}$

$\begin{array}{rrr}0 . & \\ 0 & 1215\end{array}$

0.

01316

0.1418

$\begin{array}{rrr}0 . & & \\ 0 & 16 \quad 19\end{array}$

$c$.
01

0111

0
0
0
0

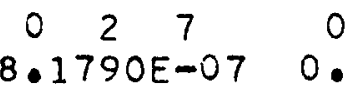

० 213

0.

0 .

0310

0 .

C 315

$\begin{array}{rrr}0 . & & \\ 0 & 4 & 8\end{array}$

$1.6466 E-03$

0414

0.

057

5.2399E-03

O 513

0 .

O 519

0.

0.619

0.714

0.
0

0 .

$0 \quad 816$

0.013

$\begin{array}{ll}0.0 & 9 \\ 0 & 19\end{array}$

0.1017

$0 .: 1116$

0.01216

$\begin{array}{lll}0 & 13 & 17\end{array}$

0.01419

0.01719

0 .
0

O 35

$4.4453 E-03$

0311

0 .

0317

0.49

$1.0487 E-03$

0415

0.

058

$7.5061 E-04$

0514

$\begin{array}{rrr}0 & & \\ 0 & 6 & 8\end{array}$

$7.4405 E-03$

0614

0.079

1.1507E-02

O 715

0 .

$\begin{array}{lll}0 & 8 & 11\end{array}$

0.

$\begin{array}{lll}0 & 17\end{array}$

$0: 914$

0 .

01012

0.01018

0 .

$\begin{array}{lll}0 & 11 & 17\end{array}$

0 .

$0 \quad 1217$

0 .

$\begin{array}{lll}0 & 13 & 18\end{array}$

0.

01517

0 . $\begin{array}{rrrrrr}0 & 1 & 7 & 0 & 1 & 8 \\ 0 . & & & 0 . & \\ 0 & 1 & 13 & 0 & 1 & 14 \\ 0 & & & 0 . & & \\ 0 & 1 & 19 & 0 & 2 & 4\end{array}$

0 . $\quad 3.8280 E-03$

$\begin{array}{lllll}0 & 2 & 0 & 210\end{array}$

0.

0

215

0.016

0

$2.6962 E-03$

037

$1.6353 E-03$

03120313

0.

0318

0

0319

0 .

0.416

0

$0 \quad 515$

069

$6.7880 E-03$

0615

0.

0

710

$\begin{array}{lll}0 & 716\end{array}$

0 .

$0 \quad 812$

0411

0417

0.

0510

0.

0516

0610

0.

0616

0711

0

0717

0.

0813

0.

$\begin{array}{lll}0 & 818\end{array}$

$\begin{array}{lll}0.0 & 8 & 19\end{array}$

0.015

0.0916

0 .

01013

0.

01019

01014

0.

01113

0 .

01118

01119

0 .

01218

0 .

$0 \quad 1219$

0.

01319

0.01518

0.

01416

0.

01519 


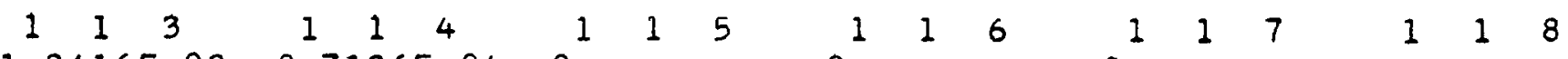

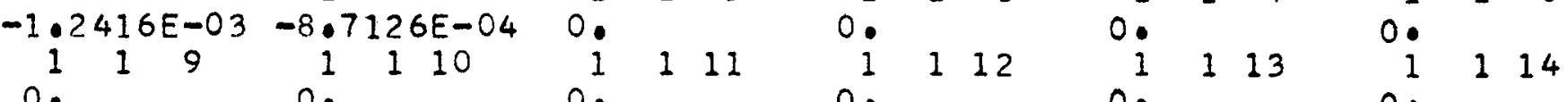
0. $\begin{array}{lllllll}1 & 1 & 15 & 1 & 1 & 16 & 0\end{array}$ 115 $\begin{array}{llllllllll}1 & 2 & 5 & 1 & 2 & 6 & 1 & 2 & 7\end{array}$ $-1.0065 E-03-6.9677 E-04-8.1461 E-07 \quad 0$ 1212 1213 0. 1 $217 \quad 0$. 1 8 1 1218 1219 i 310 139 146 $1.2928 E-03$

$$
0
$$$$
0
$$

0

0

$\begin{array}{lll}0 & 6 & 11\end{array}$

$0: 617 \quad 0 ; \quad 18$

$0: 712 \quad 0: 713$

00

0.814

$\begin{array}{lll}0.9 & 14 \\ 0 & 9 & 11\end{array}$

$\begin{array}{lll}0.0 & 9 & 17\end{array}$

$\begin{array}{ccc}0 & 10 \quad 15\end{array}$

i 1114

$0 \begin{array}{lll}1 & 11 & 14 \\ 1 & 12 & 14\end{array}$

0.1315

0.

$\begin{array}{lll}1 & 14 & 17\end{array}$

$\begin{array}{lll}0 & 16 \quad 18\end{array}$

11618
1315

147

$-1.3147 E-04$

1413

i 419

i 512

0.

1518

0.

0.

1. 316

148

1414

$\begin{array}{lll}0 & 1 & \\ 1 & 5\end{array}$ $-2.3237 E-0$

$\begin{array}{lll}1 & 5 & 13 \\ 0 & & \end{array}$

$\begin{array}{lll}1 & 5 & 19\end{array}$

1613

$\begin{array}{lll}0.1 & 6 & 19\end{array}$

$\begin{array}{lll}0.14 & \end{array}$

0.1810

1719

0.

1815

$\begin{array}{lll}0 & 9 & 12\end{array}$

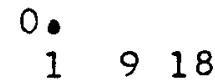

0.

0.

o. 1215

0 .

11316

0.

$\begin{array}{lll}1 & 14 & 18\end{array}$

i 1619

$$
0 \text {. }
$$

0.810

$\begin{array}{lll}1 & 8 & 16\end{array}$

1913

0.19
$1 \quad 9 \quad 19$

i 1017

0.1116

0.1116

c. 1317

$\begin{array}{lll}1 & 13 & 17 \\ 0 . & & \end{array}$

11419

$\begin{array}{lll}01719 & \end{array}$
$-6.4007 E-04$

11016

11115

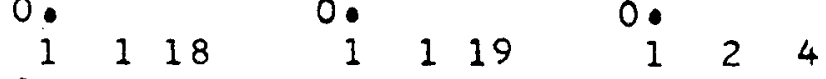

$0.10 .8332 E-04$

$\begin{array}{lllllllll}1 & 2 & 8 & 1 & 2 & 9 & 1 & 2 & 10\end{array}$

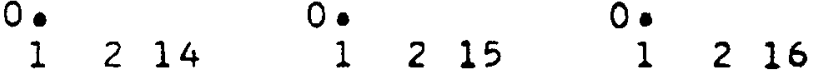

$\begin{array}{lllllllll}0 & 1 & 3 & 0 & 0 & 0 & & & \\ 1 & 5 & 1 & 3 & 1 & 3\end{array}$

$1.8295 E-03 \quad 3.9833 E-04-1.7466 E-04$

$\begin{array}{lllllllll}1 & 3 & 11 & 1 & 3 & 12 & 1 & 313\end{array}$

0.000

$\begin{array}{lllllllll}1 & 3 & 17 & 1 & 3 & 18 & 1 & 3 & 19\end{array}$

0.0 .00

$\begin{array}{lllllllll}1 & 4 & 9 & 1 & 4 & 10 & 1 & 4 & 11\end{array}$

$-7.8857 E-04 \quad 0$.

1415

0.

$\begin{array}{lllll}4 & 16 & 0 & 1 & 4\end{array}$

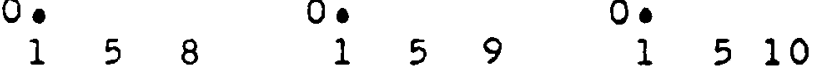

$\begin{array}{ll}-6.3048 E-04 & 0 \\ 0.14 & \end{array}$

$\begin{array}{llll}5 & 15 & 1 & 5^{\circ} 16\end{array}$

0 .

$\begin{array}{llllllllll}1 & 6 & 8 & 1 & 6 & 9 & 1 & 6 & 10\end{array}$

$7.3062 E-04-3.3236 E-03$

0

$1 \quad 6 \quad 14$

1616

0.0 .

179

$-3.0857 E-03$

1715

$\begin{array}{lll}1 & 7 & 10\end{array}$

17.11

i 716

1811

0

1813

0.

1

$\begin{array}{lll}1 & 8 & 17\end{array}$

0.914

0.

11012

i 1018

0.

1915

0 .

1916

$\begin{array}{lll}1 & 9 & 15 \\ 1 & 10 & 13\end{array}$

0.

0.1117

c. 1117

11019

0.1118

0.1118

0.

11014

$\begin{array}{lll}1 & 1217 \\ 0.1 & & 17\end{array}$

$\begin{array}{lll}1 & 12 & 18\end{array}$

I 1113

11318

11319

$\begin{array}{lll}0 & 1517\end{array}$

0.

11518

I 1119

11219

0.

11416

0 .

0 .
1615

$\begin{array}{lll}1 & 7 & 17\end{array}$

1818

1819 
*FECRAL

*FECRAL

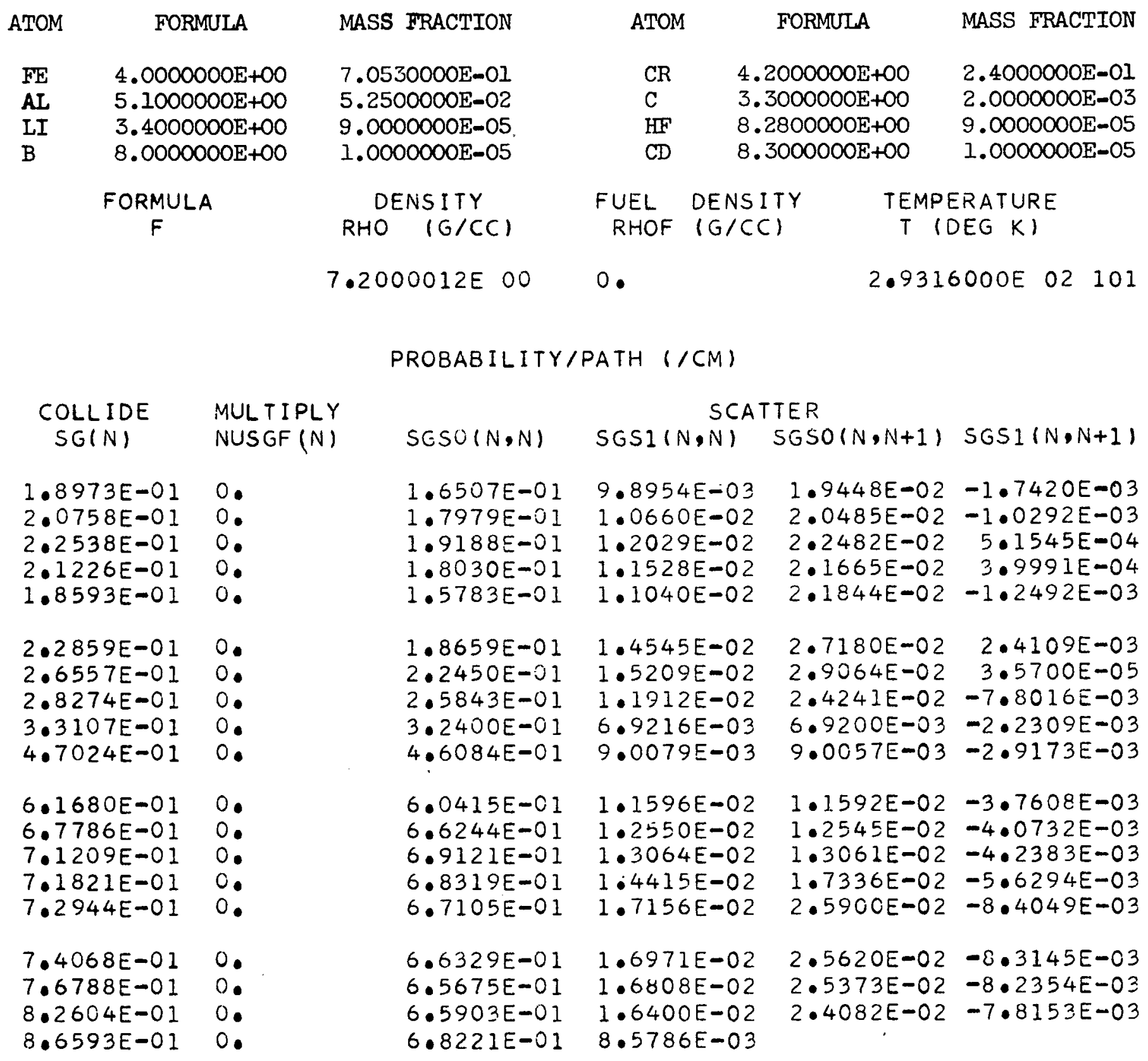

RELIABIIITY LIMITATION: FIRST-SIGNIFICANT-FIGURE UNCERTAINTY IN HIGH ENERGY SCATIEER CROSS SECTIONS OF ALUMINUM CONIENT. 


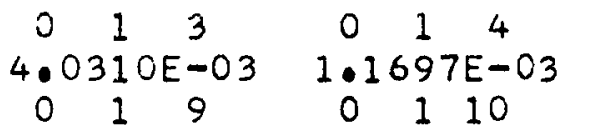

0 .

0115

0.25

$2.4121 E-03$

0

0

0

$\begin{array}{ll}0 & 3\end{array}$

$1.0304 E-03$

0314

0.46

4.650OE-03

0

0

$\begin{array}{rrr}0 . & & \\ 0 & 517\end{array}$

0.611

$\begin{array}{rrr}0 . & & \\ 0 & 17\end{array}$

0.
0

0.718

0 .

$\begin{array}{lll}0 & 8 & 14\end{array}$

0.
0

$\begin{array}{rrr}0 . & & \\ 0 & 9 & 17\end{array}$

0.1015

$\begin{array}{rll}0 . & & \\ 0 & 11 & 14\end{array}$

0.1214

$\begin{array}{rll}0 . & & \\ 0 & 1315\end{array}$

$\begin{array}{rrr}0 . & & \\ 0 & 14 & 17\end{array}$

$\begin{array}{rll}0 . & & \\ 0 & 1618\end{array}$

0 .
0

026

$9.0079 E-04$

0212

0.

0218

0.

039

$8.4371 E-04$

0315

0

047

$2.8204 E-03$

0413

0.
0

0.

$0 \quad 512$

0.

0518

0.612

0.618

$\begin{array}{rrr}0 . & & \\ 0 & 7 & 13\end{array}$

0 .

0719

0.

0815

$\begin{array}{rrr}0 . & & \\ 0 & 9 & 12\end{array}$

0.
0

0.51016

0.1115

0.1215

0 .

01316

0.1418

$\begin{array}{rrr}0 . & 1619\end{array}$

0 .
- 116
0

0

0117

0.

- 27

$8.4970 E-07$

0213

0.

0

0

219

310

0 .

o 316

0 .

C 48

$1.7106 E-03$

0414

0.

0 57

5.4457E-03

0513

0 .

0519

0.613

0.
0

$\begin{array}{rrr}0 . & & \\ 0 & 7 & 14\end{array}$

$\begin{array}{rrr}0 . & & \\ 0 & 810\end{array}$

0 .

- 816

0.913

0.
0

$\begin{array}{rrr}0 . & & \\ 0 & 10 \quad 17\end{array}$

$\begin{array}{rrr}0 . & & \\ 0 & 11 & 16\end{array}$

0.1616

0 .

$\begin{array}{lll}0 & 13 & 17\end{array}$

0.1419

0 .

01719

0.

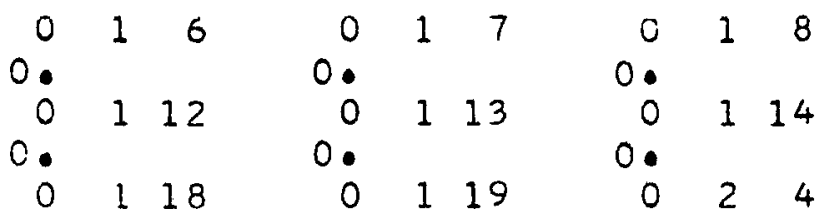

$0 . \quad 0 . \quad 3.9775 E-03$

$\begin{array}{lllllllll}0 & 2 & 8 & 0 & 2 & 9 & 0 & 2 & 10\end{array}$

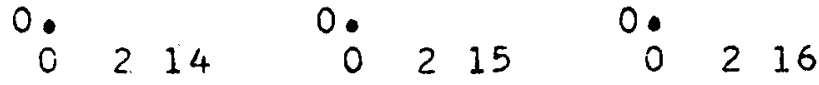

$0.0 \begin{array}{lllllll}0 & 0 . & 0 . & 0 & \\ 0 & 3 & 0 & 3 & 6 & 0 & 3\end{array}$

4.6197E-03

0,311

$2.8010 E-03$

$1.6989 E-03$

0 . 0.

$\begin{array}{llllllll}0 & 3 & 17 & 0 & 3 & 18 & 0 & 319\end{array}$

0.0 .

0490

$1.0894 E-03 \quad 0$.

C 415

0.

0050

0

$7.7980 E-04 \quad 0$.

$0514 \quad 0$

$0 \quad 515$

0 .

0 .

410

0.

0411

068

069

$7 \cdot 7330 E-03$

0614

0.37

0615

0.

1.1957E-02

0

416

0.

417

0715

0.

710

0 . 0 .

0811

0.

0 .

0817

0.

$0 \quad 818$

0 .

0914

0.

0915

0 .

0.

$0 \quad 1012$

01013

0.1018

0 .

01019

0.1117

0 .

01118

0.01217

0 .

01218

0 .

01318

0 .

01319

0 .

0 1517

0.

01518

0 .

0510

0.

0516

0 .

0610

0 .

0616

0.

0711

$\begin{array}{rrr}0 . & & \\ 0 & 7 & 17\end{array}$

0 .

0813

0 .

0819

0.

0916

0.

01014

0.

01113

0 .

01119

0.

$\begin{array}{lll}0 & 1219\end{array}$

0 .

01416

0 .

$0 \quad 1519$ 
$\begin{array}{llllllllllllllllll}1 & 1 & 3 & 1 & 1 & 4 & 1 & 1 & 5 & 1 & 1 & 6 & 1 & 1 & 7 & 1 & 1 & 8\end{array}$

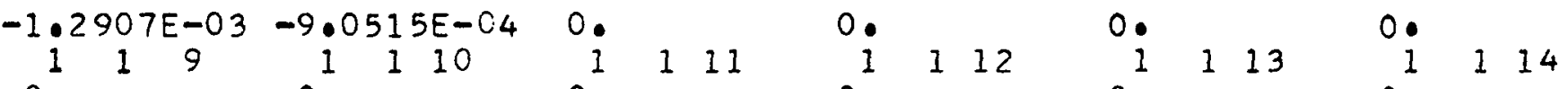

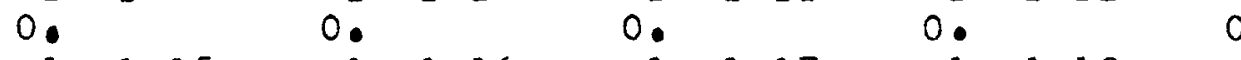
$\begin{array}{llllllllllll}1 & 1 & 15 & 1 & 1 & 16 & 1 & 1 & 17 & 1 & 1 & 18\end{array}$

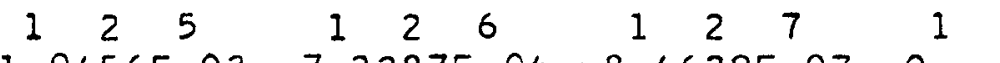

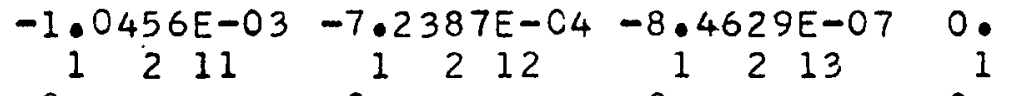
0.00 .000

1

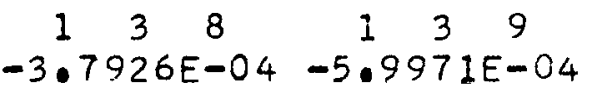
13.14 1315

0.

146 $1.3430 E-03-1$ 1412 0. 0

0.

0. 517

$\begin{array}{rrr}0 . & & \\ 1 & 6 & 11\end{array}$

0.

1

0

712

1718

0.
1

0 .

1911

0

1

0.11015

0.

11114

0. 1214

0 .

11315

0

$114 \quad 17$

0.

11618
0.

147

$-1.3658 E-04$

1413

O. 419

0

1512

0. 518

0.

1612

$\begin{array}{lll}1 & 618\end{array}$

0

1713

0. 719

0.

1815

0
1

0

1 918

0.

$110 \quad 16$

0.

] $11 \quad 15$

0.

11215

11316

0.1418

$\begin{array}{lll}1 & 1418\end{array}$

$116 \quad 19$
1219

1310

0 .

1316

0.

148

$-6.6496 E-04-8.1924 E-04$

1414

0.57

157

$-2.4157 E-0$
1513

0. 519

0.13

0.1619

$\begin{array}{ccc}1 & 619 \\ 1 & 714\end{array}$

0. 810

0.

1816

1913

0.919

0.

$\begin{array}{lll}1 & 10 & 17\end{array}$

0.1116

0. 1216

$\begin{array}{lll}1 & 1317\end{array}$

0.1317

11419

1 1719
135

$1.8993 E-03$

1311

0.

$\begin{array}{lll}1 & 3 & 17\end{array}$

0 .

1415

0.

158

$-6.5499 E-04$

1514

0.

168

$7.5656 E-04$

1614

i 79

$-3.2077 E-03$

1715

0 .

$\begin{array}{lll}1 & 8 & 11 \\ 0 . & & \end{array}$

1817

i 914

0 .

11012

0.

11018

$\begin{array}{lll}0.1117 & 11\end{array}$

0.

11217

1 1318

0.1517

11517
$0 \cdot 119$

124

$0.1 .8986 E-04$

1291210

1. $215 \quad 0.12216$

0.0 .

$\begin{array}{llllll}1 & 3 & 6 & 1 & 3 & 7\end{array}$

4.1382E-04 -1.8145E-04

1. $312 \quad 1313$

0 .

$\begin{array}{lllll}1 & 3 & 18 & 1 & 3.19\end{array}$

$0 . \quad 0$.

$1410 \quad 1$

$1416 \quad 1417$

0.00

159

1510

0.

1515

0.

1516

0.0.

1691610

$3.4528 \mathrm{E}-03 \quad 0$.

1616

0

$\begin{array}{lll}1 & 7 & 17\end{array}$

1716

0.

1812

0

$\begin{array}{lll}1 & 8 & 13\end{array}$

1
1

0.

0.

11013

0.

11019

0.

11118

$0 \cdot 1218$

11218

11319

0.

0
1916

0.

$110 \quad 14$

0.

11113

0.

11119

0 .

11219

0 .

11416

0 .

11519
1819 $\begin{array}{ccc}0 . & & \\ 1 & 7 & 11\end{array}$ 


\begin{tabular}{|c|c|c|c|c|c|c|}
\hline \multirow{2}{*}{$\begin{array}{l}\text { ATOM } \\
\text { GD }\end{array}$} & \multirow{2}{*}{$\begin{array}{c}\text { FORMULA } \\
8.2040000 \mathrm{E}+00\end{array}$} & \multirow{2}{*}{$\begin{array}{l}\text { MASS FRACTION } \\
8.6730000 E-01\end{array}$} & ATOM & \multicolumn{2}{|c|}{ FORMULA } & MASS FRACTION \\
\hline & & & 0 & 3.600 & $E+\infty$ & $1.3270000 \mathrm{E}-01$ \\
\hline & \multirow[t]{2}{*}{$\begin{array}{c}\text { FORMULA } \\
F\end{array}$} & $\begin{array}{c}\text { DENS ITY } \\
\text { RHO }(G / C C)\end{array}$ & $\begin{array}{l}\text { FUEL } \\
\text { RHOF }\end{array}$ & $\begin{array}{l}\text { DENSITY } \\
(G / C C)\end{array}$ & \multicolumn{2}{|c|}{$\begin{array}{l}\text { TEMPERATURE } \\
T(D E G \quad K)\end{array}$} \\
\hline & & $7.4100021 E 00$ & 0. & & $2 \cdot 93$ & $6000 E \quad 02$ \\
\hline
\end{tabular}

PROBABILITY/PATH (/CM)

\begin{tabular}{|c|c|c|c|c|c|}
\hline COLL IDE & MULT IPLY & & & $E R$ & \\
\hline & NUSGF $(N)$ & $S G S O(N, N)$ & $\operatorname{SGSI}(N, N)$ & SGSO $(N \cdot N+1)$ & $\operatorname{SGSI}(N, N+1)$ \\
\hline $\begin{array}{l}2.2364 E-01 \\
2.4750 E-01 \\
2.2394 E-01 \\
2.0753 E-01 \\
2.7485 E-01\end{array}$ & $\begin{array}{l}0 . \\
0 . \\
0 . \\
0 . \\
0 .\end{array}$ & $\begin{array}{l}2.1143 E-01 \\
2.2664 E-01 \\
2.0316 E-01 \\
1.8501 E-01 \\
2.3291 E-01\end{array}$ & $\begin{array}{l}5.4745 E-03 \\
9.4720 E-03 \\
9.2465 E-03 \\
9.8004 E-03 \\
1.8789 E-02\end{array}$ & $\begin{array}{l}1.1157 E-02 \\
1.9520 E-02 \\
1.9065 E-02 \\
2.0309 E-02 \\
3.9097 E-02\end{array}$ & $\begin{array}{l}-3.5684 E-03 \\
-6.1109 E-03 \\
-5.9604 E-03 \\
-6.2940 E-03 \\
-1.2016 E-02\end{array}$ \\
\hline $\begin{array}{l}3.0046 E-01 \\
2.7152 E-01 \\
2.6822 E-01 \\
2.7481 E-01 \\
2.9640 E-01\end{array}$ & $\begin{array}{l}0 . \\
0 . \\
0 . \\
0 . \\
0 .\end{array}$ & $\begin{array}{l}2.5172 E-01 \\
2.2898 E-01 \\
2.2547 E-01 \\
2.5302 E-01 \\
2.5302 E-01\end{array}$ & $\begin{array}{l}2.1658 E-02 \\
1.8190 E-02 \\
1.7654 E-02 \\
9.1841 E-03 \\
9.1841 E-03\end{array}$ & $\begin{array}{l}2 E-02 \\
+4 E-02 \\
26 E-02 \\
14 E-03 \\
4 E-03\end{array}$ & $\begin{array}{l}-1.3844 E-02 \\
-1.1635 E-02 \\
-1.1294 E-02 \\
-2.8234 E-03 \\
-2.8234 E-03\end{array}$ \\
\hline $\begin{array}{l}3.5511 E-01 \\
5.7690 E-01 \\
1.3110 E 00 \\
3.5320 E 00 \\
3.2852 E 00\end{array}$ & $\begin{array}{l}0 . \\
0 . \\
0 . \\
0 . \\
0 .\end{array}$ & $\begin{array}{l}2.5302 E-01 \\
3.1432 E-01 \\
4.9821 E-01 \\
1.0451 E 00 \\
9.1375 E-01\end{array}$ & $\begin{array}{l}9.1841 E-03 \\
9.5820 E-03 \\
1.0777 E-02 \\
1.5905 E-02 \\
1.7864 E-02\end{array}$ & $\begin{array}{l}9.1814 E-03 \\
9.5776 E-03 \\
1.0766 E-02 \\
1.9110 E-02 \\
2.7080 E-02\end{array}$ & $\begin{array}{l}-2.8234 E-03 \\
-2.9560 E-03 \\
-3.3554 E-03 \\
-6.0954 E-03 \\
-8.5856 E-03\end{array}$ \\
\hline $\begin{array}{ll}1.3095 E & 01 \\
1.5437 E & 02 \\
6.3236 E & 02 \\
8.9095 E & 02\end{array}$ & $\begin{array}{l}0 . \\
0 . \\
0 . \\
0 .\end{array}$ & 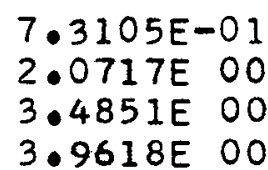 & $\begin{array}{l}1.6299 E-02 \\
2.8042 E-02 \\
3.9812 E-02 \\
2.2845 E-02\end{array}$ & $\begin{array}{l}2.4703 E-02 \\
4.2249 E-02 \\
5.8158 E-02\end{array}$ & $\begin{array}{l}-7.8160 E-03 \\
-1.3684 E-02 \\
-1.9046 E-02\end{array}$ \\
\hline
\end{tabular}




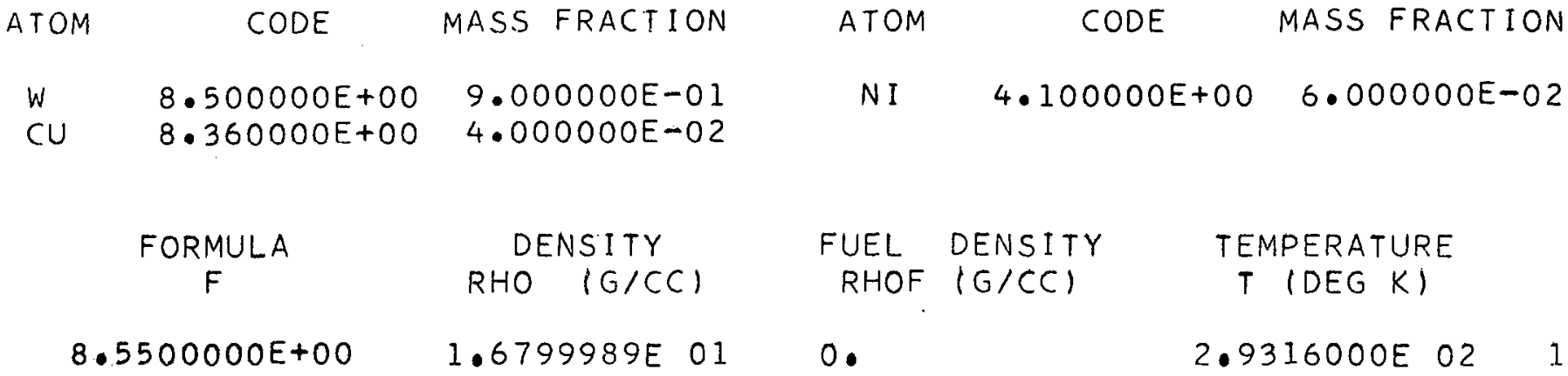

PROBABILITY/PATH (/CM)

$\begin{array}{cl}\text { COLLIDE } & \text { MULTIPLY } \\ \text { SGIN) } & \text { NUSGF(N) } \\ 3.0266 E-01 & 0 . \\ 3.4234 E-01 & 0 . \\ 3.7983 E-01 & 0 . \\ 3.9341 E-01 & 0 . \\ 3.9722 E-01 & 0 . \\ & \\ 4.1482 E-01 & 0 . \\ 4.5143 E-01 & 0 . \\ 4.9346 E-01 & 0 . \\ 5.9195 E-01 & 0 . \\ 8.1376 E-01 & 0 . \\ 1.0014 E-00 & 0 . \\ 1.1514 E \text { 0 } & 0 . \\ 1.8947 E-00 & 0 . \\ 1.5930 E-00 & 0 . \\ 6.7027 E-01 & 0 . \\ 7.4985 E-01 & 0 . \\ 9.0351 E-01 & 0 . \\ 1.1924 E-00 & 0 . \\ 1.3031 E 00 & 0 .\end{array}$

$\begin{array}{llll}\text { SGSO }(N, N) & S G S 1(N, N) & S G S O(N, N+1) & S G S 1(N, N+1) \\ 2.9362 E-01 & 4.5037 E-03 & 8.9885 E-03 & -2.9959 E-03 \\ 3.3229 E-01 & 5.0250 E-03 & 9.9796 E-03 & -3.3511 E-03 \\ 3.6901 E-01 & 5.3656 E-03 & 1.0726 E-02 & -3.5671 E-03 \\ 3.8232 E-01 & 5.5708 E-03 & 1.0970 E-02 & -3.7317 E-03 \\ 3.8585 E-01 & 5.6938 E-03 & 1.1216 E-02 & -3.8131 E-03 \\ 4.0268 E-01 & 6.0254 E-03 & 1.1938 E-02 & -4.0234 E-03 \\ 4.3779 E-01 & 6.7285 E-03 & 1.3383 E-02 & -4.4834 E-03 \\ 4.7816 E-01 & 7.5848 E-03 & 1.4970 E-02 & -5.0732 E-03 \\ 5.8649 E-01 & 4.8060 E-03 & 4.7761 E-03-1.5989 E-03 \\ 8.0497 E-01 & 6.9332 E-03 & 6.9147 E-03-2.2880 E-03 \\ 9.8790 E-01 & 8.3942 E-03 & 8.3479 E-03 & -2.7871 E-03 \\ 1.1285 E-00 & 9.1267 E-03 & 9.0652 E-03 & -3.0401 E-03 \\ 8.8428 E-01 & 7.7594 E-03 & 7.7308 E-03 & -2.5653 E-03 \\ 5.3328 E-01 & 6.4491 E-03 & 7.7269 E-03 & -2.5501 E-03 \\ 5.0098 E-01 & 7.4409 E-03 & 1.1176 E-02-3.6810 E-03 \\ 4.7855 E-01 & 7.2537 E-03 & 1.0857 E-02 & -3.6006 E-03 \\ 4.6516 E-01 & 6.9393 E-03 & 1.0386 E-02 & -3.4452 E-03 \\ 4.5312 E-01 & 6.4754 E-03 & 9.4278 E-03 & -3.1293 E-03 \\ 4.0542 E-01 & 3.1647 E-03 & \end{array}$


*INCONEL X

\begin{tabular}{|c|c|c|c|c|}
\hline FORMULA & MASS FRACTION & ATOM & FORMULA & MASS FRACTION \\
\hline $4.1000000 E+00$ & $7.0000000 \mathrm{E}-\mathrm{OI}$ & CR & $4.2000000 E+00$ & I. $5500000 \mathrm{E}-01$ \\
\hline $4.0000000 E+00$ & $7.4000000 \mathrm{E}-02$ & TI & $4.3000000 \mathrm{E}+00$ & $2.5000000 \mathrm{E}-\mathrm{C}$ \\
\hline & $1.0000000 \mathrm{E}-02$ & $\mathrm{MN}$ & $4.4000000 E+00$ & $1.0000000 \mathrm{E}-\mathrm{C}$ \\
\hline $5.3600000 \mathrm{E}+\infty 0$ & $9.0000000 \mathrm{E}-03$ & AL & 5. $1.000000 \mathrm{E}+00$ & $7.0000000 \mathrm{E}-\mathrm{C}$ \\
\hline $8.3600000 E+00$ & $5.0000000 E-03$ & SI & $5.5000000 E+00$ & $5.0000000 \mathrm{E}-\mathrm{C}$ \\
\hline $\begin{array}{c}\text { FORMULA } \\
F\end{array}$ & $\begin{array}{c}\text { DENSITY } \\
\text { RHO }(G / C C)\end{array}$ & $\begin{array}{l}\text { FUEL } \\
\text { RHOF }\end{array}$ & $\begin{array}{l}\text { DENSITY } \\
(G / C C)\end{array}$ & $\begin{array}{l}\text { TEMPERATURE } \\
T(D E G \quad K)\end{array}$ \\
\hline & $8.3099978 \mathrm{E} 00$ & 0 & & $2.9316000 E \quad 02 \quad 10$ \\
\hline
\end{tabular}

PROBABILITY/PATH (/CM)

\begin{tabular}{|c|c|c|c|c|c|}
\hline $\begin{array}{l}\text { COLLIDE } \\
\text { SG(N) }\end{array}$ & $\begin{array}{l}\text { MULTIPLY } \\
\text { NUSGF }(N)\end{array}$ & $S G S O(N, N)$ & $\operatorname{SGSI}(N, N)$ & $\begin{array}{l}\text { TER } \\
\text { SGSO }(N, N+I)\end{array}$ & $\operatorname{SGS1}(N, N+1)$ \\
\hline $\begin{array}{l}.3405 E-01 \\
.5158 E-01 \\
.6213 E-01 \\
.5788 E-01 \\
.6431 E-01\end{array}$ & $\begin{array}{l}0 . \\
0 . \\
0 . \\
0 . \\
0 .\end{array}$ & $\begin{array}{l}2.1585 E-01 \\
2.3184 E-01 \\
2.4094 E-01 \\
2.3704 E-01 \\
2.4336 E-01\end{array}$ & $\begin{array}{l}8.6331 E-03 \\
9.2660 E-03 \\
9.7549 E-03 \\
9.6127 E-03 \\
9.8863 E-03\end{array}$ & $\begin{array}{l}1.7378 E-02 \\
1.8585 E-02 \\
1.9457 E-02 \\
1.9194 E-02 \\
1.9914 E-02\end{array}$ & $\begin{array}{l}-4.9520 E-03 \\
-5.1805 E-03 \\
-5.1259 E-03 \\
-5.1013 E-03 \\
-5.5880 E-03\end{array}$ \\
\hline $\begin{array}{l}2.9224 E-01 \\
3.5578 E-01 \\
4.2816 E-01 \\
6.9166 E-01 \\
1.3113 E \quad 00\end{array}$ & $\begin{array}{l}0 . \\
0 . \\
0 . \\
0 .\end{array}$ & $\begin{array}{l}2.6777 E-01 \\
3.2753 E-01 \\
3.9763 E-01 \\
6.7910 E-01 \\
1.2874 E 00\end{array}$ & $\begin{array}{l}1.1100 E-02 \\
1.3127 E-02 \\
1.5005 E-02 \\
1.2243 E-02 \\
2.3050 E-02\end{array}$ & $\begin{array}{l}2.2104 E-02 \\
2.6289 E-02 \\
3.0370 E-02 \\
1.2243 E-02 \\
2.3049 E-02\end{array}$ & $\begin{array}{l}-5.4684 E-03 \\
-7.1020 E-03 \\
-9.8811 E-03 \\
-3.9821 E-03 \\
-7.5000 E-03\end{array}$ \\
\hline $\begin{array}{ll}1.5487 \mathrm{E} & 00 \\
1.3677 \mathrm{E} & 00 \\
1.3389 \mathrm{E} & 00 \\
1.3471 \mathrm{E} & 00 \\
1.3470 \mathrm{E} & 00\end{array}$ & $\begin{array}{l}0 . \\
0 . \\
0 . \\
0 .\end{array}$ & $\begin{array}{ll}1.5194 E & 00 \\
1.3384 E & 00 \\
1.3017 E & 00 \\
1.2870 E & 00 \\
1.2456 E & 00\end{array}$ & $\begin{array}{l}2.6996 E-02 \\
2.3555 E-02 \\
2.2865 E-02 \\
2.5215 E-02 \\
2.9533 E-02\end{array}$ & $\begin{array}{l}2.6995 E-02 \\
2.3547 E-02 \\
2.2856 E-02 \\
3.0310 E-02 \\
4.4542 E-02\end{array}$ & $\begin{array}{l}-8.7859 E-03 \\
-7.6724 E-03 \\
-7.4498 E-03 \\
-2.8823 E-03 \\
-1.4511 E-02\end{array}$ \\
\hline $\begin{array}{ll}1.3688 E & 00 \\
1.3530 E & 00 \\
1.3928 E & 00 \\
1.4986 E & 00\end{array}$ & $\begin{array}{l}0 \\
0 \\
0 \\
0 .\end{array}$ & $\begin{array}{ll}1.2311 E & 00 \\
1.1572 E & 00 \\
1.0972 E & 00 \\
1.1658 E & 00\end{array}$ & $\begin{array}{l}2.9214 E-02 \\
2.7476 E-02 \\
2.5356 E-02 \\
1.3602 E-02\end{array}$ & $\begin{array}{l}4.4035 E-02 \\
4.1429 E-02 \\
3.7201 E-02\end{array}$ & $\begin{array}{l}-1.4363 E-02 \\
-1.3505 E-02 \\
-1.2120 E-02\end{array}$ \\
\hline
\end{tabular}

RELIABILITY LIMITATION: COBALT CONTENT MOCKED UP WITH MANGANESE, BY ATOMS. FIRSTSIGNIFICANT-FIGURE UNCERTAINTY IN HIGH ENERGY SCATTER CROSS SECTIONS OF ALUMINUM CONIENT. 


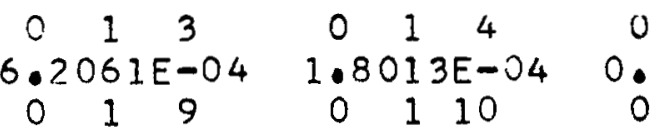
0

0.115

0

0.5

$3.7144 E-04$ O 211

0.

$\begin{array}{lll}0 & 2 & 17\end{array}$

0

038

$1.5868 E-04$

O 314

0.46

$7.1607 E-04$

0412

0

0

0

0 .

$0 \quad 517$

0.611

0.617

0.712

0.718

0.814

0.911

$\begin{array}{rrr}0 . & & \\ 0 & 9 & 17\end{array}$

0.1015

0.1114

0.1214

0.1315

$0.14 \quad 17$

ก. 1618
026

$1.3872 E-04$

0212

0.218

0.

039

$1.2993 E-04$

0315

0.
0

$4.3432 E-C 4$

0413

- 419

$\begin{array}{ccc}0 . & & \\ 0 & 512\end{array}$

0.518

0.
0

$\begin{array}{rrr}0 . & & \\ 0 & 6 & 18\end{array}$

$\begin{array}{rrr}0 & 6 & 18 \\ 0 & 7 & 13\end{array}$

0 .

0719

0.

$0 \quad 815$

0.

0912

0.918

0.
0 1016

0.1115

0.1316

$\begin{array}{rrr}0 . & & \\ 0 & 1418\end{array}$

0.
0
0619
16

0.1115
0.1115

$$
0
$$

0.

027

1. $3085 E-07$

0

0219

0 .

0310

0.

0316

0.48

$2.6343 E-04$

0414

0.

D 57

8. 3829E-04

0513

0.

0519

0.613

0.619

0.714

0 .

o 810

0 .

0816

0.913

0.919

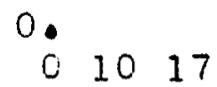

0.1116

0.11
01216

0.1317

0.

$0 \quad 1419$

0.1719
0
0.
0
0
0
0
0
0

0 .

0

214

035

$7 \cdot 1116 E-04$

0311

0 .

0317

0.

049

$1.6777 \mathrm{E}-04$

0415

0 .

$\begin{array}{lll}0 & 5\end{array}$

1. $2008 E-04$

0514

0 .

068

$1.1903 E-03$

C 614

0 . 7

0
$1.8410 E-03$

0715

0.811

$\begin{array}{rll}0 & 8 & 11 \\ 0 . & & \\ 0 & 8 & 17\end{array}$

0.914

0.1012

o. 1018

0.1117

0.1217

0 .

01318

0.
0.1517

0 $\begin{array}{llllll}0 & 1 & 7 & 0 & 1\end{array}$

$\begin{array}{rrrr}0.0 & 113 & 0 & 1 \\ 0 & 14\end{array}$

$\begin{array}{rrrrr}0.0 & 1 & 0 & & \\ 0 & 19 & 0 & 24\end{array}$

$0 . \quad 6.1240 E-04$

0290210

0.00

0216

0.0.

$\begin{array}{llllll}0 & 3 & 0 & 3\end{array}$

4. $3134 E-04$

0312

0.

0

0.

0

318

$2.6162 E-04$

0313

0.

0319

0 .

0416

0.

0

410

0.

0411

0

417

0.

0510

0

515

0.

059

$1.0860 E-03$

0615

0.710

0

710

0

$0 \quad 812$

0.

0516

0.

0010

0616

0.

0711

0.717

0.

0813

0.518

0.

0819

0.915

0.

0916

0.1013

0.

01014

0.

01019

0.

01113

0 .

01118

01119

0.
0 1218

0 .

01219

0 .

01319

0 .

01416

0.

01518

0 .

01519 
*INCONEL X

SCATTER

L. N1 N2

*INCONEL X

$S G S L(N 1, N 2)$

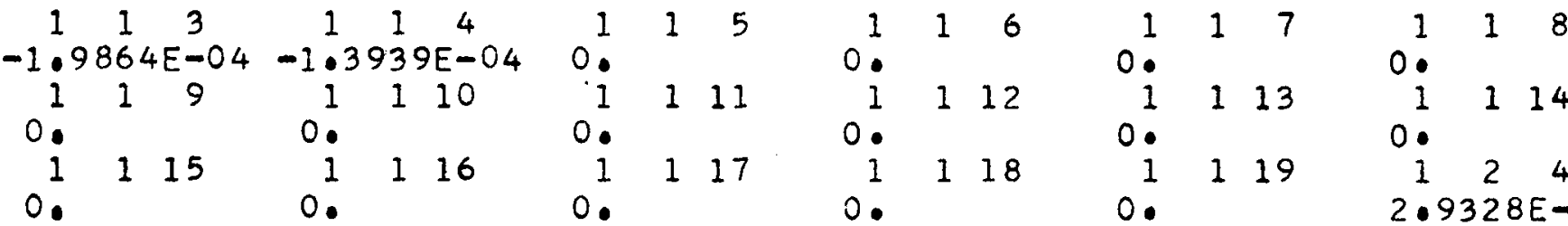
$\begin{array}{llllllllllllllllll}1 & 2 & 5 & 1 & 2 & 6 & 1 & 2 & 7 & 1 & 2 & 8 & 1 & 2 & 9 & 1 & 2 & 10\end{array}$

$-1.6102 E-04-1.1147 E-04-1.3032 E-07$ $\begin{array}{lllllllll}1 & 2 & 11 & 1 & 2 & 12 & 1 & 2 & 13 \\ 0 . & & 0 . & & 0 . & & \end{array}$

0.0

$\begin{array}{llllll}1 & 2 & 15 & 1 & 2 & 16\end{array}$

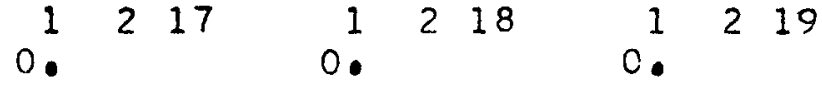

$\begin{array}{lllllllll}1 & 3 & 5 & 1 & 3 & 6 & 1 & 3 & 7\end{array}$

$\begin{array}{lllllll}1 & 3 & 8 & 1 & 3 & 9 & 1\end{array}$

$-5.8404 E-05-9.2352 E-050$.

$\begin{array}{lllllll}1 & 3 & 14 & 1 & 3 & 15 & 0\end{array}$

$2.9269 E-04 \quad 6.3726 E-05 \quad-2.7942 E-05$

$\begin{array}{llllllllll}1 & 3 & 11 & 1 & 3 & 12 & 1 & 3 & 13\end{array}$

0 . 0 .

$\begin{array}{lllllllll}1 & 3 & 17 & 1 & 3 & 18 & 1 & 3 & 19\end{array}$

0 - 0 0.

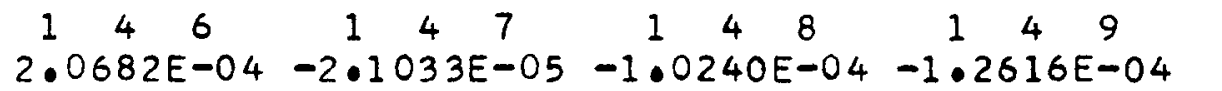

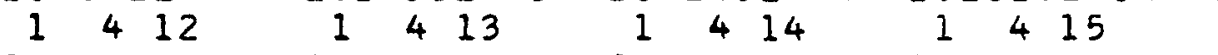

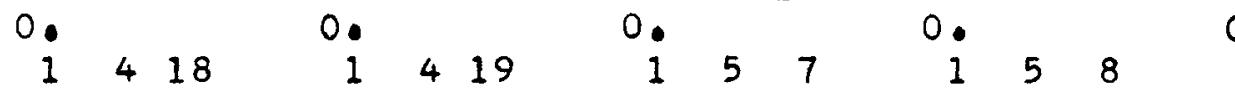

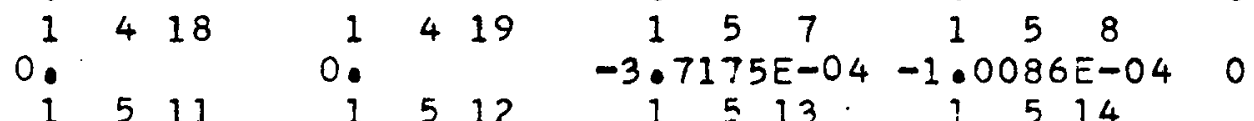

$1410 \quad 1411$

0.0

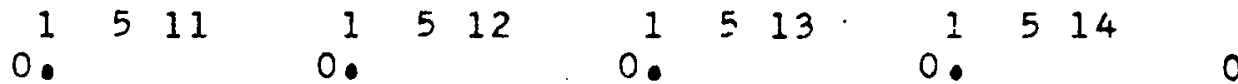

$\begin{array}{lllll}4 & 16 & 1 & 4\end{array}$

1517

1518

1519

1

1

5

1

0.611

c.

0. 617

1612

0.613

1.16898

1

51

$1 \cdot 1689 E-04-5.3171 E-04$

1510

0.712

1618

0. 619

1614

1615

0.

1 79

0.

$-4.9365 E-040$.

i 714

1715

1

710

1516

$\begin{array}{lll}1 & 712 \\ 0 . & & \end{array}$

1713

0.1810

0 .

1

716

1610

1718

1719

$\begin{array}{lll}1 & 8 & 10\end{array}$

1811

0.

1816

1817

1

716

1814

1815

0.

0.

19914

0.

$8 \quad 12$

1

818

0.

1915

$110 \quad 12$

0.

11013

0.

0.

11018

11019

1616

1
0.

0.11016

0.

$\begin{array}{lll}1 & 10 & 17\end{array}$

0.

0. 1114

11115

11116

$\begin{array}{lll}1 & 11 & 17\end{array}$

0. 1214

0.1215

0.1216

0.

11217

0. 1317

0.

0.1315

0.

0

11316

11318

0.

11118

0.

11218

0 .

11319

0.

$\begin{array}{llllll}1 & 14 & 17 & 1 & 14 & 18\end{array}$

) 16.18

0.

0.

$\begin{array}{lll}1 & 15 & 17\end{array}$

11419

0.

$\begin{array}{lll}1 & 15 & 18\end{array}$

0.

0 .

0 .

1711

0.717

0.813

0.

1819

0. 916

0.

$\begin{array}{lll}1 & 10 & 14\end{array}$

0 .

11113

0.

11619

11719

0.

11119

11219

0.

$114 \quad 16$

0.

11519

0. 


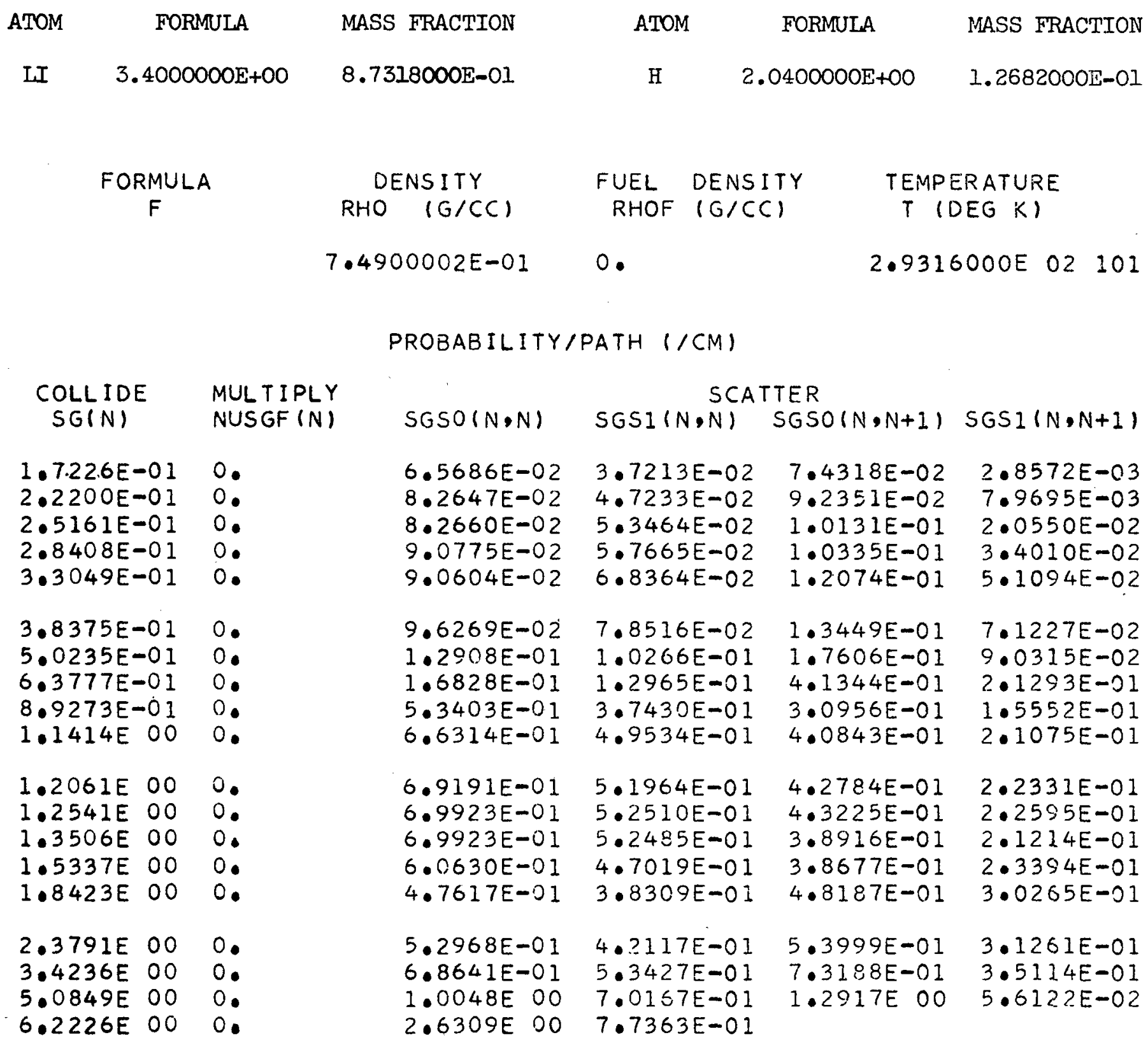


$01 \frac{3}{1}$

0 . $9123 E-0$

$1.3415 E-03$

0115

$-6.2265 E-08$

025

$1.0994 E-02$

0211

$5.9852 E-05$

0.217

0 .

038

$5.7693 E-03$

0314

$3.6107 E-07$

046

$3.5223 E-02$

0412

$4.2792 E-05$

0418

0.
0

$6.7884 E-04$

C 517

2.9102E-07

0611

$1.4316 E-03$

0617

$7.4447 E-08$

0712

$4.1529 E-04$

0718

$9.6781 E-08$

0814

$1.4332 E-05$

0911

4.0068E-02

O 917

2.2187E-06

01015

$1.6072 E-04$

$0 \quad 1114$

$6.9064 E-03$

O 1214

$5.1612 E-02$

01315

$6.9239 E-02$

01417

$5.0938 E-02$

O 1618

$1.9893 E-01$
014

$7.4371 E-03$

0110

$1.8156 E-04$

0116

026

$6.6682 E-03$

0212

$8.1229 E-06$

0218

0.

039

$7.6897 E-03$

0315

$6.5649 E-08$

047

$2.1341 E-02$

0413

$5.8516 E-06$

0419

0.512

$9.1934 E-C 5$

0518

$-2.3282 E-C 7$

$0 \quad 6 \quad 12$

$1.9371 E-04$

0618

0.

0713

$5.6327 E-0.5$

0719

0.815

$2.4500 E-16$

0912

$5.4227 E-C 3$

0918

$8.0251 E-07$

01016

$5.9214 E-05$

01115

$1.2539 E-03$

01215

$9.3705 E-03$

01316

$2.5472 E-02$

C 1418

$1.9323 E-02$

01619

$9.9641 E-02$
015

4.5108E-03

0111

$2.4587 E-05$

० 117

0.

$\begin{array}{ll}0 & 2\end{array}$

4.0444E-03

O 213

$1.1275 E-06$

0219

0.

0310

$1.0407 E-03$

0316

0.

048

$1.2944 E-02$

0414

$6.2536 E-07$

057

4.7842E-02

O 513

$1.2397 E-05$

$0 \quad 519$

0.

0613

$2.6057 E-05$

0619

0 .

0714

$6.6779 E-06$

$081 \mathrm{C}$

4.7315E-02

O 816

$8.5750 E-07$

0913

$7.3378 E-04$

0919

$3.7765 E-17$

$\begin{array}{lll}0 & 10 & 17\end{array}$

$2.1797 E-05$

01116

4.6113E-04

01216

$3.4473 E-03$

$\begin{array}{lll}0 & 1317\end{array}$

$9.3705 E-03$

01419

$1.0321 E-02$

C 1719

$2.3885 E-01$
016

$2.7359 E-0$

$3.3156 E-06$

0118

0.128

$2.4531 E-03$

0214

$9 \cdot 2044 E-08$

035

2.7405E-02

0311

1.4083E-04

0317

0 .

049

I. $7 \angle 53 E-02$

0415

$1.3401 E-07$

058

$2.7808 E-02$

O 514

$1.5133 E-06$

068

$6.1779 E-02$

0614

$3.3501 E-06$

079

$1.6995 E-01$

0715

$1.3549 E-06$

0811

$6.4033 E-03$

0817

$3.6750 E-07$

0914

$8.9267 \varepsilon-05$

01012

$5.3783 E-02$

01018

$8.174 I E-06$

01117

1.6970E-04

$0 \quad 1217$

1.2682E-03

01318

$3.5547 \mathrm{E}-03$

C 1517

1. $7154 E-01$ $\begin{array}{lll}0 & 1\end{array}$

$1.6594 E-03$

0113

4.5142E-07

0119

0.29

$3.2696 E-03$

0215

4.6022E-08

036

1. 56825-02

0312

$1.9104 \mathrm{E}-05$

0318

0 .

0410

$2.3349 E-03$

0416

$2.2334 E-07-1.3401 E-07$

$\begin{array}{llll}0 & 5 & 0 & 510\end{array}$

$3.7065 E-02 \quad 5.0162 E-03$

$0 \quad 515$

$2.9102 E-07$

069

$7.8163 E-02$

0615

5.9558E-07

0710

$2.2673 E-02$

0716

4.8391E-07

0812

$8.6650 E-04$

0818

6.1250E-07 -

0915

$1.6239 E-05$

01013

$7.2787 E-03$

01019

4. $3088 \mathrm{E}-06$

01118

$6.4442 E-05$

01218

4.8093E-04

01319

1.8985E-03

01518

$6.5072 E-02$

O 516

$5.8204 \equiv-08$

0610

$1.0578 E-02$

0616

$2.2334 E-07$

0711

$3.0685 E-03$

0717

0.

0813

$1.1742 E-04$

0819

$-3.67505-07$

0916

5.9480E-06

01014

$3.8492 E-04$

01113

$5.6799 E-02$

01119

$3.4329 E-05$

01219

$2.5698 E-04$

01416

$1.3846 E-01$

01519

$3.4502 E-0 ?$ 
131

113 $6.8565 E-03$ 119

$1.5949 E-04$ 1115

$2.7465 E-07$ 125

$5.3383 E-03$

1211

$2.8563 E-06$

$\begin{array}{lll}1 & 2 & 17\end{array}$

0.38

$1.7037 E-03$

1314

$1.0951 E-07$

146

$2.1876 E-02$

1412

$1.8178 E-06$

1418

1 511

$3.3994 E-05$

1517

$-1.5774 \mathrm{E}-07$

1611

8.6091E-05

1617

$4.9663 E-08$

$\begin{array}{lll}1712 & 712\end{array}$

$1.6658 E-05$

1718

$2.5826 E-07$

1814

$1.2258 E-06$

1911

$7.9842 E-03$

1917

4.0939E-07

11015

$6.3406 E-06$

11114

5.5885E-04

11214

1.0766 E-02

11315

$1.9518 \mathrm{E}-02$

11417

$1.1787 \mathrm{E}-02$

11618

4. $7220 E-02$ $\begin{array}{lcc}1 & 1 & 4 \\ 3.611 & 15 E-C 3\end{array}$

1110

$1.0129 E-05$

1116

0.

126

$2.5252 E-03$

1212

$7.0640 E-07$

1218

0

139

$1.4606 \mathrm{E}-133$

1315

$4.3802 E-08$

147

$1.0357 E-02$

1413

$4.4699 E-07$

1419

0 .

1512

$3.5139 E-06$

1518

$2.7422 E-07$

1612

$7.1515 \mathrm{E}-06$

1618

$1.4899 \mathrm{E}-\mathrm{C} 7$

1713

$2.8086 E-06$

1719

1815

$3.2689 E-07$

1912

$4.2137 E-04$

1918

$2.8342 E-07$

11016

$3.0858 E-06$

I 1115

$5.9797 E-05$

11215

$1.0090 E-C_{3}$

11316

4.3077E-03

11418

2. $6898 E-03$

11619

$-2.0238 E-02$
$1 \stackrel{1}{5}$

1111

$1.3142 E-06$

$\begin{array}{lll}1 & 117\end{array}$

0.

127

$1.1957 E-03$

1213

$2.1499 E-07$

1219

0.

1310

$7.8493 E-05$

1316

$1.0951 E-07$

148

4.8968E-03

1414

$2.0859 E-07$

157

$2.6859 E-02$

1513

$7.7655 E-07$

I 519

0.

1613

$1.4899 E-06$

1619

i 714

$6.4566 E-07$

1810

$1.1469 E-02$

1816

$1.6345 E-07$

1913

$2.9759 E-05$

1919

$5.3535 E-07$

11017

$1.4795 E-06$

11116

$1.7812 E-05$

I 1216

$2.3737 E-04$

11317

$9.3246 E-04$

I 1419

$6.9541 E-04$

11719

$-5.9359 E-02$
116

8.0907E-04

1112

$3.7400 E-07$

$\begin{array}{lll}1 & 1 & 18\end{array}$

0.

128

$5.6698 E-04$

1214

$6.1426 E-08$

135

$1.4738 E-02$

1311

6.0447E-06

1317

0 .

149

4.1818E-03

1415

$2.9799 E-08$

158

$1.3494 E-02$

1514

$2.3297 E-07$

168

$3.4018 E-02$

1614

2.9798E-07

179

$8.3506 E-02$

1715

4.5196E-07

1811

$5.9425 E-04$

1817

2.4517 E-07

1914

4.0466E-06

$110 \quad 12$

$1.0718 E-02$

11018

$9.7223 E-07$

11117

6.7408E-06

$\begin{array}{lll}1 & 1217\end{array}$

6.0387E-05

11318

$1.9448 E-04$

$115 \quad 17$

$6.4302 E-02$

$\begin{array}{llllll}1 & 1 & 7 & 1 & 1 & 8\end{array}$

$3.8374 E-04 \quad 1.8248 E-04$

$\begin{array}{llllll}1 & 1 & 13 & 1 & 1 & 14\end{array}$

$1.2467 E-07-1.7077 E-07$

1119124

$0.1 .0697 E-02$

1291210

4.8997E-04 2.8333E-05

$\begin{array}{llllll}1 & 2 & 15 & 1 & 2 & 16\end{array}$

$1.0750 E-07 \quad 0$.

$\begin{array}{llllll}1 & 3 & 6 & 1 & 3 & 7\end{array}$

$7.6123 E-03 \quad 3.5999 E-03$

1312

$1.0732 E-06$

1318

0.

1410

$2.1654 E-04$

1415

$-1.9742 E-07$

159

$1.1505 E-02$

1515

$7.7655 E-08$

169

$3.1108 E-02$

1615

$1.9865 E-07$

1710

4.2985E-03

1716

$6.4566 E-08$

1812

$3.8165 E-05$

1818

$-5.5674 E-07$

1915

$1.1652 E-06$

$\begin{array}{lll}1 & 10 & 13\end{array}$

5.6615E-04

11019

$1.6486 E-06$

11118

$3.3035 E-06$

11218

$1.8443 E-05$

11319

$1.6837 E-05$

11518

l. $2014 E-02$

1313

$3.2852 E-07$

1319

0 .

1411

$1.3886 E-05$

1417

$3.4642 E-07$

1510

$5.8590 E-04$

1516

$7.7655 E-08$

1610

$1.5702 E-03$

1616

4. $9663 E-08$

1711

$2.2845 E-04$

1717

$6.4566 E-08$

1813

5.0260E-06

1819

$8.8363 E-07$

1916

$6.9281 E-07$

11014

$3.6268 E-05$

11113

$1.1320 E-02$

11119

4.2856E-06

I 1219 
*L I THIUM OXIDE

*L120

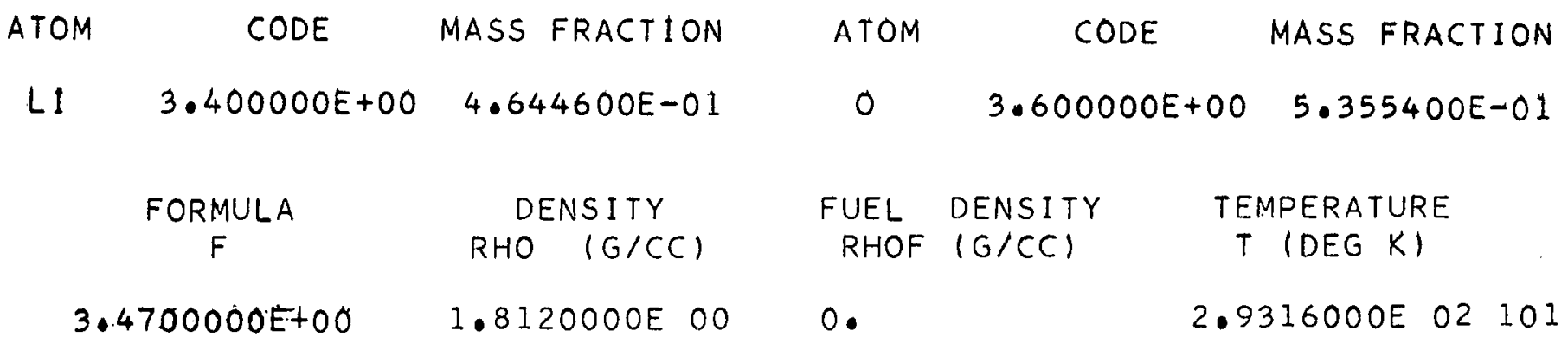

PROBABILITY/PATH (/CM).

\section{COLLIDE MULTIPLY $S G(N)$

\begin{abstract}
NUSGF $(N)$
\end{abstract}
$1.6249 E-01$

$2.2176 E-01$

$2.0762 E-01$

$1.9315 E-01$

$2.5780 E-01$

$2.6314 E-01$

$2.6563 E-01$

$2.9651 E-01$

$2.6660 E-01$

$2.3768 \mathrm{E}-01$

$2.4418 E-01$

$2.8936 E-01$

4. $1292 E-01$

$6.2923 E-01$

$1.0027 E 00$

$1.5121 E 00$

$2.3529 E \quad 00$

$3.7967 E \quad 00$

$4.8329 E \quad 00$
0.

0.

0.

0.

0.

0.

0.

0.

0.

0.

0.

0.

0.

0 .

0.

0.

0.

0.

0 .
SCATTER

SGSO $(N, N) \quad$ SGSI(N,N) SGSO $(N, N+1) \quad S G S I(N, N+1)$

$8.5462 E-02$

1. 2560E-01

1.1488E-01

I. $1752 \mathrm{E}-01$

$1.6087 E-01$

$3.4152 E-02$

4. $3202 E-02$

4.0835E-02

$3.4493 E-02$

4.284OE-02

$1.6804 E-01$

$1.6214 E-01$

4. 1407E-02

4. $5224 E-02$

$1.7955 E-01$

$2.3738 E-01$

$2.0549 E-01$

5. $1989 E-02$

$2.5614 E-02$

$2.2429 E-02$

1. $9896 E-01$

1. $9905 E-01$

1. $9905 E-01$

$1.9295 E-01$

$1.8746 E-02$

$1.8288 E-02$

$1.8288 \mathrm{E}-02$

2.0031E-02

$1.8075 E-01$

$2.3517 E-02$

$1.8075 E-01$

$2.3517 E-02$

$1.8155 E-01$

$1.8908 E-01$

$2.3589 E-02$

$2.3856 E-02$

$2.3242 E-01$
$7.5625 E-02-1.8951 E-02$

$9.4954 E-02-2.4929 E-02$

$9.0254 E-02-2.1903 E-02$

$7.4947 E-02-2.0968 E-02$

$9.3552 E-02-2.2812 E-02$

$9.0026 E-02-2.0407 E-02$

$9.9037 E-02-2.3501 E-02$

$1.1525 E-01-3.0893 E-02$

$2.5643 E-02-7.0751 E-03$

$2.2460 E-02-6.0817 E-03$

$1.8762 E-02-5.3209 E-03$

$1.8303 E-02-5.2285 E-03$

$1.8303 E-02-5.2285 E-03$

$2.4404 E-02-6.9714 E-03$

$3.6606 E-02-1.0457 E-02$

$3.6606 E-02-1.0457 E-02$

$3.6716 E-02-1.0491 E-02$

$3.6051 E-02-1.0273 E-02$ 
SGSL (NI,N2)

$\begin{array}{llll}0 & 1 & 3 & 0\end{array}$

$1.1085 E-03 \quad 0$.

01

0.1150

$0 . \quad 0$.

02520

0.211

0

0.

0

0

0

0

046

4.7907E-05

0412

0.

0

418

0

0 .

0

0.

0

0

0.

0

0 .

0

0814

$\begin{array}{rrr}0 . & & \\ 0 & 9 & 11\end{array}$

$\begin{array}{rrr}0 . & & \\ 0 & 9 & 17\end{array}$

0.
0 1015

0 .

01114

0.1214

0.1315

0 .

01417

0.1618

0 . $\begin{array}{rrr}0 . & 6 \\ 0 & 2 & 12\end{array}$

0.

0218

0.

039

0.315

0.

047

0.

0413

0 .

0419

0.512

0.

0518

0

0612

0

0618

0. 713

0.719
07

0

$0 \quad 8 \quad 15$

0 .

0912

0.

o 918

$\begin{array}{rrr}0 . & & \\ 0 & 1016\end{array}$

0
0 1115

$\begin{array}{rrr}0 . & & \\ 0 & 12 & 15\end{array}$

0.
0 1316

0 .

0 1418

0.1619

0 .
015

0.

0111

0.117

0.

027

0.

0213

0.

0219

0.

0310

0 .

0316

0.

$\begin{array}{lll}0 & 4 & 8\end{array}$

0.

0414

0.57

2. $5666 \mathrm{E}-03$

0513

0.519

0.013

0.619

$\begin{array}{rrr}0 . & & \\ 0 & 7 & 14\end{array}$

$\begin{array}{rrr}0 . & & \\ 0 & 8 & 10\end{array}$

$\begin{array}{rrr}0 . & & \\ 0 & 8 & 16\end{array}$

0.

0913

0.919

0.

$\begin{array}{lll}0 & 10 & 17\end{array}$

0.

01116

$\begin{array}{rll}0 . & & \\ 0 & 1216\end{array}$

0.1317

0

014,19

0.

01719

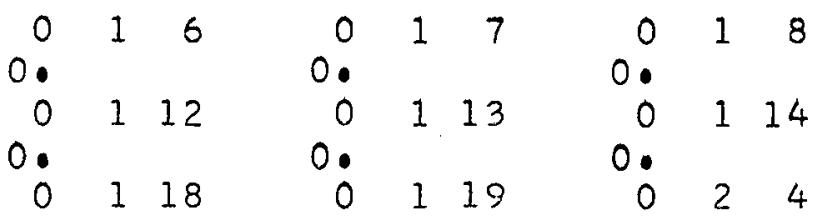

0.18

$\begin{array}{llllll}0 & 2 & 8 & 0 & 2\end{array}$

0.214

0.35

$1.9926 E-03$

0311

$\begin{array}{rrr}0 . & & 11 \\ 0 & 3 & 17\end{array}$

0.49

$\begin{array}{rrr}0 & 4 & 9 \\ 0 . & & \end{array}$

0.415

058

0.514

0 .

068

4.0361E-03

0614

0.

079

3. $1174 E-03$

0715

0.811

0.

0817

0.

0914

0.1012

0.1018

$\begin{array}{rrr}0 . & & \\ 0 & 11 & 17\end{array}$

0.1217

01318

$\begin{array}{rrr}0 . & & \\ 0 & 15 & 17\end{array}$

0 . $\begin{array}{rrr}0 . & & \\ 0 & 2 & 15\end{array}$

0.

036

0312

0.318

0.

0410

0 .

0416

0.59

0.515

0.

069

0.615

0.
0

0.

0716

0.812

0.18

0

0.

0

01013

0.

$0 \quad 1019$

0 .

01118

0 .

0 12. 18

0.

01319

0 .

01518

0.
$8.2527 E-04$

0210

0.16

$\begin{array}{lll}0 . & & \\ 0 & & \end{array}$

0.313

0.

0319

0 .

0411

0

0.510

0.516

0.610

0616

0.711

$\begin{array}{rrr}0 . & & \\ 0 & 717\end{array}$

0813

0.819

0 .

0916

0.

$0 \quad 1014$

0 .

01113

0 .

01119

0.1219

0.1416

0 .

01519
0417

0. 
$\begin{array}{ccc}1 & 1 & 3 \\ -1.0065 E-03 & 0 \\ 0 & 1 & 9\end{array}$

$\begin{array}{llll}0 & 1 & 0 & 0\end{array}$

$0.15 \quad 1$

$\begin{array}{llll}1 & 2 & 5 & 1\end{array}$

1211

0

$\begin{array}{lll}1 & 2 & 17\end{array}$

0

0

1314

0 .

146

$-4.7011 E-05$

1412

$\because 418$

0.111

$0: 517$

0

16611

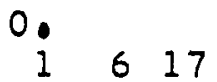

0.

1. 712

$\begin{array}{lll}0 & 7 & 12 \\ 0 & 7 & 18\end{array}$

$\begin{array}{lll}0 & 8 & 14\end{array}$

0019

0.

0.

$\begin{array}{lll}0 & 10 & 15\end{array}$

0.

11114

0.

11214

0.

11315

0.

$\begin{array}{lll}1 & 14 & 17\end{array}$

0.

11618
126

1212

$\begin{array}{lll}0 & 1 & 18\end{array}$

$\begin{array}{lll}0 & 1 & 3\end{array}$

$\begin{array}{lll}0 & 1 & \\ 0 & 3 & 15\end{array}$

$\begin{array}{lll}0 & & \\ 1 & 4\end{array}$

0.

1413

0.

1419

$\begin{array}{lll}0 & 1 & \\ 0 & 12\end{array}$

0.

1

1612

0.

$\begin{array}{lll}1 & 6 & 18\end{array}$

0.

1713

$0: 719$

$\begin{array}{lll}0 & 1 & \end{array}$

0.

1912

0.

1918

0.

11016

0.

11115

0.

11215

0.

11316

0.

11418

0.

11010
115

0.

1111

$\begin{array}{lll}0 & 1 & 17\end{array}$

0.

12

0.

1213

0.

1219

0

1310

0.

1316

0.

1

1414

0.

157

$-2.1786 E-03$

1513

0.

1519

0.

1613

0.

1

0.

1714

0.

1

0.

1

0.

1

0.

1

$\begin{array}{lll}1 & 10 & 17\end{array}$

0.

11116

0.

11216

0.

$\begin{array}{lll}1 & 13 & 17\end{array}$

0.

$\begin{array}{lll}1 & 14 & 19\end{array}$

0.

$1 \quad 17 \quad 19$
116

$\begin{array}{lll}0 & 1 & 12\end{array}$

$\begin{array}{lll}0 . & & 12 \\ 1 & 1 & 18\end{array}$

0.

128

0.

1214

0.

135

$-1.7575 E-03$

1311

$\begin{array}{lll}0 & & \\ 0 & 3 & 17\end{array}$

0

1

1415

0.

$\begin{array}{lll}1 & 5 & 8\end{array}$

1

514

0

168

1614

0.

179

$-2.6267 E-03$

1715

0.

1

0.

811

$\begin{array}{lll}1 & 8 & 17\end{array}$

0.

$\begin{array}{lll}1 & 9 & 14\end{array}$

0.

$\begin{array}{lll}1 & 10 & 12\end{array}$

0.

$\begin{array}{lll}1 & 10 & 18\end{array}$

0.

11117

0.

$\begin{array}{lll}1 & 12 & 17\end{array}$

0.

$\begin{array}{lll}1 & 13 & 18\end{array}$

$\begin{array}{lll}0 & 1517 \\ 0 & 15 & 17\end{array}$ $\begin{array}{llllll}1 & 1 & 7 & 1 & 1 & 8\end{array}$

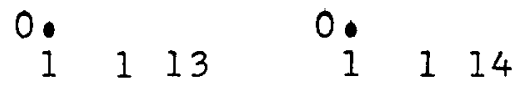

$\begin{array}{llllll}0 & 0.19 & & \\ 0 & 1 & 19 & \end{array}$

0.

129

0.

1215

0.

136

1

0.

1

312

318

1

410

0.

1416

0.

159

0.

1

0.

$\begin{array}{lll}1 & 6 & 9\end{array}$

$\begin{array}{lll}0 & 6 & 15\end{array}$

0.

1

1

0.

1

0

$\begin{array}{lll}1 & 8 & 18\end{array}$

0.

1915

0.

11013

0.

11019

0.

$\begin{array}{lll}1 & 11 & 18\end{array}$

0.

11218

0.

11319

$\begin{array}{lll}1 & 15 & 18\end{array}$

0
$-7.6416 E-04$

1210

0.

1216

0.37

0.13

0.319

0.411

0.

1417

$\begin{array}{rrr}0 & 1 & \\ 0 & 5 & 10\end{array}$

$\begin{array}{lll}0 & 1 & 16\end{array}$

0.

$16 \quad 10$

1616

0.

$\begin{array}{lll}1 & 7 & 11\end{array}$

$\begin{array}{lll}0.17 & 7 & 17\end{array}$

$\begin{array}{lll}0.1 & & 13\end{array}$

i $8 \quad 19$

0.916

0.

$\begin{array}{lll}1 & 10 & 14\end{array}$

0.

11113

$\begin{array}{lll}0 & 11 & 19\end{array}$

$\begin{array}{lll}0 & 12 & 19\end{array}$

$\begin{array}{lll}1 & 14 & 16\end{array}$

0.1519

0.

$$
0 \text {. }
$$

$$
0 \text {. }
$$$$
0 .
$$ 


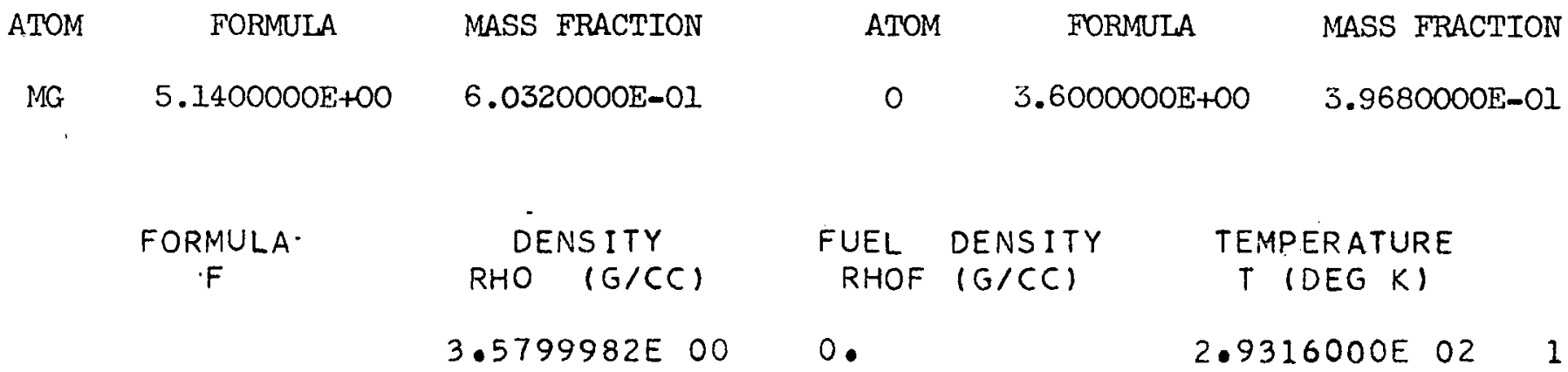

PROBABILITY/PATH (/CM)

\section{COLLIDE MULTIPLY $S G(N)$ \\ $\operatorname{NUSGF}(N)$}

$1.2329 E-01$

$1.8185 E-01$

$2.1101 E-01$

$2.4364 E-01$

$3.7386 E-01$

4.8057E-01

$5.7233 E-01$

$6.6729 E-0 I$

$5.3489 E-01$

$3.8243 E-0 I$

$3.8244 E-01$

$3.8247 E-01$

$3.8255 E-01$

$3.8269 E-01$

$3.8293 E-01$

$3.8327 E-01$

$3.8515 E-01$

3.8960E-01

$3.9352 E-01$
0

$\mathrm{C}$.

0

0 .

0.

0.

0 .

0.

0.

0.

0.

0 .

0.

0.

0.

0.

0.

0 .
SCATTER

SGSO (N,N) SGSI(N,N) SGSO(N,N+1) SGSI(N,N+I)

$1.0050 E-01 \quad 1.1014 E-02 \quad 2.2797 E-02-7.0870 E-03$

$1.4544 E-01 \quad 1.7537 E-02 \quad 3.6413 E-02-1.1248 E-02$

$1.6981 E-01 \quad 1.9860 E-02 \quad 4.1197 E-02-1.2750 E-02$

$1.9628 E-01 \quad 2.2835 E-02 \quad 4.7359 E-02-1.4663 E-02$

2.963IE-D 1 3.7298E-02 7.7544E-02 -2.3887E-02

$3.8294 E-01 \quad 4.6993 E-02 \quad 9.7626 E-02-3.0121 E-02$

4.6341E-01 5.2560E-02 1.0892E-01 -3.3780E-02

$5.4361 E-01 \quad 5.9747 E-02 \quad 1.2368 E-01-3.8442 E-02$

$5.0930 E-01 \quad 2.5582 E-02 \quad 2.5587 E-02-7.9316 E-02$

$3.6298 E-01 \quad 1.9442 E-02 \quad 1.9445 E-02$-5.9990E-03

$3.6298 E-01 \quad 1.9442 E-02 \quad 1.9445 E-02-5.9990 E-03$

$3.6298 E-01 \quad 1.9442 E-02 \quad 1.9445 E-02 \quad-5.9990 E-03$

$3.6298 E-01 \quad 1.9442 E-02 \quad 1.9445 E-02-5.9990 E-03$

$3.5649 E-01 \quad 2.1441 E-02 \quad 2.5927 E-02-7.9987 E-03$

$3.4353 E-01$ 2.5441E-02 3.8391E-02 -1.1998E-02

$3.4353 E-01 \quad 2.5441 E-02 \quad 3.8891 E-02-1.1998 E-02$

$3.4471 E-01 \quad 2.5546 E-02 \quad 3.9053 E-02-1.2047 E-02$

$3.4960 E-01 \quad 2.5353 E-02 \quad 3.7667 E-02-1.1612 E-02$

$3.9052 E-01 \quad 1.3984 E-02$ 
*NICKEL-CHROMIUM ALLOY

*NICKEL-CHROMIUM ALLOY

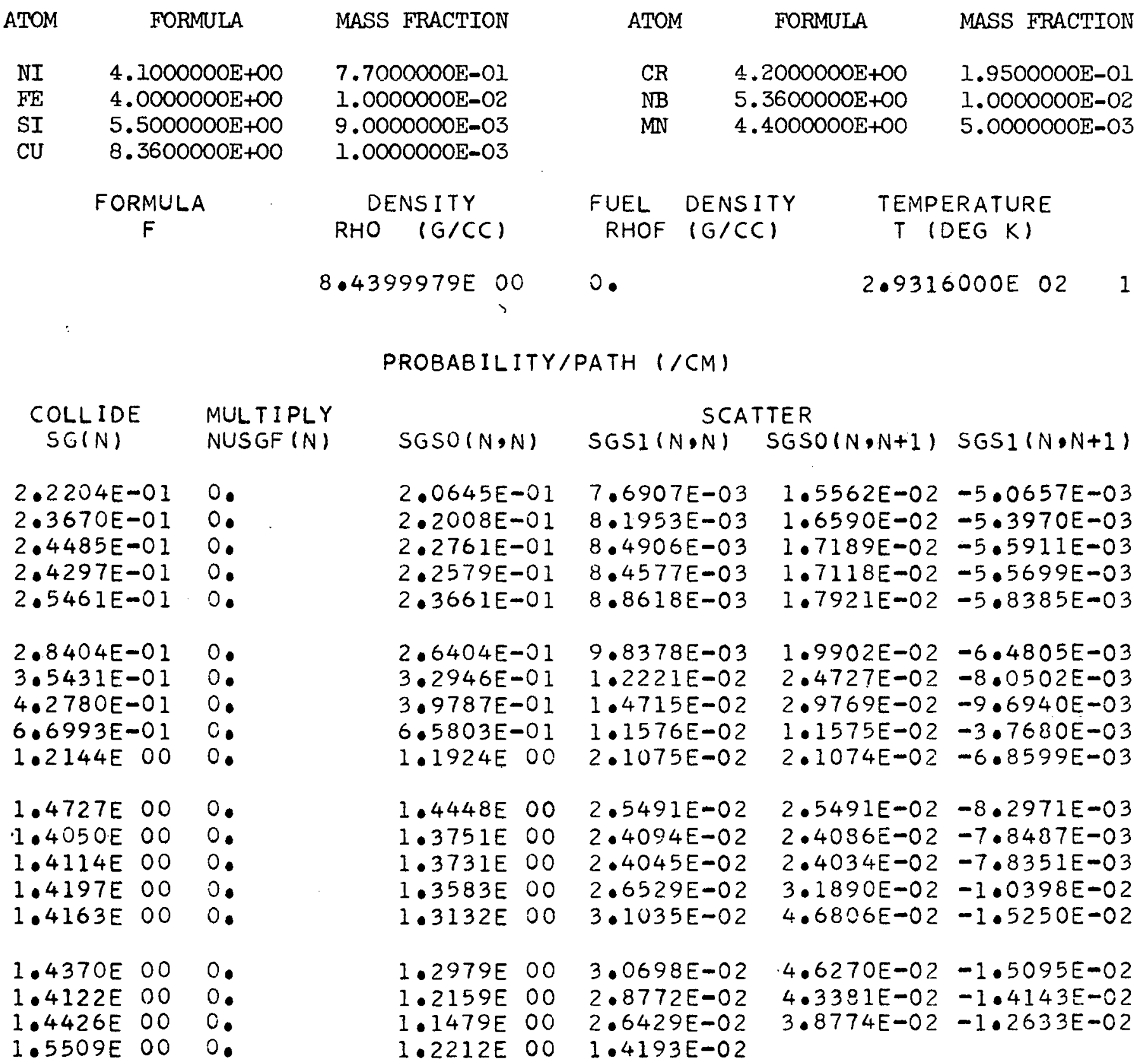




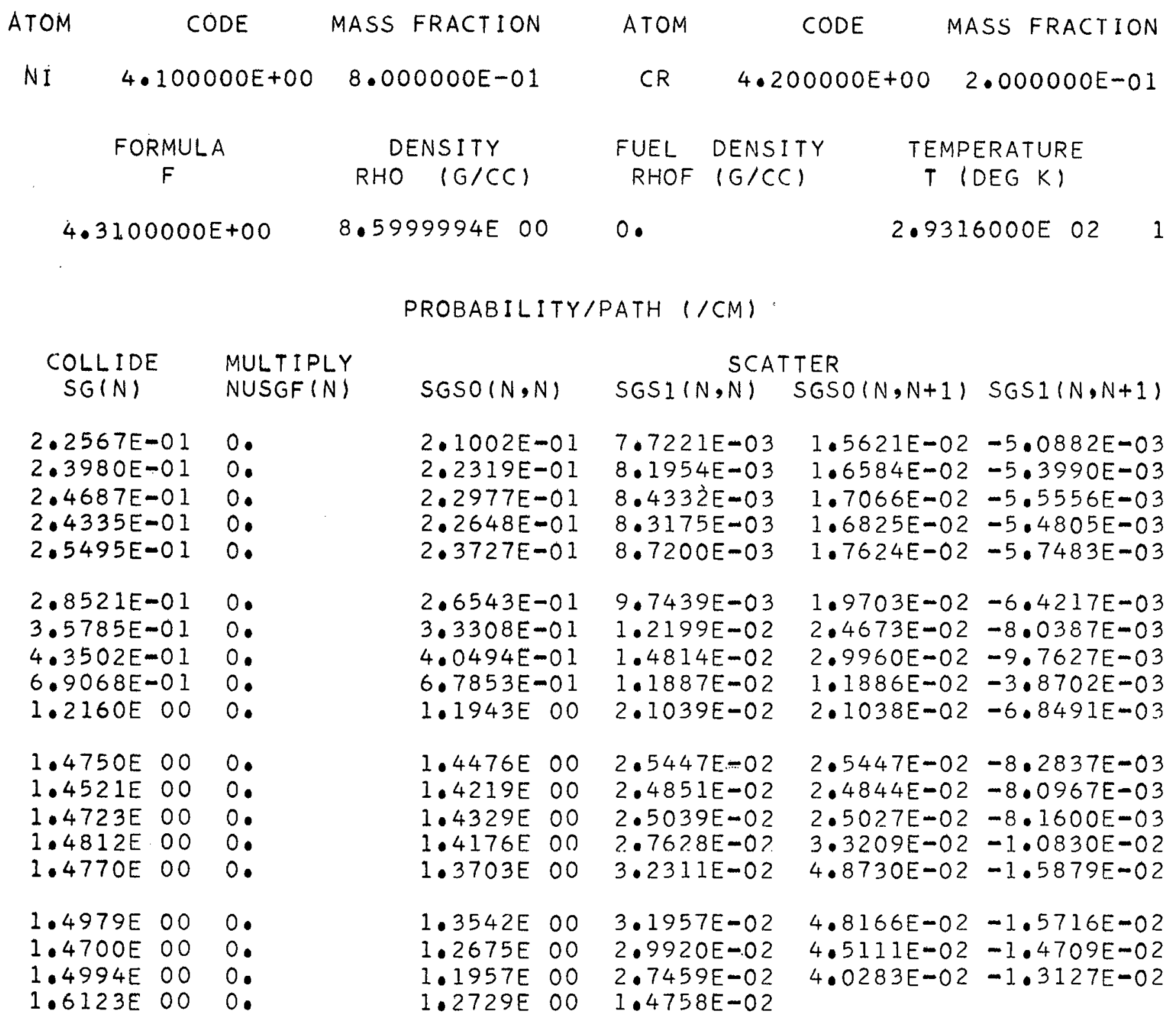




\begin{tabular}{|c|c|c|c|c|c|c|}
\hline ATOM & FORMULA & MASS FRACTION & ATOM & FO & & MASS FRACTION \\
\hline C & $3.3000000 \mathrm{E}+00$ & $8.8670000 \mathrm{E}-01$ & $\mathrm{H}$ & 2.040 & $E+\infty$ & $1.1330000 \mathrm{E}-\mathrm{OI}$ \\
\hline & $\begin{array}{c}\text { FORMULA } \\
\mathrm{F}\end{array}$ & $\begin{array}{c}\text { DENSITY } \\
\text { RHO }(G / C C)\end{array}$ & $\begin{array}{l}\text { FUEL } \\
\text { RHOF }\end{array}$ & $\begin{array}{l}\text { DENSITY } \\
(G / C C)\end{array}$ & $\begin{array}{c}\text { TEN } \\
\text { T }\end{array}$ & $\begin{array}{l}\text { ERATURE } \\
\text { DEG KI }\end{array}$ \\
\hline & & $9.2999951 E-01$ & 0. & & 2.93 & $6000 E \quad 02101$ \\
\hline
\end{tabular}

PROBABILITY/PATH $(/ C M)$

\begin{tabular}{|c|c|c|c|c|c|}
\hline COLLIDE & MULTIPLY & & 50 & $E R$ & \\
\hline$S G(N)$ & NUSGF (N) & $\operatorname{SGSO}(N, N)$ & $S G S I(N, N)$ & $S G S O(N, N+1)$ & SGSI $(N, N+1)$ \\
\hline $\begin{array}{l}.3135 E-01 \\
.6806 E-01 \\
.2923 E-01 \\
.9157 E-01 \\
.7523 E-01\end{array}$ & $\begin{array}{l}0 . \\
0 . \\
0 . \\
0 .\end{array}$ & $\begin{array}{l}5.5624 E-02 \\
6.4415 E-02 \\
8.4720 E-02 \\
1.0140 E-01 \\
1.2892 E-01\end{array}$ & $\begin{array}{l}2.3143 E-02 \\
3.0299 E-02 \\
4.1647 E-02 \\
5.3624 E-02 \\
6.9169 E-02\end{array}$ & $\begin{array}{l}4.1160 E-02 \\
5.2543 E-02 \\
7.1610 E-02 \\
9.0974 E-02 \\
1.1705 E-01\end{array}$ & $\begin{array}{l}1.2402 E-02 \\
2.0782 E-02 \\
3.0628 E-02 \\
4.3587 E-02 \\
5.7235 E-02\end{array}$ \\
\hline $\begin{array}{l}4.7603 E-01 \\
6.0020 E-01 \\
7.3599 E-01 \\
1.0626 E 00 \\
1.3737 E \quad 00\end{array}$ & $\begin{array}{l}0 . \\
0 . \\
0 . \\
0 .\end{array}$ & $\begin{array}{l}1.6223 E-01 \\
1.9819 E-01 \\
2.3468 E-01 \\
6.6812 E-01 \\
8.4834 E-01\end{array}$ & $\begin{array}{l}8.7886 E-02 \\
1.1146 E-01 \\
1.3753 E-01 \\
4.1488 E-01 \\
5.5271 E-01\end{array}$ & $\begin{array}{l}1.4847 E-01 \\
1.8709 E-01 \\
4.4060 E-01 \\
3.4305 E-01 \\
4.5628 E-01\end{array}$ & $\begin{array}{l}7.3575 E-02 \\
9.7396 E-02 \\
2.3857 E-01 \\
1.7213 E-01 \\
2.3229 E-01\end{array}$ \\
\hline $\begin{array}{ll}1.4399 E & 00 \\
1.4542 E & 00 \\
1.4547 E & 00 \\
1.4734 E & 00 \\
1.4959 E & 00\end{array}$ & $\begin{array}{l}0 . \\
0 . \\
0 . \\
0 . \\
0 .\end{array}$ & $\begin{array}{l}8.8588 E-01 \\
8.9392 E-C 1 \\
8.9392 E-01 \\
7.9042 E-01 \\
6.4316 E-01\end{array}$ & $\begin{array}{l}5.8284 E-01 \\
5.8930 E-01 \\
5.8902 E-01 \\
5.2930 E-01 \\
4.3442 E-01\end{array}$ & $\begin{array}{l}4.8100 E-01 \\
4.8630 E-01 \\
4.3849 E-01 \\
4.3833 E-01 \\
5.4880 E-01\end{array}$ & $\begin{array}{l}2.4557 E-01 \\
2.4841 E-01 \\
2.3310 E-01 \\
2.5647 E-01 \\
3.3108 E-01\end{array}$ \\
\hline $\begin{array}{ll}1.6522 E & 00 \\
2.087 C E & 00 \\
2.6928 E & 00 \\
3.0511 E & 00\end{array}$ & $\begin{array}{l}0 . \\
0 . \\
0 . \\
0 .\end{array}$ & $\begin{array}{l}7.0251 E-01 \\
8.7637 E-01 \\
1.2321 E 00 \\
3.0425 E 00\end{array}$ & $\begin{array}{l}4.7666 E-01 \\
6.0212 E-01 \\
7.8749 E-01 \\
8.6229 E-01\end{array}$ & $\begin{array}{l}6.1327 E-01 \\
8.2614 E-01 \\
1.4463 E \quad 00\end{array}$ & $\begin{array}{l}3.4213 E-01 \\
3.8488 E-01 \\
5.7858 E-02\end{array}$ \\
\hline
\end{tabular}


013

$1.3602 E-02$

019

$1.4881 E-03$

0115

$-6 \cdot 9070 E-08$

02.5

1.2195E-02

0211

$6.6392 E-05$

$0 \quad 217$

0.3

$6.3998 E-03$

0314

4. $0053 E-07$

046

$3.9031 E-02$

0412

4. $7469 E-05$

0418

0.511

$7.5302 E-04$

O 517

$3.2283 E-07$

0611

1.5881E-03

0617

$8.2583 E-08$

0712

4.6067E-04

0718

$1.0736 E-07$

0814

$1.5899 E-05$

0911

4.4447E-02

0917

$2.4612 E-06$

$0 \quad 1015$

1. $7829 \mathrm{E}-04$

01114

$7.6612 E-03$

ก $12 \quad 14$

5.725?E-0?

C. $13 \quad 15$

$7.68065-02$

01417

$5.6504 E-02$

$0 \quad 16 \quad 18$

$2.2 .557 E-01$
014

$98 E-03$

0110

$2.0140 E-04$

0116

0.

026

$7.3969 E-03$

0212

$9.0106 \mathrm{E}-06$

0218

0.

$8.5300 E-03$

0315

$7.2823 E-0.8$

047

$2.3673 E-02$

0413

$6.4910 E-06$

C 419

0.512

$1.0198 E-04$ O 518

$-2.5826 E-07$

0612

2.1488E-04

0618

0713

$6.2482 E-05$

0719

0

815

$2.7177 E-06$

0912

$6.0153 E-03$

0918

$8.9021 \mathrm{E}-\mathrm{C}_{7}$

01016

$6.5686 E-05$

0 1]. 15

$1.3909 E-03$

01215

1. $0.295 E-02$.

O 1316

$2.3255 E-02$

C 1418

$2.1435 E-02$

01619

$1.1053 \bar{E}-01$
015

$5.0038 E-03$

0111

$2.7274 E-005$

0117

0.7

4. $4864 E-03$

0213

$1.2508 E-06$

0219

0.

0310

$1.1545 \mathrm{E}-03$

0316

0.

048

1.4359 E-02

0414

$6.9370 E-07$

C 57

5. $0858 E-02$

0513

$1.3752 E-05$

O 519

0.

0613

$2.8904 E-05$

0619

0.

O 714

$7.4077 E-06$

$081 \mathrm{C}$

$5.2485 E-02$

0816

$9.5121 E-07$

0913

$8.1397 E-04$

C 919

4.1892E-07

$\begin{array}{lll}0 & 10 & 17\end{array}$

2.4180E-05

01116

$5.1153 E-0,4$

C $1 ? 16$

$3.8240 F-03$

O 1317

1.C395E-02

C 1419

1. $1449 E-02$

01719

$3.7588 E-01$
016

$3.0349 E-03$
0112

$3.6780 E-06$

- 118

0 .

$\begin{array}{lll}0 & 2\end{array}$

2.7212E-03

0214

$1.0210 E-07$

035

$2.8682 E-02$

0311

I. $5622 \mathrm{E}-04$

0317

0.

049

$1.9138 E-02$

0415

$1.4865 E-07$

058

$3.0847 E-02$

0514

$1.6787 E-06$

068

$6.5051 E-02$

0614

$3.7162 E-.06$

079

$1.8584 \mathrm{E}-01$

0715

$1.5030 E-06$

0811

$7.1031 \mathrm{E}-03$

0817

4.0766E-07

0914

$9.9023 E-05$

01012

5.9661E-02

01018

$9.0674 \mathrm{E}-06$

01117

$1.8825 \mathrm{E}-04$

01217

$1.4068 \mathrm{E}-03$

01318

$3.3432 E-03$

01517

1.902.8E-01 $\begin{array}{llllll}0 & 1 & 7 & 0 & 1 & 8\end{array}$

$1.8408 E-03 \quad 1.1165 E-03$

0113

5.0075E-07

0119

0 .

029

$3.6270 E-03$

0215

$5.1051 E-08$

036

$1.7396 E-02$

0312

$2.1192 E-05$

0318

0.

0410

$2.5901 E-03$

0416

$2.4775 E-07$

059

4.1115E-02

0515

$3.2283 E-07$

069

$8.6705 E-02$

0615

$6.6067 E-07$

0710

2.5151E-02

0716

$5.3679 E-07$

0812

$9.6120 E-04$

0818

$6.7943 E-07$

0915

$1.8014 E-05$

01013

$8.0742 E-03$

01019

4.7797E-06

01118

$7.148,4 E-05$

01218

$5.3349 E-04$

01319

$2.1059 E-03$

01518

$7.2184 \mathrm{E}-02$

0114

1.2087E-07

024

2.0107E-02

0210

4.9085E-04

0216

0

037

$1.0551 E-02$

0313

$2.7673 E-06$

0319

0.

0411

$3.5047 E-04$ 0417

$-1.4865 E-07$

0510

$5.5644 E-03$

0516

$6.4565 E-08$

0610

1. $1734 \mathrm{E}-02$

0616

$2.4775 E-07$

0711

$3.4038 E-03$

0717

0 .

0813

$1.3025 E-04$

0819 
113

$8.4734 E-03$

119

$1.7692 E-04$

1115

$3.0467 E-07$

125

5.9217E-03

1211

$3.1684 E-06$

1217

0.

138

$1.8899 E-03$

1314

$1.2147 E-07$

146

$2.4307 E-02$

1412

$2.0164 E-06$

1418

0

1511

$3.7709 E-05$

1517

$-1.7498 E-07$

1611

$9.5499 E-05$

$\begin{array}{lll}1 & 6 & 17\end{array}$

$5.5091 E-08$

1712

1.847 SE-05

1718

$2.8649 E-07$

1814

$1.3598 E-06$

1911

$8.8567 E-03$

1917

4.5413E-07

11015

$7.0336 E-06$

1. 1114

6.1.9935-04

11214

$1.1943 E-02$

$113 \quad 15$

$2.1651 E-02$.

$114 \quad 17$

$1.3075 E-02$

11618

5.? $380 E-0$ ? $\begin{array}{lll}1 & 1 & 4\end{array}$

$4.0062 E-03$

1110

$1.1236 \mathrm{E}-05$

1116

0 .

126

$2.8012 E-03$

1212

$7.8359 E-07$

1218

0.

139

$1.6 ? .02 E-03$

$\begin{array}{lll}1 & 3 & 15\end{array}$

$4.8589 E-08$

147

$1.1488 E-02$

1413

$4.9584 E-07$

1419

0.

1512

$3.8979 E-06$

1518

$3.0419 E-07$

1612

$7.9330 E-06$

$\begin{array}{lll}1 & 6 & 18\end{array}$

$1.6527 E-07$

1713

$3.1156 E-06$

1719

0 .

1815

$3.6262 E-07$

1912

$4.6742 E-04$

1918

$3.1439 E-C 7$

11016

$3.4230 E-06$

11115

$5.6332 E-05$

11215

1.1193E-03

11316

$4.7784 E-C 3$

11418

2. $9837 E-.3$

11619

$-2 . ? 449 E-02$
115

$1.8953 \mathrm{E}-03$

1111

$1.4578 \mathrm{E}-06$

1117

c.

$\begin{array}{lll}1 & 2 & 7\end{array}$

$1 \cdot 3263 E-03$

1213

$2.3849 E-07$

1219

0.

1310

8.7071E-05

1316

$1.2147 E-07$

148

$5.43 ? 9 E-03$

1414

$2.3139 E-07$

157

$3.1672 E-02$

1513

$8.6142 E-07$

1519

0.

1613

$1.652 .7 \mathrm{E}-06$

1619

0.

7.1622 E-07

$18 \quad 10$

$1.2723 E-02$

1.816

$1.8131 E-07$

1913

$3.3011 E-05$

1919

$5.9386 E-07$

11017

$1.6412 E-06$

11116

$1.9758 E-05$

11216

$2.63315-04$

11317

$\therefore .03445-03$

$114 \quad 19$

$7.7141 E-04$

11719

$-5.5947 E-0 ?$
116

$8.9749 E-04$

1112

$4.1487 E-07$

1118

0.

$\begin{array}{lll}1 & 2 & 8\end{array}$

$6.2894 E-04$

1214

$6.8139 E-08$

135

$1.7864 \mathrm{E}-02$

1311

$6.7053 E-06$

I 317

0 .

149

$4.6388 E-03$

1415

$3.3056 E-08$

158

$1.4969 E-02$

1514

$2.5843 E-07$

168

4.0510E-02

1614

$3.3054 E-07$

179

$9.4897 E-02$

1715

$5.0136 E-07$

1811

$6.5919 E-04$

1817

$2.7196 E-07$

1914

4.4889E-06

11012

1.1890 E-02

11018

$1.0785 E-06$

11117

$7.4775 E-06$

$\because 1217$

$5.6986=-05$

11313

$2.1573 E-04$

$\begin{array}{lll}1 & 15 & 17\end{array}$

$7.1330 E-02$

$\begin{array}{llllll}1 & 1 & 7 & 1 & 1 & 8\end{array}$

4.2568E-04 2.0243E-04

$\begin{array}{llllll}1 & 1 & 13 & 1 & 1 & 14\end{array}$

$1 \cdot 3829 E-07-1.8943 E-07$

1119

124

0.

129

$5.4352 E-04$

1215

$1.1924 E-07$

136

$8.4443 E-03$

1312

$1.1904 E-06$

1318

0

1410

$2.402 .0 E-04$

1416

$-2 \cdot 1899 E-07$

159

1.2762E-02

1515

$8.6142 E-08$

169

$3.4508 \mathrm{E}-02$

1615

$2.2036 E-07$

1710

4. 768 ? E-03

1716

$7.162 .2 E-08$

1812

4.2335E-05

1818

$-6 \cdot 1758 E-07$

$1 \bigcirc 15$

$1.2925 E-06$

11013

$6.2803 E-04$

11019

$1.8287 E-06$

11118

$3.5645 E-06$

11 ? 18

$2.0459 F-05$

11319

$\therefore .8677=-05$

11518

$1 \cdot 2525 E-02$

1210

$3 \cdot 1429 E-05$

1216

0 .

137

$3.9934 E-03$

1313

$3.6442 E-07$

1319

0.

1411

$1.5404 E-05$

1417

$3.8427 E-07$

1510

$6.4993 E-04$

1516

$8.6142 E-08$

1610

$1.7418 E-03$

1616

$5.5091 \mathrm{E}-0$ ?

1711

$2.53425-04$

1717

$7 \cdot 162.2 E-08$

1813

5.5752E-06 


\begin{tabular}{|c|c|c|c|c|c|c|}
\hline ATOM & CODE & MASS FRACTION & ATOM & \multicolumn{2}{|c|}{ CODE } & MASS FRACTION \\
\hline $\begin{array}{l}C \\
H\end{array}$ & $\begin{array}{l}3.300000 E+00 \\
2.040000 E+00\end{array}$ & $\begin{array}{l}5.870000 E-01 \\
8.150000 E-02\end{array}$ & 0 & \multicolumn{2}{|c|}{$3.600000 E+00$} & $3.315000 E-01$ \\
\hline & $\begin{array}{c}\text { FORMULA } \\
F\end{array}$ & $\begin{array}{cc}\text { DENSITY } \\
\text { RHO }(G / C C)\end{array}$ & $\begin{array}{l}\text { FUEL } \\
\text { RHOF }\end{array}$ & $\begin{array}{l}\text { DENSI TY } \\
(G / C C)\end{array}$ & \multicolumn{2}{|c|}{ TEMPERATURE } \\
\hline 2 & $500000 E+00$ & $1.1799994 \mathrm{E} \quad 00$ & 0. & & $2 \cdot 9$ & $316000 \mathrm{E} \quad 02101$ \\
\hline
\end{tabular}

PROBABILITY/PATH (/CM)

\begin{tabular}{|c|c|c|c|c|c|}
\hline \multirow{2}{*}{$\begin{array}{c}\text { COLLIDE } \\
\text { SG }(N)\end{array}$} & MULTIPLY & \multicolumn{4}{|c|}{ SCATTER } \\
\hline & NUSGF $(N)$ & $\operatorname{SGSO}(N, N)$ & $\operatorname{SGSI}(N, N)$ & $\operatorname{SGSO}(N, N+1)$ & $\operatorname{SGS} 1(N, N+1)$ \\
\hline $\begin{array}{l}1.2561 E-01 \\
1.7325 E-01 \\
2.2824 E-01 \\
2.8771 E-01 \\
3.9400 E-01\end{array}$ & $\begin{array}{l}0 . \\
0 . \\
0 . \\
0 .\end{array}$ & $\begin{array}{l}5.5438 E-02 \\
7.4189 E-02 \\
9.2176 E-02 \\
1.0938 E-01 \\
1.5731 E-01\end{array}$ & $\begin{array}{l}2.1639 E-02 \\
2.9800 E-02 \\
4.0023 E-02 \\
5.1232 E-02 \\
6.8832 E-02\end{array}$ & $\begin{array}{l}3.8625 E-02 \\
5.2418 E-02 \\
6.9534 E-02 \\
8.7787 E-02 \\
1.1871 E-01\end{array}$ & $\begin{array}{l}1.0984 E-02 \\
1.7594 E-02 \\
2.6664 E-02 \\
3.8314 E-02 \\
4.8595 E-02\end{array}$ \\
\hline $\begin{array}{l}4.9403 E-01 \\
5.9396 E-01 \\
7.1488 E-01 \\
1.0116 E \quad 00 \\
1.2948 E \quad 00\end{array}$ & $\begin{array}{l}0 . \\
0 . \\
0 . \\
0 . \\
0 .\end{array}$ & $\begin{array}{l}1.9394 E-01 \\
2.1672 E-01 \\
2.4783 E-01 \\
6.4935 E-01 \\
8.1315 E-01\end{array}$ & $\begin{array}{l}8.6781 E-02 \\
1.0670 E-01 \\
1.3010 E-01 \\
3.8094 E-01 \\
5.0667 E-01\end{array}$ & $\begin{array}{l}1.4919 E-01 \\
1.8108 E-01 \\
4.1165 E-01 \\
3.1537 E-01 \\
4.1866 E-01\end{array}$ & $\begin{array}{l}6.2953 E-02 \\
8.571,2 E-02 \\
2.1480 E-01 \\
1.5640 E-0.1 \\
2.1132 E-01\end{array}$ \\
\hline $\begin{array}{ll}1.3552 E & 00 \\
1.3683 \mathrm{E} & 00 \\
1.3688 \mathrm{E} & 00 \\
1.3857 \mathrm{E} & 00 \\
1.4061 \mathrm{E} & 00\end{array}$ & $\begin{array}{l}0 . \\
0 . \\
0 . \\
0 . \\
0 .\end{array}$ & $\begin{array}{l}8.4741 E-01 \\
8.5475 E-01 \\
8.5475 E-01 \\
7.5941 E-01 \\
6.234 I E-01\end{array}$ & $\begin{array}{l}5.3417 E-01 \\
5.4006 E-01 \\
5.3981 E-01 \\
4.8552 E-01 \\
3.9936 E-01\end{array}$ & $\begin{array}{l}4.4122 E-01 \\
4.4606 E-01 \\
4.0242 E-01 \\
4.0300 E-01 \\
5.0527 E-01\end{array}$ & $\begin{array}{l}2.2345 E-01 \\
2.2604 E-01 \\
2.1206 E-01 \\
2.3317 E-01 \\
3.0082 E-01\end{array}$ \\
\hline $\begin{array}{ll}1.5487 E & 00 \\
1.9459 E & 00 \\
2.5015 E & 00 \\
2.8411 E & 00\end{array}$ & $\begin{array}{l}0 . \\
0 . \\
0 .\end{array}$ & $\begin{array}{l}6.7759 E-01 \\
8.3659 E-01 \\
1.1639 E \quad 00 \\
2.8241 E \quad 00\end{array}$ & $\begin{array}{l}4.3791 E-01 \\
5.5245 E-01 \\
7.2177 E-01 \\
7.8880 E-01\end{array}$ & $\begin{array}{l}5.6411 E-01 \\
7.5844 E-01 \\
1.3245 E 00\end{array}$ & $\begin{array}{l}3.1091 E-01 \\
3.4990 E-01 \\
5.1416 E-02\end{array}$ \\
\hline
\end{tabular}


013

$1.2414 E-02$ 019

$1.3582 E-03$

0115

$-6.3040 E-08$

025

1. II $31 E-02$

011
011

$6.0596 E-05$

0217

0 .

038

$5.8410 E-03$

0314

$3.6556 E-07$

046

$3.5623 E-02$

0412

4. $3325 E-05$

O. 418

0 .

$0 \quad 511$

$6.8728 E-04$

$0 \quad 517$

$2.9464 E-07$

0611

$1.4494 E-03$

$\begin{array}{lll}0 & 6 & 17\end{array}$

$7.5373 E-08$

0712

4. $2046 E-04$

0718

$9.7986 E-08$

0814

$1.4511 E-05$

0911

4.0566E-02

0
017

2. $2463 E-06$

$0 \quad 1015$

$1.6272 \mathrm{E}-04$

01114

$6.9923 E-03$

01214

$5.2254 E-02$

01315

7.0101E-02

01417

5.1571E-02

01618

$2.0141 E-01$
014

$7 \cdot 5296 E-03$

0110

1. $8382 \mathrm{E}-04$

0116

026

$6.7511 E-03$

0212

$8 \cdot 2239 E-06$

0218

0.

039

$7.7854 \mathrm{E}-03$

0315

$6.6466 E-08$

047

2.1607E-02

0413

$5.9244 E-06$

0419

0 .

0512

9.3077E-05

0518

$-2.3571 E-07$

0612

$1.9612 E-04$

0618

0 .

0713

$5.7028 E-05$

0719

0.

0815

$2.4805 E-06$

0912

$5.4901 E-03$

0918

$8.1249 \cdot E-07$

01016

$5.995 I E-05$

01115

$1.2695 E-03$

01215

$9.4871 E-03$

01316

$2.5789 E-02$

01418

$1.9564 \mathrm{E}-02$

01619

$1.0088 \mathrm{E}-01$
0115

0111

$2.4893 E-05$

$0 \quad 1 \cdot 17$

027

4.0944 E-03

0213

$1.1416 E-06$

0219

0.

0310

$1.0537 E-03$

O 316

048

1. $3105 E-02$

0414

$6.3314 E-07$

057

4.6418E-02

0513

1.2552E-05

O 519

0613

$2.6381 E-05$

0619

0 .

0714

$6.7610 E-06$

0810

4.7903E-02

0816

$8.6817 E-07$

0913

$7.4290 E-04$

0919

$3.8235 E-07$

$0 \quad 1017$

$2.2069 E-05$

01116

4. $6687 E-04$

01216

$3.4902 E-03$

$0 \quad 1317$

$9.4871 \mathrm{E}-03$

01419

$1.0450 E-02$

01719

$3.4306 E-01$ $\begin{array}{lllllllll}0 & 1 & 6 & 0 & 1 & 7 & 0 & 1 & 8\end{array}$

$2.7700 E-03$

0112

$3.3569 E-06$

0118

0 .

028

$2.4836 E-03$

0214

$9.3189 E-08$

035

$2.6178 E-02$

0311

1.4259E-04

$0 \quad 317$

0.

049

$1.7467 E-02$

0415

$1.3567 E-07$

058

$2.8154 E-02$

0514

$1.5321 E-06$

068

$5.9372 E-02$

0614

$3.3918 E-06$

079

$1.6961 E-01$

0715

$1.3718 E-06$

0811

$6.4830 E-03$

0817

$3.7207 E-07$

0914

$9.0378 E-05$

01012

$5.4452 E-02$

01018

8. $2757 E-06$

01117

1. $7182 \mathrm{E}-04$

$0 \quad 1217$

$1.2840 E-03$

$0 \quad 1318$

$3.5989 E-03$

$0 \quad 1517$

$1.7367 \mathrm{E}-01$

$1.0190 E-03$

0113

4.5704E-07

0114

0119

$1.1032 E-07$

0.

029

$3.3103 E-03$

0215

4. $6595 E-08$

036

$1.5878 E-02$

0312

I. $9342 E-05$

0318

0 .

0410

024

$1.8351 E-02$

0210

$4.4799 E-04$

0216

0 .

$\begin{array}{lll}0 & 3\end{array}$

$9.6303 E-03$

0313

$2.5257 E-06$

0319

0.

$\begin{array}{llll}0 & 4 & 11\end{array}$

$2.3640 E-03 \quad 3.1987 E-04$

$\begin{array}{llllll}0 & 416 & 0 & 4 & 17\end{array}$

$2.2612 E-07-1.3567 E-07$

0590510

$3.7526 E-02 \quad 5.0786 E-03$

051500516

$2.9464 E-07 \quad 5.8928 E-08$

069

0610

$7.9136 E-02 \quad 1.0710 E-02$

0615

$6.0299 E-07$

0710

$2.2955 E-02$

0716

4.8993E-07

0812

$8.7728 E-04$

0818

$6.2012 E-07$

0616

$2.2612 E-07$

0711

$3.1067 E-03$

0717

0915

$1.6441 \mathrm{E}-05$

01013

$7 \cdot 3693 E-03$

$0 \quad 1019$

4.3624E-06

01118

$6.5244 E-05$

01218

4.8691E-04

01319

$1.9221 E-03$

01518

0813

I. $1888 \mathrm{E}-04$

0819

$-3.7207 E-07$

0916

$6.0220 E-06$

01014

8.9593E-04

01113

5.7505E-02

01119

$0 \quad 1219$

$3.4756 E-05$ 
$\begin{array}{llllll}1 & 1 & 3 & 1 & 1 & 4\end{array}$

$7.7336 E-03 \quad 3.6565 E-03$ 119

$1.6148 E-04$ 1115

$2.7807 E-07$

125

$5.4047 E-03$

1211

$2.8918 E-06$

$\begin{array}{lll}1 & 2 & 17\end{array}$

c.

138

$1.7249 \mathrm{~F}-03$

1314

$1.1087 \mathrm{E}-07$

146

$2.2185 E-02$

1412

$1.8404 E-06$

1418

0.

1511

$3.4417 E-05$

1517

$-1.5970 E-07$

1611

$8.7162 E-05$

1617

5.0281E-08

1712

$1.6865 \mathrm{E}-05$

1718

$2.6148 E-07$

1814

$1.2411 E-06$

1911

$8.0835 E-03$

1917

4. $1448 E-07$

11015

$6.4195 E-06$

11114

$5.6581 E-04$

11214

$1.0900 E-02$

11315

$1.9761 E-02$

$114 \quad 17$

$1.1933 E-02$

$116 \quad 18$

$4.7807 E-02-2.0490 E-02$
$1.72 \frac{1}{5}$

$\begin{array}{llll}1 & 1 & 11\end{array}$

$1.3305 E-06$

1117

0.

127

126

$2.5567 E-03$

1212

$7.1518 E-07$

1218

0.

139

$1.4787 E-03$

1315

4. $4347 E-08$

147

$1.0485 \mathrm{E}-02$

1413

4. $5255 E-07$

1419

0

1512

$3.5576 E-06$

1518

$2.7763 E-07$

1612

$7.2405 E-06$

1618

$1.5084 \mathrm{E}-07$

1713

$2.8436 E-06$

1719

1815

$3.3096 E-07$

1912

4.2661E-04

1918

$2.8695 E-07$

$110 \quad 16$

$3.1242 E-06$

11115

$6.0541 E-05$

11215

$1.0216 \mathrm{E}-03$

11316

4. $3613 E-03$

11418

$2.7232 E-03$

11619

1613

$1.5084 \mathrm{E}-06$

1619

0.

1714

$6.5369 E-07$

1810

$1.1612 E-02$

1816

$1.6548 E-07$

$3.0129 E-05$

1919

5.4201E-07

$110 \quad 17$

$1.4979 E-06$

11116

I. $8033 E-05$

11216

$2.4032 E-04$

11317

$9.4406 E-04$

11419

$7.0406 E-04$ $11 / 19$

$-6.0098 E-02$
116

$8.1913 E-04$

1112

$3.7865 E-07$

1118

0.

128

$5.7403 E-04$

1214

$6.2190 E-08$

135

$1.6304 \mathrm{E}-02$

1311

6.1199E-06

1317

0.

149

4. $2338 E-03$

1415

$3.0170 E-08$ 158

$1.3662 E-02$

1514

$2.3586 E-07$

168

$3.6974 E-02$

1614

1913

11318

11517

$6.5102 E-02$

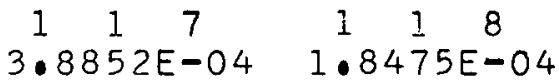
$\begin{array}{llllll}1 & 1 & 13 & 1 & 1 & 14\end{array}$

$1.2622 E-07-1.7289 E-07$

$\begin{array}{llllll}1 & 1 & 19 & 1 & 2 & 4\end{array}$

$0.1 .1432 E-0$ ?

1291210

4.9607E-04 2.8685E-05

$1215 \quad 12216$

$1.0883 E-07$

$\begin{array}{lllll}1 & 3 & 6 & 1 & 3\end{array}$

$7.7070 E-03 \quad 3.6447 E-03$

$\begin{array}{llllll}1 & 3 & 12 & 1 & 3 & 13\end{array}$

$1.0865 E-06 \quad 3.3260 E-07$

$\begin{array}{llllll}1 & 318 & 1 & 319\end{array}$

0.0 .

$1410 \quad 1411$

$2.1923 E-04 \quad 1.4059 E-05$

$\begin{array}{llllll}14 & 4 & 16 & 4 & 17\end{array}$

$3.5073 E-07$

1510

159

$1.1648 \mathrm{E}-02$

1515

$7.8621 E-08$

169

$3 \cdot 1495 E-02$

1615

$2.0112 E-07$

1710

4. $3520 E-03$

.1716

$6.5369 E-08$

1812

$3.8639 E-05$

1818

$\begin{array}{lll}1 & 8 & 17\end{array}$

$5.6366 E-07$

1915

1.1797E-06

$110 \quad 13$

$5.7320 E-04$

$110 \quad 19$

1.6691 E-06

$\begin{array}{lll}1 & 11 & 18\end{array}$

$3.3446 E-06$

11218

$1.8673 E-05$

11319

$1.7046 \mathrm{E}-05$

$5.9319 E-04$

1516

$7.8621 E-08$

1610

$1.5897 E-03$

1616

$5.0281 \mathrm{E}-08$

1711

$2.3129 E-04$

1717

$6.5369 E-08$

1813

$5.0885 \mathrm{E}-06$

1819

$8.9462 E-07$

1916

$7.0143 E-07$

$110 \quad 14$

$3.6720 E-05$

11113

1. $1461 \mathrm{E}-02$

11119

4.3389E-06

11219

$1.4010 E-05$

11416

11518

$.3481 E-02$

$\begin{array}{ccc}11518 & 11519 \\ 1.2163 E-02 & -1.3107 E-03\end{array}$ 


\begin{tabular}{|c|c|c|c|c|c|}
\hline ATOM & CODE & MASS FRACTION & ATOM & CODE & MASS FRACTION \\
\hline C & $3 \cdot 300000 E+00$ & $8.563000 E-01$ & $H$ & $2.040000 E+00$ & $1.437000 \mathrm{E}-01$ \\
\hline & $\begin{array}{c}\text { FORMULA } \\
\text { F }\end{array}$ & $\begin{array}{c}\text { DENSITY } \\
\text { RHO }(G / C C)\end{array}$ & $\begin{array}{l}\text { FUEL } \\
\text { RHOF }\end{array}$ & $\begin{array}{l}\text { DENSITY } \\
(G / C C)\end{array}$ & $\begin{array}{c}\text { TEMPERATURE } \\
T(D E G \quad K)\end{array}$ \\
\hline & $700000 E+00$ & $9.2000104 E-01$ & 0. & 2. & $316000 \mathrm{E} \quad 02 \quad 10$ \\
\hline
\end{tabular}

PROBABILITY/PATH (/CM)

\begin{tabular}{|c|c|}
\hline $\begin{array}{l}\text { COLLIDE } \\
\text { SG (N) }\end{array}$ & $\begin{array}{l}\text { MULTIPLY } \\
\text { NUSGF }(N)\end{array}$ \\
\hline $\begin{array}{l}1.4717 E-01 \\
1.9260 E-01 \\
2.6471 E-01 \\
3.4076 E-01 \\
4.3953 E-01\end{array}$ & $\begin{array}{l}0 . \\
0 . \\
0 . \\
0 . \\
0 .\end{array}$ \\
\hline $\begin{array}{l}5.5845 E-01 \\
7.0819 E-01 \\
8.7373 E-01 \\
1.2781 E 00 \\
1.6653 E 00\end{array}$ & $\begin{array}{l}0 . \\
0 . \\
0 . \\
0 . \\
0 .\end{array}$ \\
\hline $\begin{array}{ll}1.7484 E & 00 \\
1.7664 E & 00 \\
1.7670 E & 00 \\
1.7898 E & 00 \\
1.8174 E & 00\end{array}$ & $\begin{array}{l}0 . \\
0 . \\
0 . \\
0 . \\
0 .\end{array}$ \\
\hline $\begin{array}{ll}2.0136 E & 00 \\
2.5591 \mathrm{E} & 00 \\
3.3187 \mathrm{E} & 00 \\
3.7827 \mathrm{E} & 00\end{array}$ & $\begin{array}{l}0 . \\
0 . \\
0 .\end{array}$ \\
\hline
\end{tabular}

\begin{tabular}{|c|c|c|c|}
\hline \multicolumn{4}{|c|}{ SCATTER } \\
\hline,$N)$ & $\operatorname{SGS} 1(N, N)$ & G501 & $(, N+1)$ \\
\hline $\begin{array}{l}59 E-02 \\
66 E-02 \\
76 E-02 \\
12 E-01 \\
43 E-01\end{array}$ & $\begin{array}{l}2 \cdot 6 \\
3 \cdot 5 \\
4 \cdot 8 \\
6 \cdot 3\end{array}$ & $\begin{array}{l}4.6 \\
6.0 \\
8.2 \\
1.06 \\
1.36\end{array}$ & $\begin{array}{l}E-02 \\
E-02 \\
E=02 \\
E-02 \\
E-02\end{array}$ \\
\hline $\begin{array}{l}1.7707 E-01 \\
2.1805 E-01 \\
2.6055 E-01 \\
7.8756 E-01 \\
1.0108 E \quad 00\end{array}$ & $\begin{array}{l}1.0446 E-01 \\
1.3313 E-01 \\
1.6511 E-01 \\
5.1617 E-01 \\
6.8885 E-01\end{array}$ & $\begin{array}{l}4 E-01 \\
7 E-01 \\
1 E-01 \\
3 E-01 \\
6 E-01\end{array}$ & $\begin{array}{l}2 E-02 \\
1 E-01 \\
8 E-01 \\
6 E-01 \\
1 E-01\end{array}$ \\
\hline $\begin{array}{l}1.0579 E \quad 00 \\
1.0680 E \quad 00 \\
1.0680 E \quad 00 \\
9.3915 E-01 \\
7.5698 E-01\end{array}$ & $\begin{array}{l}E-01 \\
E-01 \\
E-01 \\
E-01 \\
E-01\end{array}$ & $\begin{array}{l}5.9888 E-01 \\
6.0553 E-01 \\
5.4554 E-01 \\
5.4373 E-01 \\
6.7912 E-01\end{array}$ & $\begin{array}{l}48 E-01 \\
34 E-01 \\
83 E-01 \\
63 E-01 \\
19 E-01\end{array}$ \\
\hline 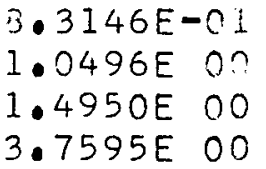 & $\begin{array}{l}5.294-01 \\
7.9355-n \\
9.8203 \mathrm{E}-01 \\
1.0787 \mathrm{~F} \text { on }\end{array}$ & $\begin{array}{l}.6001 E-0 \\
.0271 \% \text { OO } \\
1.8056 \mathrm{E} 00\end{array}$ & $\begin{array}{l}405=01 \\
\because \because 558=01 \\
7.5256 F-02\end{array}$ \\
\hline
\end{tabular}


013

$1.7066 E-02$

019

$1.8671 E-03$

0115

$-8.6660 E-08$

0
$1.5301 E-02$

0211

8. $3301 E-05$

0217

0.38

$8.0296 E-03$

0314

$5.0253 E-07$

0
$4.8971 E-02$

0412

$5.9558 E-05$

0418

0.511

$9.4480 E-04$

$0 \quad 517$

$4.0504 \mathrm{E}-07$

0611

$1.9925 E-03$ 0617

$1.0362 E-07$ 0712

$5.7800 E-04$

0718

$1.3470 E-07$

0814

$1.9948 E-05$

0911

5.5766E-02 0
017

3.0880E-06 01015

$2.2370 E-04$ 01114

$9.6123 E-03$ 01214

$7.1834 \mathrm{E}-02$ $0 \quad 1315$

$9.6367 E-02$ $0 \quad 1417$

$7.0895 E-02$ 01618

$2.7687 E-01$
0414

0110

$2.5269 E-04$

0116

0 .

026

$9.2807 E-03$

0212

$1.1305 E-05$

0218

0.39

$1.0702 E-02$

0315

$9.1370 E-08$

047

$2.9702 E-02$

0413

$8.1442 E-06$

0419

0 .

$0 \quad 512$

1.2795E-04

0518

$-3.2403 E-07$

0612

$2.6961 E-04$ 0618

0 .

0713

$7.8395 E-05$

0719

0.

0815

$3.4099 E-06$ 0912

$7.5472 E-03$

0918

1.1169E-06

01016

$8.2414 E-05$

01115

$1.7452 E-03$

01215

$1.3042 E-02$ 01316

3.5451E-02 01418

$2.6894 E-02$ 01619

I. $3868 \mathrm{E}-01$ $\begin{array}{lll}0 & 1 & 5\end{array}$

$6.2781 E-03$

. $4220 E-05$

0117

0.

$\begin{array}{lll}0 & 2\end{array}$

$5.6290 E-03$

0213

$1.5693 \mathrm{E}-06$

0219

0.310

1. $4485 E-03$

0316

0.

048

1. $8015 E-02$

0414

$8.7037 E-07$

057

$6.3811 E-02$

0513

1. $7255 \mathrm{E}-05$

$0 \quad 519$

0.

0613

$3.6265 E-05$

0619

0.

0714

$9.2943 E-06$

0810

$6.5852 E-02$

0816

$1.1935 E-06$

0913

$1.0213 E-03$

0919

5.2561E-07

01017

$3.0338 E-05$

01116

$6.4180 E-04$

01216

4.7979E-03

$\begin{array}{lll}0 & 13 & 17\end{array}$

$1.3042 E-02$

01419

$1.4365 E-02$

01719

$4.7161 E-01$ $\begin{array}{lllllllll}0 & 1 & 6 & 0 & 1 & 7 & 0 & 1 & 8\end{array}$

$3.8079 E-03$

0112

4.6147E-06

0118

0.

028

$3.4142 E-03$

0214

I.2811E-07

035

$3.5986 \mathrm{E}-02$

0311

$1.9601 E-04$

0317

0.

049

$2.4012 E-02$

0415

I.8651E-07

058

$3.8703 E-02$

$0 \quad 514$

$2.1062 E-06$

068

$8 \cdot 1619 E-02$

0614

4.6627E-06

079

$2.3317 E-01$

0715

$1.8858 E-06$

0811

8.9121E-03

0817

5.1148E-07

0914

1. $2424 E-04$

01012

$7.4855 E-02$

01018

$1.1377 E-05$

$0 \quad 1117$

2. $3619 E-04$

01217

$1.7650 E-03$

01318

4. $9474 E-03$

01517

$2.3874 E-01$
$2.3096 E-03 \quad 1.4008 E-03$

0113

$6.2829 E-07$

0119

0.

029

4.5507E-03

0215

$6 \cdot 4053 E-08$

036

$2.1827 \mathrm{E}-02$

0312

$2.6589 E-05$

0318

0.

0410

$3.2497 E-03$

$\begin{array}{cccc}0 & 416 & 0 & 417 \\ 3.1085 \mathrm{E}-07 & -1.8651 \mathrm{E}-07\end{array}$

$\begin{array}{llllll}0 & 5 & 9 & 5 & 10\end{array}$

5.1587E-02 6.9815E-03

$0 \quad 515$

4.0504E-07

069

0516

$8 \cdot 1008 \mathrm{E}-08$

$1.0879 E-01$

0615

$8.2892 E-07$

0710

$3.1556 E-02$

0716

$6.7350 E-07$

0812

$1.2060 E-03$

0818

$8.5247 E-07$

0915

$2.2601 E-05$

01013

$1.0131 E-02$

01019

5.9970E-06

01118

$8.9690 E-05$

01218

$6.6936 E-04$

01319

0610

$1.4723 E-02$

0616

3.1085E-07

0711

4.2707E-03

0717

0.

0813

$1.6342 E-04$

0819

$-5.1148 E-07$

0916

$8.2784 E-06$

01014

$1.2316 \mathrm{E}-03$

01113

7.9052E-02

$0 \quad 1119$

4. $7779 E-05$

01219

$3.5766 E-04$

$2.6423 E-03$

01518

01416

$1.9271 E-01$

01519

$9.0568 E-02$

$4.8020 E-02$ 
$10031 E^{3}-02$ 199 1115

3.8226E-07 125

$7.4298 \mathrm{E}-03$

1211

$3.9754 \mathrm{E}-06$

1217

0.

138

2. $3712 E-03$ 1314

$1.5241 E-07$ 146

$3.0498 E-02$

1412

2.5299E-06

1418

0.

$\therefore \quad 511$

4.7312E-05 1517

$-2.1954 E-07$ 1611

$1.1982 E-04$ $\begin{array}{lll}1 & 6 & 17\end{array}$

$6.9121 E-08$

1712

$2.3185 E-05$

1718

3.5945E-07

1814

$1.7061 \mathrm{E}-06$ 1911

1.1112E-02 $\begin{array}{lll}1 & 9 & 17\end{array}$

5.6978E-07 11015

8.8249E-06 11114

$7.7781 \mathrm{E}-04$ 11214

$1.4984 \mathrm{E}-02$ 11315

2.7165E-02 11417

$1,6404 \mathrm{~F}-02$ 11618

$6.5720 E-02$
114 1110

$1.4098 E-05$

1116

126

$3.5146 E-03$

1212

$9.8316 E-07$

. 1218

0.

139

2.0328E-03 1315

$6.0964 E-08$ 147

$1.4414 E-02$

1413

$6.2212 E-07$

1419

0 .

1512

4.8906E-06

1518

$3.8166 E-07$

1612

$9.9534 E-06$

1618

2.0736 E-0 7

1713

3.9090E-06

1719

0 .

1815

4.5497E-07

1912

$5.8646 E-04$

1918

$3.9446 E-07$

11016

4. $2948 E-06$

11115

8.3225E-05

11215

$1.4043 E-03$

11316

5.9954E-03

11418

$3.7436 E-03$

11619

$2.8167 E-02$
$2 \stackrel{1}{1} \stackrel{5}{3779 E-03}$

1111

I.8291E-06

1117

0.

127

$1.6641 \mathrm{E}-03$

1213

2.9922E-07

1219

0.

1310

$1.0925 E-04$ 1316

1.5241E-07

148

6.8153E-03

1414

2.9032E-07

157

3.9738E-02

1513

$1.0808 E-06$

1519

0.

1613

2.0736E-06

1619

0.

1714

8.9863E-07

1810

$1.5963 \mathrm{E}-02$

1816

2. $2748 E-07$ 1913

4.1419E-05

1919

$7.4510 E-07$

$110 \quad 17$

2.0591E-06

11116

2.4790E-05

11216

$3.3037 E-04$

$\begin{array}{lll}1 & 13 \quad 17\end{array}$

$1.2978 E-03$

$114 \quad 19$

$9.6787 E-04$

11719

$-8.2616 E-02$ $\begin{array}{lllllllll}1 & 1 & 6 & 1 & 1 & 7 & 1 & 1 & 8\end{array}$

$1 \cdot 1261 E-03$

$$
1112
$$

$5.2053 E-07$

1118

0.

128

$7.8912 E-04$

1214

$8.5492 E-08$

135

$2 \cdot 2.414 E-02$

1311

$8.4130 E-06$ 1317

0.

149

$5.8202 E-03$

1415 .

$4 \cdot 1474 \mathrm{E}-08$

158

$1.8781 E-02$

1514

$3.2424 E-07$

168

$5.0827 E-02$

1614

4.1473E-07

179

$1.1906 E-01$

1715

6.2904E-07

1811

$8.2707 E-04$ 1817

$3.4122 E-07-7.7485 E-07$

1914

$5.6321 E-06$

11012

$1.4918 \mathrm{E}-02$

11018

1.3531E-06

11117

9.3819E-06

11217

$8.4046 E-05$

11318

$2.7067 \mathrm{E}-04$

$\begin{array}{lll}1 & 1 & 17\end{array}$

$8.94 \quad 6 E-02$
5.3409E-04 2.5398E-04

$\begin{array}{llllll}1 & 1 & 13 & 1 & 1 & 14\end{array}$

$1.7351 E-0 ;-2.3767 E-07$

$1119 \quad 1 \quad 24$

$0 . \quad 1.5715 \mathrm{E}-02$

$\begin{array}{lllll}129 & 2 & 10\end{array}$

6.8194E-04 3.9433E-05

1215 I 216

1.4961E-07 0 .

$\begin{array}{llllll}1 & 3 & 6 & 1 & 3 & 7\end{array}$

$1.0595 E-02 \quad 5.0104 E-03$

$\begin{array}{llllll}1 & 3 & 12 & 1 & 3 & 13\end{array}$

$1.4936 E-06 \quad 4.5723 E-07$

$\begin{array}{llllll}1 & 3 & 18 & 1 & 3 & 19\end{array}$

0.

1410

0 .

1411

3.0137E-04 1.9327E-05

$1416 \quad 1 \quad 417$

$-2.7477 E-07 \quad 4.8214 E-07$

$\begin{array}{llll}1 & 5 & 9 & 510\end{array}$

$1.6012 \mathrm{E}-02$

1515

$1.0808 \mathrm{E}-07$

169

$4.3296 E-02$

$16 \quad 15$

$2.7648 E-07$

1710

$5.9826 \mathrm{E}-03$

1716

$8.9863 E-08$

$\begin{array}{lll}1 & 8 & 12\end{array}$

5.3117E-05

$$
1818
$$

1915

$1.6217 E-06$

1. 10 i:

$7.8797 t-04$

11019

2.2945E-06

11118

4.5977E-06

11218

2.5669E-05

11319

2.3433E-05

$\begin{array}{ccc}11518 & 11519 \\ 1.6720 E-02 & -1.8019 E-03\end{array}$

$8.1545 E-04$

1516

$1.0808 \mathrm{E}-07$

1610

$2.1854 \mathrm{E}-03$

1616

$6.9121 E-08$

1711

3.1796E-04

1717

$8.9863 E-08$

1813

6.9951E-06

1819

$1.2298 \mathrm{E}-06$

1916

$9.6424 E-07$

$110 \quad 14$

$5.0478 E-05$

11113

$1.5755 E-02$

11119

5.9646E-06

11219

1. $9260 E-05$

11416

7.3520 E-02

11519

$$
\text { . } 8019 E-03
$$


ATOM

CODE

MASS FRACTION

O

$$
3.600000 E+00
$$$$
5.326100 E-01
$$

B
$5.710000 E-04$
RHO (G/CC)
FORMULA

$F$

$5.570000 E+00$

$2.2400022 E-01$

ATOM

CODE

MASS FRACTION
FUEL DENSITY RHOF $(G / C C)$

\section{TEMPERATURE \\ $T$ (DEG K)}

PROBABILITY/PATH (/CM)

$\begin{array}{cc}\text { COLLIDE- } & M U \\ \text { SGIN) } & N U \\ 7.2544 E-03 & 0 . \\ 1.2295 E-02 & 0 . \\ 1.3461 E-02 & 0 . \\ 1.5851 E-02 & 0 . \\ 2.5911 E-02 & 0 . \\ 2.8483 E-02 & 0 . \\ 2.5339 E-02 & 0 . \\ 2.4991 E-02 & 0 . \\ 2.2537 E-02 & 0 . \\ 2.0974 E-02 & 0 . \\ 2.1441 E-02 & 0 . \\ 2.1659 E-02 & 0 . \\ 2.1996 E-02 & 0 . \\ 2.2322 E-02 & 0 . \\ 2.2719 E-02 & 0 . \\ 2.3253 E-02 & 0 . \\ 2.4239 E-02 & 0 . \\ 2.6565 E-02 & 0 . \\ 2.8506 E-02 & 0 .\end{array}$

MULT I PLY NUSGF $(N)$

0 .

0.

0 .

0.

0.

0 .

0 .

0.

0 .

0 .

0.

0.

0.

\section{.}

.

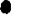

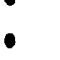

SGSO $(N, N) \quad$ SCATTER

$\begin{array}{llll}5.9193 E-03 & 6.4408 E-04 & 1.3348 E-03 & -4.1392 E-04 \\ 9.8105 E-03 & 1.1949 E-03 & 2.4841 E-03-7.6540 E-04 \\ 1.0804 E-02 & 1.2792 E-03 & 2.6572 E-03-8.2003 E-04 \\ 1.2762 E-02 & 1.4875 E-03 & 3.0885 E-03-9.5397 E-04 \\ 2.0438 E-02 & 2.6290 E-03 & 5.4727 E-03-1.6814 E-03\end{array}$

2.2344E-02 2.9471E-03

2.0009E-02 2.5601E-03

$1.9767 E-02 \quad 2.5097 E-03$

$2.1314 E-02 \quad 1.2191 E-03$

$1.9799 E-02$

$1.1639 E-03$

$6.1386 E-03-1.8837 E-03$

$5.3286 E-03-1.6376 E-03$

$5.2228 E-03-1.6057 E-03$

$1.2194 E-03-3.7406 E-04$

$1.1642 E-03-3.5656 E-04$

$2.0232 E-02$

2.0395E-02

2. $0589 E-02$

$2.0277 \mathrm{E}-02$

1. $9469 E-0$ ?

$1.1797 E-03$

$1.1856 E-03$

$1.192 .7 E-03$

$1.3181 \mathrm{E}-03$

$1.5620 E-03$

$1.1800 E-03-3.6156 E-04$

$1.1859 E-03-3.6343 E-04$

$1.1930 E-03-3.6568 E-04$

$1.5948 E-03-4.8891 E-04$

$2.3915 E-03-7.3310 E-04$

$1.9448 E-02$

1. $9505 E-02$

1. $5610 E-03$

$1.5677 E-03$

2.0296E-02

$1.5899 \mathrm{E}-03$

$2.3477 E-02$

$2.3899 E-03-7.3260 E-04$

$2.4003 E-03-7.3574 E-04$

$2.3653 E-03-7.24 .79 E-04$ 


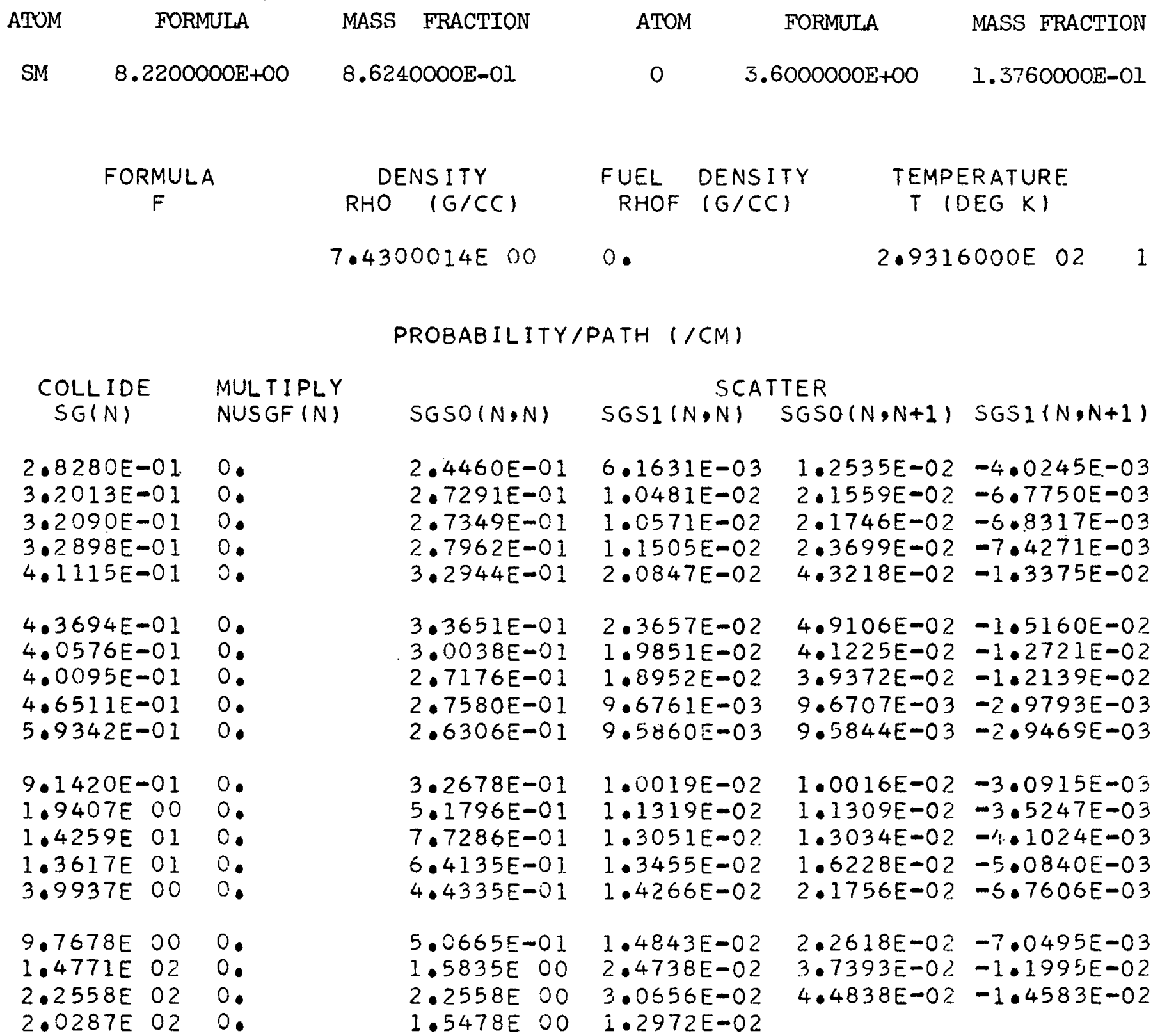


MASS FRACTION

ATOM

CODE

MASS FRACTION

SI

$$
5.500000 E+00 \quad 7.002600 E-01
$$

C

$3.300000 E+00$

$2.997400 E-01$

FORMULA

$F$

$5.530000 E+00$
DENSITY

RHO $(G / C C)$
FUEL DENSITY
RHOF

0.

\section{TEMPERATURE \\ $T$ (DEG K)}

$3.2100009 E 00$

PROBABILITY/PATH (/CM)

$\begin{array}{cc}\text { COLLIDE } & \text { MUL } \\ \text { SGIN) } & \text { NUS } \\ 1.5843 E-01 & 0 . \\ 1.7555 E-01 & 0 . \\ 2.1678 E-01 & 0 . \\ 2.5632 E-01 & 0 . \\ 2.9057 E-01 & 0 . \\ 3.1060 E-01 & 0 . \\ 3.4485 E-01 & 0 . \\ 3.6825 E-01 & 0 . \\ 3.3645 E-01 & 0 . \\ 3.1477 E-01 & 0 . \\ 3.2444 E-01 & 0 . \\ 3.2811 E-01 & 0 . \\ 3.3260 E-01 & 0 . \\ 3.3721 E-01 & 0 . \\ 3.3984 E-01 & 0 . \\ & \\ 3.3999 E-01 & 0 . \\ 3.4007 E-01 & 0 . \\ 3.4191 E-01 & 0 . \\ 3.3357 E-01 & 0 . \\ & \end{array}$

SCATTER

SGSO $(N, N) \quad$ SGSI(N,N) SGSO(N,N+1) SGSI(N,N+I)

$1.2396 E-01 \quad 1.6449 E-02$

$1.3824 E-01 \quad 1.7819 E-02$

$1.7047 E-01 \quad 2.2117 E-02$

2.0296E-01 2.5513E-02

$2.2811 E-01 \quad 2.9823 E-02$

$2.4011 E-01$

$2.6535 E-01$

2. $8212 E-01$

3. $1510 E-0.1$

$2.9364 E-01$

$3.3585 E-02$

$3.7853 E-02$

4. $0985 E-02$

$2.1337 E-02$

$2.1109 E-02$

3.0295E-01

$3.0644 E-01$

$2.1447 E-02$

$2.1575 \mathrm{E}-02$

$2.1727 \mathrm{E}-02$

$2.4177 E-02$

3.0737E-01

$2.9451 E-01$

$2.8795 E-02$

$2.9407 E-01$

$2.9317 E-01$

$2.8 .773 E-02$

$2.8728 E-02$

$2.8142 E-02$

$2.9538 E-0 I$

$3.2787 E-01$
$3.4468 E-02-1.0447 E-02$

$3.7302 E-02-1.1329 E-02$

4.6309E-02 - $1.4058 E-02$

$5.3365 E-02-1.6235 E-02$

$6.2459 E-02-1.8950 E-02$

$7.0494 E-02-2.1291 E-02$

$7.9502 E-02-2.3980 E-02$

$8.6129 E-02-2.5948 E-02$

$2.1345 E-02-6.3910 E-03$

$2.1117 E-02-6.2975 E-03$

2. $1456 E-02-6.4049 E-03$

$2.1583 E-02-6.4452 E-03$

$2.1736 E-02-6.4936 E-03$

$2.9327 E-02-8.7624 E-03$

4.4357E-02 - $1.3251 E-02$

$4.4323 E-02-1.3240 E-02$

4.4255E-02 - $1.3219 E-02$

$4.2104 E-02-1.2573 E-02$
$1.5080 E-02$ 


\begin{tabular}{|c|c|c|c|c|c|}
\hline ATOM & CODE & MASS FRACTION & ATOM & CODE & MASS FRACTION \\
\hline 0 & $3.600000 E+00$ & $5.328000 E-01$ & SI & $5.500000 E+00$ & $4.672000 E-01$ \\
\hline & $\begin{array}{c}\text { FORMULA } \\
\mathrm{F}\end{array}$ & $\begin{array}{cc} & \text { DENSITY } \\
\text { RHO } \quad(G / C C)\end{array}$ & $\begin{array}{l}\text { FUEL } \\
\text { RHOF }\end{array}$ & $\begin{array}{l}\text { DENSITY } \\
(G / C C)\end{array}$ & $\begin{array}{l}\text { 1PERATURE } \\
\text { (DEG K) }\end{array}$ \\
\hline & $10000 E+00$ & $2.6499994 \mathrm{E} \quad 00$ & 0. & 2.93 & $316000 E \quad 02$ \\
\hline
\end{tabular}

PROBABILITY/PATH (/CM)

\begin{tabular}{|c|c|c|c|c|c|}
\hline COLLIDE & MULTIPLY & & $S C A$ & TER & \\
\hline$S G(N)$ & NUSGF $(N)$ & $\operatorname{SGSO}(N, N)$ & $S G S I(N, N)$ & $\operatorname{SGSO}(N, N+1)$ & SGSI $1(N, N+1)$ \\
\hline $\begin{array}{l}8.5534 E-02 \\
1.4519 E-01 \\
1.5900 E-01 \\
1.8729 E-01 \\
3.0634 E-01\end{array}$ & $\begin{array}{l}0 . \\
0 . \\
0 . \\
0 .\end{array}$ & $\begin{array}{l}6.9853 E-02 \\
1.1591 E-01 \\
1.2767 E-01 \\
1.5085 E-01 \\
2.4169 E-01\end{array}$ & $\begin{array}{l}7.5687 E-03 \\
1.4088 E-02 \\
1.5086 E-02 \\
1.7551 E-02 \\
3.1060 E-02\end{array}$ & $\begin{array}{l}1.5682 E-02 \\
2.9284 E-02 \\
3.1333 E-02 \\
3.6439 E-02 \\
6.4655 E-02\end{array}$ & $\begin{array}{l}-4.8653 E-03 \\
-9.0250 E-03 \\
-9.6718 E-03 \\
-1.1257 E-02 \\
-1.9867 E-0 ?\end{array}$ \\
\hline $\begin{array}{l}3.3678 E-01 \\
2.9957 E-01 \\
2.9545 E-01 \\
2.6637 E-01 \\
2.4779 E-01\end{array}$ & $\begin{array}{l}0 . \\
0 . \\
0 . \\
0 .\end{array}$ & $\begin{array}{l}2.6424 E-01 \\
2.3662 E-01 \\
2.3375 E-01 \\
2.5197 E-01 \\
2.3403 E-01\end{array}$ & $\begin{array}{l}3.4825 E-02 \\
3.0245 E-02 \\
2.9649 E-02 \\
1.4399 E-02 \\
1.3746 E-02\end{array}$ & $\begin{array}{l}7.2535 E-02 \\
6.2949 E-02 \\
6.1698 E-02 \\
1.4402 E-02 \\
1.3749 E-02\end{array}$ & $\begin{array}{l}-2.2260 E-02 \\
-1.9348 E-02 \\
-1.8970 E-02 \\
-4.4186 E-03 \\
-4.2114 E-03\end{array}$ \\
\hline $\begin{array}{l}2.5311 E-01 \\
2.5513 E-01 \\
2.5760 E-01 \\
2.5881 E-01 \\
2.5893 E-01\end{array}$ & $\begin{array}{l}0 . \\
0 . \\
0 . \\
0 .\end{array}$ & $\begin{array}{l}2.3916 E-01 \\
2.4108 E-01 \\
2.4338 E-01 \\
2.3970 E-01 \\
2.3016 E-01\end{array}$ & $\begin{array}{l}1.3932 E-02 \\
1.4003 E-02 \\
1.4087 E-02 \\
1.5568 E-02 \\
1.8449 E-02\end{array}$ & $\begin{array}{l}1.3936 E-02 \\
1.4006 E-02 \\
1.4090 E-02 \\
1.8836 E-02 \\
2.8245 E-02\end{array}$ & $\begin{array}{l}-4.2705 E-03 \\
-4.2928 E-03 \\
-4.3194 E-03 \\
-5.7750 E-03 \\
-8.6594 E-03\end{array}$ \\
\hline $\begin{array}{l}2.5900 E-01 \\
2.6036 E-01 \\
2.7026 E-01 \\
2.8061 E-01\end{array}$ & $\begin{array}{l}0 . \\
0 . \\
0 .\end{array}$ & $\begin{array}{l}2.2991 E-01 \\
2.3059 E-01 \\
2.3994 E-01 \\
2.7753 E-01\end{array}$ & $\begin{array}{l}1.8437 E-02 \\
1.8517 E-02 \\
1.8780 E-02 \\
1.0638 E-02\end{array}$ & $\begin{array}{l}2.8226 E-02 \\
2.8349 E-02 \\
2.7938 E-02\end{array}$ & $\begin{array}{l}-8.6535 E-03 \\
-8.6906 E-03 \\
-8.5618 E-03\end{array}$ \\
\hline
\end{tabular}


*MIID STEEL

*MILD STEEL

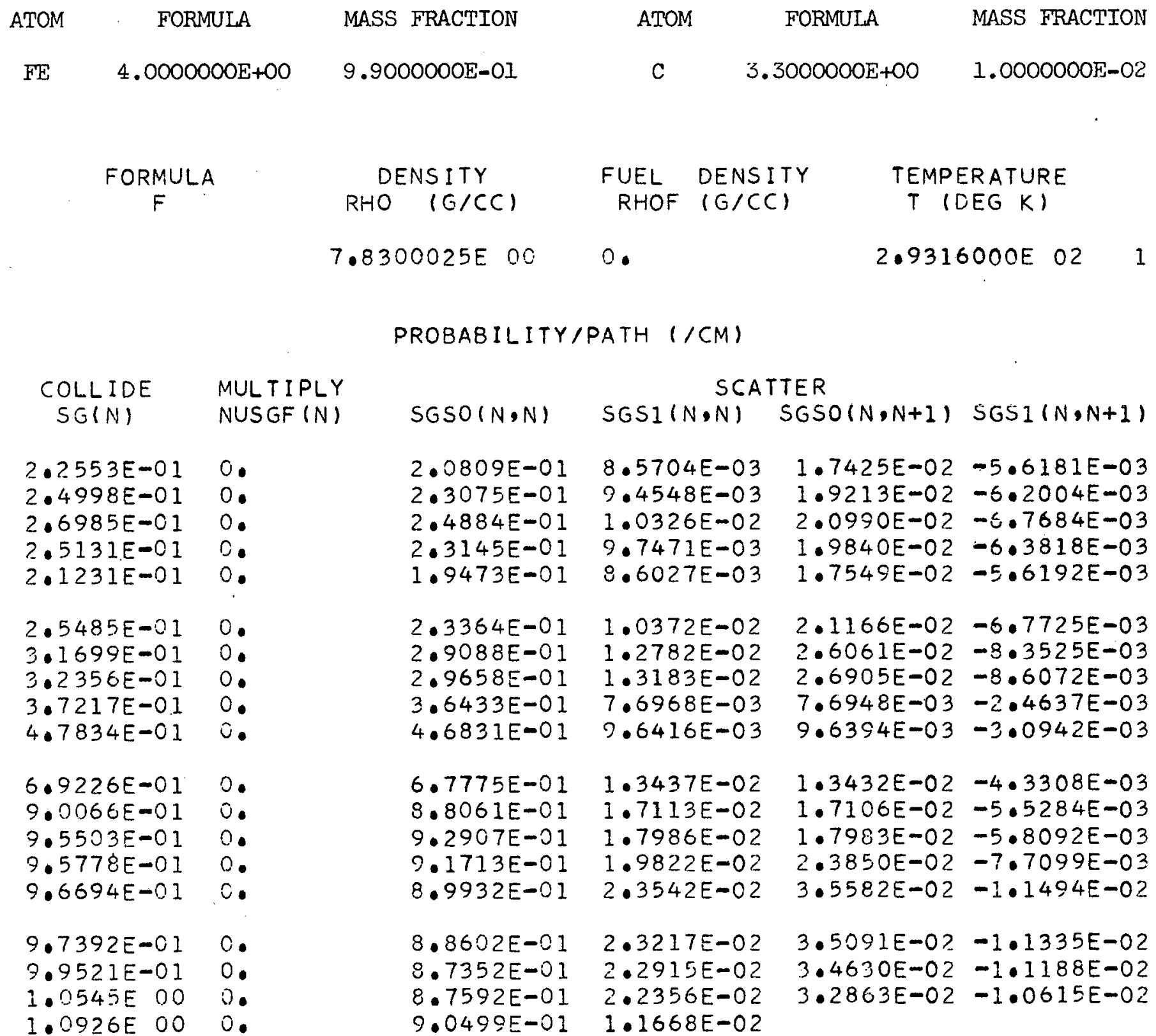




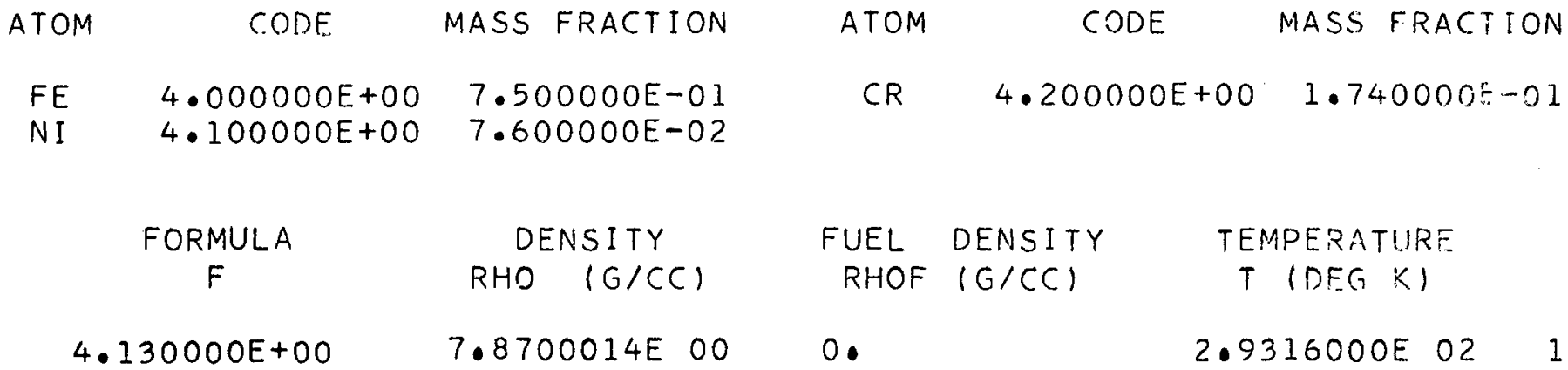

PROBABILITY/PATH (/CM)

$\begin{array}{cc}\text { COLLIDE } & \text { MULT } \\ \text { SGIN) } & \text { NUS } \\ 2.0687 E-01 & 0 . \\ 2.2657 E-01 & 0 . \\ 2.4119 E-01 & 0 . \\ 2.2625 E-01 & 0 . \\ 1.9677 E-01 & 0 . \\ 2.3139 E-01 & 0 . \\ 2.8497 E-01 & 0 . \\ 2.9763 E-01 & 0 . \\ 3.6901 E-01 & 0 . \\ 5.6407 E-01 & 0 . \\ 7.5455 E-01 & 0 . \\ 8.5790 E-01 & 0 . \\ 8.9992 E-01 & 0 . \\ 9.0435 E-01 & 0 . \\ 9.1375 E-01 & 0 . \\ 9.2562 E-01 & 0 . \\ 9.4745 E-01 & 0 . \\ 1.0069 E-00 & 0 . \\ 1.0548 E-00 & 0 .\end{array}$

SGSO $(N, N) \quad$ SGSI(N,N) SGSO $(N, N+1)$ SGSI(N,N+I)

$1.9207 E-01 \quad 7.3054 E-03$

2.1037E-01 7.9976E-03

2.2395E-01 8.5137E-03

$2.1006 E-01 \quad 7.9844 E-03$

$1.8267 \mathrm{E}-01 \quad 6.9506 \mathrm{E}-03$

$1.4783 E-02-4.8117 E-02$

$1.6181 E-02-5.2682 E-03$

$1.7219 E-02-5.6091 E-03$

$1.6156 E-02-5.2591 E-03$

$1.4060 E-02-4.5789 E-03$

$2.1482 E-01 \quad 8.1670 E-03 \quad 1.6523 E-02-5.3798 E-03$

$2.6459 E-01 \quad 1.0050 E-02 \quad 2.0325 E-02-6.6212 E-03$

$2.7634 E-01 \quad 1.0487 E-02 \quad 2.1218 E-02-6.9081 E-03$

$3.6227 E-01 \quad 6.5795 E-03 \quad 6.5774 E-03-2.1417 E-03$

$5.5352 E-01 \quad 1.0119 E-02 \quad 1.0117 E-02-3.2929 E-03$

7.3986E-01 1.3487E-02 1.3483E-02 -4.3897E-03

$8.3943 E-01 \quad 1.5208 E-02 \quad 1.5202 E-02-4.9521 E-03$

$8.7521 \mathrm{E}-01 \quad 1.5849 \mathrm{E}-02 \quad 1.5845 \mathrm{E}-02-5.1584 \mathrm{E}-03$

$8.6466 E-01 \quad 1.7468 E-02 \quad 2.1000 E-02-6.8417 E-03$

$8.4689 E-01 \quad 2.0707 E-02 \quad 3.1235 E-02-1.0170 E-02$

$8.3604 E-01 \quad 2.0447 E-02 \quad 3.0840 E-02-1.0044 E-02$

$8.2033 E-01 \quad 2.0075 E-02 \quad 3.0277 E-02-9.861 I E-03$

$8.1638 E-01 \quad 1.9434 E-02 \quad 2.8515 E-02-9.2852 E-03$ 


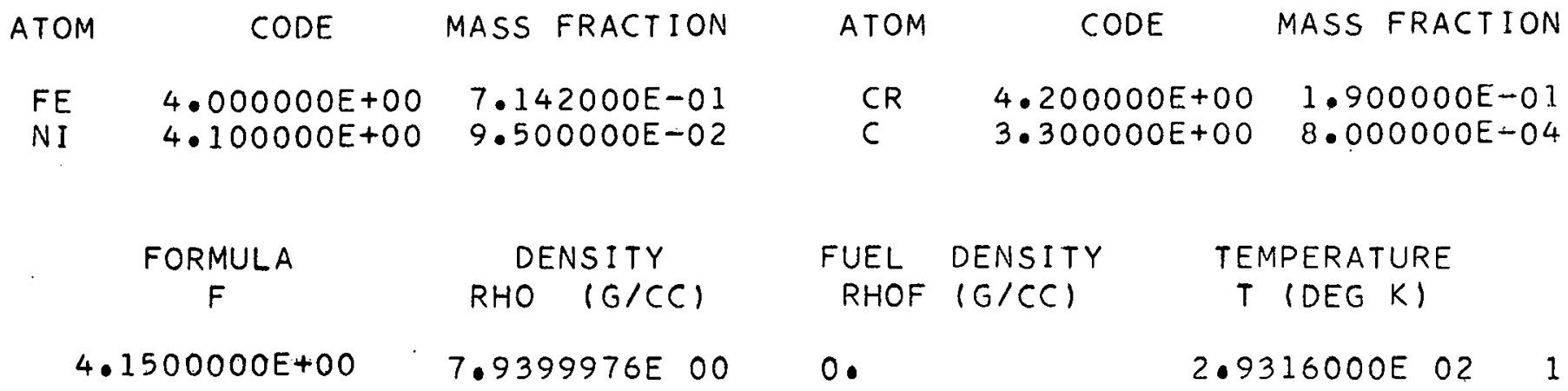

PROBABILITY/PATH (/CM)

\begin{tabular}{|c|c|c|c|c|c|}
\hline $\begin{array}{l}\text { COLLIDE } \\
\text { SG(N) }\end{array}$ & $\begin{array}{l}\text { MULTIPLY } \\
\text { NUSGF (N) }\end{array}$ & $\operatorname{SGSO}(N, N)$ & $\operatorname{SGSI}(N, N)$ & $\begin{array}{l}\text { TER } \\
\text { SGSO }(N, N+1)\end{array}$ & $\operatorname{SGS} 1(N, N+1)$ \\
\hline $\begin{array}{l}2.0750 E-01 \\
2.2681 E-01 \\
2.4116 E-01 \\
2.2677 E-01 \\
1.9900 E-01\end{array}$ & $\begin{array}{l}0 . \\
0 . \\
0 . \\
0 . \\
0 .\end{array}$ & $\begin{array}{l}1.9254 E-01 \\
2.1048 E-01 \\
2.2378 E-01 \\
2.1039 E-01 \\
1.8454 E-01\end{array}$ & $\begin{array}{l}7.3788 E-03 \\
8.0585 E-03 \\
8.5785 E-03 \\
8.0753 E-03 \\
7.1196 E-03\end{array}$ & $\begin{array}{l}1.4939 E-02 \\
1.6311 E-02 \\
1.7360 E-02 \\
1.6350 E-02 \\
1.4414 E-02\end{array}$ & $\begin{array}{l}-4.8579 E-03 \\
-5.3060 E-03 \\
-5.6488 E-03 \\
-5.3159 E-03 \\
-4.6863 E-03\end{array}$ \\
\hline $\begin{array}{l}2.3341 E-01 \\
2.8711 E-01 \\
3.0168 E-01 \\
3.7870 E-01 \\
5.8831 E-01\end{array}$ & $\begin{array}{l}0 . \\
0 \\
0 \\
0 \\
0\end{array}$ & $\begin{array}{l}2.1645 E-01 \\
2.6630 E-01 \\
2.7979 E-01 \\
3.7170 E-01 \\
5.7722 E-01\end{array}$ & $\begin{array}{l}8.3506 E-03 \\
1.0253 E-02 \\
1.0770 E-02 \\
6.8343 E-03 \\
1.0641 E-02\end{array}$ & $\begin{array}{l}1.6910 E-02 \\
2.0755 E-02 \\
2.1810 E-02 \\
6.8323 E-03 \\
1.0639 E-02\end{array}$ & $\begin{array}{l}-5.4959 E-03 \\
-6.7496 E-03 \\
-7.0884 E-03 \\
-2.2212 E-03 \\
-3.4590 E-03\end{array}$ \\
\hline $\begin{array}{l}7.7868 E-01 \\
8.7091 E-01 \\
9.1167 E-01 \\
9.1637 E-01 \\
9.2567 E-01\end{array}$ & $\begin{array}{l}0 . \\
0 . \\
0 . \\
0 . \\
0 .\end{array}$ & $\begin{array}{l}7.6345 E-01 \\
8.5205 E-01 \\
8.8645 E-01 \\
8.7585 E-01 \\
8.5739 E-01\end{array}$ & $\begin{array}{l}1.4003 E-02 \\
1.5517 E-02 \\
1.6132 E-02 \\
1.7783 E-02 \\
2.1 .071 E-02\end{array}$ & $\begin{array}{l}1.3999 E-02 \\
1.5510 E-02 \\
1.6128 E-02 \\
2.1380 E-02 \\
3.1790 E-02\end{array}$ & $\begin{array}{l}-4.5539 E-03 \\
-5.0489 E-03 \\
-5.2472 E-03 \\
-6.9606 E-03 \\
-1.0343 E-02\end{array}$ \\
\hline $\begin{array}{l}9.3819 E-01 \\
9.5942 E-01 \\
1.0188 E \quad 00 \\
1.0687 E \quad 00\end{array}$ & $\begin{array}{l}0 . \\
0 . \\
0 . \\
0 .\end{array}$ & $\begin{array}{l}8.4662 E-01 \\
8.2944 E-0 I \\
8.2386 E-0 I \\
8.5493 E-0 I\end{array}$ & $\begin{array}{l}2.0814 E-02 \\
2.0408 E-02 \\
1.9723 E-02 \\
1.0327 E-02\end{array}$ & $\begin{array}{l}3.1398 E-02 \\
3.0785 E-02 \\
2.8943 E-02\end{array}$ & $\begin{array}{l}-1.0219 E-02 \\
-1.0019 E-02 \\
-9.4174 E-03\end{array}$ \\
\hline
\end{tabular}


ATOM

CODE

MASS FRACTION

$\begin{array}{cc}\text { FE } & 4.000000 \\ \text { NI } & 4.100000 \\ & \text { FORMULA } \\ & \text { F }\end{array}$

$4 \cdot 1700000 E+00$
$5.590000 E-0.1$

1. $910000 E-01$
DENSITY

RHO (G/CC)
$7.9799978 E \quad 00$
FUEL DENSITY
RHOF $(G / C C)$

0 .
ATOM

CODE

MASS FRACTION

CR $\quad 4.200000 E+00 \quad 2.500000 E-0.1$

\author{
$T$ (OFG K)
}

TEMPERATURF

$2.9316000 E \quad 02 \quad 1$

PROBABILITY/PATH (/CM)

$\begin{array}{cc}\text { COLLIDE } & \text { MUL } \\ \text { SG(N) } & \text { NUS } \\ 2.0273 E-01 & 0 . \\ 2.1982 E-01 & 0 . \\ 2.3198 E-01 & 0 . \\ 2.2014 E-01 & 0 . \\ 2.0007 E-01 & 0 . \\ 2.3175 E-01 & 0 . \\ 2.8437 E-01 & 0 . \\ 3.0717 E-01 & 0 . \\ 4.0858 E-01 & 0 . \\ 6.7717 E-01 & 0 . \\ 8.6435 E-01 & 0 . \\ 9.0864 E-01 & 0 . \\ 9.4290 E-01 & 0 . \\ 9.4860 E-01 & 0 . \\ 9.5672 E-01 & 0 . \\ 9.7163 E-01 & 0 . \\ 9.8836 E-01 & 0 . \\ 1.0446 E-00 & 0 . \\ 1.1033 E-00 & 0 .\end{array}$

MULT IPLY

NUSGF $(N)$

\begin{abstract}
$\operatorname{SGSO}(N, N)$
$1.8825 E-01$

$2.0413 E-01$

$2.1542 E-01$

$2.0441 E-01$

$1.8577 \mathrm{E}-01$

2.1519E-01

$2.6408 E-01$

2.8527E-01

4.0115E-01

$6.6459 E-01$

$8.4764 E-01$

$8.8901 E-01$

$9.1658 E-01$

$9.0602 E-01$

$8.8481 E-01$

$8.7448 E-01$

$8.4987 E-01$

$8.3607 E-01$

$8.7203 E-01$
\end{abstract}

SCATTER

SGSI N,N) SGSO $(N, N+1)$ SGSI $(N, N+1)$

$7.1478 E-03$

$7 \cdot 7447 E-03$

$8.1718 E-03$

$7.7538 E-03$

$7.0502 E-03$

$1.4464 E-02-4.7080 E-03$

$1.5671 E-02-5.1014 E-03$

$1.6531 E-02-5.3335 E-03$

$1.5690 E-02-5.1073 E-03$

$1.4261 E-02-4.6447 E-03$

8.1593E-03

$9.9949 E-03$

$1.0778 E-02$

$7.2461 E-03$

$1.2098 \mathrm{E}-02$.

$1.6507 E-02-5.3749 E-03$

$2.0216 E-02-6.5850 E-03$

2.1807E-02 -7.1003E-03

$7.2443 F-03-2.3585 E-03$

1.2096E-02 $-3.9365 E-03$

$1.5384 E-02$

$1.6010 E-02$

$1.6500 \mathrm{E}-02$

$1.8194 E-02$

$2 \cdot 1506 E-0$ ?

$1.5380 E-02-5.0063 E-03$

$1.6003 E-02-5.2131 \mathrm{~F}-03$

$1.6495 E-02-5.371 .5 E-03$

$2.1873 E-02-7.1269 E-03$

$3.2440 E-02-1.0563 E-02$

$2.1265 E-0 \% \quad 2.2069 E-0 \%-1.0443 E-02$

2.0686E-02 $.1198 E-0 ?-1.016 ? E-0 ?$

$1.9805 E-02 \quad 2.9059 E-02-0.4627 E-03$

(1) 


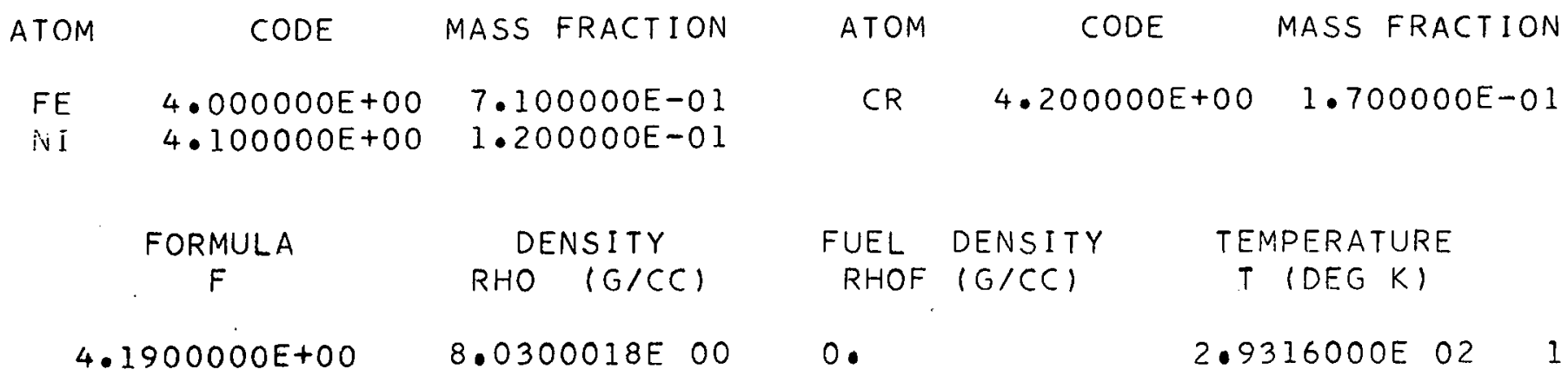

PROBABILITY/PATH (/CM)

$$
\begin{array}{cl}
\text { COLLIDE } & \text { MULTIPLY } \\
\text { SG }(N) & \text { NUSGF }(N)
\end{array}
$$

\begin{tabular}{|c|c|c|c|}
\hline \multicolumn{4}{|c|}{ SCATTER } \\
\hline $5 G S O(N, N)$ & $\operatorname{SGSI}(N, N)$ & SGSO $(N, N+1)$ & $\operatorname{SGSI}(N, N+1)$ \\
\hline $\begin{array}{l}1.9650 E-01 \\
2.1491 E-01 \\
2.2840 E-01 \\
2.1480 E-01 \\
1.8892 E-01\end{array}$ & $\begin{array}{l}7.4564 E-03 \\
8.1515 E-03 \\
8.6634 E-03 \\
8.1457 E-03 \\
7.1685 E-03\end{array}$ & $\begin{array}{l}1.5088 E-02 \\
1.6492 E-02 \\
1.7522 E-02 \\
1.6482 E-02 \\
1.4500 E-02\end{array}$ & $\begin{array}{l}-4.9113 E-03 \\
-5.3696 E-03 \\
-5.7077 E-03 \\
-5.3655 E-03 \\
-4.7227 E-03\end{array}$ \\
\hline $\begin{array}{l}2.2148 E-01 \\
2.7329 E-01 \\
2.8859 E-01 \\
3.8629 E-01 \\
5.9693 E-01\end{array}$ & $\begin{array}{l}8.3984 E-03 \\
1.0352 E-02 \\
1.0918 E-02 \\
6.9890 E-03 \\
1.0863 E-02\end{array}$ & $\begin{array}{l}1.6991 E-02 \\
2.0937 E-02 \\
2.2089 E-02 \\
6.9869 E-03 \\
1.0860 E-02\end{array}$ & $\begin{array}{l}-5.5325 E-03 \\
-6.8207 E-03 \\
-7.1922 E-03 \\
-2.2750 E-03 \\
-3.5350 E-03\end{array}$ \\
\hline $\begin{array}{l}7.9092 E-01 \\
8.8799 E-01 \\
9.2325 E-01 \\
9.1222 E-01 \\
8.9244 E-01\end{array}$ & $\begin{array}{l}1.4359 E-02 \\
1.6034 E-02 \\
1.6665 E-02 \\
1.8368 E-02 \\
2.1749 E-02\end{array}$ & $\begin{array}{l}1.4354 E-02 \\
1.6027 E-02 \\
1.6660 E-02 \\
2.2082 E-02 \\
3.2807 E-02\end{array}$ & $\begin{array}{l}-4.6736 E-03 \\
-5.2211 E-03 \\
-5.4245 E-03 \\
-7.1949 E-03 \\
-1.0682 E-02\end{array}$ \\
\hline $\begin{array}{l}8.8102 E-01 \\
8.6093 E-01 \\
8.5305 E-01 \\
8.8598 E-01\end{array}$ & $\begin{array}{l}2.1478 E-02 \\
2.1002 E-02 \\
2.0246 E-02 \\
1.0609 E-02\end{array}$ & $\begin{array}{l}3.2392 E-02 \\
3.1675 E-02 \\
2.9706 E-02\end{array}$ & $\begin{array}{l}-1.0551 E-02 \\
-1.0317 E-02 \\
-9.6736 E-03\end{array}$ \\
\hline
\end{tabular}

$\begin{array}{ll}2.1160 E-01 & 0 . \\ 2.3142 E-01 & 0 . \\ 2.4594 E-01 & 0 . \\ 2.3131 E-01 & 0 . \\ 2.0346 E-01 & 0 . \\ 2.3852 E-01 & 0 . \\ 2.9429 E-01 & 0 . \\ 3.1076 E-01 & 0 . \\ 3.9345 E-01 & 0 . \\ 6.0825 E-01 & 0 . \\ 8.0653 E-01 & 0 . \\ 9.0744 E-01 & 0 . \\ 9.4922 E-01 & 0 . \\ 9.5391 E-01 & 0 . \\ 9.6263 E-01 & 0 . \\ 9.7505 E-01 & 0 . \\ 9.9422 E-01 & 0 . \\ 1.0528 E 00 & 0 . \\ 1.1050 E-00 & 0 .\end{array}$


\#410 STAINLESS STEEL

$* 410 \$ 5$

\begin{tabular}{|c|c|c|c|c|c|}
\hline TOM & CODE & MASS FRACTION & ATOM & CODE & MASS FRACTION \\
\hline $\begin{array}{l}\text { FE } \\
\text { NI }\end{array}$ & $\begin{array}{l}4 \cdot 000000 E+00 \\
4 \cdot 100000 E+00 \\
3 \cdot 300000 E+00\end{array}$ & $\begin{array}{l}6.840000 E-01 \\
1.200000 E-01 \\
1.000000 E-03\end{array}$ & $\begin{array}{l}C R \\
M O\end{array}$ & $\begin{array}{l}4.200000 E+00 \\
5.300000 E+00\end{array}$ & $\begin{array}{l}1.700000 E-01 \\
2.500000 E-02\end{array}$ \\
\hline & $\begin{array}{c}\text { FORMULA } \\
\text { F }\end{array}$ & $\begin{array}{c}\text { DENSITY } \\
\text { RHO }(G / C C)\end{array}$ & $\begin{array}{l}\text { FUEL } \\
\text { RHOF }\end{array}$ & $\begin{array}{l}\text { DENSITY } \\
(G / C C)\end{array}$ & $\begin{array}{l}\text { MPERATURE } \\
\text { (DEG K) }\end{array}$ \\
\hline & $00000 E+00$ & $7.7000014 \mathrm{E} 00$ & 0. & 2.9 & $316000 E \quad 02$ \\
\hline
\end{tabular}

PROBABILITY/PATH (/CM)

$\begin{array}{cc}\text { COLLIDE } & \text { MU } \\ \text { SGIN) } & \text { NU } \\ 1.9992 E-01 & 0 . \\ 2.1971 E-01 & 0 . \\ 2.3467 E-01 & 0 . \\ 2.2262 E-01 & 0 . \\ 1.9852 E-01 & 0 . \\ 2.3265 E-01 & 0 . \\ 2.8616 E-01 & 0 . \\ 3.0251 E-01 & 0 . \\ 3.7990 E-01 & 0 . \\ 5.8228 E-01 & 0 . \\ 7.6966 E-01 & 0 . \\ 8.7023 E-01 & 0 . \\ 9.0947 E-01 & 0 . \\ 9.0420 E-01 & 0 . \\ 9.0827 E-01 & 0 . \\ 9.2021 E-01 & 0 . \\ 9.3850 E-01 & 0 . \\ 9.9374 E-01 & 0 . \\ 1.0427 E-00 & 0 .\end{array}$

SGSO (N,N) SGSI(N,N) SGSO $(N, N+1) \quad S G S I(N, N+1)$

$1.8558 E-01 \quad 7.0773 E-03 \quad 1.4329 E-02-4.6591 E-03$

$2.0399 E-01 \quad 7.7538 E-03 \quad 1.5694 E-02-5.1053 E-03$

$2.1789 \mathrm{E}-01 \quad 8.2777 \mathrm{E}-03 \quad 1.6751 \mathrm{E}-02-5.4507 \mathrm{E}-03$

$2.0672 E-01 \quad 7.8388 E-03 \quad 1.5870 E-02-5.1603 E-03$

$1.8432 E-01 \quad 6.9958 E-03 \quad 1.4162 E-02-4.6049 E-03$

2.1599E-01 8.2015E-03 1.6606E-02 -5.3979E-03

$2.6569 E-01 \quad 1.0077 E-02 \quad 2.0398 E-02-6.6333 E-03$

$2.8086 E-01 \quad 1.0649 E-02 \quad 2.1564 E-02-7.0090 E-03$

$3.7293 E-01 \quad 6.7802 E-03 \quad 6.7781 E-03-2.2034 E-03$

$5.7133 E-01 \quad 1.0444 E-02 \quad 1.0441 E-02-3.3947 E-03$

$7.5412 E-01 \quad 1.3739 E-02 \quad 1.3735 E-02-4.4679 E-03$

$8.4694 E-01 \quad 1.5295 E-02 \quad 1.5288 E-02-4.9769 E-03$

$8.7877 E-01 \quad 1.5878 E-0 ? \quad 1.5873 E-02-5.1646 E-03$

$8.6283 E-01 \quad 1.7443 E-02 \quad 2.0972 E-02-6.8276 E-03$

$8.4173 E-01 \quad 2.0584 E-02 \quad 3.1056 E-02-1.0104 E-02$

$8.3104 E-01 \quad 2.0332 E-02 \quad 3.0670 E-02-9.9818 E-03$

8.1206E-01 1.9884E-02 2.9995E-02 -9.7619E-03

$8.0424 E-01 \quad 1.9167 E-02 \quad 2.812 .9 E-02-9.1519 E-03$

$8.3480 E-01 \quad 1.0044 E-02$ 


\begin{tabular}{|c|c|c|c|c|c|}
\hline ATOM & CODE & MASS FRACTION & ATOM & CODE & MASS FRACTION \\
\hline $\begin{array}{l}F F \\
C\end{array}$ & $\begin{array}{l}4.000000 F+00 \\
3.300000 F+00\end{array}$ & $\begin{array}{l}8.388000 E-01 \\
1.200000 E-03\end{array}$ & $C R$ & $4.200000 E+00$ & 1. $600000 E-01$ \\
\hline & $\begin{array}{c}\text { FORMULA } \\
\mathrm{F}\end{array}$ & $\begin{array}{c}\text { DENSITY } \\
\text { RHO }(G / C C)\end{array}$ & $\begin{array}{l}\text { FUEL } \\
\text { RHOF }\end{array}$ & $\begin{array}{l}\text { DENSITY } \\
(G / C C)\end{array}$ & TEMPERATURE \\
\hline & $300000 E+00$ & $7.7499983 E \quad 00$ & 0. & 2.93 & $316000 E \quad 02$ \\
\hline
\end{tabular}

PROBABILITY/PATH (/CM)

\begin{tabular}{|c|c|c|c|c|c|}
\hline \multirow{2}{*}{$\begin{array}{l}\text { COLLIDE } \\
\text { SG }(N)\end{array}$} & \multirow{2}{*}{$\begin{array}{l}\text { MULT IPLY } \\
\operatorname{NUSGF}(N)\end{array}$} & \multicolumn{4}{|c|}{ SCATTER } \\
\hline & & $\operatorname{SGSO}(N, N)$ & $\operatorname{SGSI}(N, N)$ & $\operatorname{SGSO}(N, N+1)$ & $\operatorname{SGSI}(N, N+1)$ \\
\hline $\begin{array}{l}2.0522 E-01 \\
2.2558 E-01 \\
2.4119 E-01 \\
2.2514 E-01 \\
1.9174 E-01\end{array}$ & $\begin{array}{l}0 \\
0 \\
0 \\
0 \\
0\end{array}$ & $\begin{array}{l}1.9033 E-01 \\
2.0924 E-01 \\
2.2369 E-01 \\
2.0876 E-01 \\
1.7767 E-01\end{array}$ & $\begin{array}{l}7.3427 E-03 \\
8.0631 E-03 \\
8.6350 E-03 \\
8.0754 E-03 \\
6.9309 E-03\end{array}$ & $\begin{array}{l}1.4869 E-02 \\
1.6323 E-02 \\
1.7476 E-02 \\
1.6355 E-02 \\
1.4039 E-02\end{array}$ & $\begin{array}{l}-4.8329 E-03 \\
-5.3080 E-03 \\
-5.6849 E-03 \\
-5.3143 E-03 \\
-4.5599 E-03\end{array}$ \\
\hline $\begin{array}{l}2.2713 E-01 \\
2.7930 E-01 \\
2.8586 E-01 \\
3.3906 E-01 \\
4.9782 E-01\end{array}$ & $\begin{array}{l}0 . \\
0 . \\
0 . \\
0 . \\
0 .\end{array}$ & $\begin{array}{l}2.1045 E-01 \\
2.5884 E-0 I \\
2.6486 E-0 I \\
3.3270 E-0 I \\
4.8829 E-0 I\end{array}$ & $\begin{array}{l}8.2106 E-03 \\
1.0079 E-02 \\
1.0331 E-02 \\
6.2101 E-03 \\
9.1296 E-03\end{array}$ & $\begin{array}{l}1.6634 E-02 \\
2.0409 E-02 \\
2.0930 E-02 \\
6.2079 E-03 \\
9.1270 E-03\end{array}$ & $\begin{array}{l}-5.4012 E-03 \\
-6.6320 E-03 \\
-6.7955 E-03 \\
-2.0164 E-03 \\
-2.9653 E-03\end{array}$ \\
\hline $\begin{array}{l}6.8238 E-01 \\
8.0293 E-01 \\
8.4736 E-01 \\
8.5126 E-01 \\
8.6180 E-01\end{array}$ & $\begin{array}{l}0 . \\
0 . \\
0 \\
0 \\
0\end{array}$ & $\begin{array}{l}6.6886 E-01 \\
7.8544 E-0 I \\
8.2397 E-01 \\
8.1381 E-01 \\
7.9873 E-01\end{array}$ & $\begin{array}{l}1.2408 E-02 \\
1.4448 E-02 \\
1.5139 E-02 \\
1.6683 E-02 \\
1.9819 E-02\end{array}$ & $\begin{array}{l}1.2404 E-02 \\
1.4442 E-02 \\
1.5136 E-02 \\
2.0059 E-02 \\
2.9906 E-02\end{array}$ & $\begin{array}{l}-4.0334 E-03 \\
-4.6989 E-03 \\
-4.9212 E-03 \\
-6.5272 E-03 \\
-9.7247 E-03\end{array}$ \\
\hline $\begin{array}{l}8.7246 E-01 \\
8.9850 E-01 \\
9.6019 E-01 \\
1.0013 E \quad 00\end{array}$ & $\begin{array}{l}0 \\
0 \\
0 \\
0\end{array}$ & $\begin{array}{l}7.8826 E-01 \\
7.7916 E-01 \\
7.8165 E-01 \\
8.0704 E-01\end{array}$ & $\begin{array}{l}1.9566 E-02 \\
1.9348 E-02 \\
1.8876 E-02 \\
9.8356 E-03\end{array}$ & $\begin{array}{l}2.9522 E-02 \\
2.9190 E-02 \\
2.7704 E-02\end{array}$ & $\begin{array}{l}-9.6007 E-03 \\
-9.4947 E-03 \\
-9.0099 E-03\end{array}$ \\
\hline
\end{tabular}


*446 STAINIESS STEEL

*446 SS

$\begin{array}{cccccc}\text { IIOM } & \text { CODE } & \text { MASS FRACTION } & \text { ATOM } & \text { CODE } & \text { MASS FRACTION } \\ \text { FE } & 4.000000 E+00 & 6.975000 E-O 1 & \text { NB } & 5.360000 E+00 & 5.000000 E-03 \\ \text { CR } & 4.200000 E+00 & 2.700000 E-O 1 & \text { NI } & 4.100000 E+00 & 5.000000 \mathrm{O}-03 \\ \text { SI } & 4.500000 E+00 & 1.000000 E-02 & \text { N } & 6.300000 E+00 & 2.500000 E-03 \\ \text { MN } & 4.400000 E+00 & 8.000000 E-03 & \text { C } & 3.300000 E+00 & 2.000000 E-03\end{array}$

FORMULA
F

$$
\begin{aligned}
& \text { DENSITY } \\
& \text { RHO }(G / C C)
\end{aligned}
$$
FUEL DENSITY
RHOF (G/CC)

\section{TEMPERATURE \\ $T$ (DEG K)}

$7.6000012 E 00$

0.

$2.9316000 E_{02}$

1

\begin{abstract}
COLL IDE
$S G(N)$

$\begin{array}{ll}1.932 ? E-0 ! & 0 . \\ 2.108 ? F-01 & 0 . \\ 2.2464 E-01 & 0 . \\ 2.1303 E-0 ! & 0 . \\ 1.8712 E-01 & 0 .\end{array}$

$2.1768 E-01 \quad 0$.

$2.6353 E-010$.

2.?2?2E-01 0 .

$3.2985 E-010$.

$5.8836 F-010$.

$7.6280 F-09 \quad 0$.

$7.4947 E-01 \quad 0$.

$7.66225-010$.

$7.7098 E-01$ ग.

$7.8285 E-01 \quad \mathrm{C}$.

7.9682E-01 0.

$8.2669 E-010$.

8.0047E-01 0.

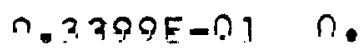

MUL TIPLY

NUSGF (N)

PROBABILITY/PATH (/CM)

\section{SCATTER}

$S G S O(N, N)$

$\operatorname{SGSI}(N, N)$

SGSO $(N, N+1) \quad S G S I(N, N+1)$

$1.7851 E-01$

$1.9481 E-01$

$2.0755 E-01$

$1.9663 E-01$

$1.7247 F-01$

$7 \cdot 2415 E-03$

$7.8790 E-03$

$8.4135 E-03$

$8.0630 E-03$

$7.1942 E-03$

$2.0071 E-01$

$2.4300 E-01$

$2.5085 E-01$

$3.2326 E-01$

$5.7664 E-01$

$7.470>E-01$

$7.3197 E-01$

$7.432 .9 E-01$

$7.3408 E-01$

$7.2034 E-01$

$8.3294 E-03$

$1.0074 E-02$

$1.0473 E-02$

$6.4085 E-03$

$1.1205 \mathrm{E}-0$ ?

$1.4398 E-0$ ?

$1.4076 E-0 ?$

$1.4284 E-02$

$1.5746 E-0$ ?

$1.8711 E-02$

$1.4693 E-02-4.7569 E-03$

$1.5981 E-02-5.1769 E-03$

$1.7062 E-02-5.5284 E-03$

$1.6366 E-02-5 \cdot 2944 E-03$

$1.4610 E-02-4.7209 E-03$

$1.6916 E-02-5.46 .57 E-03$

2.0454E-0? $-6.6117 E-03$

$2.1279 E-02-6.8696 E-03$

$6.4067 E-03-2.0698 E-03$

1. $1202 E-02-3.6275 E-03$

$1.4394 E-02-4.6545 E-03$

$1.4070 E-02-4.5589 E-03$

$1.4281 E-02-4.6241 E-03$

$1.8941 E-02-6.1370 E-03$

$2.8264 E-02-9.1518 E-03$

$7.1238 E-01 \quad 1.8521 E-02$

$7.0585 E-01 \quad 1.8372 E-02$

$7.0834 E-01 \quad 1.7949 E-0 ?$

$7.3335 \mathrm{~F}-01 \quad 9.4018 \mathrm{E}-03$
$2.7975 E-02-9.0592 E-03$

$2.7749 E-02-8.9862 E-03$

$2.6370 E-0 ?-8.5378 E-03$ 


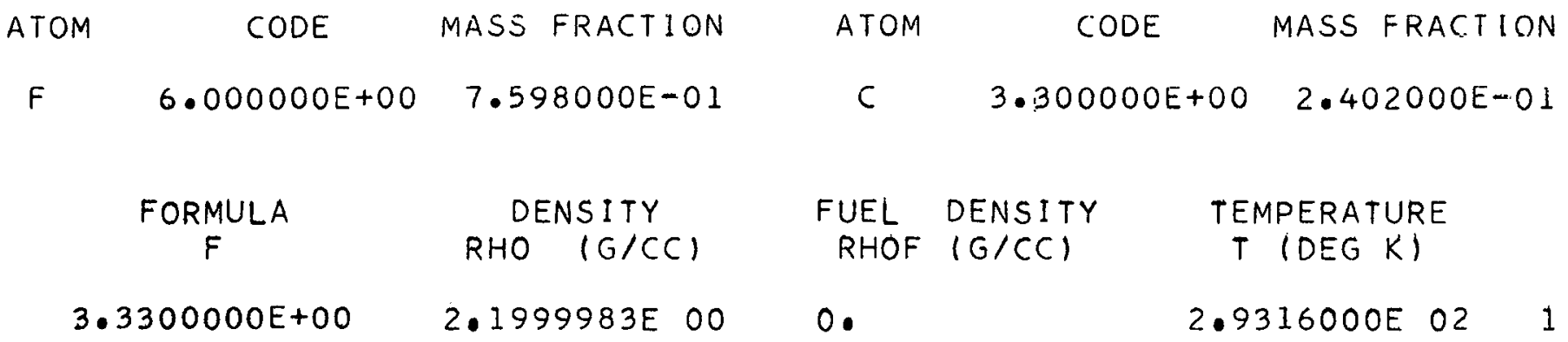

PROBABILITY/PATH (/CM)

$\begin{array}{cl}\text { COLLIDE } & \text { MULTIPLY } \\ \text { SG }(N) & \text { NUSGF }(N)\end{array}$

$9.4741 E-02$ $1.0799 E-01$ $1.4443 E-01$ $1.8220 E-01$ $2.0737 E-01$

$3.0569 E-01$ 4.7106E-01 $5.2513 E-01$ 4. $1607 E-01$ $3 \cdot 1801 E-01$ $3.0741 E-01$ $3.0477 E-01$ 3.0478E-01 $3.0614 E-01$ $3.0750 E-01$

$3.1022 E-01$ 3.1297E-01 $3.2436 E-01$ $3.2723 E-01$
0

0 .

0.

0.

0.

0.

0.

0.

0.

0.

0.

0.

0.

0 .

0.

0.

0 .

0.

0.

\section{PROBABILITY/PATH $Y / C M I$}

SGSO $(N, N)$ SGSI(N,N) SGSO $(N, N+1)$ SGSI $(N, N+1)$

7.105IE-02 1.1288E-02 2.3690E-02 -7.1558E-03

$8.1435 E-02 \quad 1.2665 E-02$

$1.0928 E-01 \quad 1.6773 E-02$

$1.3878 E-01 \quad 2.0741 E-02$

$1.5729 E-01 \quad 2.3904 E-02$

2.6557E-02 -8.0366E-03

$3.5151 E-02-1.0650 E-02$

4.3419E-02 -1.3186E-02

$5.0077 E-02-1.5185 E-02$

$2.3363 E-01$
$3.6362 E-01$

$3.4446 E-02$

$7.2065 E-02-2.1913 E-02$

4.0543E-01

5. $1451 E-02$

$5.7321 E-02$

$2.4651 E-02$

$1.9811 E-02$

$1.0744 E-01-3.2797 E-02$

$1.1970 E-01-3.6540 E-02$

$2.4658 E-02-7.4929 E-03$

$2.9819 E-01$

$1.9818 E-02-5.9880 E-03$

$2.8814 E-01$

2. $8562 E-01$

1.9268E-02

1. $9132 \mathrm{E}-02$

$2.8562 \mathrm{E}-01$

$1.9132 \mathrm{E}-02$

$1.9274 E-02-5.8200 E-03$

$1.9138 E-02-5.7780 E-03$

$1.9138 E-02-5.7780 E-03$

$2.5658 E-02-7.7454 E-03$

$2.8043 E-01 \quad 2.1174 E-02$

$2.5183 E-02$

$3.8698 E-02-1.1680 E-02$

2.7109E-01 2.5361E-02

$2.7347 E-01 \quad 2.5539 E-02$

2.8560E-01 2.5693E-0?

$3.2668 E-01 \quad 1.4118 E-02$
$3.8970 E-02-1.1764 E-02$

$3.9242 E-02-1.1848 E-02$

$3.8343 E-02-1.1583 E-02$ 
*THERMOFLEX

*THERMOFLEY.

ATOM CODE MASS FRACTION ATOM CODE MASG; HRACTION

$\begin{array}{llllll}\mathrm{O} & 3.600000 \mathrm{E}+00 & 4.942000 \mathrm{E}-\mathrm{OI} & \mathrm{FE} & 4.000000 \mathrm{E}+00 & 4.200000 \mathrm{E}-03 \\ \mathrm{SI} & 5.500000 \mathrm{E}+00 & 2.337000 \mathrm{E}-\mathrm{OI} & \mathrm{K} & 7.400000 \mathrm{E}+00 & 3.300000 \mathrm{~F}-03 \\ \mathrm{AL} & 5.10000 \mathrm{E}+00 & 2.223000 \mathrm{E}-\mathrm{OI} & \mathrm{CA} & 5.400000 \mathrm{E}+00 & 2.100000 \mathrm{E}-03\end{array}$

FORMULA
F

\author{
DENS ITY \\ RHO (G/CC)
}

$2.2399994 E-01$
FUEL DENSITY P.HOF $(\mathrm{G} / \mathrm{CC})$

TEMPERATURE

$T$ (DEG K)

0.

$2.9316000 E$ O2 1 ?

PROBABILITY/PATH (/CM)

\section{COLLIDE MULTIPLY $S G(N) \quad$ NUSGF $(N)$}

\section{$7.8496 \mathrm{E}-03$}

1. $.583 \mathrm{E}-0$ ?

1. $\angle 000 E-0$ ?

1. $5488 F-0$ ?

$2.4477 E-0$ ?

2. $9.9375-0$ ?

$2.4838 E-0$ ?

$2.6003 E-02$

$2.6288 E-02$

$2.1837 E-02$

$2.0202 E-02$

2. $0130 E-0$ ?

$2.0245 E-02$

$2.037 ? E-02$

?. $0597 \mathrm{E}-0$ ?

2.06>0E-0?

2. $0845 E-0$ ?

2.1901E-0?

$2.3049 E-02$
0.
0 .
0 .
0.
0 .

0.
0
0
0.

0.

0

0.

0.

0.

0

SCATTER

$S G S O(N \cdot N)$

$5.24375-03$

8.6831E-03

$9.3712 E-03$

$1.0637 E-0$ ?

1. 772 ? E-0?

1.9810E-0?

$1.7233 E-02$

2.0777E-02

$2.4998 E-02$

$2.0707 E-02$

1.9101E-0?

$1.90 ? 0 E-0 ?$

$1.9105 E-02$

1. $8817 \mathrm{E}-0$ ?

$1.81<6 F-02$

$1.8142 \mathrm{E}-0$ ?

$1.8182 F-0$ ?

$1.8966 E-02$

$2 \cdot 2084 E-02$

\begin{tabular}{|c|c|c|}
\hline \multicolumn{2}{|c|}{ SCATTER } & \multirow[b]{2}{*}{$\operatorname{SGS} 1(N, N+1)$} \\
\hline $\operatorname{SGSI}(N, N)$ & SGSO $(N, N+1)$ & \\
\hline $\begin{array}{l}9.7549 \mathrm{E}-04 \\
1.4964 \mathrm{E}-03 \\
1.6650 \mathrm{E}-03 \\
1.8059 \mathrm{E}-03 \\
2.8974 \mathrm{E}-03\end{array}$ & $\begin{array}{l}1.9208 E-03 \\
2.9394 E-03 \\
3.1806 E-03 \\
3.4986 E-03 \\
5.9202 E-03\end{array}$ & $\begin{array}{r}-6.0763 E-06 \\
-1.7847 E-04 \\
3.3042 E-05 \\
-1.1570 E-04 \\
-1.0723 E-03\end{array}$ \\
\hline $\begin{array}{l}3.5213 E-03 \\
3.0291 E-03 \\
2.5135 E-03 \\
1.2893 E-03 \\
1.1276 E-03\end{array}$ & $\begin{array}{l}6.9804 E-03 \\
6.0299 E-03 \\
5.2249 E-03 \\
1.2896 E-03 \\
1.1278 E-03\end{array}$ & $\begin{array}{l}-6.9051 E-04 \\
-6.2990 E-04 \\
-1.6101 E-03 \\
-3.9754 E-04 \\
-3.4533 E-04\end{array}$ \\
\hline $\begin{array}{l}1.0957 E-03 \\
1.0946 E-03 \\
1.0978 E-03 \\
1.2133 E-03 \\
1.4415 E-03\end{array}$ & $\begin{array}{l}1.0954 E-03 \\
1.0949 E-03 \\
1.0981 E-03 \\
1.4681 E-03 \\
2.2068 E-03\end{array}$ & $\begin{array}{l}-3.3580 E-04 \\
-3.3560 E-04 \\
-3.3662 E-04 \\
-4.5008 E-04 \\
-6.7660 E-04\end{array}$ \\
\hline $\begin{array}{l}1.4413 E-03 \\
1.4467 E-03 \\
1.4693 E-03 \\
8.3624 E-04\end{array}$ & $\begin{array}{l}2.2065 E-03 \\
2.2150 E-03 \\
2.1858 E-03\end{array}$ & $\begin{array}{l}-6.7650 E-04 \\
-6.7903 F-04 \\
-6.6992 E-04\end{array}$ \\
\hline
\end{tabular}

RELIABILITY LIMITATION: First-significant-figure uncertainty in high energy scatter cross sections of aluminum content. 
161

$\begin{array}{lllllllllllllllll}0 & 1 & 3 & 0 & 1 & 4 & 0 & 1 & 5 & 0 & 1 & 6 & 0 & 1 & 7 & 0 & 1\end{array}$ $5.3090 E-04 \quad 1.5409 E-04 \quad 0 . \quad 0$. $\begin{array}{llllllll}0 & 1 & 9 & 0 & 1 & 10 & 0\end{array}$

0

111

117

0.

0.25

$3.1775 E-04$

0 ? 11

0

0

217

7

026

$1.1866 E-04$

0.

218

$0.3{ }^{3} 8$

o 314

0 .

046

6.1256E-04

0412

0 .

0
0
0
0
0
0
0
0.

0.418

0.

0 .

0712

0.0718

$0.8 \% 14$

0.011

$\begin{array}{ccc}0.17 & 9 & 17\end{array}$

0 .

$0 \quad 10 \quad 15$

0 .

01114

0 .

ก 1?. 14

$0.013 \quad 15$

$\begin{array}{lll}0 & 14 & 17\end{array}$

0 .

01618

0 . $\begin{array}{lll}0 & 3 & 0\end{array}$

$1.1114 \mathrm{E}-04$

0315

0.07

$3.7154 E-0_{4}$

0413

0.

0

0

419

512

0.518

0.

0

612

0.018

$\begin{array}{ccc}C_{0} & 713 \\ 0 & & 18\end{array}$

0

0

719

0815

0.

0912

0.

918

0 .

01016

0 .

01115

0 .

$0.12 \quad 15$

$\begin{array}{lll}0 & 1316\end{array}$

0.

01418

001619
0

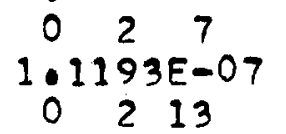

0.

219

0

0

0.

048

2.2535E-04

0.

- 57

$7.1711 F-04$
0

0.

C 519

0.

0

0.

0

0

0 :

613

619

714

810

0.

0816

0.

0913

0.

0

0.

$0 \quad 1017$

0.

01116

0.

01216

0.

$\begin{array}{lll}0 & 13 & 17\end{array}$

$0: 1419$

0.

01719
0. 112

$0: 18$

0.

028

$0: 214$

0.

$6.083 .6 E^{5}-04$

0311

0.

0

0.

049

$1.4352 E-04$

0 .

058

$1.0273 E-04$

0514

0.

068

1.0183E-03

0614

0 .

079

$1.5748 E-03$

0715

$0:$

0

0

811

0817

0.014

0 .

01012

0.

01018

0.

01117

0 .

$0 \quad 1217$

0.

01318

0.

$\begin{array}{lll}0 & 1517\end{array}$

0 . o. 113

$0: 019$

0.

029

0015

0.

036

3. $6899 E-04$

0312

0 .

0318

0.

0

410

0

0 .

426

059

0

0.

5. 15

069

$9.2898 E-04$

0615

0.

710

0.

0716

0 .

0

812

0

- 918

0.015

0.15

01013

0 .

01019

0 .

0

0.

01218

0.

01319

0.

01518

0 .
0114

$0: 24$

$5 \cdot 2388 E-04$

0210

$0: 216$

0.07

2.238OE-04

0313

0 .

0319

00411

0 .

0417

$0 \quad 510$

0.

0516

0.

0610

0616

0711

0.

$\begin{array}{lll}0 & 7 & 17\end{array}$

0813

0.819

$\begin{array}{lll}0.0 & 9 & 16\end{array}$

0.01014

0.

01113

0.

$0 \quad 1119$

0 .

$0121 \%$

0.

01416

0 .

01519 
$\begin{array}{llllllllllllllllll}1 & 1 & 3 & 1 & 1 & 4 & 1 & 1 & 5 & 1 & 1 & 6 & 1 & 1 & 7 & 1 & 1 & 8\end{array}$

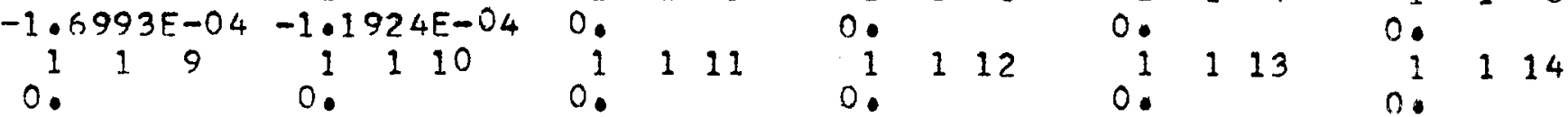

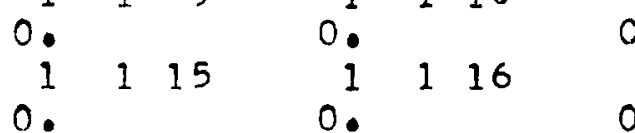
126 $1 ? 5$ 1711 0.1212 0 . 1 i ? 11 1218 i. 30 . 139

i

146

$-7.9002 E-05$

0.

$\begin{array}{lll}1 & 1 & 17\end{array}$

0.127 $12{ }^{7}$

1118

0 i 28

0 . 1213 0.

$1.759 ? 2-04-10$ 1.315 147 0 1316 1214 0.

135 148

$0.119 \quad 0124$

$0 . \quad 2.5089 E-05$

$\begin{array}{lllll}1 & 2 & 1 & 2 & 10\end{array}$

$1215 \quad 1216$

136 I. 37

$$
\text { i. }
$$

$\begin{array}{lll}1 & 4 & 12\end{array}$

$-1.7903 E-05$

$-9.7508 E-05-1.0792 E-04$

$1418 \quad 0: 419$

0

1511

0

1611

0

$\begin{array}{lll}1 & 6 & 17\end{array}$

$\begin{array}{lll}0 . & & 19 \\ 1 & 5 & 12\end{array}$ 0.

0.0 .

1757

$\begin{array}{ccc}-3 \cdot 1801 E-04 & -8.6285 E-05 \\ 1513 & 1 & 5\end{array}$

1518

0.519

1

1612

0.18

$0 ; 71 ?$

$0: 713$

0.718

0.719

$0: \begin{array}{lll}1 & 7 & 18 \\ 0 & 8 & 14\end{array}$

0719

$\begin{array}{lll}1 & 814 \\ 0 & & 14\end{array}$

$\begin{array}{lll}1 & 9 & 11\end{array}$

$\begin{array}{lll}1 & 9 & 17\end{array}$

i $10 \quad 15$

0 .

11114

0.

]. 1714

0.1315

0 .

$\begin{array}{lll}1 & 14 & 17\end{array}$

1815

0 i 912

0.

1918

0. 1016

1

1619

$0: 714$

0.810

1816

0.

1913

$-4.7229 E-04$

0.

1919

$0: 11017$

i 1115

0 .

0. 1215

$c$

0.

1 $16 \quad 18$

0.

0.

0 .
11316

11418

11619

11116

$0: 1216$

0.1317

0.

11419

0.

$\begin{array}{lll}1 \quad 17 & 19\end{array}$ $2.5038 E-04$ 1311 $\begin{array}{ll}0 . & 317 \\ 0 . & \end{array}$$$
0
$$

0.514

168

$9.9990 E-05$

1614

179

i 811

$0:-11$

$0: 17$

0.

11012

$5.4514 E-05$

1312

0.

0 .

0

1

318

416

159

0.

515

0.

169

$-4.5485 E-04$

0! 615

1710

$0: 716$

$\begin{array}{lll}0 . & & \\ 1 & 8 & 12\end{array}$

$0: 812$

01818

1

11013

0.1018

0 .

0.1117

11019

0.1118

$0: 1217$

$0: 1217$

0 .

11319

0.1517

0.

11518

0 .
410

0 i 1218
3
$-2.3903 E-05$

1313

0.

1319

1411

0.

1417

0.

1510

1516

$0 \in 10$

0 .

1616

0.

1711

$\begin{array}{lll}0 & 7 & 17\end{array}$

$\begin{array}{lll}0 . & 7 & 17\end{array}$

0.

1819

0.

1916

0.

11014

0.

11113

0 .

11119

$0: 1219$

0.

11416

0 .

11519 


\begin{tabular}{|c|c|c|c|c|c|}
\hline \multicolumn{2}{|c|}{ CODE } & MASS FRACTION & \multicolumn{2}{|l|}{ ATOM } & MASS FRACTION \\
\hline \multicolumn{2}{|c|}{$8 \cdot 500000 E+00$} & $9.387000 E-01$ & \multicolumn{2}{|c|}{$3.300000 E+00$} & $6.130000 E-02$ \\
\hline \multicolumn{2}{|c|}{$\begin{array}{c}\text { FORMULA } \\
F\end{array}$} & $\begin{array}{l}\text { DENSITY } \\
\text { HO }(G / C C)\end{array}$ & $\begin{array}{l}\text { FUEL DENS } \\
\text { RHOF } 1 G / C\end{array}$ & $\begin{array}{l}\text { DENSITY } \\
(G / C C)\end{array}$ & $\begin{array}{l}\text { TEMPERATURE } \\
T(D E G K)\end{array}$ \\
\hline \multicolumn{2}{|c|}{$8.5300000 E+00$} & $1.5630006 \mathrm{E} \quad 01$ & 0 & \multirow[t]{2}{*}{2.931} & $6000 E \quad 02$ \\
\hline \multicolumn{5}{|c|}{ PROBABILITY/PATH (/CM) } & \\
\hline $\begin{array}{l}\text { COLLIDE } \\
\text { SG }(N)\end{array}$ & 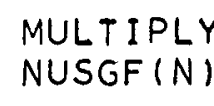 & $S G S O(N, N)$ & $\begin{array}{l}S C A \\
S G S I(N, N)\end{array}$ & $\begin{array}{l}\text { TTER } \\
\operatorname{SGSO}(N, N+1)\end{array}$ & SGS1 $(N, N+1)$ \\
\hline $\begin{array}{l}3.1123 E-01 \\
3.4969 E-01 \\
4.0618 E-01 \\
4.2903 E-01 \\
4.5308 E-01\end{array}$ & $\begin{array}{l}0 \\
0 . \\
0 \\
0 \\
0\end{array}$ & $\begin{array}{l}2.8404 E-01 \\
3.2093 E-01 \\
3.7082 E-01 \\
3.9065 E-01 \\
4.0703 E-01\end{array}$ & $\begin{array}{l}1.2966 E-02 \\
1.3751 E-02 \\
1.6842 E-02 \\
1.8342 E-02 \\
2.1940 E-02\end{array}$ & $\begin{array}{l}2.7136 E-02 \\
2.8697 E-02 \\
3.5276 E-02 \\
3.8268 E-02 \\
4.5906 E-02\end{array}$ & $\begin{array}{l}-8.2337 E-03 \\
-8.7505 E-03 \\
-1.0691 E-02 \\
-1.1666 E-02 \\
-1.3919 E-02\end{array}$ \\
\hline $\begin{array}{l}4.9204 E-01 \\
5.3726 E-01 \\
5.8250 E-01 \\
6.5660 E-01 \\
8.0425 E-01\end{array}$ & $\begin{array}{l}0 . \\
0 . \\
0 . \\
0 .\end{array}$ & $\begin{array}{l}4.3643 E-01 \\
4.7363 E-01 \\
5.1226 E-01 \\
6.3653 E-01 \\
7.8142 E-01\end{array}$ & $\begin{array}{l}2.6410 E-02 \\
3.0162 E-02 \\
3.3327 E-02 \\
1.9460 E-02 \\
2.1147 E-02\end{array}$ & $\begin{array}{l}5.5437 E-02 \\
6.3400 E-02 \\
6.9942 E-02 \\
1.9439 E-02 \\
2.1138 E-02\end{array}$ & $\begin{array}{l}-1.6712 E-02 \\
-1.9066 E-02 \\
-2.1084 E-02 \\
-5.8503 E-03 \\
-6.3668 E-03\end{array}$ \\
\hline 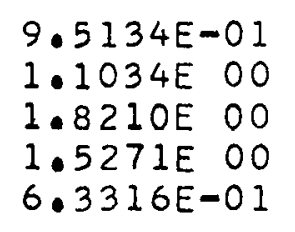 & $\begin{array}{l}0 . \\
0 . \\
0 . \\
0 . \\
0 .\end{array}$ & $\begin{array}{l}9.2480 E-01 \\
1.0682 E \quad 00 \\
8.2921 E-01 \\
4.8551 E-01 \\
4.5063 E-01\end{array}$ & $\begin{array}{l}2.1972 E-02 \\
2.2783 E-02 \\
2.1421 E-02 \\
2.1674 E-02 \\
2.5661 E-02\end{array}$ & $\begin{array}{l}2.1935 E-02 \\
2.2733 E-02 \\
2.1404 E-02 \\
2.6277 E-02 \\
3.9531 E-02\end{array}$ & $\begin{array}{l}-6.6581 E-03 \\
-6.9356 E-03 \\
-6.4633 E-03 \\
-7.8444 E-03 \\
-1.1786 E-02\end{array}$ \\
\hline $\begin{array}{l}7.0647 E-01 \\
8.5789 E-01 \\
1.1337 E 00 \\
1.2161 E 00\end{array}$ & $\begin{array}{l}0 . \\
0 . \\
0 . \\
0 .\end{array}$ & $\begin{array}{l}4.3161 E-01 \\
4.3161 E-01 \\
4.3533 E-01 \\
4.0605 E-01\end{array}$ & $\begin{array}{l}2.5540 E-02 \\
2.5540 E-02 \\
2.5125 E-02 \\
1.3255 E-02\end{array}$ & $\begin{array}{l}3.9319 E-02 \\
3.9319 E-02 \\
3.7562 E-02\end{array}$ & $\begin{array}{l}-1 \cdot 1736 E-02 \\
-1 \cdot 1736 E-02 \\
-1.1211 E-02\end{array}$ \\
\hline
\end{tabular}




\begin{tabular}{|c|c|c|c|c|c|c|}
\hline ATOM & CODE & MASS FRACTION & ATOM & $\mathrm{CO}$ & & MASS FRACTION \\
\hline $\mathrm{H}$ & $2.040000 E+00$ & $1.119020 E-01$ & 0 & 3.6000 & +00 & $8.880980 E-0 I$ \\
\hline & $\begin{array}{c}\text { FORMULA } \\
F\end{array}$ & $\begin{array}{c}\text { DENSITY } \\
\text { RHO }(G / C C)\end{array}$ & $\begin{array}{l}\text { FUEL } \\
\text { RHOF }\end{array}$ & $\begin{array}{l}\text { DENSITY } \\
\text { (G/CC) }\end{array}$ & $\underset{T}{T E M}$ & $\begin{array}{l}\text { PPERATURE } \\
\text { (DEG K) }\end{array}$ \\
\hline 2 & $100000 E+00$ & $9.9799999 E-01$ & 0. & & 2.93 & $316000 E 02101$ \\
\hline
\end{tabular}

PROBABILITY/PATH (/CM)

\begin{tabular}{|c|c|}
\hline $\begin{array}{l}\text { COLLIDE } \\
\text { SG(N) }\end{array}$ & \\
\hline $\begin{array}{l}9.9451 E-02 \\
1.6853 E-01 \\
2.1759 E-01 \\
2.8300 E-01 \\
4.2099 E-01\end{array}$ & \\
\hline $\begin{array}{l}5.2344 \mathrm{E}-01 \\
6.0655 \mathrm{E}-01 \\
7.2921 \mathrm{E}-01 \\
1.0563 \mathrm{E} O 0 \\
1.3750 \mathrm{E} O 0\end{array}$ & $\begin{array}{l}1 \\
1 \\
1 \\
0 \\
0\end{array}$ \\
\hline $\begin{array}{ll}1.4452 \mathrm{E} & 00 \\
1.4604 \mathrm{E} & 00 \\
1.4609 \mathrm{E} & 00 \\
1.4785 \mathrm{E} & 00 \\
1.5001 \mathrm{E} & 00\end{array}$ & $\begin{array}{l}0 \\
0 \\
0 \\
0 \\
0\end{array}$ \\
\hline $\begin{array}{ll}1.6658 \mathrm{E} & 00 \\
2.1275 \mathrm{E} & 00 \\
2.7740 \mathrm{E} & 00 \\
3.1781 \mathrm{E} & 00\end{array}$ & $\begin{array}{l}0 \\
0 \\
0 \\
0\end{array}$ \\
\hline
\end{tabular}

SCATTER

SGSO $(N, N) \quad$ SGSI $(N, N) \quad \operatorname{SGSO}(N, N+1) \quad \operatorname{SGS} 1(N, N+1)$

$3.3559 E-02 \quad 1.7798 E-02$

$6.5922 E-02 \quad 2.8799 E-02$

$7.6739 E-02 \quad 3.8442 E-02$

$9.4495 E-02 \quad 5.0797 E-02$

$1.6273 E-01$

$7.2231 E-02$

$1.9675 E-01$

$1.9972 E-01$

$2.2358 E-01$

$6.4614 E-01$

$8.2707 E-01$

$8.6686 E-01$

$8.7538 E-01$

$8.7538 E-01$

$7.6666 E-01$

$6.1473 E-01$

$6.7764 E-01$

$8.6264 E-01$

$1.2428 E$ OO

$3.1586 \mathrm{E} \quad 00$

$9.0650 E-02$

1.0930E-01

$1.3388 E-01$

4.3179E-01

5.7700E-OI

$6.0893 E-01$

$6.1578 E-01$

$6.1548 E-01$

5.5116E-01

4. $4865 E-01$

4. $9342 E-01$

6.2646E-01

$8.2350 E-01$

$9.0828 \mathrm{E}-01$
$2.9253 E-02$

4. $8444 E-02$

$6.3589 E-02$

$8.3367 E-02$

1. $2126 \mathrm{E}-01$

1.7330E-02

$2.4033 E-02$

$3.5961 \mathrm{E}-02$

4. $9899 \mathrm{E}-02$

$6.1171 E-02$
$1.5146 E-01 \quad 7.9349 E-02$

$1.7903 E-01 \quad 1.0860 E-01$

4.4128E-01 2.6013E-01

$3.5565 E-01 \quad 1.8471 E-01$

$4.7479 E-01 \quad 2.4872 E-01$

5.0099E-01 2.6280E-01

5.0661E-01 2.6581E-01

4.5594E-01 2.4958E-01

4.5260E-Ol 2.7527E-01

$5.6334 E-01 \quad 3.5616 E-01$

$6.3167 E-01 \quad 3.6787 E-01$

$8.5738 E-01$ 4.1315E-0I

$1.5160 E$ OO $6.6134 E-02$ 
013

$1.4416 E-02$

019

1. $5772 E-03$

0115

$-7.3205 E-08$

025

1.2926E-02

0211

$7.0368 E-05$

0217

0.

$\begin{array}{lll}0 & 3 & 8\end{array}$

$6.7830 E-03$ 0314

4.2451E-07 046

4. $1368 E-02$

0412

$5.0311 E-05$

0418

0 .

$0 \quad 511$

7. $9811 E-04$

$0 \quad 517$

$3.4216 E-07$

0611

$1.6832 E-03$

0617

$8.7528 E-08$

0712

4. $8826 E-04$ 0718

$1.1379 E-07$

0814

$1.6851 E-05$

0911

4.7108E-02

0917

$2.6085 E-06$

01015

1. $8897 \mathrm{E}-04$

01114

$8.1199 E-03$

01214

$6.0681 E-02$

01315

$8.1405 E-02$

$0 \quad 14 \quad 17$

$5.9888 E-02$

$016 \quad 1.8$

2. $3389 E-01$
014

$8.7438 E-03$

0110

$2.1346 E-04$

0116

0.

026

$7.8398 E-03$

0212

$9.5501 E-06$

0218

0 .

039

$9.0408 E-03$

0315

7. $7184 E-08$

047

2.5091E-02

0413

$6.8797 E-06$

0419

0 .

0512

$1.0809 E-04$

$0 \quad 518$

$-2.7372 E-07$

0612

$2.2775 E-04$

0618

0 .

0713

$6.6224 E-05$

0719

$0 \quad 8 \quad 15$

$2.8805 E-06$

0912

$6.3755 E-03$

0918

$9.4351 \mathrm{E}-07$

01016

$6.9619 E-05$

01115

$1.4742 E-03$

01215

$1.1017 \mathrm{E}-02$

01316

$2.9947 E-02$

01418

$2.2718 E-02$

01619

$1.1715 \mathrm{E}-01$
015

$5.3034 E-03$

0111

$2.8907 E-05$

0117

0.

0
4

4. $7550 E-03$

0213

1.3257E-06

0219

0.

0310

$1.2236 E-03$

0316

0.

048

1. $5218 \mathrm{E}-02$

0414

$7.3524 E-07$

057

$5.3904 E-02$

0513

$1.4576 \mathrm{E}-05$

0519

0.

0613

$3.0635 E-05$

O 619

0.

0714

$7.8513 E-06$

0810

5. 5628E-02

0816

$1.0082 E-06$

0913

$8.6270 E-04$

0919

4.4401E-07

$0 \quad 10 \quad 17$

2.5627E-05

01116

$5.4216 E-04$

01216

4.0530E-03

O 1317

1. $1017 \mathrm{E}-02$

O 1419

1.2135E-02

$0 \quad 1719$

$3.9839 E-01$ $\begin{array}{lllllllll}0 & 1 & 6 & 0 & 1 & 7 & 0 & 1 & 8\end{array}$

$3.2167 E-03$

$\begin{array}{lll}0 & 1 & 12\end{array}$

$3.8982 E-06$

0118

0.

028

$2.8841 E-03$

0214

$1.0822 \mathrm{E}-07$

035

$3.0399 E-02$

0311

$1.6558 E-04$

0317

0 .

049

$2.0284 E-02$

0415

I. 5755E-07

058

$3.2694 E-02$

0514

$1.7792 \mathrm{E}-06$

068

$6.8946 E-02$

0614

3. 9388 E-06

079

$1.9697 E-01$

0715

$1.5930 E-06$

0811

$7.5284 E-03$

0817

4.3207E-07

0914

$1.0495 E-04$

$0 \quad 1012$

$6.3233 E-02$

01018

9.6103E-06

01117

1.9952E-04

$0 \quad 1217$

$1.4910 E-03$

01318

4. $1793 E-03$

$\begin{array}{lll}0 & 15 & 17\end{array}$

$2.0168 E-01$
1.9510E-03 1.1833E-03

0113

$5.3074 E-07$

0114

0119

0.

029

3.844IE-03

0215

$5.4108 E-08$

036

$1.8438 E-02$

0312

2.2461E-05

0318

0.

0410

$2.7452 E-03$

$\begin{array}{ccc}0416 & 0 & 417 \\ 2.6258 E-07 & -1.5755 E-07\end{array}$

059

4.3577E-02

0515

$3.4216 E-07$

069

$9.1897 E-02$

0615

$7.0022 E-07$

0710

2.6656E-02

0716

$5.6893 E-07$

0812

$1.0188 \mathrm{E}-03$

0818

1.9092E-05

01013

$8.5576 E-03$

01019

$5.0659 E-06$

01118

$7.5765 E-05$

01218

5.6543E-04

01319

$2.2320 E-03$

01518

$7.6506 \mathrm{E}-02$

0510

$5.8975 E-03$

0516

$6.8431 E-08$

0610

$1.2437 E-02$

0616

$2.6258 E-07$

0711

$3.6076 E-03$

0717

0813

$1.3805 E-04$

0819

$-4.3207 E-07$

0916

$6.9931 E-06$

01014

1. $0404 \mathrm{E}-03$

01113

6.6779E-02

01119

4.0361E-05

01219

3.0213E-04

01416

$1.6279 E-01$
7.2012E-07 
113

$8.9808 E-03$

119

$1.8751 E-04$

1115

$3.2291 E-07$

125

$6.2763 E-03$

1211

$3.3582 E-06$

1217

0.

138

2.0030E-03

1314

$1.2875 E-07$

146

$2.5762 E-02$

1412

$2.1371 E-06$

1418

0.

1511

$3.9966 E-05$

1517

$-1.8545 E-07$

1611

$1.0122 E-04$

1617

$5.8389 E-08$

1712

$1.9585 E-05$

1718

$3.0364 \mathrm{E}-07$

1814

$1.4412 \mathrm{E}-06$

1911

9.3870E-03

1917

4.8132E-07

11015

$7.4547 E-06$

11114

6.5705E-04

$112 \quad 14$

$1.2658 E-02$

11315

$2.2947 E-02$

$1 \quad 14 \quad 17$

$1.3858 E-02$

11618

$5.5516 E-02-2.3794 E-02$ $\stackrel{1}{1} \stackrel{1}{4} \stackrel{4}{4.2461 E-03}$

1110

$1.1909 E-05$

1116

0.126

$2.9689 E-03$

1212

$8.3051 E-07$

1218

0.

139

$1.7172 E-03$

1315

5. $1498 E-08$

147

1.2176 E-02

1413

$5.2553 E-07$

1419

0.512

4.1313E-06

1518

$3.2240 E-07$

1612

$8.4080 E-06$

1618

$1.7517 \mathrm{E}-07$

1713

$3.3021 E-06$

1715

0.

$3.8433 E-07$

1912

4.9540E-04

1918

$3.3322 E-07$

11016

3.6280 E-06

11115

7.0304E-05

11215

$1.1863 E-03$

11316

5. $0646 E-03$

11418

3. $1624 E-03$

11619

115

$2.0087 E-03$

1111

$1.5451 E-06$

1117

0.

127

$1.4057 E-03$

1213

2.5277E-07

1219

0.

1310

$9.2285 E-05$

1316

$1.2875 E-07$

148

$5.7571 E-03$

1414

$2.4525 E-07$

157

$3.3568 E-02$

1513

9.1300E-07

1519

1613

$1.7517 E-06$

1619

0.

1714

$7.5911 E-07$

1810

$1.3484 \mathrm{E}-02$

1816

$1.9216 E-07$

1913

$3.4988 E-05$

1919

$6.2941 E-07$

11017

$1.7394 \mathrm{E}-06$

11116

$2.0942 E-05$

11216

$2.7908 E-04$

$113 \quad 17$

$1.0963 E-03$

11419

8. $1760 E-04$

11719

$-6.9789 E-02$
116

117

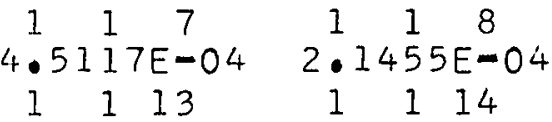

1112

4.397IE-07

1118

0.

128

$6.6660 E-04$

1214

$7.2219 E-08$

135

$1.8934 E-02$

1311

7.1068E-06

$\begin{array}{lll}1 & 3 & 17\end{array}$

0. 49

4. $9166 E-03$

1415

$3.5035 E-08$

158

$1.5865 E-02$

1514

$2.7390 E-07$

168

4. $2936 E-02$

1614

$3.5034 \mathrm{E}-07$

179

$1.0058 E-01$

1715

$5.3138 E-07$

1811

6.9866E-04

1817

$2.8825 E-07$

1914

4.7576E-06

$110 \quad 12$

$1.2602 E-02$

11018

$1.1431 \mathrm{E}-06$

$\begin{array}{lll}1 & 11 & 17\end{array}$

$7.9252 E-06$

$\begin{array}{lll}1 & 12 & 17\end{array}$

$7.0997 E-05$

11318

$2.2865 E-04$

$\begin{array}{lll}1 & 15 & 17\end{array}$

7.5601E-02

$1.4657 E-07-2.0077 E-07$

1119

129

5.7606E-04

1215

$1.2638 \mathrm{E}-07$

136

$8.9499 E-03$

1312

$1.2617 \mathrm{E}-06$

1318

0.1410

$2.5458 E-04$

1416

$-2.3211 E-07$

159

$1.3526 E-02$

1515

$9.1300 E-08$

169

$3.6574 E-02$

1615

$2.3356 E-07$

1710

$5.0538 E-03$

1716

$7.5911 E-08$

1812

$4.4870 E-05$

1818

$-6.5456 E-07$

1915

$1.3699 \mathrm{E}-06$

$110 \quad 13$

$6.6563 E-04$

11019

$1.9382 E-06$

11118

$3.8839 E-06$

I 1218

$2.1684 E-05$

11319

$1.9795 E-05$

11518

$1 \cdot 3275 E-02$.

1210

$3.3311 E-05$

1216

0.

$\begin{array}{lll}1 & 3\end{array}$

4. $2325 E-03$

1313

$3.8624 E-07$

1319

0.

1411

$1.6326 E-05$

1417

4.0728E-07

1510

$6.8885 E-04$

1516

9.1300E-08

1610

$1.8461 E-03$

1616

$5.8389 E-08$

1711

2.6859E-04

1717

$7.5911 E-08$

1813

5.9090E-06

1819

$1.0389 E-06$

1916

$8.1454 \mathrm{E}-07$

$\begin{array}{lll}1 & 10 & 14\end{array}$

4.264IE-05

11113

$1.3309 E-02$

11119

$5.0386 E-06$

11219

$1.6270 E-05$ 
ATOM

CODE

MASS FRACTION

D

$3 \cdot 100000 E+00$

$2.011327 E-01$

FORMULA

$F$

DENSITY

RHO (G/CC)
ATOM

0

FUEL DENSITY

RHOF (G/CC)
CODE

MASS FRACTION

0 .

\section{$3.600000 E+00$}

$7.988674 E-01$
$3.1100000 E+00$
PROBABILITY/PATH $(/ \mathrm{CM})$
SCATTER

SGSO $(N, N) \quad \operatorname{SGSI}(N, N) \quad \operatorname{SGSO}(N, N+I) \quad \operatorname{SGSI}(N, N+I)$

$3.6199 E-02 \quad 1.8774 E-02$

6.8138E-02 2.8791E-02

$7.7370 E-02 \quad 3.6294 E-02$

9.0855E-02 4.4054E-02

$1.5135 \mathrm{E}-01 \quad 5.7755 \mathrm{E}-02$

$1.7177 E-01$

1. $5180 E-01$

$1.4864 E-01$

$2.6061 E-0.1$

$2.6061 E-01$

$6.3275 E-02$

$6.0621 E-02$

$6.0138 E-02$

$8.7057 E-02$

$8.7057 E-02$

$2.6061 E-01$

$2.6061 E-01$

2.6061E-01

$2.3655 E-01$

$2.0275 E-01$

$8.7057 E-02$

$8.7057 E-02$

$8.7057 \mathrm{E}-02$

$8.5411 E-02$

$7.7634 \mathrm{E}-02$

2.0759E-01

2.2076E-0I

$2.4926 E-01$

4.6644E-01

$3.3063 E-02$

$5.1599 E-02$

$6.4441 E-02$

$7.8003 E-02$

$1.0455 \mathrm{E}-01$

]. $1497 \mathrm{E}-01$

$1.0924 E-01$

$1.9931 E-01$

$8.8233 E-02$

$8.8233 E-02$

2

$8.8233 E-02-6.7910 E-03$

$8.8233 E-02-6.7910 E-03$

$8.4149 E-02-3.7883 E-03$

$9.1751 E-02 \quad 5.9454 E-03$

$1.1573 E-01$ 1.8846E-02

8. $1173 E-02$

$9.0341 E-02$

$1.0522 \mathrm{E}-01$

$1.1663 E-01$
$1.2098 E-01 \cdot 2.0072 E-02$

$1.3831 E-01 \quad 2.2066 E-02$

$1.9028 E-01 \quad 2.7977 E-03$
1.3969E-02

$2.4799 E-02$

3. $1014 E-02$

$3.2228 E-02$

$2.0970 E-02$

$-6.7910 E-03$ 
013

$1.6722 E-02$

01

0.115

0 .

025

$1.4080 E-02$

0211

0 .

$\begin{array}{lll}0 & 2 & 1.7\end{array}$

0.03

$7.8749 E-04$

0314

0.06

3.8101E-02

0412

0 .

0418

0.011

$\begin{array}{lll}0.17 & 5 & 17\end{array}$

0.011

0.017

0.712

0 .

0718

$\begin{array}{ccc}0.0 & & \\ 0 & 8 & 14\end{array}$

0.911

$3.0115 E-04$

0917

0 .

01015

0.1114

0 .

O 1214

$3.0115 E-04$

01315

4. $3849 \mathrm{E}-03$

$014 \quad 17$

4.0154E-04

01618

$3.1946 E-02$
014

$1.0143 E-02$

0110

0.16

0 .

026

$7.4296 E-03$

0212

$0 .: 218$

0.039

0.

0315

0.7

2.3110E-02

0413

0 .

0419

0 .

$0 \quad 512$

$\begin{array}{lll}0 & & \\ 0 & 5 & 18\end{array}$

$\begin{array}{lll}0 & 0 & \\ 0 & 12\end{array}$

$\begin{array}{lll}0 & 0 & 18\end{array}$

$\begin{array}{lll}0 . & & 18 \\ 0 & 7 & 13\end{array}$

0 .

0719

$\begin{array}{lll}0 & 0 & \\ 0 & 8 & 15\end{array}$

$\begin{array}{ccc}0 & 0 & 12\end{array}$

0

0918

0.1016

0.01115

c.

01215

$0: 013 \quad 16$

0 .

01418

0 .

$0 \quad 1619$

$3.2781 E-04$ $\begin{array}{lll}0 & 1 & 5\end{array}$

$5.3520 E-0$

0111

$\begin{array}{lll}0 & 1 & 17\end{array}$

0 .

027

5.9152E-04

0213

0.

0219

0 .

0310

$\begin{array}{lll}0.16 & 3 & 16\end{array}$

0 .

048

1.2195E-02

0414

0.

057

4. 3746E-02

$0 \quad 513$

0.1919

0.013

$\begin{array}{lll}0 & 6 & 19\end{array}$

0 .

0714

0 .

0810

$1.2046 E-03$

0816

0.

0913

$\begin{array}{rrr}0 & 9 & 19\end{array}$

0.

$\begin{array}{lll}0 & 10 & 17\end{array}$

0.01116

0 .

01216

0 .

$0 \quad 1317$

0 .

01419

0.

$0 \quad 17 \quad 19$

$3.2673 E-02$ $\begin{array}{lllllllll}0 & 1 & 6 & 0 & 1 & 7 & 0 & 1 & 8\end{array}$

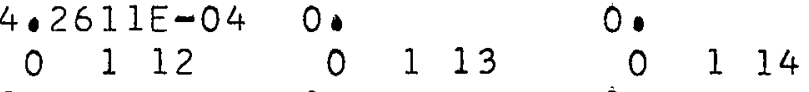

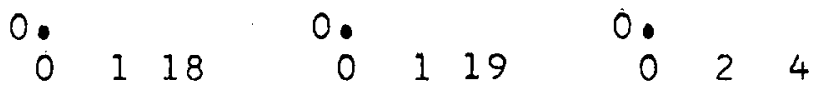
$0 . \quad 0.0214 \mathrm{E}-02$

$\begin{array}{lllllllll}0 & 2 & 8 & 0 & 2 & 9 & 0 & 2 & 10\end{array}$

0.

0214

0.

035

$3.0905 E-02$

0311

0.015

0 .

0216

0.0 .

$\begin{array}{llllll}0 & 3 & 6 & 0 & 3 & 7\end{array}$

0.0318

0313

$\begin{array}{lll}0 & 3 & 17\end{array}$

0 .

049

$9.7089 E-04$

0

410

$0 \quad 319$

$0415 \quad 0$

0

416

0411

$0 . \quad 0$.

059

$1.5116 E-02$

$0 \quad 515$

0 .

0.0

069

4.6780E-02

0614

0.079

$9.2282 E-02$

0715

$4.4538 E-02$

0615

0.710

0 .

0417

0 .

0510

0 .

0516

0 .

0610

0 .

0616

0.811

0.716

0 .

$\begin{array}{lll}0 & 7 & 17\end{array}$

$\begin{array}{rrr}0 . & & \\ 0 & 8 & 11\end{array}$

0 .

$0 \quad 812$

0 .

$0 \quad 813$

0.

$\begin{array}{lll}0 & 8 & 17\end{array}$

0.

0818

0.

$0 \quad 819$

$\begin{array}{rrr}0 . & & \\ 0 & 9 & 14\end{array}$

$\begin{array}{lll}0 & 0 & \\ 0 & 9 & 15\end{array}$

0 .

0916

$\begin{array}{lll}0 & 10 \quad 12\end{array}$

0 .

01013

0 .

01014

$3.0115 E-04$

01018

0 .

01019

0 .

01113

0 .

$\begin{array}{lll}0 & 11 & 17\end{array}$

0 .

01118

3.0115E-04

0 .

0 .

$\begin{array}{lll}0 & 12 & 17\end{array}$

01218

0 .

01318

0 .

0 .

$0 \quad 1319$

01119

0 .

01219

0.

01416

0.

?.0448E-0?

01517

01518

01519 


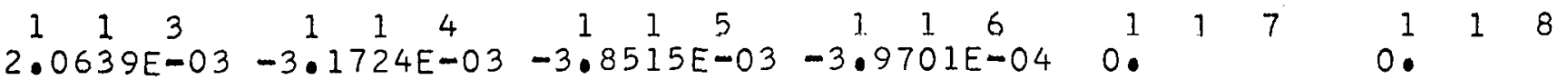
$\begin{array}{llllllllllllllllll}1 & 1 & 9 & 1 & 1 & 10 & 1 & 1 & 11 & 1 & 1 & 12 & 1 & 1 & 13 & 1 & 1 & 14\end{array}$

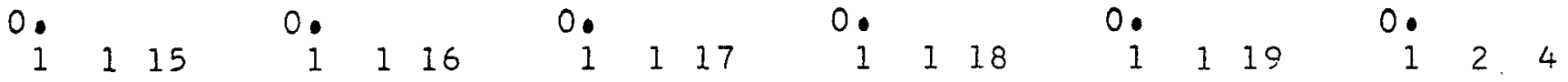
$0.0000000031 E-03$ $\begin{array}{lllllllllllllllll}2 & 5 & 1 & 2 & 6 & 1 & 2 & 7 & 1 & 2 & 8 & 1 & 2 & 9 & 1 & 2 & 10\end{array}$ $\begin{array}{cccccccccccc}-4.4039 E-03 & -5.3466 E-03 & -5.5112 E-04 & 0 . & & 0 . & & 0 . & 16\end{array}$

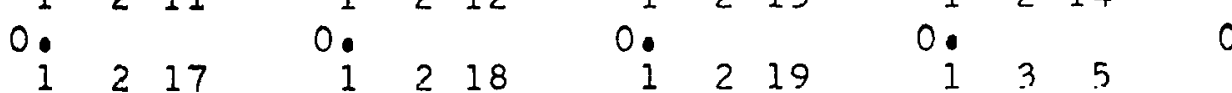
$\begin{array}{llllllllll}1 & 2 & 17 & 1 & 2 & 18 & 1 & 2 & 19 \\ 0 . & & & 0 & & & 0 & \end{array}$ $\begin{array}{llllllllll}1 & 3 & 8 & 1 & 3 & 9 & 1 & 3 & 10\end{array}$ $\begin{array}{lllllllll}1 & 3 & 5 & 1 & 3 & 6 & 1 & 3 & 7\end{array}$ $-7.3371 E-04 \quad 0$ 1314

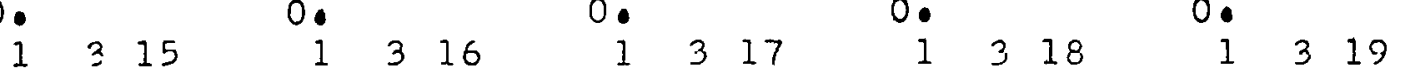

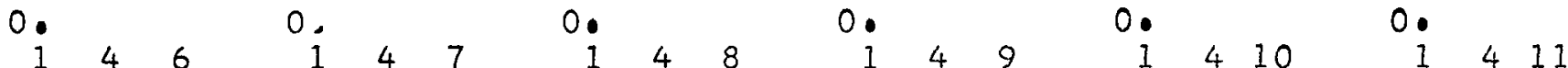
$4.7026 E-03-7.2282 E-03-8.7756 E-03-9.0457 E-04 \quad 0$

142214

0 .

$1418 \quad 1 \quad 4 \quad 19$

D. 511

0 .

1

0 .

$\begin{array}{llll}1 & 6 & 11\end{array}$

$0_{i}$

0.

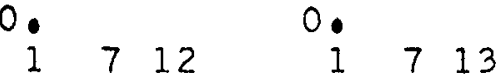

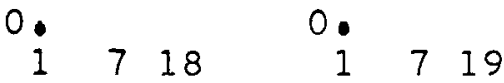

0 :

1

0.

1911

$-2.8059 E-04$ 1917

0.

$110 \quad 15$

0.

11114

0.

$\begin{array}{lll}1 & 12 & 14\end{array}$

$-2.8059 E-04$ 11315

$-3.2832 E-03$ $114 \quad 17$

$-3.7412 E-04$ 11618

11016

0 .

11115

0.

$112 \quad 15$

$0: 1316$

0 .

11418

\section{4}

0.

157

5. $3993 E-03$

1513

0. 519

0. 613

0

1619

0.

1714

0.

1810

$-1.1224 E-03$

1816

0.

1913

0.

1919

0.

$110 \quad 17$

0.1116

0.

11216

0.1317

0.

$114 \quad 19$
1415

0.

158

1514

0.

168

$5.7737 E-0$

1614

0.79

$-1.5144 E-02$

1715

$\begin{array}{lll}0 . & & \\ 1 & 8 & 11\end{array}$

$\begin{array}{lll}0 . & & \\ 1 & 8 & 17\end{array}$

0.

1

0.

$\begin{array}{lll}1 & 10 & 12\end{array}$

$-2.8059 E-04$

11018

0.

0.

$1 \quad 17 \quad 19$
11117

0 .

11217

0.1318

0 .

$\begin{array}{lll}1 & 15 & 17\end{array}$

$-1.5934 E-0$
0

159

-1. $1114 E-02$

1515

0.

169

$2.0760 E-02$

1615

0.

1

0

1

0.

1

0

1

1

0.

11013

0.

11019

0.

11118

0.

1. 1218

0 .

I 1319

0.

11518
$-5.6118 E-04$
0

1417

0.

1510

0.

1516

0

1610

1616

$\begin{array}{lll}1 & 6 & 16 \\ 1 & 7 & 11\end{array}$

1171

$\begin{array}{lll}1 & 7 & 17\end{array}$

$\begin{array}{lll}0 . & & \\ 1 & 83\end{array}$

0.18

0.

1916

0.

$\begin{array}{lll}1 & 10 & 14\end{array}$

0.

11113

$-2.8059 E-04$

11119

0.

$112 \quad 19$

0.

11416

$-1.0997 \mathrm{E}-02$

11519

$-1.7045 E-02-3.1165 E-04-1.8445 E-02$ 